\title{
CATEGORICITY OF AN ABSTRACT ELEMENTARY CLASS IN TWO SUCCESSIVE CARDINALS SH576
}

\author{
SAHARON SHELAh \\ Institute of Mathematics \\ The Hebrew University \\ Jerusalem, Israel \\ Rutgers University \\ Department of Mathematics \\ New Brunswick, NJ USA
}

\begin{abstract}
We investigate categoricity of abstract elementary classes without any remnants of compactness (like non-definability of well ordering, existence of E.M. models, or existence of large cardinals). We prove (assuming a weak version of GCH around $\lambda$ ) that if $\mathfrak{K}$ is categorical in $\lambda, \lambda^{+}, L S(\mathfrak{K}) \leq \lambda$ and $1 \leq I\left(\lambda^{++}, \mathfrak{K}\right)<2^{\lambda^{++}}$ then $\mathfrak{K}$ has a model in $\lambda^{+++}$.
\end{abstract}

Partially supported by the United States-Israel Binational Science Foundation.

I thank Alice Leonhardt for the excellent typing.

Done 10/94, (set theoretic, pseudo descriptive set theory) 22-29/11/94 (essentially $§ 1-\S 2$ ); more $9-14 / 12 / 95$

(fulfilling $\S 1, \S 3$ eliminating use of the maximal model in $\lambda^{+2}$, (new $\S 4$ ) of categoricity in $\lambda^{+2}$ (see $\left.\S 7, \S 8\right)$, more $18-2$ of $4 / 95$ (redo $\left.\S 3, \S 10\right)$.

$\S 10$ written $1 / 2 / 95$

First typed at Rutgers - 95/Feb/10

Latest Revision 98/May/15 


\section{Annotated Content}

[We give three versions of the main theorem in $0.2-0.4$. In $0.5-0.32$ we review the relevant knowledge of abstract elementary classes to help make this paper self-contained. This includes the representation by PC-classes defined by omission of quantifier free types $(0.13,0.14)$; types and stability (based on $\leq_{\mathfrak{K}}$ ); and the equivalence of saturation to model homogeneity $(0.26)$.]

\section{$\S 1 \quad$ Weak Diamond}

WWe mainly present necessary material on the weak diamond, a combinatorial principle whose main variant holds for $\lambda^{+}$if $2^{\lambda}<2^{\lambda^{+}}$.

We state cases provable from ZFC together with suitable cardinal arithmetic assumptions (1.2). We present applications of weak diamond to the number of models of fixed cardinality (notably in 1.10). We deal also with the definable weak diamond, and introduce a smaller ideal of "small" sets: $\operatorname{UDmId}^{\mathscr{F}}(\lambda)(1.14$ - 1.17).]

$\underline{\text { First Attempts }}$

[We define the class $K_{\lambda}^{3}$ of triples $(M, N, a)$ representing types in $\mathscr{S}(M)$ for $M \in K_{\lambda}$, and start to investigate it, dealing with the weak extension property, the extension property, minimality, reduced types (except for minimality, in the first order case, these hold trivially). Our aims are to have the extension property or at least the weak extension property for all triples in $K_{\lambda}^{3}$, and the density of minimal triples. The first property makes the model theory more like the first order case, and the second is connected with categoricity. We start by proving the weak extension property under reasonable assumptions. We prove the density of minimal triples under the strong assumption $K_{\lambda^{+3}}=\emptyset$ and an extra cardinal arithmetic assumption $\left(2^{\lambda^{+}}>\lambda^{++}\right)$. In the end, under the additional assumption $K_{\lambda+3}=0$ we prove that all triples have the extension property and that we have disjoint amalgamation in $K_{\lambda}$. Now the assumption $K_{\lambda+3}=0$ does no harm if we just want to prove Theorem 0.2. The reader willing to accept these assumptions may skip some proofs later. The proof of the extension property makes essential use of categoricity in $\lambda^{+}$.]

\section{$\underline{\text { Non-structure }}$}

[We try to present clearly and in some generality the construction of many models in $\lambda^{++}$based on knowledge of models of size $\lambda^{+}$, using weak diamond on $\lambda^{+}$and on $\lambda^{++}$. This is done by forming a tree $\left\langle\bar{M}^{\eta}: \eta \in^{\lambda^{++}>} 2\right\rangle$ with

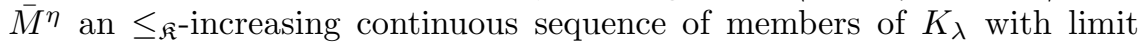
$\bigcup_{i<\lambda^{+}} M_{i}^{\eta}$ increasing with $\eta$ (and an additional restriction). Actually $\lambda^{+}$ can be replaced by a regular uncountable $\lambda^{\prime}$ (so $\left\|M_{i}^{\eta}\right\|=\lambda$ is replaced by $\left.\left.\left\|M_{i}^{\eta}\right\|<\lambda^{\prime}\right)\right]$. 
[We prove that every member of $K_{\lambda}^{3}$ has the extension property, by proving it for minimal triples. We use: if $M_{\ell} \in K_{\lambda}$ and for every minimal $p \in \mathscr{S}\left(M_{0}\right)$ the set $\mathscr{S}_{\geq p}\left(M_{1}\right)$ has cardinality $\leq \lambda^{+}$, then the $M \in K_{\lambda^{+}}$ is saturated for minimal types and hence the number of minimal types in $\mathscr{S}\left(M_{1}\right)$ is $\leq \lambda^{+}$(for $\left.M_{1} \in K_{\lambda}\right)$, which is a step toward stability in $\lambda$.]

Inevitable types and stability in $\lambda$

[We continue to "climb the ladder", using the amount of structure we already have (and sometimes categoricity) to get more. We start by assuming there are minimal types, and show that some minimal types are inevitable, construct $p_{i} \in \mathscr{S}\left(N_{i}\right)$ minimal $\left(i \leq \lambda^{+}\right)$both strictly increasing continuous and with $p_{0}, p_{\delta}$ inevitable, and then as in the proof of the equivalence of saturativity and model homogeneity, we show $N_{\delta}$ is universal over $N_{0}$. We can then deduce stability in $\lambda$, so the model in $\lambda^{+}$is saturated. Then we note that we have disjoint amalgamation in $K_{\lambda}$.]

$\underline{\text { A proof for } \mathfrak{K} \text { categorical in } \lambda^{+2}}$

[We give a shortcut to proving the main theorem by using stronger assumptions. If $I\left(\lambda^{+2}, K\right)=1$ and $I\left(\lambda^{+3}, K\right)=0$ then in some triple $(M, N, a) \in K_{\lambda^{+}}, a$ is essentially algebraic over $M$, i.e. this is a maximal triple. Now first assuming for some pair $M_{0} \leq_{\mathfrak{K}} M_{2}$ in $K_{\lambda}$ we have unique amalgamation for every possible $M_{1}$ with $M_{0} \leq_{\mathfrak{K}} M_{1} \in K_{\lambda}$ (and using stability), we get models in $\lambda^{++}$thus contradicting the existence of maximal triples. We then use the methods of $\S 3$ to prove there are enough cases of unique amalgamation.]

Extensions and Conjugacy

[We investigate types. We prove that in $\mathscr{S}(N), N \in K_{\lambda}$ that reduced implies inevitable, and that non-algebraic extensions preserve the conjugacy classes (so solving the realize/ materialize problem). Hence if $\left\langle N_{i}: i<\alpha\right\rangle$ is $<_{\mathfrak{K}}$-increasing in $K_{\lambda}$ and $\lambda$ divides $\alpha$ then $N_{\alpha}$ is $(\lambda, \operatorname{cf}(\alpha))$-saturated over $N_{0}$.]

Uniqueness of Amalgamation in $\mathfrak{K}_{\lambda}$

[We have by $\S 6$ only $M_{0}^{*}<_{\mathfrak{K}} M_{2}^{*}$ in $\mathfrak{K}_{\lambda}$ such that $M_{0}^{*} \leq_{\mathfrak{K}} M_{1} \Rightarrow M_{0}^{*}, M_{1}, M_{2}^{*}$ has unique (disjoint) amalgamation. Now if we have a $\leq_{\mathfrak{K}}$-increasing continuous sequence $\left\langle N_{i}: i \leq \alpha\right\rangle$ such that $\left(N_{i}, N_{i+1}\right) \cong\left(M_{0}^{*}, M_{2}^{*}\right)$, we can amalgamate $N_{0}, M_{1}, N_{\alpha}$ whenever $N_{0} \leq_{\mathfrak{K}} M_{1}$, step by step. So some uniqueness is preserved and $N_{\alpha}$ can be any $(\lambda, \operatorname{cf}(\alpha))$-saturated model over $N_{0}$. When we require also saturativity of $M_{i}$ and of the resulting model, we get a nonforking relation denoted $\mathrm{NF}_{\lambda, \bar{\delta}}$. We define the general nonforking relation $\mathrm{NF}_{\lambda}$ by closing $\mathrm{NF}_{\lambda, \bar{\delta}}$ downward. So we succeed to define a relation which should behave as a nice nonforking relation. But we have to work to prove that this relation satisfies the expected properties, first for the "saturated" version and then in the general case by a diagram chase.]

$\S 9 \quad$ Nice extensions in $K_{\lambda^{+}}$

[As we have a notion of "nonforking" amalgamation in $K_{\lambda}$, we can use it to build $\leq_{\mathfrak{K}^{-}}$-extensions $M_{1} \in K_{\lambda^{+}}$for any given $M_{0} \in K_{\lambda^{+}}$. This defines 
naturally a two-place relation $\leq_{\lambda^{+}}^{*}$ on $K_{\lambda^{+}}$; "being a nice $\leq_{\mathfrak{K}^{-}}$-submodel". We investigate it and variants. In particular, we prove the existence of disjoint amalgamation.]

$\S 10 \quad$ Non-structure for $<_{\lambda+}^{*}$

[Instead proving that all disjoint amalgamations in $K_{\lambda}$ are nonforking ones, we prove that on $K_{\lambda^{+}}$the relation $<_{\lambda^{+}}^{*}$ is the same as $\leq_{\lambda}^{*}$, which is just as good for our purpose. Toward this we assume a failure and get many pairwise non-isomorphic models in $K_{\lambda^{+2}}$, contradicting an assumption of $0.2(2)$. But once we have that $\leq_{\mathfrak{K}}$ agrees on $K_{\lambda^{+}}$with $\leq_{\lambda^{+}}^{*}$ we have disjoint amalgamation, which suffices for building a model in $K_{\lambda+3}$.] 


\section{$\S 0$ INTRODUCTION}

Makowski [Mw85] is a readable and good exposition concerning categoricity in abstract elementary classes around $\aleph_{1}$.

Our primary concern is:

Problem 0.1. Can we have some (not necessarily much) classification theory for reasonable non-first order classes $\mathfrak{K}$ of models, with no uses of even traces of compactness and only mild set theoretic assumptions?

Let me try to clarify the meaning of Problem 0.1.

What is the meaning of "mild set theoretic assumptions?" We are allowing requirements on cardinal arithmetic like GCH and weaker relatives. Preferably, assumptions like diamonds and squares and even mild large cardinals will not be used (apart from cases provable in ZFC, or in ZFC plus allowable assumptions).

In fact we try to continue [Sh 88], where results about the number of nonisomorphic models in $\aleph_{1}$ and $\aleph_{2}$ of a sentence $\psi \in L_{\omega_{1}, \omega}$ are obtained. Now in [Sh 88] the theorem parallel to the present one is proved assuming $2^{\aleph_{0}}<2^{\aleph_{1}}$, so it is quite natural to use such assumptions here.

What is the meaning of "some classification theory?" While the dream is to have a classification theory as "full" as the one obtained in [Sh:c], we will be glad to have theorems speaking just on having few models in some cardinals or even categoricity and at least one in others. E.g. by [Sh 88] if $\psi \in L_{\omega_{1}, \omega}$ satisfies $1 \leq I\left(\aleph_{1}, \psi\right)<2^{\aleph_{1}}$ (and $\left.2^{\aleph_{0}}<2^{\aleph_{1}}\right)$ then $I\left(\aleph_{2}, \psi\right)>0$. Here $I(\mu, \mathfrak{K})$ is the number of models in $\mathfrak{K}$ of cardinality $\mu$, up to isomorphism.

What are "reasonable non first order classes?" This means we allow classes of "locally finite" or "atomic" structures, or structures "omitting a type", or more generally the class of models of a sentence in $L_{\kappa, \omega}$, (i.e. allowing conjunction $<\kappa$ but quantification only over a finite string) but not one restricting ourselves to e.g. well orderings. In fact, we use "abstract elementary classes" from [Sh 88] (reviewed below).

What is the meaning of "uses traces of compactness?" For non first order classes we cannot use the powerful compactness theorem, but there are many ways to get weak forms of it: one way is using large cardinals (compact cardinals in Makkai Shelah [MaSh 285], or just measurable cardinals as in Kolman Shelah [KlSh 362], or in [Sh 472]). Another way is to use "non-definability of well ordering" which follows from the existence of Ehrenfeucht-Mostowski models, and also from $\psi \in$ $L_{\omega_{1}, \omega}$ having uncountable models (used extensively in [Sh 88]). Our aim is to use none of this and we would like to see if any theory is left.

Above all, we hope the proofs will initiate classification theory in this case, so we hope the flavour will be one of introducing and investigating notions of a model theoretic character. Proofs of, say, a descriptive set theory character, will not satisfy this hope.

It seems to us that this goal is met here. We prove:

Theorem 0.2. $\left(2^{\lambda}<2^{\lambda^{+}}<2^{\lambda^{++}}\right)$. Let $\mathfrak{K}$ be an abstract elementary class. If $\mathfrak{K}$ categorical in $\lambda, \lambda^{+}$and $\lambda^{++}$then $I\left(\lambda^{+3}, \mathfrak{K}\right)>0$.

Here $\lambda^{+3}=\lambda^{+++}$and in general $\aleph_{\alpha}^{+\beta}$ means $\aleph_{\alpha+\beta}$.

Of course, the categoricity in three successive cardinals is a strong assumption. 
Now note that in [Sh 88], the categoricity in $\aleph_{0}$ is gained "freely", so the gap is smaller than seems at first glance. Still it is better to have

Theorem 0.3. $\left(2^{\lambda}<2^{\lambda^{+}}<2^{\lambda^{++}}\right)$. Let $\mathfrak{K}$ be an abstract elementary class.

If $\mathfrak{K}$ is categorical in $\lambda$ and $\lambda^{+}$, and $1 \leq I\left(\lambda^{++}, \mathfrak{K}\right)<2^{\lambda^{++}}$then $I\left(\lambda^{+3}, \mathfrak{K}\right)>0$.

Here we do not fully prove 0.3 but we prove a slightly weaker version:

Theorem 0.4. If $\mathfrak{K}$ is categorical in $\lambda$ and $\lambda^{+}$with $\lambda>\aleph_{0}$ and $1 \leq I\left(\lambda^{++}, \mathfrak{K}\right)<$ $\mu_{\mathrm{wd}}\left(\lambda^{++}\right)$, then $I\left(\lambda^{+3}, \mathfrak{K}\right)>0$.

Note however:

( $\alpha$ ) A silly point; at exactly one point in the proof of 0.4 we assume $\lambda>\aleph_{0}$ (in the proof of 4.6). This is silly as our intent is to prove for general $\lambda$ what we know for $\lambda=\aleph_{0}$ by [Sh 88]; however, there we assume $\mathfrak{K}$ is $\mathrm{PC}_{\aleph_{1}, \aleph_{0}}$, a reasonable assumption, but one which is not assumed here. We shall complete this in [Sh 600, §2], so we do not mention the assumption $\lambda>\aleph_{0}$ in theorems relying on 4.6 .

( $\beta$ ) More seriously, at some point we assume toward a contradiction that $K_{\lambda^{+3}}=$ $\emptyset$. This is fine for proving theorem 0.2 , but is not desirable if we want to develop a classification theory. This will also be dealt with in [Sh 600, §2].

$(\gamma)$ Concerning $\mu_{\mathrm{wd}}\left(\lambda^{+2}\right)$ : in 6.12 we get $I\left(\lambda^{++}, K\right) \geq \mu_{\mathrm{wd}}\left(\lambda^{++}\right)$instead of $I\left(\lambda^{++}, K\right)=2^{\lambda^{++}}$(eliminated in [Sh 600], too). The cardinal $\mu_{\mathrm{wd}}(\chi)$ is defined in 1.1(6). It is almost $2^{\chi}$; i.e. always $2^{\chi}<\mu_{\mathrm{wd}}\left(\chi^{+}\right) \leq 2^{\chi^{+}} \leq$ $\mu_{\mathrm{wd}}\left(\chi^{+}\right)^{\aleph_{0}}$. Already in [Sh 87b] sometimes instead of getting $2^{\chi}$ we get only $\mu_{\mathrm{wd}}(\chi)$ (again see [Sh 600]). If you are satisfied with $I(\chi, K) \geq \mu_{\mathrm{wd}}(\chi)$ in those cases, you can ignore some proofs.

We present below, as background, the following open questions which appeared in [Sh 88], for $\mathfrak{K}$ an abstract elementary class, of course, e.g. the class of models of $\psi \in L_{\lambda^{+}, \omega}$ with the relation $M \leq_{\mathfrak{K}} N$ being $M \prec \mathscr{L} N$ for $\mathscr{L}$ a fragment of $L_{\kappa^{+}, \omega}$ to which $\psi$ belongs. In [Sh 87a], [Sh 87b], [Sh 88] we prove:

$(*)_{3}$ categoricity (of $\left.\psi \in L_{\omega_{1}, \omega}(Q)\right)$ in $\aleph_{1}$ implies the existence of a model of $\psi$ of cardinality $\aleph_{2}$;

$(*)_{4}$ if $n>0,2^{\aleph_{0}}<2^{\aleph_{1}}<\cdots<2^{\aleph_{n}}, \psi \in L_{\omega_{1}, \omega}$ and $1 \leq I\left(\aleph_{\ell}, \psi\right)<\mu_{w d}\left(\aleph_{\ell}\right)$ for $1 \leq \ell \leq n$, then $\psi$ has a model of cardinality $\aleph_{n+1}$.

Now the problems were:

Problem 1) Prove $(*)_{3},(*)_{4}$ in the context of an abstract elementary class $\mathfrak{K}$ which is $P C_{\aleph_{0}}$.

Problem 2) Parallel results in ZFC; e.g. prove $(*)_{3}$ when $n=1,2^{\aleph_{0}}=2^{\aleph_{1}}$.

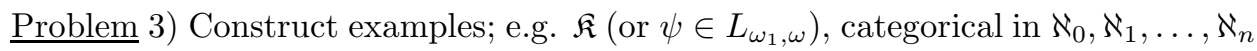
but not in $\aleph_{n+1}$.

Problem 4) If $\mathfrak{K}$ is $P C_{\lambda}$ (abstract elementary class), and is categorical in $\lambda$ and $\lambda^{+}$, does it necessarily have a model in $\lambda^{++}$?

Concerning Problem 3, by Hart Shelah [HaSh 323],2.10(2) +3.8 there is $\psi_{n} \in$ $\mathscr{L}_{\omega_{1}, \omega}$ categorical in $\aleph_{0}, \aleph_{1}, \ldots, \aleph_{k-1}$, but not categorical in $\lambda$ if $2^{\lambda}>2^{\aleph_{k-1}}$. 
The direct motivation for the present work, is that Grossberg asked me (Oct. 94) some questions in this neighborhood, in particular:

Problem 5) Assume $K=\operatorname{Mod}(T)$, (i.e. $K$ is the class of models of $T$ ), $T \subseteq L_{\omega_{1}, \omega},|T|=\lambda, I(\lambda, K)=1$ and $1 \leq I\left(\lambda^{+}, K\right)<2^{\lambda^{+}}$. Does it follow that $I\left(\lambda^{++}, K\right)>0$ ?

We think of this as a test problem and much prefer a model theoretic to a set theoretic solution. This is closely related to Problem 4 above and to [Sh 88, Theorem 3.7] (where we assume categoricity in $\lambda^{+}$, do not require $2^{\lambda}<2^{\lambda^{+}}$but take $\lambda=\aleph_{0}$ or some similar cases) and [Sh 88, Theorem 5.17(4)] (and see [Sh 88, 5.1,4.5] on the assumptions) (there we require $2^{\lambda}<2^{\lambda^{+}}, 1 \leq I\left(\lambda^{+}, K\right)<2^{\lambda^{+}}$and $\lambda=\aleph_{0}$ ).

As said above, we are dealing with a closely related problem. Problem 0.1 was stated a posteriori but is, I think, the real problem.

In a first try we used more set theory, i.e. we used the definitional weak diamond on both $\lambda^{+}$and $\lambda^{++}$(see Definition 1.13) and things like "a nice equivalence relation on $\mathscr{P}(\lambda)$ has either few or many classes" (see $\S 1$ ).

Here we take a model theoretic approach.

We feel that this paper provides a reasonable positive solution to problem 0.1, with a classification theory flavor. We shall continue in [Sh 600] toward a parallel of [Sh 87a], [Sh 87b]. Grossberg and Shelah, in the mid-eighties, started to write a paper [GrSh 266] to prove that: if $\psi \in L_{\lambda^{+}, \omega}$ has models of arbitrarily large cardinality, and is categorical in $\mu^{+n}$ for each $n$ and if $\mu \geq \lambda$ and $2^{\mu^{+n}}<2^{\mu^{+n+1}}$ for $n<\omega$, then $\psi$ is categorical in every $\mu^{\prime} \geq \mu$; this is a weak form of the upward part of Los' conjecture. See Makkai Shelah [MaSh 285] on $T \subseteq \mathscr{L}_{\kappa, \omega}$ where $\kappa$ is a compact cardinal; where we get downward and upward theorems for successor cardinals which are sufficiently bigger than $\kappa+|T|$. On the downward part, see [KlSh 362], [Sh 472] which deals with a downward theorem for successor cardinals which are sufficiently larger than $\kappa+|T|$ when the theory $T$ is in the logic $\mathscr{L}_{\kappa, \omega}$ and $\kappa$ is measurable. See also [Sh 394] which deals with abstract elementary classes with amalgamation, getting similar results with no large cardinals.

Part of $\S 1$ and $\S 3$ have a combinatorial character. Most of the paper forms the content of a course given in Fall '94 (essentially without $\S 3, \S 9, \S 10$ ). The paper is written with an eye to developing the model theory, rather than just proving Theorem 0.2 .

On abstract elementary classes see [Sh 88] and [Sh 300, II,§3]. To make the paper self-contained, we will review some relevant definitions and results. We thank Gregory Cherlin for improving the writing of $\S 1-\S 3$ and for breaking 3.25, 3.26 and 3.27 to three proofs.

This work is continued in [Sh 600]. 


\section{AbstraCt ELEMENTARY CLASSES}

0.5 Conventions. $\mathfrak{K}=\left(K, \leq_{\mathfrak{K}}\right)$, where $K$ is a class of $\tau$-models for a fixed vocabulary $\tau=\tau_{K}=\tau_{\mathfrak{K}}$ and $\leq_{\mathfrak{K}}$ is a two-place relation on the models in $K$. We do not always strictly distinguish between $K$ and $\left(K, \leq_{\mathfrak{K}}\right)$. We shall assume that $K, \leq_{\mathfrak{K}}$ are fixed, and $M \leq_{\mathfrak{K}} N \Rightarrow M, N \in K$; and we assume that the following axioms hold. When we use $<$ in the sense of elementary submodel for first order logic, we write $<\mathscr{L}_{\omega, \omega}$.

\subsection{Definition.}

$A x$ 0: The validity of $M \in K$ or of $N \leq_{\mathfrak{K}} M$ depends on $N$ and $M$ only up to isomorphism - in the second case, isomorphism of the pair.

$A x I:$ If $M \leq_{\mathfrak{K}} N$ then $M \subseteq N$ (i.e. $M$ is a submodel of $N$ ).

$A x I I: \leq_{\mathfrak{K}}$ is transitive and reflexive on $K$.

Ax III: If $\lambda$ is a regular cardinal, $M_{i}(i<\lambda)$ is $\leq_{\mathfrak{K}}$-increasing (i.e. $i<j<\lambda$ implies $M_{i} \leq_{\mathfrak{K}} M_{j}$ ) and continuous (i.e. for limit ordinals $\delta<\lambda$ we have

$\left.M_{\delta}=\bigcup_{i<\delta} M_{i}\right) \underline{\text { then }} M_{0} \leq_{\mathfrak{K}} \bigcup_{i<\lambda} M_{i}$.

$A x I V$ : If $\lambda$ is a regular cardinal, $M_{i}(i<\lambda)$ is $\leq_{\mathfrak{K}}$-increasing continuous, $M_{i} \leq_{\mathfrak{K}} N$ then $\bigcup_{i<\lambda} M_{i} \leq_{\mathfrak{K}} N$.

$A x V$ : If $M_{0} \subseteq M_{1}$ and $M_{\ell} \leq_{\mathfrak{K}} N$ for $\ell=0,1$, then $M_{0} \leq_{\mathfrak{K}} M_{1}$.

$A x V I$ : There is a cardinal $\lambda$ such that: if $A \subseteq N$ and $|A| \leq \lambda$ then for some $M \leq_{\mathfrak{K}} N$ we have $A \subseteq|M| \leq \lambda$. We define the Löwenheim-Skolem number $L S(\mathfrak{K})$ as the least such $\lambda$ with $\lambda \geq|\tau|$.

Notation: $K_{\lambda}=\{M \in K:\|M\|=\lambda\}$ and $K_{<\lambda}=\bigcup_{\mu<\lambda} K_{\mu}$. $\mathscr{L}_{\omega, \omega}$ is first order logic.

A theory in $\mathscr{L}(\tau)$ is a set of sentences from $\mathscr{L}(\tau)$.

0.7 Definition. The embedding $f: N \rightarrow M$ is a $\mathfrak{K}$-embedding or a $\leq_{\mathfrak{K}}$-embedding if its range is the universe of a model $N^{\prime} \leq_{\mathfrak{K}} M$, (so $f: N \rightarrow N^{\prime}$ is an isomorphism (onto)).

Very central in [Sh 88], but peripheral here is:

0.8 Definition. 1) For a logic $\mathscr{L}$ and vocabulary $\tau, \mathscr{L}(\tau)$ is the set of $\mathscr{L}$-formulas in this vocabulary.

2 ) Let $T_{1}$ be a theory in $\mathscr{L}_{\omega, \omega}\left(\tau_{1}\right), \tau \subseteq \tau_{1}$ vocabularies, $\Gamma$ a set of types in $\mathscr{L}_{\omega, \omega}\left(\tau_{1}\right)$; (i.e. for some $m$, a set of formulas $\left.\varphi\left(x_{0}, \ldots, x_{m-1}\right) \in \mathscr{L}_{\omega, \omega}\left(\tau_{1}\right)\right)$.

3) $E C\left(T_{1}, \Gamma\right)=\left\{M: M\right.$ a $\tau_{1}$-model of $T_{1}$ which omits every $\left.p \in \Gamma\right\}$.

4) $P C_{\tau}\left(T_{1}, \Gamma\right)=P C\left(T_{1}, \Gamma, \tau\right)=\left\{M: M\right.$ is a $\tau$-reduct of some $\left.M_{1} \in E C\left(T_{1}, \Gamma\right)\right\}$.

5) We say that $\mathfrak{K}$ is $P C_{\lambda}^{\mu}$ if for some $T_{1}, T_{2}, \Gamma_{1}, \Gamma_{2}$ and $\tau_{1}$ and $\tau_{2}$ we have: $T_{\ell}$ is a first order theory in the vocabulary $\tau_{\ell}, \Gamma_{\ell}$ is a set of types and $K=P C\left(T_{1}, \Gamma_{1}, \tau_{\mathfrak{K}}\right)$ and $\left\{(M, N): M \leq_{\mathfrak{K}} N, M, N \in K\right\}=P C\left(T_{2}, \Gamma_{2}, \tau^{\prime}\right)$ where 
$\tau^{\prime}=\tau_{1} \cup\{P\}(P$ a new one place predicate $),\left|T_{\ell}\right| \leq \lambda,\left|\Gamma_{\ell}\right| \leq \mu$ for $\ell=1,2$. If $\mu=\lambda$, we may omit it.

0.9 Example. If $\tau_{1}=\tau, T_{1}, \Gamma$ are as above, and $\left(K, \leq_{\mathfrak{K}}\right)$ are defined by $K=: E C\left(T_{1}, \Gamma\right), \leq_{\mathfrak{K}}=: \prec \mathscr{L}_{\omega, \omega}$, then the pair satisfies the Axioms from 1.2 and $L S(\mathfrak{K}) \leq\left|T_{1}\right|+\aleph_{0}$.

0.10 Example. $(V=L)$.

Let $\operatorname{cf}(\lambda) \geq \aleph_{1}, n<\omega$ then for some $\psi \in L_{\lambda^{+}, \omega}$ we have: $\psi$ has no model of cardinality $\lambda^{+(n+1)}$, and is categorical in $\lambda^{+n}$ (i.e. has one and only one model up to isomorphism).

Let $M^{*}=\left(L_{\lambda^{+n}}, \in, i\right)_{i \leq \lambda}$ and let $\psi$ be

$$
\begin{aligned}
& \bigwedge\left\{\varphi: \varphi \text { is a first order sentence which } M^{*} \text { satisfies }\right\} \\
& \wedge(\forall x)\left(x \in \lambda \equiv \bigvee_{i<\lambda} x=i\right) .
\end{aligned}
$$

0.11 Lemma. Let I be a directed set (i.e. partially ordered by $\leq$, such that any two elements have a common upper bound).

1) If $M_{t}$ is defined for $t \in I$, and $t \leq s \in I$ implies $M_{t} \leq_{\mathfrak{K}} M_{s}$ then for every $t \in I$ we have $M_{t} \leq_{\mathfrak{K}} \bigcup_{s \in I} M_{s}$.

2) If in addition $t \in I$ implies $M_{t} \leq_{\mathfrak{K}} N \underline{\text { then }} \bigcup_{s \in I} M_{s} \leq_{\mathfrak{K}} N$.

Proof. By induction on $|I|$ (simultaneously for 1 ) and 2)), or see $[$ Sh $88,1.6]$. $\square_{0.11}$

\subsection{Lemma.}

1) Let $\tau_{1}=\tau \cup\left\{F_{i}^{n}: i<L S(\mathfrak{K})\right.$ and $\left.n<\omega\right\}, F_{i}^{n}$ an n-place function symbol (assuming, of course, $F_{i}^{n} \notin \tau$ ). If $M_{1}$ is an expansion of $M$ to a $\tau_{1}$-model and $\bar{a} \in$ ${ }^{n}|M|$ for some $n$, let $M_{\bar{a}}$ be the submodel of $M$ with universe $\left\{F_{i}^{n}(\bar{a}): i<L S(k)\right\}$. Every model $M$ from $K$ can be expanded to a $\tau_{1}$-model $M_{1}$ such that:

(A) $M_{\bar{a}} \leq_{\mathfrak{K}} M$ for any $\bar{a} \in{ }^{n}|M|$;

(B) if $\bar{a} \in{ }^{n}|M|$ then $\left\|M_{\bar{a}}\right\| \leq L S(\mathfrak{K})$;

(C) if $\bar{b}$ is a subsequence of $\bar{a}$ (even up to rearrangement), then $M_{\bar{b}} \leq_{\mathfrak{K}} M_{\bar{a}}$;

(D) for every $N_{1} \subseteq M_{1}$ we have $N_{1} \uparrow \tau \leq_{\mathfrak{K}} M$.

2) If $N \leq_{\mathfrak{K}} M$ is given, then we can choose the expansion $M_{1}$ so that clauses (A) - (D) hold and

(E) $N=N_{1} \uparrow \tau$ for some $N_{1} \subseteq M_{1}$. 
Proof. We define by induction on $n$, the values of $F_{i}^{n}(\bar{a})$ for every $i<L S(\mathfrak{K})$, $\bar{a} \in{ }^{n}|M|$ such that $\bar{a} \subseteq N \Rightarrow M_{\bar{a}} \subseteq N$ when we are proving (2). By $A x V I$ there is an $M_{\bar{a}} \leq_{\mathfrak{K}} M,\left\|M_{\bar{a}}\right\| \leq L S(\mathfrak{K})$ such that $\left|M_{\bar{a}}\right|$ includes

$$
\bigcup\left\{M_{\bar{b}}: \bar{b} \text { a subsequence of } \bar{a} \text { of length }<n\right\} \cup \bar{a}
$$

and $M_{\bar{a}}$ does not depend on the order of $\bar{a}$. Let $\left|M_{\bar{a}}\right|=\left\{c_{i}: i<i_{0} \leq L S(\mathfrak{K})\right\}$, and define $F_{i}^{n}(\bar{a})=c_{i}$ for $i<i_{0}$ and $F_{i}^{n}(\bar{a})=c_{0}$ for $i_{0} \leq i<L S(\mathfrak{K})$ (so we can demand " $F_{i}^{n}$ is symmetric").

Clearly our conditions are satisfied: if $\bar{b}$ is a subsequence of $\bar{a}$ then $M_{\bar{b}} \leq_{\mathfrak{K}} M_{\bar{a}}$ by $A x V$.

0.13 Lemma. 1) There is a set $\Gamma$ of types in $\mathscr{L}_{\omega, \omega}\left(\tau_{1}\right)$ (where $\tau_{1}$ is as in Lemma $0.12)$ such that $K=P C(\emptyset, \Gamma, \tau)$, so it is a $P C_{L S(\mathfrak{K})}^{2^{L S(\mathfrak{K})}}$-class.

The types above consist of quantifier-free formulas.

2) Moreover, if $N_{1} \subseteq M_{1} \in E C(\emptyset, \Gamma)$, and $N, M$ are the $\tau$-reducts of $N_{1}, M_{1}$ respectively then $N \leq_{\mathfrak{K}} M$. Also, if $N \leq_{\mathfrak{K}} M$ then there is an $\tau_{1}$-expansion $M_{1}$ of $M$ and a submodel $N_{1}$ of $M_{1}$ such that $M_{1} \in E C(\emptyset, \Gamma)$ and $N_{1}\lceil\tau=N$.

3) Also $\left\{(M, N): N \leq_{\mathfrak{K}} M\right\}$ is a $P C_{L S(\mathfrak{K})}^{\left(2^{L(\mathfrak{K})}\right)}$-class, hence $\mathfrak{K}$ is as well.

Proof. 1) Let $\Gamma_{n}$ be the set of complete quantifier free $n$-types $p\left(x_{0}, \ldots, x_{n-1}\right)$ in $\mathscr{L}_{\omega, \omega}\left(\tau_{1}\right)$ such that: if $M_{1}$ is an $L_{1}$-model, $\bar{a}$ realizes $p$ in $M_{1}$ and $M$ is the $L$-reduct of $M_{1}$, then $M_{\bar{b}} \leq_{\mathfrak{K}} M_{\bar{a}}$ for any subsequence of $\bar{b}$ of $\bar{a}$. Where $M_{\bar{c}}\left(\right.$ for $\left.\bar{c} \in{ }^{m}\left|M_{1}\right|\right)$ is the submodel of $M$ whose universe is $\left\{F_{i}^{m}(\bar{c}): i<L S(\mathfrak{K})\right\}$ (and there are such submodels).

Let $\Gamma$ be the set of $p$ which are complete quantifier free $n$-types for some $n<\omega$ in $\mathscr{L}_{\omega, \omega}\left(\tau_{1}\right)$ and which do not belong to $\Gamma_{n}$. So if $M^{1}$ is in $P C\left(\emptyset, \Gamma, \tau_{1}\right)$ then by 0.11 we have $M_{1}\lceil\tau \in K$ hence $P C(\emptyset, \Gamma, L) \subseteq K$ and by $0.12(1)$ we have $K \subseteq P C(\emptyset, \Gamma, L)$.

2) The first phrase is proven as in (1). For the second phrase, use 0.12(2).

3) Follows from (2).

0.14 Conclusion. There is $\tau_{1}$ with $\tau \subseteq \tau_{1}$ and $\left|\tau_{1}\right| \leq L S(\mathfrak{K})$ such that: for any $M \in K$ and any $\tau_{1}$-expansion $M_{1}$ of $M$ which is in $E C(\emptyset, \Gamma)$,

$$
\begin{gathered}
N_{1} \subseteq M_{1} \Rightarrow N_{1}\left\lceil\tau \leq_{\mathfrak{K}} M\right. \\
N_{1} \subseteq N_{2} \subseteq M_{1} \Rightarrow N_{1}\left\lceil\tau \leq_{\mathfrak{K}} N_{2}\lceil\tau .\right.
\end{gathered}
$$

0.15 Conclusion. If for every $\alpha<\left(2^{L S(\mathfrak{K}}\right)^{+}, K$ has a model of cardinality $\geq \beth_{\alpha}$ then $K$ has a model in every cardinality $\geq L S(\mathfrak{K})$.

Proof. Use 0.13 and the value of the Hanf number for: models of a first order theory omitting a given set of types, for languages of cardinality $L S(K)$ (see [Sh:c, VII,, 5$])$. 
0.16 Definition. For $\lambda$ regular $>L S(\mathfrak{K})$ and $N \in \mathfrak{K}_{\lambda}$ we say $\bar{N}=\left\langle N_{\alpha}: \alpha<\lambda\right\rangle$ is a representation of $N$ if $\bar{N}$ is $\leq_{\mathfrak{K}}$-increasing continuous, $\left\|N_{\alpha}\right\|<\lambda$ and $N=\bigcup_{\alpha<\lambda} N_{\alpha}$. If $\lambda=\mu^{+}$then if not said otherwise, we require $\left\|N_{\alpha}\right\|=\mu$.

How will we define types, and in particular, the set $\mathscr{S}(M)$ of complete types over $M$, when no formulas are present? If we have a "monster model" $\mathfrak{C}$ we can use automorphisms; but any such "monster" is far down the road. So we will "chase diagrams" in $K_{\lambda}$ (being careful, not to use excessivley large models). This gives us a relation of "having the same type" we call $E_{\mu}^{a t}$, but this relation in general is not transitive (if we do not have amalgamation in $K_{\lambda}$ ). So $E_{\mu}$ will be defined as the transitive closure of $E_{\mu}^{a t}$.

0.17 Definition. 1) The two-place relation $E_{M}^{\mathrm{at}}$ is defined on triples $(M, N, a)$ with $M$ fixed, $M \leq_{\mathfrak{K}} N \in K_{\|M\|}$, and $a \in N$ by:

$$
\begin{aligned}
& \left(M, N_{1}, a_{1}\right) E_{M}^{\mathrm{at}}\left(M, N_{2}, a_{2}\right) \underline{\text { iff }} \text { there is } N \in K_{\mu} \text { and } \leq_{\mathfrak{K}} \text {-embeddings } \\
& \qquad \begin{array}{l}
f_{\ell}: N_{\ell} \rightarrow N \text { for } \ell=1,2 \text { such that: } \\
f_{1} \uparrow M=\operatorname{id}_{M}=f_{2}\left\lceil M \text { and } f_{1}\left(a_{1}\right)=f_{2}\left(a_{2}\right) .\right.
\end{array}
\end{aligned}
$$

Let $E_{M}$ be the transitive closure of $E_{M}^{\text {at }}$.

2) For $\mu \geq L S(\mathfrak{K})$ and $M \in K_{\mu}$ we define $\mathscr{S}(M)$ as $\left\{\operatorname{tp}(a, M, N): M \leq_{\mathfrak{K}} N \in\right.$ $K_{\mu}$ and $\left.a \in N\right\}$ where $\operatorname{tp}(a, M, N)=(M, N, a) / E_{M}$.

3) We say " $a$ realizes $p$ in $N$ " if $a \in N, p \in \mathscr{S}(M)$ and for some $N^{\prime} \in K_{\mu}$ we have $M \leq_{\mathfrak{K}} N^{\prime} \leq_{\mathfrak{K}} N, a \in N^{\prime}$, and $p=\operatorname{tp}\left(a, M, N^{\prime}\right)$.

4) We say " $a_{2}$ strongly realizes $\left(M, N^{1}, a^{1}\right) / E_{M}^{\text {at }}$ in $N$ " if for some $N^{2}, a^{2}$ we have $M \leq_{\mathfrak{K}} N^{2} \leq_{\mathfrak{K}} N, a_{2} \in N^{2}$, and $\left(M, N^{1}, a^{1}\right) E_{M}^{a t}\left(M, N^{2}, a^{2}\right)$.

5) We say $M_{0} \in \mathfrak{K}$ is an amalgamation base if letting $\lambda=\left\|M_{0}\right\|$ we have: for every

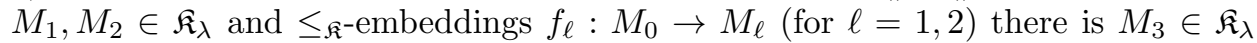
and $\leq_{\mathfrak{K}^{-}}$-embeddings $g_{\ell}: M_{\ell} \rightarrow M_{3}$ (for $\left.\ell=1,2\right)$ such that $g_{1} \circ f_{1}=g_{2} \circ f_{2}$.

6) We say $\mathfrak{K}$ is stable in $\lambda$ if $L S(\mathfrak{K}) \leq \lambda$ and for all $M \in K_{\lambda}$ we have $|\mathscr{S}(M)| \leq \lambda$.

0.18 Observation. If $M$ is an amalgamation base then $E_{M}=E_{M}^{\text {at }}$, and we have:

" $a$ strongly realizes $(M, N, b) / E_{M}$ in $N$ " iff " $a$ realizes $(M, N, b) / E_{M}$.

\subsection{Definition.}

1) $\mathfrak{K}$ has the $\lambda$-amalgamation property or has amalgamation in $\lambda$, if every $M_{0} \in \mathfrak{K}_{\lambda}$ is an amalgamation base (see $0.17(5)$ above).

2) $N$ is universal over $M$ if: $M \leq_{\mathfrak{K}} N$ and if $M \leq_{\mathfrak{K}} N^{\prime} \in K_{\leq\|N\|}$ then $N^{\prime}$ can be $\leq_{\mathfrak{K}}$-embedded into $N$ over $M$.

3) $\mathfrak{K}$ has universal extensions in $\lambda$ if for every $M \in K_{\lambda}$ there is $N$ such that:

(a) $M \leq_{\mathfrak{K}} N \in K_{\lambda}$

(b) $N$ is universal over $M$. 
4) $N_{1}, N_{2}$ have a joint embedding if for some $N \in K$ there are $\leq_{\mathfrak{K}}$-embeddings $h_{\ell}$ of $N_{\ell}$ into $N$ for $\ell=1,2$. Let $\mathrm{JEP}_{\mu}$ (JEP) means this holds for $N_{1}, N_{2}$ in $K_{\mu}$ (in $K)$.

5) $\mathfrak{K}_{\lambda}$ has unique amalgamation (or $\mathfrak{K}$ has unique amalgamation in $\lambda$ ) when: if $M_{i}^{\ell} \in K_{\lambda}$ for $\ell<2, i<4$, and for $i=1,2$ we have $h_{i, 0}^{\ell}$ is a $\leq_{\mathfrak{K}^{-}}$-embedding of $M_{0}^{\ell}$ into $M_{i}^{\ell}, h_{3, i}^{\ell}$ is a $\leq_{\mathfrak{K}^{-}}$-embedding of $M_{i}^{\ell}$ into $M_{3}^{\ell}, h_{3,1}^{\ell} \circ h_{1,0}^{\ell}=h_{3,2}^{\ell} \circ h_{2,0}^{\ell}$ and $\operatorname{Rang}\left(h_{3,1}^{\ell}\right) \cap \operatorname{Rang}\left(h_{3,2}^{\ell}\right)=\operatorname{Rang}\left(h_{3,1}^{\ell} \circ h_{1,0}^{\ell}\right)$ and for $i<3, f_{i}$ is an isomorphism from $M_{i}^{0}$ onto $M_{i}^{1}, f_{0} \subseteq f_{1}, f_{0} \subseteq f_{2}$ then for some $N \in K_{\lambda}$ there are $\mathfrak{K}$-embedding $h_{\ell}: M_{3}^{\ell} \rightarrow N$ such that $h_{0} \circ h_{3, i}^{0}=h_{1} \circ h_{3, i}^{1}$, for $i=1,2$.

6) Let $p_{\ell} \in \mathscr{S}\left(M_{\ell}\right)$ for $\ell=0,1$. We say $p_{0} \leq p_{1}$ if $M_{0} \leq_{\mathfrak{K}} M_{1}$ and for some $N$ and $a$ we have $M_{1} \leq_{\mathfrak{K}} N \in K_{\left\|M_{1}\right\|+L S(\mathfrak{K})}, a \in N$ and $\operatorname{tp}\left(a, M_{\ell}, N\right)=p_{\ell}$ for $\ell=0,1$. We also write $p_{0}=p_{1} \uparrow M_{0}$ ( $p_{0}$ is unique knowing $M_{0}, M_{1}, p_{1}$ hence $p_{1} \uparrow M_{0}$ is well defined).

\subsection{Claim.}

1) If $\mathfrak{K}$ is categorical in $\lambda$ and $L S(\mathfrak{K}) \leq \lambda$ then: there is a model in $K_{\lambda^{+}}$iff for some (equivalently, every) model $M \in K_{\lambda}$ there is $N$ such that $M<_{\mathfrak{K}} N \in K_{\lambda}$ iff for some (equivalently every) $M \in K_{\lambda}$ there is $N$ such that $M<_{\mathfrak{K}} N \in K_{\lambda^{+}}$.

2) If $\mathfrak{K}$ has amalgamation in $\lambda, L S(\mathfrak{K}) \leq \lambda$, and $M_{0} \leq_{\mathfrak{K}} M_{1}$ are in $K_{\lambda}$ with $M_{0} \leq_{\mathfrak{K}}$ $N_{0} \in K_{\lambda^{+}}$then we can find $h$ and $N_{1}$ such that $N_{0} \leq_{\mathfrak{K}} N_{1} \in K_{\lambda^{+}}$and $h$ is a $\leq_{\mathfrak{K}}$-embedding of $M_{1}$ into $N_{1}$ extending $i d_{M_{0}}$. We can allow $N_{0} \in K_{\mu}$ with $\mu>\lambda$ if $\mathfrak{K}$ has amalgamation in every $\lambda^{\prime} \in[\lambda, \mu)$.

3) Assume $\mathfrak{K}$ has amalgamation in $\lambda$ and $L S(\mathfrak{K}) \leq \lambda$. If $M_{0} \leq M_{1}$ are from $K_{\lambda}$ and $p_{0} \in \mathscr{S}\left(M_{0}\right)$ then we can find an extension $p_{1} \in \mathscr{S}\left(M_{1}\right)$ of $p_{0}$.

Proof. 1) As then we can choose by induction on $i<\lambda^{+}$models

$M_{i} \in K_{\lambda}, \leq_{\mathfrak{K}^{-}}$-increasing continuous, $M_{i} \neq M_{i+1}$, for $i=0$ use $K_{\lambda^{+}} \neq \emptyset$, for

$i$ limit take union, for $i=j+1$ use the previous sentence; so

$M_{\lambda^{+}}=\cup\left\{M_{i}: i<\lambda^{+}\right\} \in K_{\lambda^{+}}$as required.

2), 3) Left to the reader.

0.21 Remark. We can here add the content of $6.5,6.7,6.12$.

0.22 Definition. 1) For $\lambda>L S(\mathfrak{K})$ we say " $N \in \mathfrak{K}$ is $\lambda$-saturated" if for every $M \leq_{\mathfrak{K}} N$ of cardinality $<\lambda$, if $M \leq_{\mathfrak{K}} N^{\prime} \in K_{<\lambda}$ and $a^{\prime} \in N^{\prime}$ then some $a \in N$ strongly realizes $\left(M, N^{\prime}, a^{\prime}\right) / E_{M}^{\text {at }}$ (in the interesting cases this suffices).

2) We say " $N \in \mathfrak{K}$ is $\lambda$-saturated above $\mu$ (or is $\lambda$-saturated $\geq \mu$ )" if above we restrict ourselves to $M$ of cardinality $\geq \mu$.

(Cf. $(\lambda, \kappa)$-saturated in Definition 0.28, 0.29 below).

0.23 Fact. 1) If $\mu+L S(\mathfrak{K})<\lambda, \lambda$ is regular, $\mathfrak{K}$ has the amalgamation property in every $\mu^{\prime} \in[\mu, \lambda)$, and for all $M \in K_{[\mu, \lambda)}$ we have $|\mathscr{S}(M)| \leq \lambda$ and $\lambda=\operatorname{cf}(\lambda)$, then there is some $M \in K_{\lambda}$ saturated above $\mu$.

2) Assume $\lambda>\mu \geq L S(\mathfrak{K})$ and $N \in K$ and $\mathfrak{K}$ has amalgamation in $K_{\mu}$, for every $\mu_{1} \in[\mu, \lambda)$. Then: $N$ is $\lambda$-saturated above $\mu$ iff for every $M \leq_{\mathfrak{K}} N$ of cardinality $<\lambda$, every $p \in \mathscr{S}(M)$ is realized in $N$ (i.e. for some $a \in N$ we have $\operatorname{tp}(a, M, N)=p)$. 
3) If $L S(\mathfrak{K}) \leq \mu_{0} \leq \mu_{0}^{\prime} \leq \mu^{\prime} \leq \mu$ and $M$ is $\mu$-saturated above $\mu_{0}$, then it is $\mu^{\prime}$ saturated above $\mu_{0}^{\prime}$. If $L S(\mathfrak{K}) \leq \mu_{0}<\mu$ then: $M$ is $\mu$-saturated above $\mu_{0}$ iff for every $\lambda \in\left[\mu_{0}, \mu\right), M$ is $\lambda^{+}$-saturated above $\lambda$.

0.24 Definition. The type $p \in \mathscr{S}(M)$ is local when: if for any directed partial order $I$ and models $M_{t} \leq_{\mathfrak{K}} M$ for $t \in I$ with $I \models t \leq s \Rightarrow M_{t} \leq_{\mathfrak{K}} M_{s}$ and $M=\bigcup_{t \in I} M_{t}$, and any $p^{\prime} \in \mathscr{S}(M)$ with $\left(p\left\lceil M_{t}=p^{\prime} \uparrow M_{t}\right)\right.$ for all $t \in I, p=p^{\prime}$. We say $M$ is local if every $p \in \mathscr{S}(M)$ is, and $\mathscr{K}$ is local if every $M \in \mathscr{K}$ is. We can add "above $\mu$ " as in Definition 0.22(2).

0.25 Definition. 1) $I(\lambda, K)=I(\lambda, \mathfrak{K})$ is the number of $M \in K_{\lambda}$ up to isomorphism. 2) $\mathfrak{K}$ (or $K$ ) is categorical in $\lambda$ if $I(\lambda, K)=1$.

3) $I E(\lambda, \mathfrak{K})=\sup \left\{\left|K^{\prime}\right|: K^{\prime} \subseteq K_{\lambda}\right.$ and for $M \neq N$ in $K_{\lambda}^{\prime}, M$ is not $\leq_{\mathfrak{K}}$-embeddable into $N\}$. If we write $\operatorname{IE}(\lambda, \bar{K}) \geq \mu$, we mean that for some $K^{\prime} \subseteq K_{\lambda}$ as above, $\left|K^{\prime}\right| \geq \mu$, and similarly for $=\mu$. If there is a problem with attainment of the supremum we shall say explicitly.

0.26 The Model-homogeneity $=$ Saturativity Lemma. Let $\lambda>\mu \geq L S(\mathfrak{K})$ and $M \in K$.

1) $M$ is $\lambda$-saturated above $\mu$ iff $M$ is $\left(\mathscr{D}_{\mathfrak{K}}, \lambda\right)$-homogeneous above $\mu$, which means: for every $N_{1} \leq_{\mathfrak{K}} N_{2} \in K$ such that $\mu \leq\left\|N_{1}\right\| \leq\left\|N_{2}\right\|<\lambda$ and $N_{1} \leq_{\mathfrak{K}} M$ there is $a \leq_{\mathfrak{K}}$-embedding $f$ of $N_{2}$ into $M$ over $N_{1}$.

2) If $M_{1}, M_{2} \in K_{\lambda}$ are $\lambda$-saturated above $\mu<\lambda$ and for some $N_{1} \leq_{\mathfrak{K}} M_{1}, N_{2} \leq_{\mathfrak{K}}$ $M_{2}$, both of cardinality $\in[\mu, \lambda)$, we have $N_{1} \cong N_{2}$ then $M_{1} \cong M_{2}$; in fact, any isomorphism $f$ from $N_{1}$ onto $N_{2}$ can be extended to an isomorphism from $M_{1}$ onto $\mathrm{M}_{2}$.

3) If in (2) we demand only " $M_{2}$ is $\lambda$-saturated" and $M_{1} \in K_{\leq \lambda}$ then $f$ can be extended to a $\leq_{\mathfrak{K}}$-embedding from $M_{1}$ into $M_{2}$.

4) In part (2) instead of $N_{1} \cong N_{2}$ it suffices to assume that $N_{1}$ and $N_{2}$ can be $\leq_{\mathfrak{K}}$-embedded into some $N \in K$, which holds if $\mathfrak{K}$ has the JEP.

Proof. 1) The "if" direction is easy as $\lambda>L S(\mathfrak{K})$. Let us prove the other direction, so without loss of generality $\left\|N_{1}\right\|=\mu$. $\left\|N_{1}\right\|$.

By $0.19(6)$ without loss of generality $\lambda$ is regular, moreover $N_{2}$ has cardinality

Let $\left|N_{2}\right|=\left\{a_{i}: i<\kappa\right\}$, and we know $\kappa=\left\|N_{1}\right\|=\left\|N_{2}\right\|<\lambda$. We define by induction on $i \leq \kappa, N_{1}^{i}, N_{2}^{i}, f_{i}$ such that:

(a) $N_{1}^{i} \leq_{\mathfrak{K}} N_{2}^{i}$ and $\left\|N_{2}^{i}\right\|<\lambda$

(b) $N_{1}^{i}$ is $\leq_{\mathfrak{K}}$-increasing continuous in $i$

(c) $N_{2}^{i}$ is $\leq_{\mathfrak{K}}$-increasing continuous in $i$

(d) $f_{i}$ is a $\leq_{\mathfrak{K}^{-}}$-embedding of $N_{1}^{i}$ into $M$

(e) $f_{i}$ is increasing continuous in $i$

(f) $a_{i} \in N_{1}^{i+1}$

(g) $N_{1}^{0}=N_{1}, N_{2}^{0}=N_{2}, f_{0}=i d_{N_{1}}$

(h) $N_{1}^{i}$ and $N_{2}^{i}$ has cardinality $\kappa$. 
For $i=0$, clause $(g)$ gives the definition. For $i$ limit let:

$$
\begin{aligned}
& N_{1}^{i}=\bigcup_{j<i} N_{1}^{j} \text { and } \\
& N_{2}^{i}=\bigcup_{j<i} N_{2}^{j} \text { and } \\
& f_{i}=\bigcup_{j<i} f_{j} .
\end{aligned}
$$

Now (a)-(f) continues to hold by continuity (and $\left\|N_{2}^{i}\right\|<\lambda$ as $\lambda$ is regular).

For $i$ successor we use our assumption; more elaborately, let $M_{1}^{i-1} \leq_{\mathfrak{K}} M$ be $f_{i-1}\left(N_{1}^{i-1}\right)$ and $M_{2}^{i-1}, g_{i-1}$ be such that $g_{i-1}$ is an isomorphism from $N_{2}^{i-1}$ onto $M_{2}^{i-1}$ extending $f_{i-1}$, so $M_{1}^{i-1} \leq_{\mathfrak{K}} M_{2}^{i-1}$ (but without loss of generality $\left.M_{2}^{i-1} \cap M=M_{1}^{i-1}\right)$. Now apply the saturation assumption with $M, M_{1}^{i-1}$, $\operatorname{tp}\left(g_{i-1}\left(a_{i_{1}}\right), M_{1}^{i-1}, M_{2}^{i-1}\right)$ here standing for $N, M, p$ there (note: $a_{i-1} \in N_{2}=N_{2}^{0} \subseteq$ $N_{2}^{i-1}$ and $\mu>\left\|N_{2}^{i-1}\right\|=\left\|M_{2}^{i-1}\right\|$ and $\left\|M_{1}^{i-1}\right\|=\left\|N_{1}^{i-1}\right\| \geq\left\|N_{1}^{0}\right\|=\left\|N_{1}\right\| \geq \mu_{0}$ so the requirements and also the cardinality in Definition 0.22 holds). So there is $b \in M$ such that $\operatorname{tp}\left(b, M_{1}^{i-1}, M\right)=\operatorname{tp}\left(g_{i-1}\left(a_{i-1}\right), M_{1}^{i-1}, M_{2}^{i-1}\right)$. Moreover (if $\mathfrak{K}$ has amalgamation in $\mu$ the proof is slightly shorter) remembering the second sentence in $0.22(1)$ which speaks about "strongly realizes" there is $b \in M$ such that $b$ strongly realizes $\left(M_{1}^{i-1}, M_{2}^{i-1}, g_{i-1}\left(a_{i-1}\right)\right) / E_{M_{1}^{i-1}}^{\text {at }}$ in $M$. This means (see Definition $0.17(3))$ that for some $M_{1}^{i, *}$ we have $M_{1}^{i-1} \leq_{\mathfrak{K}} M_{1}^{i, *} \leq_{\mathfrak{K}} M$ and $\left(M_{1}^{i-1}, M_{2}^{i-1}, g_{i-1}\left(a_{i-1}\right)\right) E_{M_{1}^{i-1}}^{\mathrm{at}}\left(M_{1}^{i-1}, M_{1}^{i, *} b\right)$. This means (see Definition 0.17(1) that $M^{\prime}$ too has cardinality $\kappa$ and there is $M_{i}^{2, *} \in K_{\mu}$ such that $M_{1}^{i-1} \leq_{\mathfrak{K}} M_{2}^{i, *}$ and

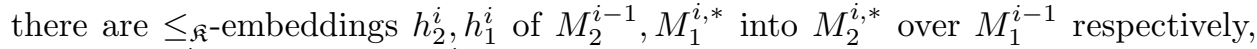
such that $h_{2}^{i}\left(g_{i-1}\left(a_{i-1}\right)\right)=h_{1}^{i}(b)$.

Now changing names, without loss of generality $h_{2}^{i}$ is the identity.

Let $N_{2}^{i}, h_{i}$ be such that $N_{2}^{i-1}<_{\mathfrak{K}} N_{2}^{i}, h_{i}$ an isomorphism from $N_{2}^{i}$ onto $M_{2}^{i, *}$ extending $g_{i-1}$. Let $N_{1}^{i}=h_{i}^{-1} \circ h_{1}^{i}\left(M_{1}^{i, *}\right), f_{i}=\left(h_{1}^{i}\right)^{-1} \circ\left(h_{i}\left\lceil N_{1}^{i}\right)\right.$.

We have carried the induction. Now $f_{\kappa}$ is a $\leq_{\mathfrak{K}^{-}}$embedding of $N_{1}^{\kappa}$ into $M$ over $N_{1}$, but $\left|N_{2}\right|=\left\{a_{i}: i<\kappa\right\} \subseteq N_{1}^{\kappa}$, so $f_{\kappa} \uparrow N_{2}: N_{2} \rightarrow M$ is as required.

$2), 3)$ By the hence and forth argument (or see [Sh 300, II,§3] $=[$ Sh:e, II,$\S 3]$ ).

4) Easy, too.

0.27 Remark. Note that by $0.26(2)$ if $M$ is $\mu$-saturated above $\mu_{0}$ and $\mathfrak{K}$ has the $\mathrm{JEP}_{\mu_{0}}$ then $\mathfrak{K}$ has $\lambda$-amalgamation for each $\lambda \in\left[\mu_{0}, \mu\right)$.

0.28 Definition. Fix $\lambda \geq \kappa$ with $\kappa$ regular.

1) We say that $N_{1} \in K_{\lambda}$ is $(\lambda, \kappa)$-saturated over $N_{0}$ or that $\left(N_{1}, c\right)_{c \in N_{0}}$ is $(\lambda, \kappa)$ saturated (and $\lambda$-saturated of cofinality $\kappa$ means $(\lambda, \kappa)$-saturated) if:

there is a sequence $\left\langle M_{i}: i<\kappa\right\rangle$ which is $\leq_{\mathfrak{K}^{-}}$-increasing continuous with $M_{0}=$ $N, M_{\kappa}=N_{1}$ and $M_{i+1} \in K_{\lambda}$ universal over $M_{i}$ (see 0.19(2)).

2) If we omit $\kappa$, we mean $\kappa=\operatorname{cf}(\lambda) ;(\lambda, \alpha)$-saturated means $(\lambda, \operatorname{cf}(\alpha))$-saturated; and $N_{1}$ is $(\lambda, 1)$-saturated over $N_{0}$ means just $N_{0} \leq_{\mathfrak{K}} N_{1}$ are in $K_{\lambda}$. 
0.29 Claim. Fix $\lambda \geq \kappa$ with $\kappa$ regular.

1) If $N_{1}$ is $(\lambda, \kappa)$-saturated over $N$, then $N_{1}$ is unique over $N$.

2) If $K_{\lambda} \neq \emptyset, \lambda \geq \kappa=c f(\kappa)$ and over every $M \in K_{\lambda}$ there is $N$ with $M \leq_{\mathfrak{K}} N \in K_{\lambda}$ universal over $M$, then for every $N \in K_{\lambda}$ there is $N_{1} \in K_{\lambda}$ which is $(\lambda, \kappa)$-saturated over $N$.

3) If $\mathfrak{K}_{\lambda}$ has amalgamation and $\mathfrak{K}$ is stable in $\lambda$ (i.e. $M \in K_{\lambda} \Rightarrow|\mathscr{S}(M)| \leq \lambda$ ) then every $M \in K_{\lambda}$ has a universal extension (so part (2)'s conclusion holds).

Proof. See [Sh 300, Ch.II] or check.

We do not need at present but recall from [Sh 88]:

0.30 Claim. There is $\tau^{\prime} \supseteq \tau \cup\left\{P_{0}, P_{1}, P_{2}, c\right\}$ of cardinality $\leq L S(\mathfrak{K})$ with $c$ an individual constant, with $P_{\ell}$ unary predicates, and a set $\Gamma$ of quantifier free types such that:

(a) if $M^{\prime} \in P C_{\tau^{\prime}}(\emptyset, \Gamma)$ and $M_{\ell}=\left(M^{\prime} \uparrow \tau\right) \uparrow P_{\ell}^{M^{\prime}}$ for $\ell=0,1,2$, then

$M_{\ell} \in K, M_{0} \leq_{\mathfrak{K}} M_{1}, M_{0} \leq_{\mathfrak{K}} M_{2}, c^{M^{\prime}} \in M_{2}$, and

$N^{\prime} \subseteq M^{\prime} \Rightarrow\left(N^{\prime}\lceil\tau) \uparrow P_{\ell}^{N^{\prime}} \leq_{\mathfrak{K}} M_{\ell}\right.$, and there is no $b \in M_{1}$ satisfying:

$\otimes$ for every $\bar{a} \in \omega>\left(P_{0}^{M^{\prime}}\right)$, letting $N_{\bar{a}}$ be the $\tau^{\prime}$-submodel of $M^{\prime}$ generated by $\bar{a}$ and $M_{\bar{a}}^{\ell}=M_{\ell} \uparrow\left(M_{\ell} \cap N_{\bar{a}}\right)$, we have $M_{\bar{a}}^{\ell} \leq_{\mathfrak{K}} M_{\ell}$, and $b$ strongly realizes $\operatorname{tp}\left(c^{M^{\prime}}, M_{\bar{a}}^{0}, M_{\bar{a}}^{2}\right)$ in $M_{\bar{a}}^{1}$

(b) if $M_{\ell}^{*}, c$ are as in (a) and $M_{0}=M_{1} \cap M_{2}$. then for some $M^{\prime}$ we have clause (a).

Proof. Should be clear; see [Sh 88].

\subsection{Remark.}

1) Claim 0.30 enables us to translate results of the form: two cardinal with omitting types theorem in $\lambda_{2}$ implies the existence of one in $\lambda_{1}$, provided that types are local in the sense that $p \in \mathscr{S}(M)$ is determined by $\left\langle p\left\lceil N: N \leq_{\mathfrak{K}} M,\|N\| \leq \lambda\right\rangle\right.$.

2) This enables us to prove implications between cases of $\lambda$-categoricity, if we have a nice enough theory of types as in [Sh:c, VIII, $\S 4$ ]; if we have in $\lambda_{2}$ a saturated model, categoricity in $\lambda_{1}$ implies categoricity in $\lambda_{2}$. Also (if we know a little more) categoricity in $\lambda_{2}$ is equivalent to the non-existence of a non-saturated model in $\lambda_{2}$.

0.32 Claim. 1) Assume $M_{n} \leq_{\mathfrak{K}} M_{n+1}, M_{n} \in K_{\lambda}$, $\mathfrak{K}$ has amalgamation in $\lambda$. If $p_{n} \in \mathscr{S}\left(\mathscr{M}_{n}\right), p_{n} \leq p_{n+1}$ (i.e. $p_{n}=p_{n+1}\left\lceil M_{n}\right.$, see 0.19(6)), then there is $p \in \mathscr{S}\left(\bigcup_{n<\omega} M_{n}\right)$ such that $n<\omega \Rightarrow p_{n} \leq p$.

2) If $\left\langle M_{i}: i \leq \delta\right\rangle$ is $\leq_{\mathfrak{K}}$-increasing continuous, $p_{i} \in \mathscr{S}\left(M_{i}\right),\left(j<i \Rightarrow p_{j} \leq p_{i}\right)$, $p_{i}=t p\left(a_{i}, M_{i}, N_{i}\right)$ and $h_{i, j}$ is a $\leq_{\mathfrak{K}}$-embedding of $N_{j}$ into $N_{i}($ for $j<i<\delta)$ such that $h_{i, j}\left\lceil M_{j}=i d_{M_{j}}, h_{i, j}\left(a_{j}\right)=a_{i}\right.$, then there is $p_{\delta} \in \mathscr{S}\left(M_{\delta}\right), i \leq \delta \Rightarrow p_{i} \leq p_{\delta}$. 3) If $\mathfrak{K}$ has amalgamation in $\lambda$ and is stable in $\lambda$ (i.e. $M \in K_{\lambda} \Rightarrow|\mathscr{S}(M)| \leq \lambda$ ), then 
(a) every $M \in K_{\lambda}$ has a universal extension;

(b) for every $M \in K_{\lambda}$ and regular $\theta \leq \lambda$ there is $N \in K_{\lambda}$ which is $(\lambda, \theta)$ saturated over $M$. 


\section{$\S 1$ Weak Diamond}

1.1 Definition. Fix $\lambda$ regular and uncountable.

1)

$\operatorname{WDmTId}(\lambda, S, \bar{\chi})=\left\{A: A \subseteq \prod_{\alpha \in S} \chi_{\alpha}\right.$, and for some function $F$ with domain

$\bigcup_{\alpha<\lambda}^{\alpha}\left(2^{<\lambda}\right)$ mapping ${ }^{\alpha}\left(2^{<\lambda}\right)$ into $\chi_{\alpha}$

for every $\eta \in A$, for some $f \in{ }^{\lambda}\left(2^{<\lambda}\right)$ the set

$\{\delta \in S: \eta(\delta)=F(f\lceil\delta)\}$ is not stationary $\}$.

(Note: WDmTId stand for weak diamond target ideal) ${ }^{1}$.

Here we can replace $2^{<\lambda}$ by any set of this cardinality, and so we can replace $f \in{ }^{\lambda}\left(2^{<\lambda}\right)$ by $f_{1}, \ldots, f_{n} \in{ }^{\lambda}\left(2^{<\lambda}\right)$ with $F$ being $n$-place.

2) $\operatorname{cov}_{\mathrm{wdmt}}(\lambda, S, \bar{\chi})=\operatorname{Min}\left\{|\mathscr{P}|: \mathscr{P} \subseteq \operatorname{WDmTId}(\lambda, S)\right.$ and $\left.S_{2} \subseteq \bigcup_{A \in \mathscr{P}} A\right\}$

3)

$\operatorname{WDmTId}_{<\mu}(\lambda, S, \bar{\chi})=\left\{A:\right.$ for some $i^{*}<\mu$ and $A_{i} \in \operatorname{WDmTId}(\lambda, S)$ for $i<i^{*}$ we have $\left.A \subseteq \bigcup_{i<i^{*}} A_{i}\right\}$

4)

$$
\mathrm{WDmId}_{<\mu}(\lambda, \bar{\chi})=\left\{S \subseteq \lambda: \operatorname{cov}_{\mathrm{wdmt}}(\lambda, S)<\mu\right\}
$$

5) Instead of " $<\mu^{+}$" we write $\mu$, if we omit $\mu$ we mean $\left(2^{<\lambda}\right)$. If $\bar{\chi}$ is constantly 2 we may omit it, if $\chi_{\alpha}=2^{|\alpha|}$ we may write pow instead of $\bar{\chi}$.

$$
\text { Let } \mu_{\mathrm{wd}}(\lambda, \bar{\chi})=\operatorname{cov}_{\mathrm{wdmt}}(\lambda, \lambda, \bar{\chi}) \text {. }
$$

7) We say that the weak diamond holds on $\lambda$ if $\lambda \notin \operatorname{WDmId}(\lambda)$.

By [DvSh 65], [Sh:b, XIV,1.18(2),1.8] (presented better in [Sh:f, AP,§1], note: 1.2(4) below rely on [Sh 460]) we have:

\footnotetext{
${ }^{1}$ in $[\mathrm{Sh}: \mathrm{b}, \mathrm{AP}, \S 1]$, [Sh:f, AP,, 1$]$ we express $\operatorname{cov}_{\mathrm{Wdmt}}(\lambda, S)>\mu^{*}$ by allowing $f(0) \in \mu^{*}<\mu$
} 


\subsection{Theorem.}

1) If $\lambda=\aleph_{1}, 2^{\aleph_{0}}<2^{\aleph_{1}}, \mu \leq\left(2^{\aleph_{0}}\right)$ or even $2^{\theta}=2^{<\lambda}<2^{\lambda}, \mu=\left(2^{\theta}\right)^{+}$, or just: for some $\theta, 2^{\theta}=2^{<\lambda}<2^{\lambda}, \mu \leq 2^{\lambda}$, and $\chi^{<\lambda}<\mu$ for $\chi<\mu$, then $\lambda \notin W D m I d_{<\mu}(\lambda)$. If in addition $(*)_{<\mu, \lambda}$ below holds, then $\lambda \notin W D m I d_{<\mu}(\lambda$, pow $)$, where:

$(*)_{\mu, \lambda}$ there are no $A_{i} \in[\mu]^{\lambda^{+}}$for $i<2^{\lambda}$ such that $i \neq j \Rightarrow\left|A_{i} \cap A_{j}\right|<\aleph_{0}$ and $(*)_{<\mu, \lambda}$ means $(*)_{\chi, \lambda}$ holds for $\chi<\mu$.

2) If $\mu \leq \lambda^{+}$or $c f\left(\left[\mu_{1}\right]^{\leq \lambda}, \subseteq\right)<\mu$ for $\mu_{1}<\mu$ or $\mu=\aleph_{0} \underline{\text { then }} \operatorname{WDmId}_{<\mu}(\lambda, \bar{\chi})$ is a normal ideal on $\lambda$. If this ideal is not trivial, then $\lambda=\operatorname{cf}(\lambda)>\aleph_{0}, 2^{<\lambda}<2^{\lambda}$.

3) A sufficient condition for $(*)_{<\mu, \lambda}$ is:

(a) $\mu \leq 2^{\lambda} \&(\forall \alpha<\mu)\left(|\alpha|^{\aleph_{0}}<2^{\lambda}\right)$.

4) Another sufficient condition for $(*)_{<\mu, \lambda}$ is:

(b) $\mu \leq 2^{\lambda} \& \lambda \geq \beth_{\omega}$.

1.3 Remark. 1) So if $\operatorname{cf}\left(2^{\lambda}\right)<\mu$ (which holds if $2^{\lambda}$ is singular and $\mu=2^{\lambda}$ ) then $(*)_{<\mu, \lambda}$ implies that there is $A \subseteq{ }^{\lambda} 2,|A|<2^{\lambda}, A \notin \operatorname{WDmTId}(\lambda)$.

2) Some related definitions appear in 1.13; we use them below (mainly $\operatorname{DfWD}_{<\mu}(\lambda)$ ), but as in a first reading it is recommended to ignore them, the definition is given later.

3) We did not look again at the case $(\forall \sigma<\lambda)\left(2^{\sigma}<2^{<\lambda}<2^{\lambda}\right)$.

As in [Sh 88, 3.5], ([Sh 87a, 2.7], [Sh 87b, 6.3]):

1.4 Claim. Assume $\lambda \notin W D m I d_{<\mu}(\lambda)$ or at least $D f W D_{<\mu}(\lambda)$ (where $\lambda=c f(\lambda)>\aleph_{0}$ ) and $\mathfrak{K}$ is an abstract elementary class.

1) Assume $\mathfrak{K}$ is categorical in $\chi, \lambda=\chi^{+}$, and $\mathfrak{K}$ has a model in $\lambda$ (if $L S(\mathfrak{K}) \leq \chi$ this is equivalent to: the model $M \in K_{\chi}$ is not $\leq_{\mathfrak{K}}$-maximal). Assume further $\mathfrak{K}$ does not have the $\lambda$-amalgamation property in $\chi$. Then for any $M_{i} \in \mathfrak{K}_{\lambda}$ for $i<i^{*}<\mu$, there is $N \in \mathfrak{K}_{\lambda}$ not $\leq_{\mathfrak{K}}$-embeddable into any $M_{i}$ (and the assumptions of part (2) below holds).

2) Assume $M_{\eta} \in K_{<\lambda}$ for $\eta \in{ }^{\lambda>} 2, M_{\eta}=\bigcup_{\alpha<\ell g(\eta)} M_{\eta \uparrow(\alpha+1)}, \nu \triangleleft \eta \Rightarrow M_{\nu} \leq_{\mathfrak{K}} M_{\eta}$, and $M_{\eta^{\wedge}\langle 0\rangle}, M_{\eta^{\wedge}\langle 1\rangle}$ cannot be amalgamated over $M_{\eta}$ (hence $\left.M_{\eta} \neq M_{\eta^{\wedge}\langle\ell\rangle}\right)$. Set $M_{\eta}=: \bigcup_{\alpha<\lambda} M_{\eta\lceil\alpha}$ for $\eta \in{ }^{\lambda} 2$. Clearly $M_{\eta}$ belongs to $K_{\lambda}$. For the $D f W D_{<\mu}(\lambda)$ version assume also

(*) $\left\langle M_{\eta}: \eta \in^{\lambda>} 2\right\rangle$ is definable (even just by $\mathscr{L}_{\lambda, \lambda}$ ) in $\mathfrak{B}=\left(\mathscr{H}(\chi), \in,<_{\chi}^{*}, \mathfrak{K}_{<\chi}, \lambda, \mu\right)$.

$\underline{\text { Then }}$ for any $N_{i} \in \mathfrak{K}_{\lambda}$ for $i<i^{*}<\mu$, there is $\eta \in{ }^{\lambda} 2$ such that: $M_{\eta}$ is not $\leq_{\mathfrak{K}}$-embeddable into any $N_{i}$.

3) In part (2), if $L S(\mathfrak{K}) \leq \lambda$ we can allow $N_{i} \in K_{\kappa_{i}}$ if $\sum_{i<i^{*}} c f\left(\left[\kappa_{i}\right]^{\lambda}, \subseteq\right)<\mu$.

4) If we use $D f W D_{<\mu}(\lambda)$ : assume only $\lambda \notin W D m I d_{<\mu}(\lambda, \bar{\chi})$. Part (2) holds if $M_{\eta}$ 
is defined for $\eta \in \bigcup_{\alpha<\lambda} \prod_{i<\alpha} \chi_{i}$, and $\varepsilon<\zeta<\chi_{i}, \eta \in \prod_{j<i} \chi_{j} \Rightarrow M_{\eta^{\wedge}\langle\varepsilon\rangle}, M_{\eta^{\wedge}\langle\zeta\rangle}$ cannot be amalgamated over $M_{\eta}$. The assumption of Part (4) holds (hence the conclusion of Part (2)) holds if we assume that for $M \in K_{\chi}, i<\lambda$ there are $\chi_{i} \leq_{\mathfrak{K}}$-extensions of $M$ in $K_{\lambda}$, which pairwise cannot be amalgamated over $M$.

Proof. 1) It is straightforward to choose $M_{\eta} \in K_{\chi}$ for $\eta \in{ }^{\alpha} 2$ by induction on $\alpha$, as required in part (2). Then use part (2) to get the desired conclusion.

2) Without loss of generality the universe of $N_{i}$ is $\lambda$ and the universe of $M_{\eta}$ is an ordinal $\gamma_{\eta}$ such that $\eta \in{ }^{\lambda>} 2 \Rightarrow \gamma_{\eta}<\lambda$ and $\eta \in{ }^{\lambda} 2 \Rightarrow \gamma_{\eta}=\lambda$. The reader can ignore the "DfWD ${ }_{<\mu}(\lambda)$ " version (ignoring the $h_{\eta}$ 's,g) if he likes. For $\alpha<\lambda$ and $\eta \in{ }^{\alpha} 2$ let the function $h_{\eta}$ be $h_{\eta}(i)=M_{\eta \uparrow(i+1)}$ for $i<\ell g(\eta)$. Let $\left\langle M_{\eta}: \eta \in{ }^{\lambda>} 2\right\rangle$ be the $<_{\chi}^{*}$-first such object. For each $i<i^{*}$ we define $A_{i} \subseteq{ }^{\lambda} 2$ by $A_{i}=\left\{\eta \in{ }^{\lambda} 2\right.$ : $M_{\eta}$ can be $\leq_{\mathfrak{K}^{-}}$-embedded into $\left.N_{i}\right\}$.

For $\eta \in A_{i}$ choose $f_{\eta}: M_{\eta} \rightarrow N_{i}$, a $\leq_{\mathfrak{K}^{-}}$embedding, hence $f_{\eta} \in{ }^{\lambda} \lambda$. We also define a function $F_{i}$ from $\bigcup_{\alpha<\lambda}\left({ }^{\alpha} 2 \times{ }^{\alpha} \lambda\right)$ to $\{0,1\}$ by:

$$
\begin{aligned}
F_{i}(\eta, f) \text { is : } 0 \quad \text { if } f \text { is a } \leq \leq_{\mathfrak{K}} \text {-embedding of } M_{\eta} \text { into } N_{i} \\
\text { with range } \subseteq \ell g(\eta) \text { which can be extended to a } \\
\quad \leq_{\mathfrak{K}} \text {-embedding of } M_{\eta^{\wedge}\langle 0\rangle} \text { into } N_{i}
\end{aligned}
$$

\section{$F_{i}(\eta, f)$ is 1 otherwise.}

Now for any $\eta \in A_{i}$, the set

$$
E=\left\{\delta<\lambda: \gamma_{\eta \uparrow \delta}=\delta \text { and } f_{\eta}\lceil\delta \text { is a function from } \delta \text { to } \delta\}\right.
$$

is a club of $\lambda$. For every $\delta \in E$ clearly $F\left(\eta\left\lceil\delta, f_{\eta}\lceil\delta)=\eta(\delta)\right.\right.$ (as $M_{\eta^{\wedge}\langle 0\rangle}, M_{\eta^{\wedge}\langle 1\rangle}$ cannot be amalgamated over $M_{\eta}$ ).

Hence (for the "Def" version see Definition 1.13(2) using 1.14(1), 1.14(3)) we have $A_{i} \in \operatorname{WDmTId}^{\operatorname{Def}}(\lambda)$. As $i^{*}<\mu$ clearly $\bigcup_{i<i^{*}} A_{i} \in \operatorname{WDmId}_{<\mu}^{\text {Def }}(\lambda)$ and hence by assumption of the claim ${ }^{\lambda} 2 \neq \bigcup_{i} A_{i}$. Take $\eta \in{ }^{\lambda} 2 \backslash \bigcup_{i<i^{*}} A_{i}$. Then $M_{\eta}$ is as required.

3) Without loss of generality the universe of $N_{i}$ is $\kappa_{i}$. Let $\mathscr{P}_{i} \subseteq\left[\kappa_{i}\right]^{\lambda}$ be a set of minimal cardinality such that $(\forall B)\left[B \subseteq \kappa_{i} \quad \&|B| \leq \lambda \rightarrow\left(\exists B^{\prime} \in \mathscr{P}_{i}\right)\left(B \subseteq B^{\prime}\right)\right]$. As $L S(\mathfrak{K}) \leq \lambda$ we can find for each $A \in \mathscr{P}_{i}$, a model $N_{A}^{i} \leq_{\mathfrak{K}} N_{i}$ of cardinality $\lambda^{+}$ whose universe includes $A$. Now apply part (2) to $\left\{N_{A}^{i}: i<i^{*}\right.$ and $\left.A \in \mathscr{P}_{i}\right\}$.

4) Same proof.

We give three variants of the preceding: 
1.5 Claim. 1) Assume

$(*)_{1} \quad \lambda=c f(\lambda)>\aleph_{0}$

(a) $M_{\eta}$ is a $\tau$-model of cardinality $<\lambda$ for $\eta \in^{\lambda>2}$ and

(b) for each $\eta \in{ }^{\lambda} 2,\left\langle M_{\eta \uparrow \alpha}: \alpha<\lambda\right\rangle$ is $\subseteq$-increasing continuous with union, called $M_{\eta} \in K$, of cardinality $\lambda$

(c) if $\eta \in{ }^{\lambda>} 2, \eta \triangleleft \rho_{\ell} \in{ }^{\lambda} 2$ for $\ell=1,2$ then $M_{\rho_{1}}, M_{\rho_{2}}$ are not isomorphic over $M_{\eta}$

$(*)_{2} \quad \lambda \notin W D m I d_{<\mu}(\lambda)$

$\underline{\text { Then }} I(\lambda, K) \geq \mu$, and in fact we can find $X \subseteq{ }^{\lambda} 2$ of cardinality $\geq \mu$ such that $\left\{M_{\rho}: \rho \in X\right\}$ are pairwise non-isomorphic.

2) Assume

$(*)_{1}^{d}$ like $(*)_{1}$ in part (1) but in addition

(d) $\left\langle M_{\eta}: \eta \in^{\lambda>2}\right\rangle$ is definable in $\mathfrak{B}=\mathfrak{B}_{\chi}$

$(*)_{2}^{d} \lambda \notin D f W D_{<\mu}(\lambda)$.

Then the conclusion of part (1) holds.

3) The parallel of 1.4(4) holds.

Proof. 1) Let $\left\{N_{i}: i<i^{*}\right\}$ be a maximal subset of $\left\{M_{\rho}: \rho \in{ }^{\lambda} 2\right\}$ consisting of pairwise non-isomorphic models, and use the proof of 1.4(2) with $f_{\eta}: N_{i} \simeq M_{\eta}$. 2), 3) Left to the reader.

\author{
1.5
}

1.6 Claim. 1) Assume

$(*)_{1} M_{\eta} \in K_{<\lambda}$ for $\eta \in{ }^{\lambda>} 2,\left\langle M_{\eta\lceil\alpha}: \alpha \leq \ell g(\eta)\right\rangle$ is $\leq_{\mathfrak{K}}$-increasing continuous, and $M_{\eta^{\wedge}\langle 1\rangle}$ cannot be $\leq_{\mathfrak{K}}$-embedded into $M_{\nu}$ over $M_{\eta}$ when $\eta^{\wedge}\langle 0\rangle \unlhd \nu \in{ }^{\lambda>} 2$

$(*)_{2} \lambda=c f(\lambda)>\aleph_{0}, \lambda \notin W D m I d_{<\mu}(\lambda)$, and $\lambda$ is a successor cardinal, or at least there is no $\lambda$-saturated normal ideal on $\lambda$, or at least $W D m I d(\lambda)$ is not $\lambda$-saturated (which holds if for some $\theta<\lambda,\{\delta<\lambda: c f(\delta)=\theta\} \notin$ $\operatorname{WDmId}(\lambda))$.

Then there is $A \subseteq{ }^{\lambda} 2,|A|=2^{\lambda}$ such that: if $\eta_{1} \neq \eta_{2}$ are in $A$ then (taking $M_{\eta}=$ $\left.\bigcup_{\alpha<\lambda} M_{\eta\lceil\alpha}\right)$

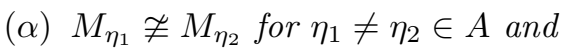

( $\beta$ ) if $\left(2^{\chi}\right)^{+}<2^{\lambda}$ for $\chi<\lambda \underline{\text { then }}$ we can also achieve: $M_{\eta_{1}}$ cannot be $\leq_{\mathfrak{K}^{-}}$ embedded into $M_{\eta_{2}}$.

2) Under the assumptions of 1.4(1) we can find $\left\langle M_{\eta}: \eta \in^{\lambda>} 2\right\rangle$ as in the assumption of 1.4(2).

3) Under the assumption of 1.4(2) the assumption of 1.6(1) holds. 
4) Under the assumption of 1.6(1) we have $I(\lambda, \mathfrak{K})=2^{\lambda}$ and if $\left(2^{\chi}\right)^{+}<2^{\lambda}$ then $\operatorname{IE}(\lambda, \mathfrak{K})=2^{\lambda}$.

5) The parallel of 1.4(4) holds.

Proof of 1.6. 1) The proof of [Sh 88, 3.5] works (see the implications preceding it). More elaborately, we divide the proof into cases according to the answer to the following:

Question: Is there $\eta^{*} \in{ }^{\lambda>} 2$ such that for every $\nu$ satisfying $\eta^{*} \unlhd \nu \in{ }^{\lambda>} 2$ there are $\rho_{0}, \rho_{1} \in{ }^{\lambda}>2$ such that: $\nu \triangleleft \rho_{0}, \nu \unlhd \rho_{1}$, and $M_{\rho_{0}}, M_{\rho_{1}}$ cannot be amalgamated over $M_{\eta^{*}}$ ?

We can find a function $h:{ }^{\lambda>} 2 \rightarrow^{\lambda>} 2$, such that:

(a) the function $h$ is one-to-one, preserving $\triangleleft$ and $(h(\nu))^{\wedge}\langle\ell\rangle \unlhd h\left(\nu^{\wedge}\langle\ell\rangle\right)$

$(b)_{\text {yes }}$ when the answer to the question is yes, it is exemplified by $\eta^{*}=h(\langle\rangle)$ and $M_{h\left(\nu^{\wedge}\langle 0\rangle\right)}, M_{h\left(\nu^{\wedge}\langle 1\rangle\right)}$ cannot be amalgamated over $M_{\eta^{*}}$ (for every $\left.\nu \in{ }^{\lambda>} 2\right)$

$(b)_{\text {no }}$ when the answer to the question above is no, $h(\langle\rangle)=\langle\rangle$ and if $\nu^{\wedge}\langle 0\rangle \unlhd$ $\rho_{0}, \nu^{\wedge}\langle 1\rangle \triangleleft \rho_{1}$ then $M_{h\left(\rho_{0}\right)}, M_{h\left(\rho_{1}\right)}$ can be amalgamated over $M_{h(\nu)}$.

Without loss of generality $h$ is the identity, by renaming (and we can preserve $(*)_{1}^{d}$ of 1.5(2) in the relevant case). Also clearly $M_{\eta^{\wedge}\langle\ell\rangle} \neq M_{\eta}$ (by the non-amalgamation assumption).

$\underline{\text { Case } 1}$ The answer is yes. We do not use the non $\lambda$-saturation of $\operatorname{WDmId}(\lambda)$ in this case.

For any $\eta \in{ }^{\lambda} 2$ and $\leq_{\mathfrak{K}}$-embedding $g$ of $M_{\langle\rangle}$into $M_{\eta}=: \bigcup_{\alpha<\lambda} M_{\eta\lceil\alpha}$, let

$$
\begin{gathered}
A_{\eta, g}=:\left\{\nu \in{ }^{\lambda} 2: \text { there is a } \leq_{\mathfrak{K}} \text {-embedding of } M_{\nu} \text { into } M_{\eta} \text { extending } g\right\} \\
A_{\eta}=:\left\{\nu \in{ }^{\lambda} 2: \text { there is a } \leq_{\mathfrak{K}} \text {-embedding of } M_{\nu} \text { into } M_{\eta}\right\} .
\end{gathered}
$$

So: $\left|A_{\eta, g}\right| \leq 1$ and $\eta \in A_{\eta}$ (as if $\nu_{1}, \nu_{2} \in A_{\eta, g}$ are distinct then for some ordinal $\alpha<\lambda$ and $\nu \in{ }^{\alpha} 2$ we have $\nu=: \nu_{0}\left\lceil\alpha=\nu_{1}\left\lceil\alpha, \nu_{0}(\alpha) \neq \nu_{1}(\alpha)\right.\right.$ and use the choice of $\left.h\left(\nu^{\wedge}\langle\ell\rangle\right)\right)$.

Since $A_{\eta}=\cup\left\{A_{\nu, g}: g\right.$ is a $\leq_{\mathfrak{K}}$-embedding of $M_{\langle\rangle}$into $\left.M_{\eta}\right\}$, we have $\left|A_{\eta}\right| \leq 2^{<\lambda}$. Hence we can choose by induction on $\zeta<2^{\lambda}, \eta_{\zeta} \in{ }^{\lambda} 2 \backslash \bigcup_{\xi<\zeta} A_{\eta_{\xi}}$ (existing by cardinality considerations as $\left.2^{<\lambda}<\lambda\right)$. Then $\xi<\zeta \Rightarrow M_{\eta_{\xi}} \nRightarrow M_{\eta_{\zeta}}$ so we have proved clause $(\alpha)$.

If $\left(2^{<\lambda}\right)^{+}<2^{\lambda}$, then use the Hajnal free subset theorem ([Ha61]) to choose distinct $\left\{\eta_{\zeta}: \zeta<2^{\lambda}\right\} \subseteq{ }^{\lambda} 2$ such that $\zeta \neq \xi \Rightarrow \eta_{\zeta} \notin A_{\eta_{\xi}}$, so we have proved clause $(\beta)$, too.

Case 2: The answer is no.

Again, without loss of generality $M_{\eta}$ has as universe the ordinal $\gamma_{\eta}$.

Let $\left\langle S_{i}: i<\lambda\right\rangle$ be a partition of $\lambda$ to sets, none of which is in $\operatorname{WDmId}(\lambda)$. For each $i$ we define a function $F_{i}$ as follows: 


$$
\begin{aligned}
& \text { if } \delta \in S_{i}, \eta, \nu \in{ }^{\delta} 2, \gamma_{\eta}=\gamma_{\nu}=\delta \text {, and } f: \delta \rightarrow \delta \text { then } \\
& F_{i}(\eta, \nu, f)=0 \text { if } f \text { can be extended to an embedding of } \\
& M_{\nu^{\wedge}\langle 0\rangle} \text { into some } M_{\rho} \text { with } \eta^{\wedge}\langle 0\rangle \unlhd \rho \text {. } \\
& F_{i}(\eta, \nu, f)=1 \text { otherwise. }
\end{aligned}
$$

So as $S_{i} \notin \operatorname{WDmId}(\lambda)$, for some $\eta_{i}^{*} \in{ }^{\lambda} 2$ we have:

(*) for every $\eta \in{ }^{\lambda} 2, \nu \in{ }^{\lambda} 2$ and $f \in{ }^{\lambda} \lambda$ the following set of ordinals $i<\lambda$ is stationary:

$$
\left\{\delta \in S_{i}: F_{i}\left(\eta\left\lceil\delta, \nu \uparrow \delta, f\lceil\delta)=\eta_{i}^{*}(\delta)\right\}\right.\right.
$$

Now for any $X \subseteq \lambda$ let $\eta_{X}, \rho_{X} \in{ }^{\lambda} 2$ be defined by:

$$
\begin{aligned}
& \text { if } \alpha \in S_{i} \text { then } i \in X \Rightarrow \eta_{X}(\alpha)=1-\eta_{i}^{*}(\alpha), i \notin X \Rightarrow \eta_{X}(\alpha)=0 \text { and } \\
& \rho_{X}=\eta_{\{2 i: i \in X\} \cup\{2 i+1: i \notin X\}} .
\end{aligned}
$$

Now we show

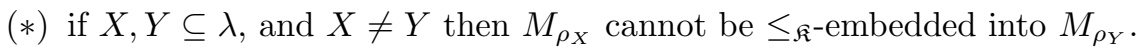

Clearly $(*)$ will suffice for finishing the proof.

Assume toward a contradiction that $f$ is a $\leq_{\mathfrak{K}^{-}}$-embedding of $M_{\rho_{X}}$ into $M_{\rho_{Y}}$; as $X \neq Y$ there is $i$ such that $i \in X \Leftrightarrow i \notin Y$ so there is $j \in\{2 i, 2 i+1\}$ such that $\rho_{X}\left\lceil S_{j}=\left\langle 1-\eta_{j}^{*}(\alpha): \alpha \in S_{j}\right\rangle\right.$ and $\rho_{Y}\left\lceil S_{j}\right.$ is identically zero. Clearly $E=\{\delta: f$ maps $\delta$ into $\delta\}$ is a club of $\lambda$ and hence $S_{j} \cap E \neq \emptyset$.

So if $\delta \in S_{j} \cap E$ then $f\left\lceil M_{\left(\rho_{Y} \uparrow(\delta+1)\right)}=f\left\lceil M_{\left(\rho_{Y}\lceil\delta)^{\wedge}\langle 0\rangle\right.}\right.\right.$ extend $f\left\lceil M_{\rho_{Y}\lceil\delta}\right.$ and is a $\leq_{\mathfrak{K}^{-}}$-embedding of it into some $M_{\left(\rho_{X}\lceil\alpha)\right.}, \alpha<\lambda$ large enough.

Now by the choice of $h$ we get

$$
\delta \in S_{j} \cap E \Rightarrow F\left(\rho _ { X } \left\lceil\delta, \rho_{Y}\left\lceil\delta, f\lceil\delta)=\rho_{X}(\delta)=1-\eta_{j}^{*}(\delta) .\right.\right.\right.
$$

But this contradicts the choice of $\eta_{j}^{*}$.

2) This corresponds to Case I, where we do not need the weak diamond, but just $2^{<\lambda}<2^{\lambda}$.

3),4),5). Check, similarly.

\subsection{Conclusion. 1) Assume}

$(*)_{1}$ for $\eta \in{ }^{\lambda>} 2, M_{\eta} \in K_{<\lambda}$ and $\left\langle M_{\eta\lceil\alpha}: \alpha \leq \ell g(\eta)\right\rangle$ is $\leq_{\mathfrak{K}^{-}}$-increasing continuous

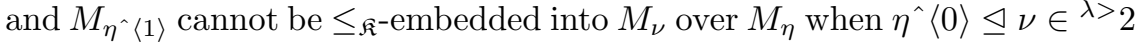
and $\left\langle M_{\eta}: \eta \in{ }^{\lambda>} 2\right\rangle$ is definable in $\mathfrak{B}$

$(*)_{2}^{d} \mathrm{WDmId}^{\operatorname{Def}}(\lambda)$ or $\operatorname{DfWD}^{+}(\lambda)$ is not $\lambda$-saturated (which holds if there is no normal $\lambda$-saturated ideals on $\lambda$ (which holds for non Mahlo $\lambda$ ) and holds if for some $\theta,\{\delta<\lambda: \operatorname{cf}(\delta)=\theta\}$ is not in the ideal).

Then the conclusion of 1.6 holds.

From the Definition below, we use here mainly "superlimit" 
1.8 Definition. 1) $M \in \mathfrak{K}_{\lambda}$ is a superlimit if

(a) for every $N \in \mathfrak{K}_{\lambda}$ such that $M \leq_{\mathfrak{K}} N$ there is $M^{\prime} \in K_{\lambda}$ such that $N \leq_{\mathfrak{K}}$ $M^{\prime}, N \neq M^{\prime}$, and $M \cong M^{\prime}$

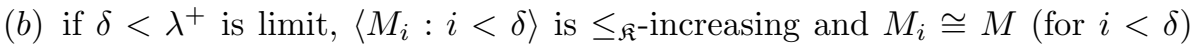
then $\bigcup_{i<\delta} M_{i} \cong M$.

2) For $\Theta \subseteq\left\{\mu: \aleph_{0} \leq \mu \leq \lambda, \mu\right.$ regular $\}$ we say $M \in \mathfrak{K}_{\lambda}$ is a $(\lambda, \Theta)$-superlimit if:

(a) clause $(\alpha)$ from part (1) holds and

(b) if $M_{i} \cong M$ is $\left(\leq_{\mathfrak{K}}\right)$-increasing for $i<\mu \in \Theta$ then $\bigcup_{i<\mu} M_{i} \cong M$.

3) For $S \subset \lambda^{+}$we say $M \in \mathfrak{K}_{\lambda}$ is a $(\lambda, S)$-strong limit if:

(a) clause $(\alpha)$ from part (1) holds

(b) there is a function $F$ from $\bigcup_{\alpha<\kappa}{ }^{\alpha}\left(K_{\lambda}\right)$ to $K_{\lambda}$ such that:

( $\alpha$ ) for any sequence $\left\langle M_{i}: i<\alpha\right\rangle$ if $\alpha<\kappa, M_{0}=M, M_{i}$ is $\leq_{\mathfrak{K}^{-}}$-increasing, and $M_{i} \in \mathfrak{K}_{\lambda}$, then $j<\alpha \Rightarrow M_{j} \leq_{\mathfrak{K}} F\left(\left\langle M_{i}: i<\alpha\right\rangle\right)$

( $\beta$ ) if $\left\langle M_{i}: i<\lambda^{+}\right\rangle$is $\leq_{\mathfrak{K}^{-}}$-increasing, $M_{0}=M, M_{i} \in \mathfrak{K}_{\lambda}$, and for $i<\kappa, M_{i+1} \leq_{\mathfrak{K}} F\left(\left\langle M_{j}: j \leq i+1\right\rangle\right) \leq_{\mathfrak{K}} M_{i+2}$ then $\left\{\delta \in S \mid \bigcup_{i<\delta} M_{i} \supsetneqq M\right\}$ is not stationary.

4) $M$ is a $(\lambda, \kappa)$-limit if there is a function $F$ as in $3 \mathrm{~b}(\alpha)$ such that:

(a) if $\left\langle M_{i}: i<\kappa\right\rangle$ is a $<_{\mathfrak{K}}$-increasing continuous sequence in $K_{\lambda}$, $F(\bar{M} \uparrow(i+1)) \leq_{\mathfrak{K}} M_{i+1}$ then $\bigcup_{i<\kappa} M_{i} \cong M$

(b) there is at least one such sequence.

5) $M$ is a $(\lambda, \kappa)$-superlimit is defined similarly, but with $F$ omitted and $M_{i+1} \cong M$.

1.9 Claim. 1) In 1.4(1) we can replace the categoricity of $\mathfrak{K}$ in $\chi$ by " $\mathfrak{K}$ has a super limit model in $\chi "$ which is not an amalgamation base (see Definition 1.8). In this case the assumption of 1.4(2), and of 1.6(1) holds.

2) We can weaken (in 1.6(5) the existence of superlimit to) "for some $\kappa=c f(\kappa) \leq$ $\chi$ there is a $(\chi,\{\kappa\})$-super limit model which is not an amalgamation base"; provided that we add $\{\delta<\lambda: c f(\delta)=\kappa\} \notin W D m I d_{<\mu}(\lambda)$ (but for 2.9(1) we need now "WDmId $<\mu(\lambda)+\{\delta<\lambda: c f(\delta)=\kappa\}$ is not $\lambda$-saturated". If there is $S \subseteq\{\delta<\lambda: c f(\delta)=\kappa\}$, which belongs to $I[\lambda]$ but not to $W D m I d_{<\mu}(\lambda)$ we can weaken the model theoretic requirement to: there is a $(\chi,\{\kappa\})$-medium limit (see [Sh 88, Definition §3]) but not used here. 
1.10 Claim. Assume $2^{\lambda}<2^{\lambda^{+}}$.

0) If $\mathfrak{K}$ (an abstract elementary class) is categorical in $\lambda$, $L S(\mathfrak{K}) \leq \lambda, I\left(\lambda^{+}, \mathfrak{K}\right)<$ $2^{\lambda^{+}}$, then $\mathfrak{K}_{\lambda}$ has amalgamation.

1) If $\mathfrak{K}$ (an abstract elementary class) is categorical in $\lambda$ and $1 \leq \operatorname{IE}\left(\lambda^{+}, K\right)<2^{\lambda^{+}}$ but $K_{\lambda^{++}}=\emptyset$ then $\mathfrak{K}$ has amalgamation in $\lambda$.

2) Assume $\mathfrak{K}$ has amalgamation in $\lambda, L S(\mathfrak{K}) \leq \lambda, K_{\lambda^{+}} \neq \emptyset$ and $K_{\lambda^{++}}=\emptyset$. Then there is $M \in K_{\lambda^{+}}$saturated above $\lambda$.

3) If $M$ is $\mu$-saturated above $\lambda, L S(\mathfrak{K}) \leq \lambda_{0}<\lambda$ and $\mathfrak{K}$ has amalgamation in every $\lambda_{0}^{\prime} \in\left[\lambda_{0}, \lambda\right)$ then $M$ is $\mu$-saturated above $\lambda_{0}$.

1.11 Remark. If $I\left(\lambda^{+}, \mathfrak{K}\right)<2^{\lambda^{+}}$, then the assumption $\mathfrak{K}_{\lambda^{++}}=\emptyset$ is not used in part (1) of 1.10; this is 1.6(1) $+(2)$. Also if $\left(2^{\lambda}\right)^{+}<2^{\lambda^{+}}$then the assumption $K_{\lambda^{++}}=\emptyset$ is not needed in part (1) of 1.10; by 1.6(1) + (2) (note (b) of 1.6(1)).

Proof. 0) By 1.6(1) applied to $\lambda^{+}$.

1) If not, we can choose for $\eta \in{ }^{\lambda^{+}}>2$ a model $M_{\eta} \in \mathfrak{K}_{\lambda}$ such that $\left[\nu \triangleleft \eta \Rightarrow M_{\nu} \leq_{\mathfrak{K}} M_{\eta}\right]$, and $M_{\eta^{\wedge}\langle 0\rangle}, M_{\eta^{\wedge}\langle 1\rangle}$ cannot be amalgamated over $M_{\eta}$. If $\left(2^{\lambda}\right)^{+}<2^{\lambda^{+}}$we are done by 1.6(1), so assume $\left(2^{\lambda}\right)^{+}=2^{\lambda^{+}}$. For each $\eta \in{ }^{\lambda^{+}} 2$ let $M_{\eta}=: \bigcup_{\alpha<\lambda^{+}} M_{\eta\lceil\alpha}$, and let $N_{\eta} \in \mathfrak{K}_{\lambda^{+}}$be such that

$M_{\eta} \leq_{\mathfrak{K}} N_{\eta}, N_{\eta}$ is $\leq_{\mathfrak{K}^{-}}$maximal (exists as $\mathfrak{K}_{\lambda^{++}}=\emptyset$ ). Now we choose by induction $\zeta<\left(2^{\lambda}\right)^{+}, \eta_{\zeta} \in{ }^{\lambda^{+}} 2$ such that $M_{\eta_{\zeta}}$ is not $\leq_{\mathfrak{K}^{-}}$-embeddable into $N_{\eta_{\xi}}$ for $\xi<\zeta$ (exists by 1.4(2)). So necessarily for $\xi<\zeta, N_{\eta_{\zeta}}$ is not $\leq_{\mathfrak{K}^{-}}$-embeddable into $N_{\eta_{\xi}}$ (as $M_{\eta_{\zeta}} \leq N_{\eta_{\zeta}}$ ). Also, for $\xi<\zeta, N_{\eta_{\xi}}$ is not $\leq_{\mathfrak{K}^{-}}$-embeddable into $N_{\eta_{\zeta}}$ as otherwise by the maximality of $N_{\xi}$ this implies $N_{\xi} \cong N_{\zeta}$. So $\left\{N_{\zeta}: \zeta<2^{\lambda^{+}}\right\}$exemplifies $\operatorname{IE}\left(\lambda^{+}, \mathfrak{K}\right)=2^{\lambda^{+}}$, contradicting an assumption.

2) A maximal model in $K_{\lambda^{+}}$will do.

3) Easy.

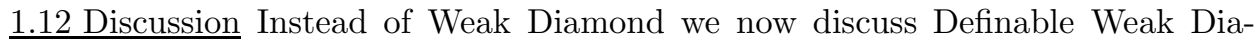
mond, which is weaker but suffices.

Compare [MkSh 313], where many Cohen subsets are added to $\lambda$ and a combinatorial principle about amalgamation of configurations $\left\langle M_{s}: s \subseteq n, s \neq n\right\rangle$ is gotten.

We are interested here in the case $n=1$ (ordinary amalgamation); in $\S 3$, also $n=2$. Even more definability can be required.

This is particularly interesting when we look at results under some other set theory, when combining $2^{\lambda}=2^{\lambda^{+}}$with definable weak diamond on $\lambda^{+}$is helpful. This played a major role in the preliminary form of this work.

1.13 Definition. 1) In Definition 1.1 we add the superscript $\mathscr{F}$ if we restrict ourselves to functions $F \in \mathscr{F}$.

2) Fix a model $\mathfrak{B}$ whose universe includes $\lambda$ and has a definable pairing function on $\lambda$, and a logic $\mathscr{L}$ closed under first order operations and substitution; also allow " $M \in \mathfrak{K}$ " and " $M \leq_{\mathfrak{K}} N$ " in the formulas, if it is not said otherwise. Let 


$$
\begin{aligned}
\mathscr{F}_{\mathfrak{B}, \mathscr{L}}^{\text {Def }}=\{F & \text { for some } g \in{ }^{\lambda} \lambda \text { and } \bar{h}=\left\langle h_{\eta}: \eta \in{ }^{\lambda>} 2\right\rangle \text { where } \\
& h_{\eta}: \ell g(\eta) \rightarrow \lambda \text { and } h_{\eta \uparrow \beta} \subseteq h_{\eta} \text { for } \beta<\ell g(\eta) \\
& \text { and for some sequence } \bar{\psi}=\left\langle\psi_{\alpha}: \alpha<\lambda\right\rangle \text {, with } \\
& \psi_{\alpha} \in \mathscr{L} \text { for } \alpha<\lambda \text { the following holds for every } \alpha<\lambda \text { and } \\
& \left.f \in{ }^{\alpha}\left(2^{<\lambda}\right): F(f)=1 \text { iff }\left(\mathfrak{B}, \alpha, g, h_{f}\right) \models \psi_{\alpha} \text { iff } F(f) \neq 0\right\} .
\end{aligned}
$$

3) The version of weak diamond from 1.1, restricted to the class $\mathscr{F}$ of 1.13(2) is called the $(\mathfrak{B}, \mathscr{L})$-definitional version. If $\mathscr{L}$ is $\mathscr{L}_{\lambda, \lambda}$ we may omit it. If $\mathfrak{B}$ has the form $\left(\mathscr{H}(\chi), \in,<_{\chi}^{*}, \lambda\right)$ we write $\mathscr{F}_{\mathscr{L}}^{\mathrm{Def}[(\chi)]}$ or $\mathscr{F}^{\operatorname{Def}(\chi)}$ instead of $\mathscr{F}_{\mathfrak{B}, \mathscr{L}}^{\mathrm{Def}}$ or $\mathscr{F}_{\mathfrak{B}}^{\mathrm{Def}}$ respectively. If we omit $\chi$, we mean $\chi=\left(2^{\lambda}\right)^{+}$and we may put $\operatorname{Def}(\chi)$ or $\operatorname{Def}$ instead of $\mathscr{F}^{\operatorname{Def}(\chi)}$ or $\mathscr{F}^{\text {Def }}$ in the superscript. Having the definitional version or the definable weak diamond for $\lambda$ means $\lambda \notin \operatorname{WDmId}^{\mathrm{Def}}(\lambda)$.

4) Let $\operatorname{DfWD}_{<\mu}(\lambda)$ mean that with $\mathfrak{B}=\left(\mathscr{H}(\chi), \in,<_{\chi}^{*}, \lambda, \mu\right)$ we have $\lambda \notin \operatorname{WDmId}_{<\lambda}^{\text {Def }}(\lambda)$. Instead of " $<\mu^{+}$" we write " $\mu$ " and instead of " $<2^{<\lambda^{+}}$" we may write nothing. 5) Let $\operatorname{DfWD}_{<\mu}^{+}(\lambda)$ mean $\operatorname{DfWD}_{<\mu}(\lambda)$ together with the principle $\otimes_{\lambda}$ below; we adopt the same conventions as in (4) concerning $\mu$ :

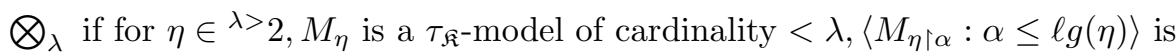
s-increasing continuous, for $\eta \in{ }^{\lambda} 2$ we let $M_{\eta}=\bigcup_{\alpha<\lambda} M_{\eta\lceil\alpha}$ and for $\eta \neq \nu \in{ }^{\lambda} 2, M_{\eta}$ and $M_{\nu}$ are not isomorphic over $M_{\langle\rangle}$, then $\left\{M_{\eta} \cong: \eta \in{ }^{\lambda} 2\right\}$ has cardinality $2^{\lambda}$ (note that $2^{<\lambda}<2^{\lambda}$ implies that).

\subsection{Claim.}

1) Assume $\mathscr{L}$ first order or at least definable enriching first order.

In the definition of $\mathscr{F}_{\mathfrak{B}, \mathscr{L}}^{\mathrm{Def}}$, we can replace "for every $\alpha<\lambda$ " by "for a club of $\alpha<\lambda^{\prime}$. In the definition of $\mathscr{F}$ Def we can let $g \in{ }^{\lambda}(\lambda>2)$ and $h_{\eta}: \ell g(\eta) \rightarrow^{\lambda>} 2$. In any case $W D m I d_{<\mu}^{\mathscr{F}}(\lambda)$ increases with $\mathscr{F}$ and is $\subseteq W D m I d_{<\mu}(\lambda)$, similarly for $W D m T I d_{<\mu}^{\mathscr{F}}(\lambda)$.

2) If $\mathscr{F}=\mathscr{F}_{\mathfrak{B}}^{\mathrm{Def}}$ and $c f(\mu)>\lambda \underline{\text { then }} \operatorname{WDmId}_{<\mu}(\lambda)$ is a normal ideal (but possibly is equal to $\mathscr{P}(\lambda)$ ).

3) Assume $V \models " \lambda=\chi^{+}, \chi^{<\chi}=\chi, \mu>\lambda$ " and $P$ is the forcing notion of adding $\mu$ Cohen subsets to $\chi$ (i.e.

$\{g: g$ a partial function from $\mu$ to $\{0\}$ with domain of cardinality $<\chi\})$.

$\underline{\text { Then in }} V^{P}$ we have $W D m I d_{<\mu}^{\mathrm{Def}}(\lambda)$ is the ideal of non-stationary subsets of $\lambda$; i.e. with

$\mathfrak{B}=\left(\mathscr{H}(\chi), \in,<_{\mu}^{*}\right)^{V^{P}}$ for any $\chi$. Also $\otimes_{\lambda}$ of Definition 1.13(5) holds.

Remark. In 1.14(1) we use the assumption on $\mathscr{L}$; anyhow not serious: reread the definition 1.1(1). 
Proof. 1); 2) By manipulating the $h$ 's (using the pairing function on $\lambda$ ).

3) See [MkSh 313] or think (the point being that we can break the forcing, first adding $\bar{\psi}$ and $g$ (or the $<\mu$ ones) and then (read 1.1(1)) choose $\eta \in{ }^{\lambda} 2$ as

$g \uparrow[\gamma, \gamma+\lambda)$ not "used before". Now for any candidate $f \in{ }^{\lambda}\left({ }^{\lambda>} 2\right)$ for a club of $\widetilde{\delta}<\lambda, \eta(\delta)=g(\gamma+\delta)$ is not used in the definition of $f \uparrow \delta, h_{f} \uparrow \delta$ so stationarily often $\eta(\delta)$ "guesses" rightly.)

1.15 Claim. 1) If $2^{\theta}=2^{<\lambda}<2^{\lambda}$ then $D f W D^{+}(\lambda)$.

2) $D f W D_{<\mu}(\lambda)$ holds when $\lambda \notin W D m I d_{<\mu}(\lambda)$ (see 1.2 for sufficient conditions).

1.16 Discussion: We hope to get successful "guessing" not just on a stationary set, but on a positive set for the same ideal for which have guessed; i.e. there is $I$ a normal ideal on $\lambda$ such that for $A \in I^{+}$there is $\eta \in{ }^{\zeta} 2$ guessing $I$-positively; this is connected to questions on $\lambda^{+}$-saturation. For more see [Sh 638].

We phrased the following notion originally in the hope of later eliminating $\mu_{\mathrm{wd}}(\lambda)$ (i.e. using $2^{\lambda}$ instead of $\mu_{\mathrm{wd}}(\lambda)$ ).

1.17 Definition. 1)

$$
\begin{aligned}
\operatorname{UDmId}_{<\mu}^{\mathscr{F}}(\lambda)=\{S \subseteq \lambda: & \text { for some } i^{*}<\mu \text { and } F_{i} \in \mathscr{F}\left(\text { for } i<i^{*}\right) \\
& \text { for every } \eta \in{ }^{S} 2 \text { there is } f \in{ }^{\lambda}\left(2^{<\lambda}\right) \text { and } i<i^{*} \\
& \text { and club } E \text { of } \lambda \text { such that: } \\
& \text { for every } \delta \in E \text { we have: } \\
& \delta \in S \Rightarrow \eta(\delta)=F_{i}(f\lceil\delta) \\
& \delta \in \lambda \backslash S \Rightarrow 0=F_{i}(f\lceil\delta)\} .
\end{aligned}
$$

2) We omit $\mu$ if $\mu=1$.

3) $B A^{\mathscr{F}}(\lambda)$ is defined as the family of $S \subseteq \lambda$ such that for some $F \in \mathscr{F}$ and $\eta=O_{S}$ the condition above holds.

1.18 Claim. Assume $\mathscr{F}=\mathscr{F}_{\mathfrak{B}}^{\text {Def }}$.

1) In the definition 1.17(1) we can replace $f \in{ }^{\lambda}\left(2^{<\lambda}\right)$ by $f \in{ }^{\lambda} 2$ or $f \in{ }^{\lambda}\left({ }^{\lambda>} 2\right)$.

2) $\operatorname{UDmId} d^{\mathscr{F}}(\lambda)$ is a normal ideal on $\lambda$ (but possibly is $\mathscr{P}(\lambda)$ ).

3) $B A^{\mathscr{F}}(\lambda)$ is a Boolean algebra of subsets of $\lambda$ including all non-stationary subsets of $\lambda$ and even $U D m I d^{\mathscr{F}}(\lambda)$, and is closed under unions of $<\lambda$ sets and even under diagonal union.

4) If $S \in B A^{\mathscr{F}}(\lambda)$ and $F \in \mathscr{F}$ then for some $\eta \in S_{2}$ we have:

(*) for every $f \in^{\lambda}\left({ }^{\lambda}>2\right)$ we have

$$
\left\{\delta \in S: \eta(\delta)=F(f\lceil\delta)\} \neq \emptyset \bmod \operatorname{UDmId}^{\mathscr{F}}(\lambda) .\right.
$$


5) $U D m I d_{<\mu}^{\mathscr{F}}(\lambda) \subseteq W D m I d_{<\mu}^{\mathscr{F}}(\lambda) \subseteq W D m I d_{<\mu}(\lambda)$ and they increase with $\mathscr{F}$ and $\lambda \in U D m I d_{<\mu}^{\mathscr{F}}(\lambda) \Leftrightarrow \lambda \in W D m I d_{<\mu}^{\mathscr{F}}(\lambda)$.

Proof. Straightforward.

1.19 Discussion Remember

$(*)_{1}$ If $V=" \chi=\chi^{<\chi} \quad \& \quad 2^{\chi}=\chi^{+} ", P$ is the forcing notion of adding $\mu>\chi^{+}$ Cohen subsets to $\chi \underline{\text { then }}$ in $V^{\chi}$, any equivalence relation on $\mathscr{P}(\lambda)$ definable with parameters $X \subseteq \chi$ and ordinals which has at least $\chi^{++}$equivalence classes has at least $\mu$ equivalence classes

and (see [Sh:f, XVI,§2])

$(*)_{2}$ ZFC is consistent with $C H+$ for some stationary, costationary $S \subseteq \omega_{1}$ we have

(a) $\operatorname{WDmId}\left(\aleph_{1}\right)=\left\{A \subseteq \omega_{1}: A \backslash S\right.$ is not stationary $\}$

(b) $\mathscr{D}_{\omega_{1}}+S$ is $\aleph_{2}$-saturated.

$(*)_{3} Z F C+G C H$ is consistent with $\left\{\delta<\aleph_{2}: \operatorname{cf}(\delta)=\aleph_{1}\right\} \in \operatorname{WDmId}\left(\aleph_{2}\right)$

$(*)_{4} Z F C+2^{\aleph_{1}}<2^{\aleph_{2}}$ is consistent with $\left\{\delta<\aleph_{2}: \operatorname{cf}(\delta)=\aleph_{0}\right\} \in \operatorname{WDmId}\left(\aleph_{2}\right)$. 


\section{$\S 2$ First ATtempts}

Given amalgamation in $K_{\lambda}$ (cf. 2.2) we try to define and analyze types $p \in \mathscr{S}(M)$ for $M \in K_{\lambda}$. But types here (as in [Sh 300]) are not sets of formulas. They may instead be represented by triples $(M, N, a)$ with $M \leq_{\mathfrak{K}} N$ and $a \in N \backslash M$. We look for nice types (i.e. triples) and try to prove mainly the density of the set of minimal types).

To simplify matters we allow uses of stronger assumptions than are ultimately desired (e.g. $2^{\lambda^{+}}>\lambda^{++}$and/or $K_{\lambda^{+3}}=\emptyset$ ). These will later be eliminated. However the first extra assumption is still a "mild set theoretic assumption", and the second is harmless if we think only of proving our main theorem 0.2 and not on subsequent continuations.

So the aim of this section is to show that we can start to analyze such classes.

2.1 Hypothesis. $\mathfrak{K}$ is an abstract elementary class.

2.2 Claim. Assume

$(*)_{\lambda}^{2} K$ is categorical in $\lambda ; 1 \leq I\left(\lambda^{+}, K\right)<2^{\lambda^{+}} ; L S(\mathfrak{K}) \leq \lambda$ and: $2^{\lambda}<2^{\lambda^{+}}$, or at least the definable weak diamond holds for $\lambda^{+}$holds.

$\underline{\text { Then }}$

1) $\mathfrak{K}_{\lambda}$ has amalgamation.

2) If $I\left(\lambda^{++}, K\right)=0$ then $\mathfrak{K}$ has a model in $\lambda^{+}$which is universal homogeneous above $\lambda$, hence saturated above $\lambda$ (see 0.22(2)).

3) If $I\left(\lambda^{++}, K\right)=0$ then $M \in K_{\lambda} \Rightarrow|\mathscr{S}(M)| \leq \lambda^{+}$.

Proof. 1) If amalgamation fails in $K_{\lambda}$ and $2^{\lambda}<2^{\lambda^{+}}$, then the assumptions of 1.4(1) hold with $\lambda^{+}$in place of $\lambda$. Hence by 1.6(2) the statement $(*)_{1}$ of 1.6(1) (see there) holds and easily also $(*)_{2}$ of $1.6(1)$, hence by $1.6(4)$ we have $I\left(\lambda^{+}, K\right)=2^{\lambda^{+}}$, a contradiction. If $2^{\lambda}=2^{\lambda^{+}}$, we are using the variants from 1.13 .

2) As $I\left(\lambda^{++}, K\right)=0<I\left(\lambda^{+}, K\right)$, there is $M \in K_{\lambda^{+}}$which is maximal. If $M$ is not universal homogeneous above $\lambda$ then there are $N_{0}, N_{1} \in K_{\lambda}$ with $N_{0} \leq_{\mathfrak{K}} M$ and $N_{0} \leq_{\mathfrak{K}} N_{1}$ such that $N_{1}$ cannot be $\leq_{\mathfrak{K}}$-embedded into $M$ over $N_{0}$. Use $0.20(2)$ to get a contradiction.

3) Follows from (2).

\subsection{Definition.}

1)(a) $K_{\lambda}^{3}=\left\{\left(M_{0}, M_{1}, a\right): M_{0} \leq_{\mathfrak{K}} M_{1}\right.$ are both in $K_{\lambda}$ and $\left.a \in M_{1} \backslash M_{0}\right\}$.

$$
\left(M_{0}, M_{1}, a\right) \leq\left(M_{0}^{\prime}, M_{1}^{\prime}, a^{\prime}\right) \text { if } a=a^{\prime}, M_{0} \leq_{\mathfrak{K}} M_{0}^{\prime}, M_{1} \leq_{\mathfrak{K}} M_{1}^{\prime}
$$

(c) $\left(M_{0}, M_{1}, a\right) \leq_{h}\left(M_{0}^{\prime}, M_{1}^{\prime}, a^{\prime}\right)$ if $h(a)=a^{\prime}$, and for $\ell=0,1$ we have: $h \uparrow M_{\ell}$ is a $\leq_{\mathfrak{K}}$-embedding of $M_{\ell}$ into $M_{\ell}^{\prime}$. 


$$
\begin{aligned}
\left(M_{0}, M_{1}, a\right)< & \left(M_{0}^{\prime}, M_{1}^{\prime}, a^{\prime}\right) \text { if }\left(M_{0}, M_{1}, a\right) \\
& \leq\left(M_{0}^{\prime}, M_{1}^{\prime}, a\right) \text { and } M_{0} \neq M_{0}^{\prime} .
\end{aligned}
$$

$$
\text { similarly }<_{h}
$$

2) $\left(M_{0}, M_{1}, a\right) \in K_{\lambda}^{3}$ has the weak extension property if there is $\left(M_{0}^{\prime}, M_{1}^{\prime}, a\right) \in K_{\lambda}^{3}$ such that $\left(M_{0}, M_{1}, a\right) \leq\left(M_{0}^{\prime}, M_{1}^{\prime}, a\right)$ and $M_{0} \neq M_{1}$.

3) $\left(M_{0}, M_{1}, a\right) \in K_{\lambda}^{3}$ has the extension property if: for every $N_{0} \in \mathfrak{K}_{\lambda}$ and $\leq_{\mathfrak{K}}$-embedding $f$ of $M_{0}$ into $N_{0}$ there are $N_{1}, b$ and $g$ such that: $\left(M_{0}, M_{1}, a\right) \leq_{g}$ $\left(N_{0}, N_{1}, b\right) \in K_{\lambda}^{3}$ and $g \supseteq f$ (so $g(a)=b$ and $g$ is a $\leq_{\mathfrak{K}}$-embedding of $M_{1}$ into $N_{1}$ ).

\subsection{Claim. Assume}

$(*)_{\lambda}^{3} L S(\mathfrak{K}) \leq \lambda, K$ is categorical in $\lambda$ and in $\lambda^{+}$, and $1 \leq I\left(\lambda^{++}, K\right)$.

$\underline{\text { Then every }}\left(M_{0}, M_{1}, a\right) \in K_{\lambda}^{3}$ has the weak extension property that is:

If $M_{0} \leq_{\mathfrak{K}} M_{1}$ are in $K_{\lambda}$ and $a \in M_{1} \backslash M_{0}$, then we can find $M_{1}^{\prime}$ in $\mathfrak{K}_{\lambda}$ such that: $M_{0}<_{\mathfrak{K}} M_{1}$ hence $M_{0} \neq M_{0}^{\prime}$ and $\left(M_{0}, M_{1}, a\right) \leq\left(M_{0}^{\prime}, M_{1}^{\prime}, a\right)$.

Proof. We can choose $\left\langle N_{i}, a_{i}: i<\lambda^{+}\right\rangle$such that:

(a) $N_{i} \in K_{\lambda}$ is $\leq_{\mathfrak{K}}$-increasing continuous in $i$;

(b) $h_{i}$ is an isomorphism from $M_{1}$ onto $N_{i+1}$ such that

$$
h_{i}\left(M_{0}\right)=N_{i}, h_{i}(a)=a_{i} \text {. }
$$

Now as $a \in M_{1} \backslash M_{0}$ clearly $i<j<\lambda^{+} \Rightarrow a_{i} \in N_{i+1} \leq_{\mathfrak{K}} N_{j} \& a_{j} \notin N_{i+1}$ hence $\bigcup_{i<\lambda^{+}} N_{i} \in K_{\lambda^{+}}$.

By $0.20(1)$ applied to $\lambda^{+}$there are $M_{0}^{\prime} \leq_{\mathfrak{K}} M_{1}^{\prime}$ in $K_{\lambda^{+}}, M_{0}^{\prime} \neq M_{1}^{\prime}$, and there is $b \in M_{1}^{\prime} \backslash M_{0}^{\prime}$. As $K$ is categorical in $\lambda^{+}$, without loss of generality $M_{0}^{\prime}=\bigcup_{i<\lambda^{+}} N_{i}^{\prime}$.

Let $\chi$ be large enough and $\mathfrak{B} \prec\left(\mathscr{H}(\chi) \in,<_{\chi}^{*}\right)$ be such that $\lambda \subseteq \mathfrak{B},\|\mathfrak{B}\|=\lambda$ and $\left\langle N_{i}, a_{i}: i<\lambda^{+}\right\rangle, M_{0}^{\prime}, M_{1}^{\prime}, b$ and the definition of $\mathfrak{K}$ belong to $\mathfrak{B}$.

Let $\delta=\mathfrak{B} \cap \lambda^{+}$, so $\delta \in\left(\lambda, \lambda^{+}\right)$is a limit ordinal and

$$
\begin{gathered}
N_{\delta} \leq_{\mathfrak{K}} N_{\delta+1} \leq_{\mathfrak{K}} M_{1}^{\prime}, \\
N_{\delta} \leq_{\mathfrak{K}}\left(M_{1}^{\prime} \cap \mathfrak{B}\right) \leq_{\mathfrak{K}} M_{1}^{\prime} \\
\mathfrak{B} \cap M_{0}^{\prime}=N_{\delta} \\
N_{\delta+1} \cap\left(M_{1}^{\prime} \cap \mathfrak{B}\right)=N_{\delta},
\end{gathered}
$$


so for some $N$ we have:

$$
N \in \mathfrak{K}_{\lambda}, N \leq_{\mathfrak{K}} M_{1}^{\prime} \text {, and }\left(N_{\delta+1} \cup\left(M_{1}^{\prime} \cap \mathfrak{B}\right)\right) \subseteq N
$$

so (see Definition 2.3(1) above)

$$
\left(N_{\delta}, N_{\delta+1}, a_{\delta}\right) \leq_{\mathfrak{K}}\left(M_{1}^{\prime} \cap \mathfrak{B}, N, a_{\delta}\right)
$$

and $b$ witnesses $N_{\delta} \neq M_{1}^{\prime} \cap \mathfrak{B}$.

As $\left(M_{0}, M_{1}, a\right) \cong\left(N_{\delta}, N_{\delta+1}, a_{\delta}\right)$, the result follows $)$.

2.5 Definition. 1) $\left(M_{0}, M_{1}, a\right) \in K_{\lambda}^{3}$ is $\underline{\text { minimal when: }}$

$$
\begin{gathered}
\text { if }\left(M_{0}, M_{1}, a\right) \leq_{h_{\ell}}\left(M_{0}^{\prime}, M_{1}^{\ell}, a_{\ell}\right) \in K_{\lambda}^{3} \text { for } \ell=1,2, \\
\text { then } \operatorname{tp}\left(a_{1}, M_{0}^{\prime}, M_{1}^{1}\right)=\operatorname{tp}\left(a_{2}, M_{0}^{\prime}, M_{1}^{2}\right) .
\end{gathered}
$$

2) $\left(M_{0}, M_{1}, a\right) \in K_{\lambda}^{3}$ is reduced when:

$$
\text { if }\left(M_{0}, M_{1}, a\right) \leq\left(M_{0}^{\prime}, M_{1}^{\prime}, a\right) \in \mathfrak{K}_{\lambda}^{3} \text { then } M_{0}^{\prime} \cap M_{1}=M_{0} \text {. }
$$

3) We say $p \in \mathscr{S}\left(M_{0}\right)$ is $\underline{\text { minimal }}$, where $M_{0} \in K_{\lambda}$, if for some $a, M_{1}$ we have: $p=\operatorname{tp}\left(a, M_{0}, M_{1}\right)$ and $\left(M_{0}, M_{1}, a\right) \in K_{\lambda}^{3}$ is minimal.

4) We say $p \in \mathscr{S}\left(M_{0}\right)$ is reduced where $M_{0} \in K_{\lambda}$, if for some $a, M_{1}$ we have $p=\operatorname{tp}\left(a, M_{0}, M_{1}\right)$ and $\left(\overline{M_{0}, M_{1}}, a\right) \in K_{\lambda}^{3}$ is reduced.

2.6 Fact. 1) For every $\left(M_{0}, M_{1}, a\right) \in K_{\lambda}^{3}$ there is a reduced $\left(M_{0}^{\prime}, M_{1}^{\prime}, a\right)$ such that: $\left(M_{0}, M_{1}, a\right) \leq\left(M_{0}^{\prime}, M_{1}^{\prime}, a\right) \in K_{\lambda}^{3}$.

2) Assume $\left\langle\left(M_{0, \alpha}, M_{1, \alpha}, a\right): \alpha<\delta\right\rangle$ is an increasing sequence of members of $K_{\lambda}^{3}$

$(a)$ if $\delta<\lambda^{+}$then $\left(M_{0, \alpha}, M_{1, \alpha}, a\right) \leq\left(\bigcup_{\beta<\delta} M_{0, \beta}, \bigcup_{\beta<\delta} M_{1, \beta}, a\right) \in K_{\lambda}^{3}$.

(b) If $\delta=\lambda^{+}$the result may be in $K_{\lambda^{+}}^{3}$ : if $\left\{\alpha<\delta: M_{0, \alpha} \neq M_{0, \alpha+1}\right\}$ is cofinal, this holds.

(c) If $\delta<\lambda^{+}$and each $\left(M_{0, \alpha}, M_{1, \alpha}, a\right)$ is reduced then so is $\left(\bigcup_{\beta<\delta} M_{0, \beta}, \bigcup_{\beta<\delta} M_{1, \beta}, a\right)$.

3) If $\left(M_{0}, M_{1}, a\right) \leq\left(M_{0}^{\prime}, M_{1}^{\prime}, a\right)$ are in $K_{\lambda}^{3}$ and the first triple is minimal then so is the second.

4) If $\left(M_{0}, M_{1}, a\right) \leq\left(M_{0}^{\prime}, M_{1}^{\prime}, a\right)$ are in $K_{\lambda}^{3} \underline{\text { then }} \operatorname{tp}\left(a, M_{0}, M_{1}\right) \leq \operatorname{tp}\left(a, M_{0}^{\prime}, M_{1}^{\prime}\right)$; (Definition 0.24(1)).

5) If $K_{\lambda}$ has amalgamation, then: $\left(M_{0}, M_{1}, a\right) \in K_{\lambda}^{3}$ is minimal if and only if:

(*) If $\left(M_{0}, M_{1}, a\right) \leq_{h_{\ell}}\left(M_{0}^{\prime}, M_{1}^{\prime}, a_{\ell}\right) \in K_{\lambda}^{3}$ for $\ell=1,2$ and $h_{1} \uparrow M_{0}=h_{2} \uparrow M_{0}$ then $\operatorname{tp}\left(a_{1}, M_{0}^{\prime}, M_{1}^{\prime}\right)=\operatorname{tp}\left(a_{2}, M_{0}^{\prime}, M_{1}^{\prime}\right)$. 
6) If there is no maximal member ${ }^{2}$ of $K_{\lambda}^{3}$ and there are $N_{0}<_{\mathfrak{K}} N_{1}$ in $K_{\lambda}$, then there are $N^{0}<_{\mathfrak{K}} N^{1}$ in $\mathfrak{K}_{\lambda^{+}}$.

7) If every triple in $K_{\lambda}^{3}$ has the weak extension property, and there are $N_{0}<_{\mathfrak{K}} N_{1}$ in $\mathfrak{K}_{\lambda}$ then there are $N^{0}<_{\mathfrak{K}} N^{1}$ in $\mathfrak{K}_{\lambda^{+}}$.

8) If $\overline{\mathrm{LS}(\mathfrak{K})} \leq \lambda$ and every triple in $K_{\lambda}^{3}$ has the extension property and $K_{\lambda}^{3} \neq \emptyset \underline{\text { then }}$ no $M \in K_{\lambda^{+}}$is $<_{\mathfrak{K}^{-}}$-maximal hence $K_{\lambda^{++}} \neq \emptyset$.

9) If $L S(\mathfrak{K}) \leq \lambda$ and $K_{\lambda^{+}} \neq \emptyset$, then $K_{\lambda}^{3} \neq \emptyset$.

Proof. Easy. Note that part (7) is 2.4 .

2.7 Claim. Assume $(*)_{\lambda^{+}}^{2}+(*)_{\lambda}^{3}$ (i.e. the hypothesis of 2.2 and 2.4) and $2^{\lambda^{+}}>$ $\lambda^{++}$, and $K_{\lambda+3}=\emptyset$.

Then in $\mathfrak{K}_{\lambda}^{3}$ the minimal triples are dense (i.e. above every triple in $K_{\lambda}^{3}$ there is a minimal one).

Remark. We do not intend to adopt the hypotheses " $2^{\lambda^{+}}>\lambda^{++}$", $K_{\lambda^{+3}}=\emptyset$ indefinitely. They will be eliminated in $\S 3$.

Proof. If not, we can choose by induction on $\alpha<\lambda^{+}$, for $\eta \in{ }^{\alpha} 2$ a triple $\left(M_{\eta}^{0}, M_{\eta}^{1}, a_{\eta}\right)$ and $h_{\eta, \nu}$ for $\nu \unlhd \eta$ such that:

(i) $\left(M_{\eta}^{0}, M_{\eta}^{1}, a_{\eta}\right) \in K_{\lambda}^{3}$

(ii) $\nu \triangleleft \eta \Rightarrow\left(M_{\nu}^{0}, M_{\nu}^{1}, a_{\nu}\right) \leq_{h_{\eta, \nu}}\left(M_{\eta}^{0}, M_{\eta}^{1}, a_{\eta}\right)$

(iii) $\nu_{0} \triangleleft \nu_{1} \triangleleft \nu_{2} \Rightarrow h_{\nu_{2}, \nu_{0}}=h_{\nu_{2}, \nu_{1}} \circ h_{\nu_{1}, \nu_{0}}$

(iv) $\left(M_{\eta^{\wedge}\langle\ell\rangle}^{0}, M_{\eta^{\wedge}\langle\ell\rangle}^{1}, h_{\eta^{\wedge}\langle\ell\rangle, \eta}\left\lceil M_{\eta}^{0}\right)\right.$ for $\ell=0,1$ are equal

(v) $\operatorname{tp}\left(a_{\eta^{\wedge}\langle 0\rangle}, M_{\eta^{\wedge}\langle 0\rangle}^{0}, M_{\eta^{\wedge}\langle 0\rangle}^{1}\right) \neq \operatorname{tp}\left(a_{\eta^{\wedge}\langle 1\rangle}, M_{\eta^{\wedge}\langle 1\rangle}^{0}, M_{\eta^{\wedge}\langle 1\rangle}^{1}\right) ;$

this makes sense as $M_{\eta^{\wedge}\langle 0\rangle}^{0}=M_{\eta^{\wedge}\langle 1\rangle}^{0}$

(vi) if $\eta \in{ }^{\delta} 2$ and $\delta<\lambda^{+}$is a limit ordinal, then $M_{\eta}^{\ell}=\bigcup_{\alpha<\delta} h_{\eta, \eta\lceil\alpha}\left(M_{\eta\lceil\alpha}^{\ell}\right)$ for $\ell=0,1$,

(vii) $\left(M_{<>}^{0}, M_{<>}^{1}, a_{<>}\right) \in K_{\lambda}^{3}$ is a triple above which there is no minimal one.

This is straightforward: for $\alpha=0$ choose a triple in $K_{\lambda}^{3}$ above which supposedly there is no minimal triple; in limit $\alpha$ take limits of diagrams (chasing the $h$ 's); in successor $\alpha$, use non-minimality and 2.6(5).

Let $M^{*} \in \mathfrak{K}_{\lambda^{+}}$be saturated above $\lambda$ (exists by $2.2(2)$ so it is necessarily homogeneous universal above $\lambda^{+}$, hence above $\lambda$; note: $\lambda$ there stands for $\lambda^{+}$here).

We choose by induction on $\alpha<\lambda^{+}$for $\eta \in{ }^{\alpha} 2$, a $\leq_{\mathfrak{K}^{-}}$-embedding $g_{\eta}$ of $M_{\eta}^{0}$ into $M^{*}$ such that:

$$
\begin{gathered}
\nu \triangleleft \eta \Rightarrow g_{\nu}=g_{\eta} \circ h_{\eta, \nu} . \\
g_{\eta^{\wedge}\langle 0\rangle}=g_{\eta^{\wedge}\langle 1\rangle} .
\end{gathered}
$$

\footnotetext{
${ }^{2}$ will be applied for $\lambda^{+}$
} 
This is clearly possible. Let $N_{\eta}^{0}=M^{*} \uparrow \operatorname{Rang}\left(g_{\eta}\right)$. For $\eta \in{ }^{\lambda^{+}} 2$ let $N_{\eta}^{0}=M^{*} \uparrow$ $\bigcup_{\alpha<\lambda^{+}} \operatorname{Rang}\left(g_{\eta\lceil\alpha}\right)$ and let $g_{\eta}=\bigcup_{\alpha<\lambda^{+}} g_{\eta\lceil\alpha}$. Chasing arrows we can find for $\eta \in{ }^{\lambda^{+}} 2$ a limit to $\left(\left\langle M_{\eta\lceil\alpha}^{0}, M_{\eta\lceil\alpha}^{1}, a_{\eta\lceil\alpha}\right\rangle, h_{\eta\lceil\beta, \eta\lceil\alpha}: \alpha<\beta<\lambda^{+}\right)$say $\left(M_{\eta}^{0}, M_{\eta}^{1}, a_{\eta}\right) \in K_{\lambda^{+}}^{3}$ and $h_{\eta, \nu}$ for $\nu \triangleleft \eta$ as usual. Let $f_{\eta}$ be the function from $M_{\eta}^{0}$ into $M^{*}$ such that for $\alpha<\lambda^{+}$we have $f_{\eta} \circ h_{\eta, \eta\lceil\alpha}=g_{\eta}$. So $f_{\eta}$ is a $\leq_{\mathfrak{K}^{-}}$embedding of $M_{\eta}^{0}$ into $M^{*}$. So we can extend $f_{\eta}$ to $f_{\eta}^{+}$, a $\leq_{\mathfrak{K}^{-}}$-embedding of $M_{\eta}^{1}$ into $M^{*}$.

Let $a_{\eta}^{*}=f_{\eta}^{+}\left(a_{\eta}\right)$ for $\eta \in \lambda^{\lambda^{+}} 2$.

As $2^{\lambda^{+}}>\lambda^{++}$for some $\eta_{0} \neq \eta_{1}$ we have $a_{\eta_{0}}^{*}=a_{\eta_{1}}^{*}$. So for some $\alpha<\lambda^{+}$, $\eta_{0}\left\lceil\alpha=\eta_{1}\left\lceil\alpha\right.\right.$ but $\eta_{0}(\alpha) \neq \eta_{1}(\alpha)$, without loss of generality $\eta_{\ell}(\alpha)=\ell$ and by clause $(v)$ above we get a contradiction.

2.8 Claim. 1) Assume $(*)_{\lambda}^{2}$ or just

$(*)_{\lambda}^{2^{-}} \mathfrak{K}$ has amalgamation in $\lambda$ and $L S(\mathfrak{K}) \leq \lambda$.

If $M_{0} \leq_{\mathfrak{K}} N_{0} \in K_{\lambda^{+}}$and $\left(M_{0}, M_{1}, a\right) \in K_{\lambda}^{3}$ then there is $N \in K_{\leq \lambda^{+}}$such that: $N_{0} \leq_{\mathfrak{K}} N$ and for every $c \in N$ satisfying $\operatorname{tp}\left(c, M_{0}, N\right)=\operatorname{tp}\left(a, M_{0}, M_{1}\right)$, there is a $\leq_{\mathfrak{K}^{-}}$embedding $h$ of $M_{1}$ into $N$ extending $i d_{M_{0}}$ such that $h(a)=c$ and $N \notin K_{\lambda^{+}} \Rightarrow$ $N$ is a $<_{\mathfrak{K}}$-maximal member of $K_{\lambda}$.

2) Assume $M_{0} \leq_{\mathfrak{K}} N_{0} \in K_{\lambda}$ and $\left(M_{0}, M_{1}, a\right) \in K_{\lambda}^{3}$ has the weak extension property. Then there is $N \in K_{\lambda^{+}}$such that: $N_{0} \leq_{\mathfrak{K}} N$ and for every $c \in N^{\prime}$ either for some $N^{\prime} \in K_{\lambda}$ we have $N_{0} \cup\{c\} \subseteq N \leq_{\mathfrak{K}} N$ and c does not strongly realize $\operatorname{tp}\left(a, M_{0}, M_{1}\right)$ or there is an $\leq_{\mathfrak{K}}$-embedding $h$ of $M_{1}$ into $N$ extending $i d_{M_{0}}$ such that $h(a)=c$.

Proof. 1) We choose by induction on $\alpha<\lambda^{+}$, a model $N_{\alpha} \in K_{\lambda}$ increasing (by $\leq_{\mathfrak{K}}$ ) continuous such that: for $\alpha$ even $N_{\alpha} \neq N_{\alpha+1}$ if $N_{\alpha}$ is not $\leq_{\mathfrak{K}}$-maximal, and for $\alpha$ odd let $\beta_{\alpha}=\operatorname{Min}\left\{\beta: \beta=\alpha+1\right.$ or $\beta \leq \alpha$ and there is $c \in N_{\beta}$ such that there is no $\leq_{K_{K}}$-embedding $h$ of $M_{1}$ into $N_{\alpha}$ extending $\operatorname{id}_{M_{0}}$ such that $h(a)=c$ but for some $N \in K_{\lambda}, N_{\alpha} \leq_{\mathfrak{K}} N$ and there is a $\leq_{\mathfrak{K}}$-embedding $h$ of $M_{1}$ into $N$ extending $\operatorname{id}_{M_{0}}$ such that $\left.h(a)=c\right\}$, and if $\beta_{\alpha} \leq \alpha$ then choose $N$ exemplifying this and let $N_{\alpha+1}=N$. By the definition of type we are done.

2) Same proof, note that the non $\leq_{\mathfrak{K}}$-maximality of $N_{\alpha}$ (and hence $N$ ) follows by a weak extension property.

2.9 Claim. Assume $(*)_{\lambda}^{2}$ or just:

$(*)_{\lambda}^{2^{-}} \mathfrak{K}$ has amalgamation for $\lambda$ and $L S(\mathfrak{K}) \leq \lambda$.

1) Assume that above $\left(M_{0}, M_{1}, a\right) \in K_{\lambda}^{3}$ there is no minimal member of $K_{\lambda}^{3} \underline{\text { then }}$ $\left(M_{0}, M_{1}, a\right)$ itself has the extension property.

2) If $\left(M_{0}, M_{1}, a\right) \in K_{\lambda}^{3}, M_{0} \leq_{\mathfrak{K}} N \in K$ and the number of $c \in N$ such that $\operatorname{tp}\left(c, M_{0}, N\right)=\operatorname{tp}\left(a, M_{0}, M_{1}\right)$ is $>\lambda$ then $\left(M_{0}, M_{1}, a\right)$ has the extension property.

3) Assume above $\left(M_{0}, M_{1}, a\right) \in K_{\lambda}^{3}$ there is no minimal member of $K_{\lambda}^{3} \underline{\text { then }}$

(*) for some $N$ we have: $M_{0} \leq_{\mathfrak{K}} N$ and $N$ is as required in part (2). 
2.10 Remark. 1) See 3.25.

2) Note that $(*)_{\lambda}^{2}$ is from 2.2 and $(*)_{\lambda}^{2} \Rightarrow(*)_{\lambda}^{2-}$ by 2.2 .

Proof. 1) Follows by part (2) and (3) (part (b)).

2) Without loss of generality $N$ has cardinality $\lambda^{+}$and also is as in 2.8 .

By 0.20(2) for any $M_{0}^{\prime}$ such that $M_{0} \leq_{\mathfrak{K}} M_{0}^{\prime} \in K_{\lambda}$ there is $N_{1}, N \leq_{\mathfrak{K}} N_{1} \in K_{\lambda^{+}}$ and a $\leq_{\mathfrak{K}}$-embedding $h$ of $M_{0}^{\prime}$ into $N_{1}$ extending $\operatorname{id}_{M_{0}}$ with $h_{c}(a)=c$. Now some $c \in N \backslash h\left(M_{0}^{\prime}\right)$ realizes $\operatorname{tp}\left(a, M_{0}, M_{1}\right)$ and there is an embedding $h_{c}$ of $M_{1}$ into $N$ extending $\operatorname{id}_{M_{0}}$ such that $h_{c}(a)=c$. Lastly let $N_{1}^{\prime} \leq_{\mathfrak{K}} N_{1}$ be of cardinality $\lambda$ and include $\operatorname{Rang}\left(h_{c}\right) \cup \operatorname{Rang}(h)$ (send $a$ to $c$ via $h_{c}$ ).

3) We first prove

$(*)_{0}$ For some $M_{0}^{+}, M_{0} \leq_{\mathfrak{K}} M_{0}^{+} \in K_{\lambda}$ and $\operatorname{tp}\left(a, M_{0}, M_{1}\right)$ has $>\lambda$ extensions in $\mathscr{S}\left(M_{0}^{+}\right)\left(\right.$in fact $\left.\geq \min \left\{2^{\mu}: 2^{\mu}>\lambda\right\}\right)$.

Proof of (a). Let $M_{\eta}^{0}, M_{\eta}^{1}, a_{\eta}, h_{\eta, \nu}\left(\eta \in \lambda^{\lambda^{+}}>2\right.$ and $\left.\nu \unlhd \eta\right)$ be as in the proof of 2.7 (i.e. satisfy $(i)-(v i)$ there) and $M_{<>}^{0}=M^{0}, M_{<>}^{1}=M^{1}$. Let $\mu=\operatorname{Min}\left\{\mu: 2^{\mu}>\lambda\right\}$, so ${ }^{\mu>} 2$ has cardinality $\leq \lambda$ and $\mu \leq \lambda$. Let ${ }^{\mu>} 2=\left\{\eta_{\zeta}: \zeta<\zeta^{*}\right\}$ be such that $\eta_{\xi} \triangleleft \eta_{\zeta} \Rightarrow \xi<\zeta$ and so $\zeta^{*}<\lambda^{+}$and without loss of generality is a limit ordinal. Now we can choose by induction on $\zeta \leq \zeta^{*}$ a model $M_{\zeta}^{*} \in K_{\lambda}$ and, if $\zeta<\zeta^{*}$ also a function $g_{\eta_{\zeta}}$ such that:

$(\alpha) M_{\zeta}^{*}$ is $\leq_{\mathfrak{K}^{-} \text {-increasing continuous in } \zeta}$

( $\beta) M_{0}^{*}=M_{\langle\rangle}$

$(\gamma) g_{\eta_{\zeta}}$ is a $\leq_{\mathfrak{K}^{-}}$-embedding of $M_{\eta_{\zeta}}^{0}$ into $M_{\zeta+1}^{*}$

( $\delta)$ if $\eta_{\xi} \triangleleft \eta_{\zeta}$ then $g_{\eta_{\zeta}} \circ h_{\eta_{\zeta}, \eta_{\xi}}=g_{\eta_{\xi}}$

$(\varepsilon)$ if $\xi<\xi_{0}, \xi<\xi_{1}, \eta_{\xi_{0}}=\eta_{\xi} \wedge\langle 0\rangle, \eta_{\xi_{1}}=\eta_{\xi} \wedge\langle 1\rangle$ then $\left(M_{\xi_{0}}^{*}=M_{\xi_{1}}^{*}\right.$ and $) g_{\eta_{\xi_{0}}}=g_{\eta_{\xi_{1}}}$.

So for $\eta \in{ }^{\mu} 2$ we can find $g_{\eta}$, a $\leq_{\mathfrak{K}^{-}}$-embedding of $M_{\eta}^{0}$ into $M_{\zeta^{*}}^{*}$, such that $g_{\eta} \circ h_{\eta, \eta \uparrow \alpha}=g_{\eta \uparrow \alpha}$ for every $\alpha<\mu$. We also can let

$p_{\eta}^{0}=g_{\eta}\left[\operatorname{tp}\left(a_{\eta}, M_{\eta}^{0}, M_{\eta}^{1}\right)\right] \in \mathscr{S}\left(M_{\zeta^{*}}^{*}\left\lceil\operatorname{Rang}\left(g_{\eta}\right)\right)\right.$, and find $p_{\eta}$ such that $p_{\eta}^{0} \leq p_{\eta} \in$ $\mathscr{S}\left(M_{\zeta^{*}}^{*}\right)$ (possible as $\mathfrak{K}_{\lambda}$ has amalgamation by $2.2(1)$ if $(*)_{\lambda}^{2}$ holds and by $(*)_{\lambda}^{2-}$ otherwise).

For $\eta \in{ }^{\mu} 2$ and $\alpha \leq \mu$ let $N_{\eta\lceil\alpha}^{0}=M_{\zeta^{*}}^{*}\left\lceil\right.$ Rang $\left(g_{\eta \uparrow \alpha}\right)$. Clearly for $\eta \in{ }^{\mu \geq 2}, N_{\eta}^{0}$ is well defined; $\eta \triangleleft \nu \in \in^{\mu \geq 2} \Rightarrow N_{\eta}^{0} \leq_{\mathfrak{K}} N_{\nu}^{0}$; and $N_{\eta^{\wedge}\langle 0\rangle}^{0}=N_{\eta^{\wedge}\langle 1\rangle}^{0}$. Also letting for $\eta \in{ }^{\mu} 2$, and $\alpha \leq \mu$, the type $p_{\eta\lceil\alpha}^{0}$ be $p_{\eta}^{0}\left\lceil N_{\eta\lceil\alpha}^{0}\right.$ we have: $p_{\eta}^{0} \in \mathscr{S}\left(N_{\eta}^{0}\right)$ is well defined, $\eta \triangleleft \nu \in{ }^{\mu \geq} 2 \Rightarrow p_{\eta}^{0} \leq p_{\nu}^{0}$ and $p_{\eta^{\wedge}\langle 0\rangle}^{0} \neq p_{\eta^{\wedge}\langle 1\rangle}^{0}$. Hence for $\eta_{0} \neq \eta_{1}$ from ${ }^{\mu} 2$ we have $p_{\eta_{0}} \neq p_{\eta_{1}}$. So $\left|\mathscr{S}\left(M_{\zeta^{*}}^{*}\right)\right| \geq 2^{\mu}>\lambda$.

Proof of $(*)$. We choose by induction on $i<\lambda^{+}, N_{i} \in K_{\lambda}$ which is $\leq_{\mathfrak{K}^{-}}$-increasing continuous, $N_{0}=M_{0}^{+}\left(M_{0}^{+}\right.$is from $(*)_{0}$ above) and for each $i$ some $c_{i} \in N_{i+1}$ realizes over $N_{0}$ a (complete) extension of $p=\operatorname{tp}\left(a, M_{0}, M_{1}\right)$ not realized in $N_{i}$. There is such type by clause $(*)$ above and there is such an $N_{i+1}$ as $\mathfrak{K}$ has amalgamation in $\lambda$. Clearly $c_{i} \notin N_{i}$ and so $\bigcup_{i<\lambda^{+}} N_{i}$ is as required.

4) By using 0.20(2) repeatedly $\lambda^{+}$times. 
2.11 Claim. Assume $(*)_{\lambda^{+}}^{2^{-}}$(from 2.9; that is $\mathfrak{K}$ has amalgamation in $\lambda$ and $L S(\mathfrak{K}) \leq \lambda)$.

If $\left(M_{0}, M_{1}, a\right) \leq\left(M_{0}^{\prime}, M_{1}^{\prime}, a\right)$ are from $K_{\lambda}^{3}$, and the second has the extension property, then so does the first.

Proof. Use amalgamation over $M_{0}$ : if $M_{0} \leq_{\mathfrak{K}} N_{0} \in K_{\lambda}$ we can find $N_{0}^{\prime}$ such that $M_{0}^{\prime} \leq_{\mathfrak{K}} N_{0}^{\prime} \in K_{\lambda}$ and there is a $\leq_{\mathfrak{K}}$-embedding of $N_{0}$ into $N_{0}^{\prime}$ over $M_{0}$. Now use " $\left(M_{0}^{\prime}, M_{1}^{\prime}, a\right)$ has the extension property" for $N_{0}^{\prime}$.

Now we introduce

2.12 Definition. For any models $M, M_{0} \in K_{\lambda}$, any type $p \in \mathscr{S}\left(M_{0}\right)$ and $f_{0}: M_{0} \underset{\text { iso }}{\stackrel{\text { onto }}{\longrightarrow}} M$ we let $\mathscr{S}_{p}(M)=\mathscr{S}_{M}^{p}=\left\{f_{0}(f(p)): f \in \operatorname{AUT}\left(M_{0}\right)\right\}$. Note: $\mathscr{S}_{M}^{p}$ does not depend on $f_{0}$. If $K$ is categorical in $\lambda, \mathscr{S}_{p}(M)$ is well defined for every $M \in K_{\lambda}$. We write also $\mathscr{S}_{\operatorname{tp}\left(a, M_{0}, M_{1}\right)}(M)$ or $\mathscr{S}_{\left(M_{0}, M_{1}, a\right)}(M)$ when $\left(M_{0}, M_{1}, a\right) \in$ $K_{\lambda}^{3}$.

2.13 Claim. Assume $(*)_{\lambda}^{2^{-}}+(*)_{\lambda^{+}}^{2}+(*)_{\lambda}^{3}+2^{\lambda^{+}}<2^{\lambda^{++}}+K_{\lambda^{+3}}=\emptyset$. If $\left(M_{0}, M_{1}, a\right) \in K_{\lambda}^{3}$ is minimal then it has the extension property.

Remark. Instead of $2^{\lambda^{+}}<2^{\lambda^{++}}$, we can just demand the definable weak diamond.

Proof. Assume not. By the previous two claims (2.9(1), 2.11) we may assume that $\left(M_{0}, M_{1}, a\right)$ is minimal. As $\mathfrak{K}$ has amalgamation in $\lambda^{+}$by $2.2(1)$, there is $M^{*} \in K_{\lambda^{++}}$which is saturated above $\lambda^{+}$(as $\left.K_{\lambda^{+3}}=\emptyset\right)$ hence $M^{*}$ is saturated above $\lambda$ (by 1.10(3)). By 2.6(1) +2.11 , without loss of generality

$\bigotimes_{0}\left(M_{0}, M_{1}, a\right)$ is reduced.

Let $h: M_{0} \rightarrow M^{*} \in K_{\lambda^{+}}$be a $\leq_{\mathfrak{K}^{-}}$-embedding and let $p=\operatorname{tp}\left(a, M_{0}, M_{1}\right)$. If $h(p)$ is realized in $M^{*}$ by $\geq \lambda^{+}$elements we are done by $2.9(2)$. So assume

$\bigotimes_{1} h(p)$ is realized by $\leq \lambda$ members of $M^{*}$.

Similarly

$\bigotimes_{1}^{+} g$ is realized by $\leq \lambda$ members of $M^{*}$ for $q=g\left(\operatorname{tp}\left(a, M_{0}^{\prime}, M_{1}^{\prime}\right)\right)$ if $g$ is a $\leq_{\mathfrak{K}^{-}}$-embedding of $M_{0}^{\prime}$ into $M^{*}$ and $\left(M_{0}^{\prime}, M_{1}^{\prime}, a\right) \geq\left(M_{0}, M_{1}, a\right)$.

Next we prove

$\bigotimes_{2}$ for some reduced $\left(M_{0}^{\prime}, M_{1}^{\prime}, a\right) \geq\left(M_{0}, M_{1}, a\right)$ from $K_{\lambda}^{3}$ we have $\left|\mathscr{S}_{\left(M_{0}^{\prime}, M_{1}^{\prime}, a\right)}\left(M_{0}^{\prime}\right)\right|>\lambda^{+}$.

Proof of $\bigotimes_{2}$. If not, we build two non-isomorphic members of $K_{\lambda^{+}}$as follows.

First: Choose by induction on $i<\lambda^{+},\left(N_{0, i}, N_{1, i}, a\right) \in K_{\lambda}^{3}$ reduced (see 2.6(1)), increasing continuously (see 2.6(2)), with $N_{0, i} \neq N_{0, i+1},\left(N_{0,0}, N_{1,0}, a\right)=\left(M_{0}, M_{1}, a\right)$; 
this is possible as $\left(N_{0, i}, N_{1, i}, a\right) \in K_{\lambda}^{3}$ has the weak extension property (by 2.4 see $2.3(1))$. Let $N^{1}=\bigcup_{i<\lambda^{+}} N_{0, i}$.

Second: Choose by induction on $i<\lambda^{+}, N_{i}^{0} \leq_{\mathfrak{K}} M^{*},\left\|N_{i}^{0}\right\|=\lambda, N_{i}^{0}$ strictly increasing continuous such that:

(*) for every $\beta<\lambda^{+}$, and $q \in \mathscr{S}_{\left(M_{0}^{\prime}, M_{1}^{\prime}, a\right)}\left(N_{\beta}^{0}\right)$ for some $\gamma \in\left(\beta, \lambda^{+}\right)$there are no $N^{\prime}, N_{\gamma}^{0} \leq_{\mathfrak{K}} N^{\prime} \in K_{\lambda}$ and $c \in N^{\prime} \backslash N_{\gamma}^{0}$ such that $c$ realize $q$.

This is straightforward by $\otimes_{1}+\otimes_{2}$ and bookkeeping. Let $N^{0}=\bigcup_{i<\lambda^{+}} N_{i}^{0}$. By categoricity of $K_{\lambda^{+}}$there is an isomorphism $g$ from $N^{1}$ onto $N^{0}$, so $E=\left\{\delta<\lambda^{+}: g\right.$ maps $N_{0, \delta}$ onto $\left.N_{\delta}^{0}\right\}$ is a club of $\lambda^{+}$. Now let $\delta^{*} \in E$, and apply $(*)$ for $\beta=\delta^{*}, q=g\left(\operatorname{tp}\left(a, N_{0, \delta^{*}}, N_{1, \delta^{*}}\right)\right)$ to get $\gamma$. Choose $\delta \in E$ which is $>\gamma$. Now $N_{1, \delta}$ gives a contradiction.

Without loss of generality

$\bigotimes_{3}\left|\mathscr{S}_{\left(M_{0}, M_{1}, a\right)}\left(M_{0}\right)\right|>\lambda^{+}$and $p=\operatorname{tp}\left(a, M_{0}, M_{1}\right)$.

Next we claim

$\bigotimes_{4}$ If $M \in K_{\lambda}, M \leq_{\mathfrak{K}} M^{*}, \Gamma \subseteq \bigcup\left\{\mathscr{S}_{p}\left(M^{\prime}\right): M^{\prime} \leq_{\mathfrak{K}} M,\left\|M^{\prime}\right\|=\lambda\right\}$, $|\Gamma| \leq \lambda^{+}$, then

$$
\begin{gathered}
\Gamma^{*}=:\left\{q \in \mathscr{S}_{p}(M): \text { there is } M^{\prime}, M \leq_{\mathfrak{K}} M^{\prime},\left\|M^{\prime}\right\|=\lambda,\right. \\
\left.M^{\prime} \text { realizes } q \text { but no } r \in \Gamma\right\}
\end{gathered}
$$

has cardinality $\lambda^{++}$, in fact $\left|\mathscr{S}_{p}(M) \backslash \Gamma^{*}\right| \leq \lambda^{+}$.

Proof of $\otimes_{4}$. Without loss of generality $\left|M^{*}\right|=\lambda^{++}$(i.e. the universe of $M^{*}$ is $\left.\lambda^{++}\right)$. For every $q \in \mathscr{S}_{p}(M)$ there is a triple $\left(M_{0}, M_{1, q}, a_{q}\right)$ isomorphic to $\left(M_{0}, M_{1}, a\right)$ (hence reduced) such that $\operatorname{tp}\left(a_{q}, M_{0}, M_{1, q}\right)=q$. As $M^{*}$ is saturated above $\lambda$, by 0.26 without loss of generality $M_{1, q} \leq_{\mathfrak{K}} M^{*}$. Now

Without loss of generality $\delta<\lambda^{++} \&\left(\lambda^{+}\right.$divides $\left.\delta\right) \Rightarrow M_{\delta}=: M^{*}\left\lceil\delta \leq_{\mathfrak{K}} M^{*}\right.$.

$(*)_{0} \quad q_{1} \neq q_{2} \Rightarrow a_{q_{1}} \neq q_{q_{2}}$ and

$(*)_{1} \quad a_{q} \notin \delta \& \delta<\lambda^{++} \& \lambda^{+}$divides $\delta \Rightarrow M_{1, q} \cap M_{\delta}=M$.

[Why? As $\left(M, M_{1, q}, a_{q}\right)$ is reduced].

Now if $r \in \Gamma$, say $r \in \mathscr{S}_{p}\left(M^{\prime \prime}\right)$ then by $\otimes_{1}$ we know $A_{r}=\left\{c \in M^{*}: c\right.$ realizes $\left.r\right\}$ has cardinality $\leq \lambda$ and hence $A=\bigcup\left\{A_{r}: r \in \Gamma\right\}$ has cardinality $\leq \lambda^{+}$, so we can find $\delta<\lambda^{++}$divisible by $\lambda^{+}$such that $A \subseteq \delta$. So as (by $\otimes_{3}$ ) we have $\left|\mathscr{S}_{p}(M)\right|>$ $\lambda^{+}$hence we can find $q[\delta] \in \mathscr{S}_{p}(M)$ such that $a_{q(\delta)} \notin \delta$ hence $\left(M, M_{1, q[\delta]}, a_{q[\delta]}\right)$, exemplifies the conclusion of $\otimes_{4}$.

Final contradiction: By $\otimes_{4}$ we can construct $2^{\lambda^{+}}$non-isomorphic members of $K_{\lambda^{+}}$ using 1.6(1) as follows. We choose by induction on $\alpha<\lambda^{+}$, for every $\eta \in$ ${ }^{\alpha} 2, M_{\eta}, p_{\eta}^{0}, p_{\eta}^{1}$ such that: 
(a) $M_{<>}=M_{0}$

(b) $M_{\eta} \in K_{\lambda}$

(c) $\left\langle M_{\eta \uparrow \beta}: \beta \leq \ell g(\eta)\right\rangle$ is $<_{\mathfrak{K}}$-increasing continuous

(d) $p_{\eta} \in \mathscr{S}_{p}\left(M_{\eta \uparrow \beta}\right)$

(e) for $\beta \leq \alpha$, we have $p_{\eta}^{0}, p_{\eta}^{1} \in \mathscr{S}\left(M_{\eta}\right)$ and: $M_{\eta}$ realizes $p_{\eta\lceil\beta}^{\ell}$ iff $\beta<\alpha \& \ell=$ $\eta(\beta)$.

If $\alpha=0$ or $\alpha$ is a limit, there is no problem to define $M_{\eta}$ for $\eta \in{ }^{\alpha} 2$. If $M_{\eta}$ is defined, we can choose by induction on $i<\lambda^{++},\left(N_{\eta, i}, a_{\eta, i}\right)$ such that $\left(M_{\eta}, N_{\eta, i}, a_{\eta, i}\right) \in K_{\lambda}^{3}$, $\operatorname{tp}\left(a_{\eta, i}, M_{\eta}, N_{\eta, i}\right) \in \mathscr{S}_{p}\left(M_{\eta}\right)$ and $N_{\eta, i}$ omits any $q \in\left\{p_{\eta\lceil\beta}^{\ell}: \beta<\ell g(\eta), \ell \neq \eta(\beta)\right\} \cup\left\{\operatorname{tp}\left(b, M_{\eta}, N_{\eta, j}\right): j<i\right.$ and $b \in N_{\eta, j}$ and $\left.\operatorname{tp}\left(b, M_{\eta}, N_{\eta, j}\right) \in \mathscr{S}_{p}\left(M_{\eta}\right)\right\}$. By $\otimes_{4}$ we can choose $\left(N_{\eta, j}, a_{\eta, j}\right)$.

Hence $\left|W_{\eta, i}\right| \leq \lambda$ where $W_{\eta, i}=\left\{j<\lambda^{++}\right.$: for some $b \in N_{\eta, i}$ we have $\operatorname{tp}\left(b, M_{\eta}, N_{\eta, i}\right)=$ $\left.\operatorname{tp}\left(a_{\eta, j}, M_{\eta}, N_{\eta, j}\right)\right\}$.

Hence we can find $i<j<\lambda^{++}$such that $i \notin W_{\eta, j} \quad \& \quad j \notin W_{\eta, i}$. Let $M_{\eta^{\wedge}\langle 0\rangle}=N_{\eta, i}, M_{\eta^{\wedge}\langle 1\rangle}=N_{\eta, j}, p_{\eta}^{0}=\operatorname{tp}\left(a_{\eta, i}, M_{\eta}, N_{\eta, i}\right), p_{\eta}^{1}=\operatorname{tp}\left(a_{\eta, j}, M_{\eta}, N_{\eta, j}\right)$.

Let for $\eta \in{\lambda^{+}}^{+} 2, M_{\eta}=\bigcup_{\alpha<\lambda^{+}} M_{\eta\lceil\alpha}$, and apply 1.6(1).

2.14 Conclusion. $\left[(*)_{\lambda}^{2^{-}}+(*)_{\lambda^{+}}^{2}+(*)_{\lambda}^{3}+2^{\lambda^{+}}<2^{\lambda^{++}}+K_{\lambda^{+3}}=\emptyset\right]$.

Every $\left(M_{0}, M_{1}, a\right) \in K_{\lambda}^{3}$ has the extension property.

Proof. By 2.11 and $2.9+2.12$.

2.15 Remark. Conclusion 2.14 says in other words:

if

(a) $L S(\mathfrak{K}) \leq \lambda$

(b) $K$ is categorical in $\lambda$ and in $\lambda^{+}$

(c) $1 \leq I\left(\lambda^{++}, K\right)<2^{\lambda^{++}}$

(d) $K_{\lambda^{+3}}$ is empty

(e) $2^{\lambda^{+}}<2^{\lambda^{++}}$(or just definable weak diamond)

then every triple $\left(M_{0}, M_{1}, a\right)$ in $K_{\lambda}^{3}$ has the extension property.

2.16 Claim. $\left[(*)_{\lambda}^{3}\right.$, in other words $L S(\mathfrak{K}) \leq \lambda ; \mathfrak{K}$ categorical in $\lambda$ and in $\lambda^{+}$; and $\left.1 \leq I\left(\lambda^{++}, K\right)<2^{\lambda^{++}}\right]$.

If $M_{0} \leq_{\mathfrak{K}} M_{1}$ are in $K_{\lambda}$ then we can find $\alpha<\lambda^{+}$and $\left\langle N_{i}: i \leq \alpha\right\rangle$ which is $\leq_{\mathfrak{K}}$-increasing continuous, $N_{i} \in K_{\lambda},\left(N_{i}, N_{i+1}, a_{i}\right) \in K_{\lambda}^{3}$ is reduced, $M_{0}=N_{0}$, and $M_{1} \leq_{\mathfrak{K}} N_{\alpha}$.

Proof. If not, we can contradict categoricity in $K_{\lambda^{+}}$(similar to the proof of $\otimes_{2}$ during the proof of 2.12). 
Without loss of generality $M_{0} \neq M_{1}$. We choose by induction on $i<\lambda^{+}$, $N_{i}^{0} \in K_{\lambda}, \leq_{\mathfrak{K}^{-}}$-increasing continuous such that $\left(N_{i}^{0}, N_{i+1}^{0}\right) \cong\left(M_{0}, M_{1}\right)$ (possible by 2.6(9) and the categoricity of $K$ in $\lambda$ ). Let $N^{0}=\bigcup_{i<\lambda^{+}} N_{i}^{0}$.

We choose by induction on $i<\lambda^{+}, N_{i}^{1} \in K_{\lambda}, \leq_{\mathfrak{K}^{-}}$-increasing continuous and $a_{i}$ such that $\left(N_{i}^{1}, N_{i+1}^{1}, a_{i}\right) \in K_{\lambda}^{3}$ is reduced and let $N^{1}=\bigcup_{i<\lambda^{+}} N_{i}^{1}$ (possible by 2.6(1) and the categoricity of $K$ in $\lambda$ ). So by the categoricity in $\lambda^{+}$without loss of generality $N^{1}=N^{0}$, hence for some $\delta_{1}<\delta_{2}<\lambda^{+}$we have

$$
N_{\delta_{1}}^{0}=N_{\delta_{1}}^{1}, N_{\delta_{2}}^{0}=N_{\delta_{2}}^{1} .
$$

By changing names $\left(N_{\delta_{1}}^{0}, N_{\delta_{1}+1}^{0}\right)=\left(M_{0}, M_{1}\right)$ and so $\left\langle N_{\delta_{2}+i}: i \leq \delta_{2}-\delta_{1}\right\rangle$ is as required.

2.17 Conclusion. $\left[(*)_{\lambda}^{2^{-}}+(*)_{\lambda^{+}}^{2}+(*)_{\lambda}^{3}+2^{\lambda^{+}}<2^{\lambda^{++}}+K_{\lambda+3}=\emptyset\right.$, i.e. the assumption of 2.14].

$K_{\lambda}$ has disjoint amalgamation $\left(M_{2}, M_{1}\right.$ are in disjoint amalgamation over $M_{0}$ in $M_{3}$ if $\left.M_{0} \leq_{\mathfrak{K}} M_{\ell} \leq_{\mathfrak{K}} M_{3}, M_{1} \cap M_{2}=M_{0}\right)$.

Proof. By 2.16 and iterated applications of 2.14 . 


\section{$\S 3$ Non-STRUCTURE}

The first major aim of this section is to prove the density of minimal types using as set theoretic assumptions only $2^{\lambda}<2^{\lambda^{+}}<2^{\lambda^{++}}$from cardinal arithmetic. The second aim is to prepare for a proof of a weak form of uniqueness of amalgamation in $\mathfrak{K}_{\lambda}$. Our aim is also to explain various methods. The proofs are similar to the ones in $[$ Sh $87 \mathrm{~b}, \S 6]$.

The immediate role of this section is to get many models in $\lambda^{++}$from the assumption "the minimal triples in $K_{\lambda}^{3}$ are not dense": in 3.25 we get this under some additional assumptions, and in 3.28 we get it using only the additional assumption $I\left(\lambda, K^{+3}\right)=0$, which suffices for our main theorem (this does not suffice for the theorem of [Sh 600], hence is eliminated there).

But the section is done in a more general fashion, so let us first explain two general results concerning the construction of many models based on repeated "failures of amalgamation" or "nonminimality of types".

In 3.19, we give a construction assuming the ideal of small subsets of $\lambda^{+}$(that is WDmId $\left.\left(\lambda^{+}\right)\right)$is not $\lambda^{++}$-saturated, as exemplified by $\left\langle S_{\alpha}: \alpha<\lambda^{++}\right\rangle$. We build for $\eta \in{ }^{\left(\lambda^{+2}\right)>} 2$ models $M_{\eta} \in K_{\lambda^{+}}$such that $M_{\eta}=\bigcup_{\alpha<\lambda^{+}} M_{\eta, \alpha},\left|M_{\eta}\right|=\lambda \times(1+\ell g \eta)$ and $\nu \triangleleft \eta \Rightarrow M_{\nu} \leq_{\mathfrak{K}} M_{\eta}$. Building $M_{\eta^{\wedge}\langle\ell\rangle}$ manufacture $M_{\eta^{\wedge}\langle\ell\rangle, \alpha+1}$ as a limit of models $\left\langle M_{\eta^{\wedge}\langle\ell\rangle, \alpha}: \alpha<\lambda^{+}\right\rangle$, a representation of $M_{\eta^{\wedge}\langle\ell\rangle}$, usually in a way predetermined simply, except when $\alpha \in S_{\ell g(\eta)}$ and $\ell=1$, and then we consult a weak diamond sequence. This is like 1.6(1), but there we use our understanding of models in $K_{\lambda}$ to build many models in $K_{\lambda^{+}}$while here we build models in $K_{\lambda^{++}}$, thus getting $2^{\lambda^{+2}}$ models in $\lambda^{+2}$. We even get $2^{\left(\lambda^{+2}\right)}$ models in $K_{\lambda^{+2}}$ with none $\leq_{\mathfrak{K}}$-embeddable into any other.

A second proof 3.23 is like 1.4(1) in the sense that we get only close to $2^{\lambda^{++}}$ models. It is similar to [Sh 87b, 6.4], and the parallel to [Sh 87b, 6.3] holds here. So we have to find an analog of [Sh $87 \mathrm{~b}$, definition $6.5,6.7]$. But there we use fullness on the side (meaning: $M \in K_{\lambda}$ is full over $N \in K_{\lambda}$ if $N \leq_{\mathfrak{K}} M$, and $(M, c)_{c \in N}$ is saturated), but we do not have this yet.

We still have not explained the framework of this section. In $3.1-3.5$ we present construction frameworks $\mathbf{C}$, which involve sequences of models of length $\leq \lambda$ each of cardinality $<\lambda$ and in particular, define local and nice $\mathbf{C}$. In our applications here $\lambda^{+}$plays the role of $\lambda$ (and $<\lambda^{+}$is specialized to $\lambda$ ).

Then in $3.6-3.8$ we present examples of such frameworks. Our intention is to use the limit of a sequence $\left\langle M_{\alpha}: \alpha<\lambda\right\rangle$ as an approximation to a model of cardinality $\lambda^{+}$. For this we define in 3.10 - 3.11 a successor relation (next approximation), modulo a " $<\lambda$-amalgamation choice function"; this is denoted $\bar{M}^{1} \leq_{F_{1}}^{\text {at }} \bar{M}^{2}$. Iterating it we get the quasi order $\leq_{F}$ (see 3.13). In 3.14 we define the key coding properties, (of an amalgamation choice function $F$ for the framework $\mathbf{C}$ ). The intention is that these coding properties suffice to build many non-isomorphic models in $\lambda^{+}$. In 3.17 we give the "atomic step" for this construction.

In 3.18 we prove the existence of $2^{\lambda^{+}}$non-isomorphic models, using the $\lambda$-coding property. As we do not have this in some applications we have in mind, we next turn to the weak $\lambda$-coding property in 3.19 as well as the weak (local) $\lambda$-coding property and corresponding properties of $F$ (all in Definition 3.20, 3.22), connect 
them 3.21 , and prove there are many models in 3.23 .

Lastly, 3.25, 3.28 deal with our concrete case: if the minimal triples in $\mathfrak{K}_{\lambda}^{3}$ are not dense then in most cases failures of amalgamation lead to the $\lambda^{+}$-coding property and hence to many models in cardinality $\lambda^{++}$.

Note generally that we mainly axiomatize the construction of models in $\lambda^{+}$, not how we get $\bar{M}^{\prime}, \bar{M} \leq_{F, a}^{\text {at }} \bar{M}^{\prime} \in \mathbf{S} e q_{\lambda}$ that is coding properties; for the last point, see the examples just cited.

Later, in 6.10, we shall need again to use the machinery from this section, in trying to prove that there are enough cases of disjoint amalgamation in $\mathfrak{K}_{\lambda}$.

We may want to turn the framework presented here into a more general one; see more in [Sh 600].

3.1 Context.

1) $\mathfrak{K}$ is an abstract elementary class.

2) But $=_{M}$ or $=_{\mathfrak{K}}$ is just an equivalence relation, i.e. for $M \in K,={ }^{M}$ is an equivalence relation on $|M|$, moreover a congruence relation relative to all relations and functions in $\tau(M)$ that is for $R \in \tau(M)$ an $n$-ary relation, we have

$$
\left.\bigwedge_{\ell<n} a_{i}={ }^{M} b_{i} \Rightarrow\left\langle a_{0}, \ldots, a_{n-1}\right\rangle \in R^{M} \equiv\left\langle b_{0}, \ldots, b_{n-1}\right\rangle \in R^{M}\right) .
$$

We let $\|M\|=\left|(M) /={ }^{M}\right|$ and

$$
K_{\lambda, \mu}=\left\{M:|M| \text { has } \mu \text { elements and }|M| /={ }^{M} \text { has } \lambda \text { elements }\right\} .
$$

3) Now the meaning of $\leq_{\mathfrak{K}}$ should be clear but $M<_{\mathfrak{K}} N$ means $(M \in K, N \in K$ and) $M \subseteq N$ and $M /={ }^{M} \leq_{\mathfrak{K}} N /={ }^{N}$ and $\exists a \in N\left[a /={ }^{N} \notin\left(M /={ }^{N}\right)\right]$ i.e. $(\exists a \in$ $N)(\forall b \in M)\left(\neg a={ }^{N} b\right)$.

4) $K_{\lambda}^{3}=\left\{(M, N, a): M \leq_{\mathfrak{K}} N\right.$ are from $K_{\lambda, \lambda}$ and $\left.a \in N,\left(a /={ }^{N}\right) \notin\left(M /={ }^{N}\right)\right\}$.

5) In this content " $R$ is an isomorphism relation from $M_{1}$ onto $M_{2}$ if

(a) $R \subseteq M_{1} \times M_{2}$

(b) $a_{1}={ }^{M_{1}} b_{1} \& a_{2}={ }^{M_{2}} b_{2} \Rightarrow a_{1} R a_{2} \leftrightarrow b_{1} R b_{2}$

(c) $\left(\forall x \in M_{1}\right)\left(\exists y \in M_{2}\right) x R y$

(d) $\left(\forall y \in M_{2}\right)\left(\exists x \in M_{1}\right) x R y$

(e) if $Q \in \tau\left(M_{1}\right)=\tau\left(M_{2}\right)$ is an $n$-place relation and $a_{\ell} Q b_{\ell}$ for $\ell=0, \ldots, n-1$ then $\left(a_{0}, \ldots, a_{n-1}\right) \in Q^{M_{1}} \rightarrow\left(b_{0}, \ldots, b_{n-1}\right) \in Q^{M_{2}}$.

3.2 Explanation. The need of 3.1(2) is just to deal with amalgamations which are not necessarily disjoint. If we use disjoint amalgamation, we can omit 3.1(2) below on Definition 3.10, $a$ disappears so $F$ is four place and use $K_{\lambda}$ instead of $K_{\lambda, \lambda}$. This is continued in [Sh 600, 2.17]. May be better understood after reading 3.10, after clause (c). 
3.3 Definition. Let $\lambda$ be regular uncountable and $\mathfrak{K}$ an abstract elementary class. 1) A $\lambda$-construction framework $\mathbf{C}=\mathbf{C}\left(\mathfrak{K}^{+}, \mathbf{S} e q, \leq^{*}\right)$ means (we shall use it below with $\lambda^{+}$playing the role of $\lambda$ ):

(a) $\tau^{+}=\tau^{+}\left(\mathfrak{K}^{+}\right)$is a vocabulary extending $\tau$. $\mathfrak{K}^{+}$is an abstract elementary class satisfying axioms I,II,III from 0.6 and $M \leq_{\mathfrak{K}^{+}} N \Rightarrow M \uparrow \tau \leq_{\mathfrak{K}} N \mid \tau$. Furthermore $\mathfrak{K}^{+}=\mathfrak{K}_{<\lambda}^{+}$. As above, equality (in $\tau$ ) is a congruence relation.

(b) $\mathbf{S} e q=\bigcup_{\alpha \leq \lambda} \mathbf{S} e q_{\alpha}$ where for $\alpha \leq \lambda, \mathbf{S} e q_{\alpha}$ is a subset of $\left\{\bar{M}: \bar{M}=\left\langle M_{i}: i<\alpha\right\rangle, M_{i} \in \mathfrak{K}^{+}\right.$is $\leq_{\mathfrak{K}^{+}}$-increasing continuous $\}$.

For $\alpha=\lambda$ we require further that $M /={ }^{M}$ has cardinality $\lambda$, where $M=\bigcup_{i<\lambda} M_{i}$.

(c) $\leq^{*}$ is a relation on triples $x, y, t$ written $x \leq_{t}^{*} y$ for $x, y \in \mathbf{S} e q$ and $t$ a set of pairwise disjoint closed intervals of $\ell g(x)$.

We require:

(d) $\mathbf{S} e q$ is closed under isomorphism and initial segments.

(e) If $\bar{M}^{1} \leq_{t}^{*} \bar{M}^{2}$ and $\gamma \in \cup t$ then $M_{\gamma}^{1} \leq_{\mathfrak{K}^{+}} M_{\gamma}^{2}$ and hence $M_{\gamma}^{1} \uparrow \tau \leq_{\mathfrak{K}} M_{\gamma}^{2} \uparrow \tau$.

(f) If $\bar{M}^{1} \leq_{t}^{*} \bar{M}^{2}, s \subseteq t$, and $\bar{M}^{2} \unlhd \bar{M}^{3} \in \mathbf{S} e q$ then $\bar{M}^{1} \leq_{s}^{*} \bar{M}^{3}$.

(g) If $t$ is a set of closed pairwise disjoint intervals of $\ell g(\bar{M})$ and $\bar{M} \in \mathbf{S} e q$ then $\bar{M} \leq_{t}^{*} \bar{M}$.

\subsection{Convention/Definition:}

1) From now on $\mathbf{C}$ will be a $\lambda$-construction framework.

2) If $\bar{M} \in \mathbf{S} e q_{\lambda}$ then we let $\bar{M}=\left\langle M_{i}: i<\lambda\right\rangle$ and $M=: \bigcup_{i<\lambda} M_{i}$; similarly with $\bar{M}^{x}=\left\langle M_{i}^{x}: i<\lambda\right\rangle$.

3) $K_{\lambda}^{\mathrm{qr}}=\left\{(\bar{M}, \mathbf{f}): \bar{M} \in \mathbf{S} e q_{\lambda}\right.$ and $\left.\mathbf{f}: \lambda \rightarrow \lambda\right\}$.

4) If $\left(\bar{M}^{\ell}, \mathbf{f}^{\ell}\right) \in K_{\lambda}^{\mathrm{qr}}$ for $\ell=1,2$ then $\left(\bar{M}^{1}, \mathbf{f}^{1}\right) \leq\left(\bar{M}^{2}, \mathbf{f}^{2}\right)$ means that: for some club $E$ of $\lambda$, we have

(a) $\delta \in E \Rightarrow \mathbf{f}^{1}(\delta) \leq \mathbf{f}^{2}(\delta)$ and

(b) $\bar{M}^{1} \leq_{t}^{*} \bar{M}^{2}$ where $t=t_{E, \mathbf{f}^{1}}=\left\{\left[\delta, \delta+\mathbf{f}^{1}(\delta)\right]: \delta \in E\right\}$.

5) $\mathbf{S} e q^{s}=\left\{\bar{M} \in \mathbf{S} e q: \bigcup_{i}\left|M_{i}\right|\right.$ is a set of ordinals $\left.<\lambda^{+}\right\}$; similarly for $\mathbf{S} e q_{\alpha}$.

6) $K_{\lambda}^{\mathrm{qs}}=\left\{(\bar{M}, \mathbf{f}) \in K_{\lambda}^{\mathrm{qr}}: \bar{M} \in \mathbf{S} e q_{\lambda}^{s}\right\}$.

7) $\mathbf{C}$ is local (respectively, revised local) if (a-c) following hold:

(a) $\bar{M}=\left\langle M_{i}: i<\alpha\right\rangle \in \mathbf{S} e q_{\alpha}$ iff:

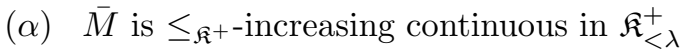

( $\beta) ~ i+1<\alpha \Rightarrow\left\langle M_{i}, M_{i+1}\right\rangle \in \mathbf{S} e q_{2}$

$(\gamma)$ if $\alpha=\lambda$ then $|M|={ }^{M} \mid=\lambda$, (recall $M=\bigcup_{i<\lambda} M_{i}$ ) 
(b) for $\bar{M}^{1}, \bar{M}^{2} \in \mathbf{S} e q$ and $t$ a set of pairwise disjoint closed intervals contained in $\ell g\left(\bar{M}^{1}\right)$ we have:

$$
\bar{M}^{1} \leq_{t}^{*} \bar{M}^{2} \underline{\text { iff }}\left[\gamma_{1}, \gamma_{2}\right] \in t \text { implies }
$$

( $\alpha) \quad \gamma \in\left[\gamma_{1}, \gamma_{2}\right] \Rightarrow M_{\gamma}^{1} \leq_{\mathfrak{K}^{+}} \bar{M}_{\gamma}^{2}$

$(\beta)$ in the local case: $\gamma \in\left[\gamma_{1}, \gamma_{2}\right) \Rightarrow\left\langle M_{\gamma}^{1}, M_{\gamma+1}^{1}\right\rangle \leq_{\{[0,1]\}}^{*}\left\langle M_{\gamma}^{2}, M_{\gamma+1}^{2}\right\rangle$;

in the revised local case: if $\ell g\left(\bar{M}^{1}\right), \ell g\left(\bar{M}^{2}\right)<\lambda$ then

$\gamma \in\left[\gamma_{1}, \gamma_{2}\right] \Rightarrow\left\langle M_{\gamma}^{1}, M^{1}\right\rangle \leq_{\{[0,1]\}}^{*}\left\langle M_{\gamma}^{2}, M^{2}\right\rangle$, and generally for some club $E$ of $\lambda, \gamma \in\left[\gamma_{1}, \gamma_{2}\right] \quad \& \quad \gamma<\delta \in E \Rightarrow\left\langle M_{\gamma}^{1}, \bigcup_{\beta<\delta} M_{\beta}^{1}\right\rangle \leq_{\{[0,1]\}}^{*}$ $\left\langle M_{\gamma}^{2}, \bigcup_{\beta<\delta} M_{\beta}^{2}\right\rangle$ (and if $\ell g\left(\bar{M}^{\ell}\right)=\alpha_{\ell}<\delta, \bigcup_{\beta<\delta} M_{\beta}^{\ell}$ means $\left.\bigcup_{\beta<\alpha^{\ell}} M_{\beta}^{\ell}\right)$.

(c) if $\left\langle M_{0}^{\zeta}, M_{1}^{\zeta}\right\rangle \in \mathbf{S} e q_{2}$ for $\zeta<\zeta^{*}<\lambda,\left\langle M_{0}^{\zeta}: \zeta \leq \zeta^{*}\right\rangle$ and $\left\langle M_{1}^{\zeta}: \zeta \leq \zeta^{*}\right\rangle$ are $\leq_{\mathfrak{K}^{+}}$-increasing continuous, and $\zeta<\zeta^{*} \Rightarrow\left\langle M_{0}^{\zeta}, M_{1}^{\zeta}\right\rangle \leq_{\{0,1]\}}^{*}\left\langle M_{0}^{\zeta+1}, M_{1}^{\zeta+1}\right\rangle$ then $\left\langle M_{0}^{0}, M_{1}^{0}\right\rangle \leq_{\{[0,1]\}}^{*}\left\langle M_{0}^{\zeta^{*}}, M_{1}^{\zeta^{*}}\right\rangle \in \mathbf{S e q}$.

So intervals $[\alpha, \alpha] \in t$ are irrelevant for the local version. In the revised local version it is natural to add monotonicity for $\leq_{\{[0,1]\}}$.

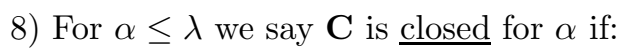

( $\alpha) \bar{M}=\left\langle M_{i}: i<\alpha\right\rangle \in \mathbf{S} e q \underline{\text { iff }} \beta<\alpha \Rightarrow \bar{M} \uparrow(\beta+1) \in \mathbf{S} e q$

( $\beta)$ if $\bar{M}^{\ell}=\left\langle M_{i}^{\ell}: i<\alpha_{\ell}\right\rangle \in \mathbf{S} e q$ for $\ell=1,2$ and $\alpha=\alpha_{1}<\alpha_{2}$, then

$$
\bar{M}^{1} \leq_{t}^{*} \bar{M}^{2} \Leftrightarrow \bar{M}^{1} \leq_{t}^{*} \bar{M}^{2} \uparrow \alpha .
$$

9) $\mathbf{C}$ is disjoint if: $\bar{M}^{1} \leq_{t}^{*} \bar{M}^{2},\left[\gamma_{1}, \gamma_{2}\right] \in t, \gamma \in\left[\gamma_{1}, \gamma_{2}\right)$ implies $M_{\gamma}^{1}=M_{\gamma+1}^{1} \cap M_{\gamma}^{2}$. C is truly disjoint if: $\bar{M}^{1} \leq_{t}^{*} \bar{M}^{2},\left[\gamma_{1}, \gamma_{2}\right] \in t, \gamma \in\left[\gamma_{1}, \gamma_{2}\right]$ implies $M_{\gamma}^{1}=M^{1} \cap M_{\gamma}^{2}$.

10) In $K_{\lambda}^{\mathrm{qr}}$, we say $(\bar{M}, \mathbf{f})$ is a $\underline{\text { m.u.b. }}$ (minimal upper bound) of $\left\langle\left(\bar{M}^{\xi}, \mathbf{f}^{\xi}\right): \xi<\delta\right\rangle$ if

(a) $\xi<\delta \Rightarrow\left(\bar{M}^{\xi}, \mathbf{f}^{\xi}\right) \leq(\bar{M}, \mathbf{f})$ and

(b) for any $\left(\bar{M}, \mathbf{f}^{\prime}\right)$ satisfying (a), for some club $E$ of $\lambda$ we have: if $\alpha \in E$ and $j \leq \mathbf{f}(\alpha)$ then $\mathbf{f}(\alpha) \leq \mathbf{f}^{\prime}(\alpha)$ and $M_{\alpha+j} \leq_{\mathfrak{K}^{+}} M_{\alpha+j}^{\prime}$.

When we require an increasing sequence in $K_{\lambda}^{\mathrm{qr}}$ to be continuous we mean that a m.u.b. is used at limits.

11) We say $\mathbf{C}$ is explicitly local if it is local and

$(d)$ if $\zeta^{*}<\lambda$ is a limit ordinal, $\left\langle M_{0}^{\zeta}, M_{1}^{\zeta}\right\rangle \in \mathbf{S} e q_{2}$ for $\zeta \leq \zeta^{*}$ and for $\ell=0,1$

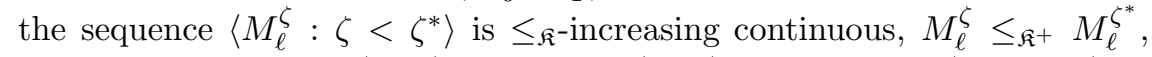
and $\zeta<\xi \leq \zeta^{*} \Rightarrow\left\langle M_{0}^{\zeta}, M_{1}^{\zeta}\right\rangle \leq_{\{[0,1]\}}^{*}\left\langle M_{0}^{\xi}, M_{1}^{\xi}\right\rangle$ then $\left\langle\bigcup_{\zeta<\zeta^{*}} M_{0}^{\zeta}, \bigcup_{\zeta<\zeta^{*}} M_{1}^{\zeta}\right\rangle$ is $\leq_{\{[0,1]\}}\left(M_{0}^{\zeta^{*}}, M_{1}^{\zeta^{*}}\right)$. 
12) $\mathbf{C}$ is closed if it is closed for every ordinal $\leq \lambda$.

13) $\mathbf{C}$ is semi (respectively almost) closed, as witnessed by $G$, if:

(a) $\mathbf{C}$ is closed for every limit ordinal $\delta<\lambda$;

(b) $G$ is a function from $\mathbf{S} e q_{<\lambda}$ to $\mathbf{S} e q_{<\lambda}$ such that $\bar{M} \triangleleft G(\bar{M}) ;$

(c) $\bar{M}=\left\langle M_{\alpha}: \alpha<\lambda\right\rangle$ belongs to $\mathbf{S} e q_{\lambda}$ if $\bar{M}$ obeys $G$, which means: $\beta<\lambda \Rightarrow$ $\bar{M}\left\lceil\beta \in \mathbf{S} e q_{\beta}\right.$ and $\{\alpha<\lambda: G(\bar{M}\lceil\alpha) \triangleleft \bar{M}\}$ is unbounded in $\lambda$

$(d)$ in the almost closed version, we add: $G(\bar{M})$ depends on $\bigcup \bar{M}$ only.

14) $\mathbf{C}$ is $\underline{\lambda \text {-nice if }}$

(a) $\leq$ is a transitive on $K_{\lambda}^{q r}$;

(b) any increasing continuous sequence in $K_{\lambda}^{q r}$ of length $<\lambda^{+}$has a m.u.b.; (see part (10)) (not necessarily unique)

(c) $\mathbf{C}$ is closed (see part (12)).

15) $\mathbf{C}$ is almost $\lambda$-nice (as witnessed by $G$ ) is defined similarly, replacing "closed" by "almost closed" (witnessed by $G$ ).

3.5 Claim. Let $\mathbf{C}$ be a local (or revised local) $\lambda$-construction framework.

1) If $\left(\bar{M}^{\ell}, \mathbf{f}^{\ell}\right) \in K_{\lambda}^{\mathrm{qr}}$ for $\ell=1,2$ and $E$ is a club of $\lambda^{+}$and $(*)$ below then $\left(\bar{M}^{1}, \mathbf{f}^{1}\right) \leq\left(\bar{M}^{2}, \mathbf{f}^{2}\right)$ when

$(*)$ if $\delta \in E$ then $M_{\delta+i}^{1}=M_{\delta+i}^{2}$ for $i \leq \mathbf{f}^{1}(\delta)$ and $\mathbf{f}^{1}(\delta) \leq \mathbf{f}^{2}(\delta)$; in the "revised local" version assume in addition that $M^{1}=M^{2}$.

2) $\leq$ is transitive and a reflexive relation on $K_{\lambda}^{q r}$.

3) Any increasing continuous sequence of pairs from $K_{\lambda}^{q r}$ of length $<\lambda^{+}$has a minimal upper bound.

4) If in addition $\mathbf{C}$ is explicitly local (see Definition 3.4(11)) then any increasing sequence in $K_{\lambda}^{q r}$ of length $<\lambda^{+}$has a lub.

5) $\mathbf{C}$ is $\lambda$-nice (hence in particular, $\lambda$-closed).

Proof. 1) Check clause (b) of Definition 3.4(7) and Definition 3.4(a).

2) Use clauses (b,c) of Definition 3.4(7)). In (c) take $\zeta^{*}=2$

3 ) Without loss of generality the elements of the sequence are $\left(\bar{M}^{\xi}, \mathbf{f}^{\xi}\right) \in K_{\lambda}^{\mathrm{qr}}$ for $\xi<\mu$, where $\mu$ is a regular cardinal $\leq \lambda$. For $\xi<\zeta<\mu$, let $E_{\xi, \zeta}$ be a closed unbounded subset of $\lambda$ exemplifying Definition 3.4(4) for $\left(\bar{M}^{\xi}, \mathbf{f}^{\xi}\right) \leqq\left(\bar{M}^{\zeta}, \mathbf{f}^{\zeta}\right)$. First assume $\mu<\lambda$. Let $E \subseteq \bigcap_{\xi<\zeta<\mu} E_{\xi, \zeta} \subset \lambda$ be a closed unbounded subset of $\lambda$, such that: $\left.\alpha \in E \Rightarrow \alpha+\sup _{\xi<\mu} f^{\xi}(\alpha)\right)+1<\operatorname{Min}(E \backslash(\alpha+1))$. Let $E=\left\{\alpha_{i}: i<\lambda\right\}$ with $\alpha_{i}$ increasing continuously with $i$. Notice that for every $i, \xi<\zeta<\mu \Rightarrow \mathbf{f}^{\xi}\left(\alpha_{i}\right) \leqq$ $\mathbf{f}^{\zeta}\left(\alpha_{i}\right)$. Let $E^{*}=\left\{i: \alpha_{i}=i\right\}$.

We now define $\bar{M}=\left\langle M_{j}: j<\lambda\right\rangle$ by defining $M_{j}$ by induction on $j$. If $j=\alpha_{j} \in E^{*}$ let $M_{j}=\bigcup_{\xi<\mu} M_{j}^{\xi}$. If $\alpha<j \leq \alpha+\mathbf{f}^{\xi}(\alpha)$ for some $\alpha \in E^{*}$ and some $\xi<\mu$, let 
$\xi^{\prime}=\sup \left(\xi: \alpha+\mathbf{f}^{\xi}(\alpha) \leq j\right)$ and set $M_{j}=\bigcup_{\xi>\xi^{\prime}} M_{j}^{\xi}$. If $j=\sup _{\xi}\left(\alpha+\mathbf{f}^{\xi}(\alpha)\right)$ for some $\alpha \in E$ and $j>\alpha+\mathbf{f}^{\xi}(\alpha)$ for each $\xi<\mu$. Let $M_{j}=\bigcup_{\beta<j} M_{\beta}$. Finally, if $j<\lambda$ does not fall under any of the previous cases, let $M_{j}=\bigcup_{\xi<\mu} M_{\alpha_{j}}^{\xi}$.

We claim that $\bar{M} \in \mathbf{S} e q_{\lambda}$. One checks that $\bar{M}$ is continuous and increasing, the main point being that if $\alpha \in E^{*}$ and $\alpha<j_{1}<\alpha+f^{\xi_{1}}(\alpha) \leq j_{2}<\alpha+f^{\xi_{2}}(\alpha)$ with $\xi_{1}<\xi_{2}<\mu$, then $M_{j_{1}}^{\xi_{1}} \leq M_{j_{1}}^{\xi_{2}} \leq M_{j_{2}}^{\xi_{2}}$. One must also check that $\left\langle M_{j}, M_{j+1}\right\rangle \in \mathbf{S} e q_{2}$ for all $j$. This follows from clause (c) of Definition 3.4(7).

Let $\mathbf{f}$ be defined by $\mathbf{f}\left(\alpha_{i}\right)=\sup \left\{\mathbf{f}^{\xi}\left(\alpha_{i}\right): \xi<\mu\right\}$ if $i \in E^{*}$ and $\mathbf{f}\left(\alpha_{i}\right)=0$ otherwise. Clearly $\left(\bar{M}^{\xi}, \mathbf{f}^{\xi}\right) \leq(\bar{M}, \mathbf{f})$ for $\xi<\mu$.

What about being a $\leq$-m.u.b.? Assume that $\left(\bar{M}^{\prime}, \mathbf{f}^{\prime}\right) \in K_{\lambda}^{q r}$ and $\xi<\mu \Rightarrow$ $\left(\bar{M}^{\xi}, \mathbf{f}^{\xi}\right) \leq\left(\bar{M}^{\prime}, \mathbf{f}^{\prime}\right)$. So for each $\xi<\mu$ some club $E_{\xi}^{\prime}$ of $\lambda$ exemplifies Definition 3.4(4), and let $E^{\prime}=: \bigcap_{\xi<\mu} E_{\xi}^{\prime} \cap E^{*}$, a club of $\lambda$.

Now for $\delta \in E^{\prime}$ we have $(\forall \xi<\mu)\left(\mathbf{f}^{\xi}(\delta) \leq \mathbf{f}^{\prime}(\delta)\right)$ hence $\mathbf{f}(\delta)=\operatorname{supf}_{\xi<\mu}{ }^{\xi}(\delta) \leq \mathbf{f}^{\prime}(\delta)$, so $\delta \in E^{\prime} \Rightarrow \mathbf{f}(\delta) \leq \mathbf{f}^{\prime}(\delta)$. Similarly $\delta \in E^{\prime} \quad \& \quad j \leq \mathbf{f}(i) \Rightarrow M_{\delta+j} \leq_{\mathfrak{K}} M_{\delta+j}^{\prime}$. So clearly $(\bar{M}, \mathbf{f}) \leq\left(\bar{M}^{\prime}, \mathbf{f}^{\prime}\right)$ and $(\bar{M}, \mathbf{f})$ is a minimal u.b. (see Definition $3.4(10)$ ).

4) As in the proof of part (3), let $\left\langle\left(\bar{M}^{\xi}, \mathbf{f}^{\xi}\right): \xi<\mu\right\rangle$ be as therein and let $(\bar{M}, \mathbf{f})$ be constructed as above. For proving it is a lub, let $\left(M^{\xi}, \mathbf{f}^{\xi}\right) \leq\left(\bar{M}^{\prime}, \mathbf{f}^{\prime}\right)$ for $\xi<\mu$, and define $E^{\prime}$ as there. For $\delta \in E^{\prime}, j<\mathbf{f}(\delta)$ we have $\left\langle\left(M_{\delta+j}^{\xi}, M_{\delta+j+1}^{\xi}\right): \xi \in\left(\xi_{\delta, j+1}, \mu\right)\right\rangle$ is $\leq_{\{[0,1]\}}^{*}$-increasing continuous and $\left\langle M_{\delta+j}^{\xi}, M_{\delta+j+1}^{\xi}\right\rangle \leq_{\{[0,1]\}}^{*}\left(M_{\delta+j}^{\prime}, M_{\delta+j+1}^{\prime}\right)$ for $\xi \in\left(\xi_{\delta, j+1}, \mu\right)$, so as $\mathbf{C}$ is explicitly local by clause (d) in Definition 3.4(11) we have $\left\langle M_{\delta+j}, M_{\delta+j+1}\right\rangle=\left\langle\bigcup_{\xi \in\left(\xi_{\delta, j+1}, \mu\right)} M_{\delta+j}^{\xi}, \bigcup_{\xi \in\left(\xi_{\delta, j+1}, \mu\right)} M_{\delta+j+1}^{\xi}\right\rangle \leq_{\{[0,1]\}}\left(M_{\delta+j}^{\prime}, M_{\delta+j+1}^{\prime}\right)$ is as required.

The proof for the case $\mu=\lambda$ is similar, using diagonal intersection.

5) Left to the reader.

It may clarify matters if we introduce some natural cases of $\mathbf{C}$. We shall use the forthcoming $\mathbf{C}^{0}$ in our construction of many models in $\mathfrak{K}_{\lambda^{+2}}$.

3.6 Definition. For $\ell \in\{0,1,2\}$ and $\lambda=\operatorname{cf}(\lambda)>L S(\mathfrak{K})$, let $\mathbf{C}=\mathbf{C}_{\mathfrak{K}, \lambda}^{\ell}$ consist of

(a) $\tau^{+}=\tau$, $\mathfrak{K}^{+}=\left\{M \in \mathfrak{K}_{<\lambda}:\right.$ if $\lambda$ is a successor cardinal then $\left.\|M\|^{+}=\lambda\right\}$ (with $={ }^{M}$ being equality)

(b) $\mathbf{S} e q_{\alpha}=\left\{\bar{M}: \bar{M}=\left\langle M_{i}: i<\alpha\right\rangle\right.$ is a $\leq_{\mathfrak{K}}$-increasing continuous sequence of members of $\mathfrak{K}_{<\lambda}$ and if $\alpha=\lambda$ then $\bigcup_{i<\alpha} M_{i}$ has cardinality $\left.\lambda\right\}$

(c) $\bar{M}<_{t}^{*} \bar{N}$ when:

( $\alpha) \quad \bar{M}=\left\langle M_{i}: i<\alpha^{*}\right\rangle, \bar{N}=\left\langle N_{i}: i<\beta^{*}\right\rangle$ are from $\mathbf{S e q}$ 
( $\beta)$ if $\left[\gamma_{1}, \gamma_{2}\right] \in t$ then:

(i) $\quad \gamma \in\left[\gamma_{1}, \gamma_{2}\right] \Rightarrow M_{\gamma} \leq_{\mathfrak{K}} N_{\gamma}$

(ii) if $\ell=1$, then in addition $\gamma \in\left[\gamma_{1}, \gamma_{2}\right) \Rightarrow M_{\gamma}=M_{\gamma+1} \cap N_{\gamma}$

(iii) if $\ell=2$, then in addition

$$
\gamma \in\left[\gamma_{1}, \gamma_{2}\right) \Rightarrow M_{\gamma}=M \cap N_{\gamma} \text { where } M=\bigcup_{i<\alpha^{*}} M_{i}
$$

Note that $\mathbf{C}_{\mathfrak{K}, \lambda}^{1}$ is interesting when we have disjoint amalgamation in the appropriate cases.

\subsection{Fact.}

1) If $\ell=0$ or 1 , then $\mathbf{C}_{\mathfrak{K}, \lambda}^{\ell}$ is an explicitly local $\lambda$-construction framework, (hence $\lambda$-nice by $3.5(5))$ and $\mathfrak{K}^{+}$satisfies axioms I-VI.

2) If $\ell=0$ or 2 , then $\mathbf{C}_{\mathfrak{K}, \lambda}^{\ell}$ is an explicitly revised local $\lambda$-construction framework (hence $\lambda$-nice by $3.5(5)$ ) and $\mathfrak{K}^{+}$satisfies axioms I-VI.

3.8 Definition. If $\lambda=\operatorname{cf}(\lambda)>L S(\mathfrak{K})$ then $\mathbf{C}_{\mathfrak{K}, \lambda}^{3}$ consists of

(a) $\tau^{+}=\tau \cup\{P,<\}, \mathfrak{K}^{+}$is the set of $\left(M, P^{M},<^{M}\right)$ where $M \in \mathfrak{K}_{<\lambda}, P^{M} \subseteq$ $M,<^{M}$ a linear ordering of $P^{M}$ (but $={ }^{M}$ may be as in 3.1(2)) and $M_{1} \leq_{\mathfrak{K}^{+}}$ $M_{2}$ iff $\left(M_{1}\lceil\tau) \leq_{\mathfrak{K}}\left(M_{2}\lceil\tau)\right.\right.$ and $M_{1} \subseteq M_{2}$

(b) $\mathbf{S} e q_{\alpha}=\left\{\bar{M}: \bar{M}=\left\langle M_{i}: i \leq \alpha\right\rangle\right.$ is an increasing continuous sequence of members of $\mathfrak{K}^{+}$and $\left\langle M_{i}\lceil\tau: i \leq \alpha\rangle\right.$ is $\leq_{\mathfrak{K}^{-} \text {-increasing, and for }}$

$i<j<\alpha: P^{M_{i}}$ is a proper initial segment of $\left(P^{M_{j}},<{ }^{M_{j}}\right)$ and there is a first element in the difference

we denote the $<^{M_{i+1}}$-first element of $P^{M_{i+1}} \backslash P^{M_{i}}$, by $a_{i}[\bar{M}]$

(d) $\bar{M}<_{t}^{*} \bar{N} \underline{\text { iff }}$

$\bar{M}=\left\langle M_{i}: i\left\langle\alpha^{*}\right\rangle, \bar{N}=\left\langle N_{i}: i<\alpha^{* *}\right\rangle\right.$ are from $\mathbf{S} e q, t$ is a set of pairwise disjoint closed intervals of $\alpha^{*}$ and for any $[\alpha, \beta] \in t$ we have $\left(\beta<\alpha^{*}\right.$ and):

$\gamma \in[\alpha, \beta] \Rightarrow M_{\gamma} \leq_{\mathfrak{K}} N_{\gamma} \& a_{\gamma}[\bar{M}] \notin N_{\gamma}$, moreover

$a_{\gamma}[\bar{M}]=a_{\gamma}[\bar{N}]$.

3.9 Fact. $\mathbf{C}_{\mathfrak{K}, \lambda}^{3}$ is an explicitly local and revised local $\lambda$-construction framework (hence $\lambda$-nice by $3.5(5)$ ) and $\mathfrak{K}^{+}$satisfies axioms I-VI.

We now introduce amalgamation choice functions. The use of " $F$ a $\lambda$-amalgamation choice function" is to help use the weak diamond, by taking out most of the freedom in choosing amalgams. This gives possibilities for coding $(3.14,3.17)$.

3.10 Definition. 1) We say that $F$ is a $\lambda$-amalgamation choice function for the construction framework $\mathbf{C}$ if $F$ is a five place function satisfying:

(a) if $M_{\ell} \in K_{<\lambda}^{+}$for $\ell<3, M_{0} \leq_{\mathfrak{K}^{+}} M_{1}, M_{0} \leq_{\mathfrak{K}^{+}} M_{2}, M_{1} \cap M_{2}=M_{0}$ (before dividing by $\left.={ }^{M_{\ell}}\right) ; a \in M_{2}$ and $\left(\forall b \in M_{0}\right)\left[\neg a={ }^{M_{1}} b\right]$; and $A$ is a set such that $A \cup\left|M_{1}\right| \cup\left|M_{2}\right|$ is a set of ordinals then

$F\left(M_{0}, M_{1}, M_{2}, A, a\right)$, if defined, is a member $N$ of $\mathfrak{K}^{+}$with universe

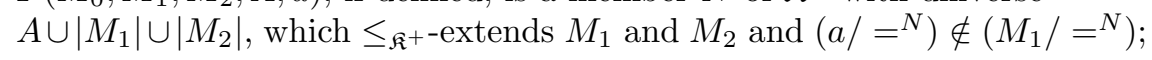


(b) [uniqueness]

if $\left(M_{0}, M_{1}, M_{2}, A, a\right)$ and $\left(M_{0}^{\prime}, M_{1}^{\prime}, M_{2}^{\prime}, A^{\prime}, a^{\prime}\right)$ are as above and in the domain of $F, f$ is an order preserving mapping from $A \cup\left|M_{1}\right| \cup\left|M_{2}\right|$ onto $A^{\prime} \cup\left|M_{1}^{\prime}\right| \cup\left|M_{2}^{\prime}\right|$ such that $f \nmid M_{\ell}$ is an isomorphism from $M_{\ell}$ onto $M_{\ell}^{\prime}$ for $\ell=0,1,2$ (so preserving $=^{M_{\ell}}$, and its negation) and $f(a)=a^{\prime}$ then $f$ is an isomorphism from $F\left(M_{0}, M_{1}, M_{2}, A, a\right)$ onto $F\left(M_{0}^{\prime}, M_{1}^{\prime}, M_{2}^{\prime}, A^{\prime}, a^{\prime}\right)$;

(c) if $F\left(x_{0}, x_{1}, x_{2}, x_{3}, x_{4}\right)$ is well defined then $x_{0}, x_{1}, x_{2}, x_{3}, x_{4}$ are as in part (a).

Observe that as $M_{1} \cap M_{2}=M_{0}$ in (a), if we do not have disjoint amalgamation then we are forced to allow $=_{N}$ to be a nontrivial congruence.

2) If $F$ is defined whenever the conditions in part (a) hold and $A \backslash M_{1} \backslash M_{2}$ has large enough cardinality then we say $F$ is full (if $\lambda=\mu^{+}$, it suffices to demand $A \backslash M_{1} \backslash M_{2}$ has cardinality $\mu$ ).

3) We say $F$ has strong uniqueness if

(d) if $\left(M_{0}, M_{1}, M_{2}, A, a\right)$ and $\left(M_{0}^{\prime}, M_{1}^{\prime}, M_{2}^{\prime}, A^{\prime}, a^{\prime}\right)$ are as above and in the domain of $F$ and for $\ell=0,1,2$ we have $R_{\ell}$ is a isomorphism relation $R_{\ell}$ from $M_{\ell}$ onto $M_{\ell}^{\prime}$ such that $R_{0}=R_{1} \cap\left(M_{0} \times M_{0}^{\prime}\right)=R_{2} \cap\left(M_{0} \times M_{0}^{\prime}\right)$ and $|A|=\left|A^{\prime}\right|$, then there is an isomorphism relation $R$ from $M=F\left(M_{0}, M_{1}, M_{2}, A, a\right)$ onto $M^{\prime}=F\left(M_{0}^{\prime}, M_{1}^{\prime}, M_{2}^{\prime}, A^{\prime}, a\right)$ such that $R_{\ell}=R \cap\left(M_{\ell} \times M_{\ell}^{\prime}\right)$.

3.11 Definition. Assume $\mathbf{C}$ is a $\lambda$-construction framework and $F$ is a $\lambda$-amalgamation choice function for $\mathbf{C}$. Let $\left(\bar{M}^{\ell}, \mathbf{f}^{\ell}\right) \in K_{\lambda}^{\mathrm{qr}}$ for $\ell=1,2$.

1) $\left(\bar{M}^{1}, \mathbf{f}^{1}\right)<_{F, a}^{\text {at }}\left(\bar{M}^{2}, \mathbf{f}^{2}\right)$ (if we omit $a$, this means for some $a$; "at" stands for atomic extension; we may write $\leq_{F, a}^{\text {at }}$ instead of $<_{F, a}^{\text {at }}$ ) means that:

(a) $\left(\bar{M}^{1}, \mathbf{f}^{1}\right) \leq\left(\bar{M}^{2}, \mathbf{f}^{2}\right)$

(b) for some club $E$ of $\lambda$, for every $\delta \in E$ taking $e_{\delta}=:\left[\delta, \delta+\mathbf{f}^{1}(\delta)\right]$ we have

(*) if $\beta<\gamma$ are successive members of $e_{\delta}$ then:

$$
M_{\gamma}^{2}=F\left(M_{\beta}^{1}, M_{\gamma}^{1}, M_{\beta}^{2},\left|M_{\gamma}^{2}\right|, a\right)
$$

(**) $\quad\left|M_{\gamma}^{2}\right|=\left|M_{\gamma}^{1}\right| \cup\left|M_{\beta}^{2}\right| \cup\left\{i: i\right.$ an ordinal not in $\left|M_{\gamma}^{1}\right| \cup\left|M_{\beta}^{2}\right|$ and $\operatorname{otp}\left(\left|M_{\gamma}^{2}\right| \cap\right.$ $\left.i \backslash\left|M_{\gamma}^{1}\right| \cup\left|M_{\beta}^{2}\right|\right)<\left\|M_{\gamma}^{2}\right\|$

$(* * *) \quad\left\|M_{\gamma}^{2}\right\|=\operatorname{Min}\left\{\|N\|: N=F\left(M_{\beta}^{1}, M_{\delta}^{1}, M_{\beta}^{2},|N|, a\right)\right\}$.

A suitable club $E$ may be called a witness for the relation. Implicit in clause (b) in $a \in M^{2}$ and $\neg(\exists b)\left(b \in M^{1} \& a=M^{2} b\right)$.

2) $\left(\bar{M}^{\prime}, \mathbf{f}^{\prime}\right) \leq{ }_{F}\left(\bar{M}^{\prime \prime}, \mathbf{f}^{\prime \prime}\right)$ means that: there is a sequence $\left\langle\left(\bar{M}^{\zeta}, \mathbf{f}^{\zeta}\right): \zeta \leq \xi\right\rangle$ such that:

(a) $\xi<\lambda$

(b) $\left(\bar{M}^{\zeta}, \mathbf{f}^{\zeta}\right) \in K_{\lambda}^{\mathrm{qr}}$ is $\leq$-increasing continuous in $\zeta$ (remember Definition 3.4(10))

(c) for each $\zeta<\xi$ we have $\left(\bar{M}^{\zeta}, \mathbf{f}^{\zeta}\right) \leq_{F}^{\text {at }}\left(\bar{M}^{\zeta+1}, \mathbf{f}^{\zeta+1}\right)$

(d) $\left(\bar{M}^{\prime}, \mathbf{f}^{\prime}\right)=\left(\bar{M}, \mathbf{f}^{0}\right)$ and $\left(\bar{M}^{\prime \prime}, \mathbf{f}^{\prime \prime}\right)=\left(\bar{M}^{\xi}, \mathbf{f}^{\xi}\right)$. 
A club $E$ which witnesses all the relations in (c) is called a witness for the relation $\leq_{F}$.

3) $<_{F, a}^{\text {at,* }}$ is defined similarly to part (1) but we demand in clause (b) only that $e_{\delta} \subseteq\left[\delta, \delta+\mathbf{f}^{2}(\delta)\right]$ is closed and $\left\{\delta, \delta+\mathbf{f}^{2}(\delta)\right\} \subseteq e_{\delta}$; the requirement from clause (a) is unchanged and we require also:

$$
\text { if } \beta \in\left[\delta, \delta+\mathbf{f}^{2}(\delta)\right] \text { then } M_{\beta}^{2}=M_{\max \left(e_{\delta} \cap(\beta+1)\right)}^{2} \text {. }
$$

Then define $\leq_{F}^{*}$ by iterating $\leq_{F}^{a t, *}$.

4) We may replace $F$ by $\mathbf{F}$, a family of such functions. Then in each case in $3.11(2)(\mathrm{c})$ we use one such that $F$ 's. $\mathbf{F}^{*}$ is the family of all such $F$ 's.

5) $\left(\bar{M}^{1}, \mathbf{f}^{1}\right)<_{F, a}\left(\bar{M}^{2}, \mathbf{f}^{2}\right)$ means that for some $(\bar{M}, \mathbf{f})$ we have $\left(\bar{M}^{1}, \mathbf{f}^{1}\right) \leq_{F, a}^{a t}$ $(\bar{M}, \bar{f}) \leq_{F}\left(\bar{M}^{2}, \mathbf{f}^{2}\right)$.

6) We define mub as in 3.4(10).

3.12 Remark. 1) What we prove below on $<_{F, a}^{\mathrm{at}}, \leq_{F}$ also holds for $<_{F, a}^{\mathrm{at}, *}, \leq_{F}^{*}$.

2) Note: using $\mathbf{F}$ rather than $F$ may help in proving cases of Definition 3.20, but we can use one $F$ which codes all members of $\mathbf{F}$ by asking on $A \backslash M_{1} \backslash M_{2}$, though artificially.

3) We can replace $F$ by $\left\langle F_{\eta}: \eta \in \lambda\right.$ a sequence of ordinals of length $<\lambda, \eta(1+i)<$ $\left.2, \eta(0)<2^{<\lambda}\right\rangle$, each $F_{\eta}$ with uniqueness 3.10(3) and (**) of 3.11(1)(b) is replaced by $M_{\gamma}^{2}=f_{\eta\lceil\delta}\left(M_{\beta}^{1}, M_{\gamma}^{1}, M_{\beta}^{2},\left|M_{\gamma}^{2}\right|, a\right)$, and omit $(* * *)$ there.

3.13 Claim. If $\mathbf{C}$ is nice, then on $K_{\lambda}^{\mathrm{qr}}, \leq_{F}$ is a quasi-order, and every increasing continuous sequence of length less than $\lambda^{+}$has a mub.

Proof. Check.

3.14 Definition. 1) We say a $\lambda$-amalgamation choice function $F$ for $\mathfrak{K}^{+}$has the $\lambda$ coding property for $\mathbf{C}$ if: $\mathbf{S} e q_{\lambda} \neq \emptyset$, and for every $\bar{M}^{1} \in \mathbf{S} e q_{\lambda}$, function $\mathbf{f}^{1}: \lambda \rightarrow \lambda$, and $S \subseteq \lambda$ we can find $\bar{M}^{2, \eta} \in \mathbf{S} e q_{\lambda}$ for $\eta \in{ }^{\lambda} 2$ with $\eta$ extending $0_{\lambda \backslash S}$ that is $\eta \uparrow(\lambda \backslash S)$ being constantly zero, a function $\mathbf{f}^{2}: \lambda \rightarrow \lambda$ such that $\mathbf{f}^{1} \leq \mathscr{D}_{\lambda} \mathbf{f}^{2}, \mathbf{f}^{2} \uparrow$ $(\lambda \backslash S)=\mathbf{f}^{1} \uparrow(\lambda \backslash S)$ and an element $a_{\eta}$ of $M^{2, \eta}$ (usually $a_{\eta}=a$ ) such that:

$(*)_{1}\left(\bar{M}^{1}, \mathbf{f}^{1}\right) \leq_{F, a}^{\text {at }}\left(\bar{M}^{2, \eta}, \mathbf{f}^{1}\right)$ for all $\eta$ extending $0_{\lambda \backslash S}$, and $\eta\left\lceil\alpha=\nu \uparrow \alpha \Rightarrow M^{2, \eta}\left\lceil\alpha=M^{2, \nu}\lceil\alpha\right.\right.$

$(*)_{2}$ for some club $E$ of $\lambda$ the following is impossible: for some $\eta_{3}, \eta_{4} \in{ }^{\lambda_{2}}$ extending $0_{\lambda \backslash S}$, for $\ell=3,4$ we have $\left(\bar{M}^{1}, \mathbf{f}^{2}\right) \leq_{F, a^{\ell}}\left(\bar{M}^{\ell}, \mathbf{f}^{\ell}\right)$ witnessed by a club $E_{\ell}$ we have (abusing our notation we are dividing by the equality congrugence) $f_{\ell}$ a $\leq_{\mathfrak{K}}$-embedding of $M^{2, \eta_{\ell}} \uparrow \tau$ into $M^{\ell} \uparrow \tau$ over $M_{\lambda}^{1}$, and for some $\delta \in E \cap E_{3} \cap E_{4} \cap S$ we have $\bar{M}^{3}\left\lceil\delta=\bar{M}^{4}\left\lceil\delta, a^{3} \in M_{\delta}^{2, \eta_{3}}, a^{4} \in\right.\right.$ $M_{\delta}^{2, \eta_{4}}, f_{3}\left(a^{3}\right)=f_{4}\left(a^{4}\right), \mathbf{f}^{3} \uparrow \delta=\mathbf{f}^{4} \uparrow \delta, f_{3} \uparrow M_{\delta}^{2, \eta_{3}}=f_{4} \uparrow M_{\delta}^{2, \eta_{4}}$, and $\ell \in\{3,4\} \Rightarrow \operatorname{Rang}\left(f_{\ell}\left\lceil M^{2, \eta_{\ell}}\right) \subseteq M_{\delta}^{\ell}, \eta_{3}\left\lceil\delta=\eta_{4}\left\lceil\delta, \eta_{3}(\delta) \neq \eta_{4}(\delta)\right.\right.\right.$.

2) We say that $F$ has the weak $\lambda$-coding property if above we restrict ourselves to the cases $\mathbf{f}^{1}\left\lceil S=0_{S}\right.$. We can even restrict ourselves to the cases $\mathbf{f}^{1} \in \mathscr{F} \subseteq{ }^{\lambda} \lambda$ provided that $0_{\lambda} \in \mathscr{F}$ and we demand $\mathbf{f}^{2} \in \mathscr{F}$. 
3) If we replace $F$ by $\mathbf{F}$, a family of such functions it means we use Definition $3.11(4)$.

4) We say $\mathfrak{K}$ has a coding property if some $\lambda$-amalgamation choice function $F$ has this property. Typically the actual choice of $F$ is irrelevant as long as its domain is sufficiently rich.

3.15 Observation. The restriction above to $\eta$ such that $\eta$ extends $0_{\lambda \backslash S}, S$ is natural but inessential, as we can extend the definition of $M^{2, \eta}$ to all $\eta$ in ${ }^{\lambda} 2$ by defining $M^{2, \eta}=M^{2, \eta^{\prime}}$ where $\eta^{\prime} \uparrow S=\eta \uparrow S$ and $\eta^{\prime} \uparrow(\lambda \backslash S)$ is constantly zero. Then the same properties will hold.

\subsection{Remark.}

1) For a local construction framework $\mathbf{C}$ in $3.14(1)$ the conditions $(*)_{1}$ and $(*)_{2}$ can be replaced by local requirements. For example, in condition $(*)_{1}$, we may take $3.4(7 \mathrm{~b})$ into account.

2) In $3.14(*)_{2}$ a sufficient condition for the impossibility of the stated conditions on $E, \eta^{3}, \eta^{4}$ and $\delta$, where $\eta^{3} \uparrow \delta=\eta^{4} \uparrow \delta=\eta$, say, is that there is $\bar{a} \in M_{\delta}^{2, \eta}$ so that:

$$
(*)_{3} \operatorname{tp}\left(\bar{a}, M_{\delta+\mathbf{f}^{2}(\delta)}^{1}, M^{2, \eta^{3}}\right) \neq \operatorname{tp}\left(\bar{a}, M_{\delta+\mathbf{f}^{2}(\delta)}^{1}, M^{2, \eta^{4}}\right) .
$$

We can even allow $\bar{a}$ to be infinite here, say a full listing of $M_{\delta}^{2, \eta}$.

To see that this suffices, suppose that we also have the conditions of $3.14(*)_{2}$. Then for $\ell=3,4$ as $M_{\delta+j+1}^{2, \eta_{\ell}}$ is given by

$$
F\left(M_{\delta+j}^{1}, M_{\delta+j+1}^{1}, M_{\delta+j}^{2, \eta_{\ell}},\left|M_{\delta+j+1}^{2, \eta_{\ell}}\right|, a^{\ell}\right)
$$

where $j<\mathbf{f}^{2}(\delta)$, we find $M_{\delta+\mathbf{f}^{2}(\delta)}^{2, \eta_{3}}=M_{\delta+\mathbf{f}^{2}(\delta)}^{2, \eta_{4}}=M^{*}$, say.

Since $M^{2, \eta_{3}}$ and $M^{2, \eta_{4}}$ can be amalgamated over $M^{*}$, we have

$$
\operatorname{tp}\left(f_{3}(\bar{a}), M_{\delta+\mathbf{f}^{2}(\delta)}^{1}, M^{3, \eta_{3}}\right)=\operatorname{tp}\left(f_{4}(\bar{a}), M_{\delta+\mathbf{f}^{2}(\delta)}^{1}, M^{4, \eta_{4}}\right) .
$$

On the other hand by $(*)_{2}$ we have

$$
\operatorname{tp}\left(\bar{a}, M_{\delta+\mathbf{f}^{2}(\delta)}^{1}, M^{2, \eta_{\ell}}\right)=\operatorname{tp}\left(f_{\ell}(\bar{a}), M_{\delta+\mathbf{f}^{2}(\delta)}^{1}, M^{\ell, \eta_{\ell}}\right)
$$

and this gives a contradiction.

3) To understand Definitions 3.14(1,2) you may look at the places they are verified, such as 3.25 and 3.27 . Also see $3.21(3,4)$.

4) The next lemma deduces from the criterion in 3.14(1) another one which is natural for use in a non-structure theorem.

5) Note that 3.14(1) implies: for every $\left(\bar{M}^{1}, \mathbf{f}^{1}\right)$ there is $\left(\bar{M}^{2}, \mathbf{f}^{2}\right)$ such that $\left(\bar{M}^{1}, \mathbf{f}^{1}\right) \leq_{F, a}^{a t}\left(\bar{M}^{2}, \mathbf{f}^{2}\right)$ and $M_{\lambda}^{1} \neq M_{\lambda}^{2}$.

6) In Definition 3.14(1) we can replace $<_{F, a}^{a t}$ by $<_{F}$ or $<_{F, a}$ with no harm as $<_{F, a}$ satisfies the requirement on $<_{F, a}^{a t}$ and starting from it we again get $<_{F}$.

7) In $(*)_{2}$ of Definition 3.14(1) for some function $H$ depending on $\left(\bar{M}^{1}, f^{1}\right)$ we may add the further restriction $\ell \in\{3,4\}$ and $\alpha<\delta$ implies $\delta \in H\left(\bar{M}^{\ell}\left\lceil\alpha, f_{\ell} \uparrow M_{\alpha}^{\ell}\right)\right.$. I.e. this weakening of the demand does not change the desired conclusions. 
8) We can weaken the demand in $(*)_{2}$ of $3.14(1)$ to extensions which actually arise but this seems more cumbersome. While the adaptation is straightforward, we have no application in mind.

9) In $3.14(1),(*)_{2}$ we may strengthen the requirement by excluding the case where the club $E$ is allowed to depend on $\eta$. That is, we consider quadruples $\left(E^{\eta}, \bar{M}^{\eta}, \mathbf{f}^{\eta}, f^{\eta}\right)$ for $\eta \in{ }^{\lambda} 2$ such that $\left(\bar{M}^{1}, \mathbf{f}^{2}\right) \leq_{F}\left(\bar{M}^{\eta}, \mathbf{f}^{\eta}\right)$ is witnessed by a club

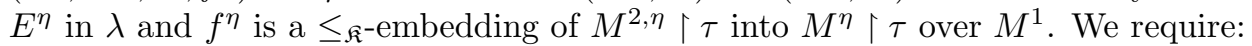

$(*)_{2}^{\prime}$ for no $\eta^{3}, \eta^{4} \in{ }^{\lambda} 2$ and $\delta \in E^{\eta^{3}} \cap E^{\eta^{4}} \cap E \cap S$ do we have:

$$
\begin{gathered}
\eta_{3} \uparrow \delta=\eta_{4}\left\lceil\delta, \eta_{3}(\delta) \neq \eta_{4}(\delta), \mathbf{f}^{3}\left\lceil\delta=\mathbf{f}^{4} \uparrow \delta ;\right.\right. \\
\bar{M}^{2, \eta_{3}} \uparrow\left[\delta, \delta+\mathbf{f}^{2}(\delta)\right]=\bar{M}^{2, \eta_{4}}\left\lceil\left[\delta, \delta+\mathbf{f}^{3}(\delta)\right] ;\right. \\
f^{\eta_{3}}\left\lceil M_{\delta}^{2, \eta_{3}}=f^{\eta_{4}}\left\lceil M_{\delta}^{2, \eta_{4}} ;\right.\right. \\
f^{\eta_{\ell}}\left[M_{\delta}^{2, \eta_{\ell}}\right] \subseteq M_{\delta}^{\eta_{\ell}} \text { for } \ell=3,4 .
\end{gathered}
$$

10) In 3.14 we can also require the models $M^{1}, M^{2, \eta}$ to have universes $\lambda(1+\alpha)$ and $\lambda(1+\alpha+1)$ respectively for some $\alpha$, with $\lambda(1+\alpha) \in M_{0}^{2, \eta}$. This will not change much.

3.17 Lemma. Assume $(\exists \mu)\left(\lambda=\mu^{+} \quad \& \quad 2^{\mu}<2^{\mu^{+}}\right)$or at least the definitional weak diamond on $\lambda$ holds. Assume $\mathbf{C}$ is $\lambda$-nice, $J=\operatorname{WDmId}^{\mathrm{Def}}(\lambda)$.

If the $\lambda$-amalgamation choice function $F$ has the $\lambda$-coding property, then it has the explicit $(\lambda, J)$-coding property, which means: if $\left(\bar{M}^{1}, \overline{\mathbf{f}}^{1}\right) \in K_{\lambda}^{\mathrm{qs}}$ and $\overline{S \subseteq \lambda}$ satisfies $S \notin J \underline{\text { then }}$ we can find $\left(\bar{M}^{2}, \mathbf{f}^{2}\right) \in K_{\lambda}^{\mathrm{qs}}$ such that:

(a) $\left(\bar{M}^{1}, \mathbf{f}^{1}\right) \leq_{F}^{\text {at }}\left(\bar{M}^{2}, \mathbf{f}^{1}\right)$ and $\mathbf{f}^{1} \uparrow(\lambda \backslash S)=\mathbf{f}^{2} \uparrow(\lambda \backslash S)$

(b) if $\left(\bar{M}^{1}, \mathbf{f}^{2}\right) \leq_{F}\left(\bar{M}^{3}, \mathbf{f}^{3}\right) \in K_{\lambda}^{\mathrm{qs}} \underline{\text { then }} M^{2}\left\lceil\tau\right.$ cannot be $\leq_{\mathfrak{K}}$-embedded into $M^{3}\left\lceil\tau\right.$ over $M^{1}$.

Proof. The proof is straight forward once you digest the meaning of weak diamond.

Let $S=\lambda$. Suppose $\left\langle\bar{M}^{2, \eta}: \eta \in{ }^{\lambda} 2\right\rangle$ and $\mathbf{f}^{2}$ are as in 3.14(1), taking note of 3.15. Then we claim there is $\nu \in{ }^{\lambda} 2$ for which 3.17 holds on taking $\bar{M}^{2}$ to be $\bar{M}^{2, \nu}$. Assume toward a contradiction that this fails for each $\nu$. Then clause (b) fails, and for each $\nu \in{ }^{\lambda} 2$ we have some $\bar{M}^{3, \nu}$ and $\mathbf{f}^{3, \nu}$ with:

$$
\begin{gathered}
\left(\bar{M}^{1}, \mathbf{f}^{2}\right) \leq_{F}\left(\bar{M}^{3, \nu}, \mathbf{f}^{3, \nu}\right) \text { witnessed by a club } E^{\nu} ; \\
f_{\nu}: M^{2, \nu}\left\lceil\tau \rightarrow M^{3, \nu} \uparrow \tau \text { over } M^{1} \uparrow \tau \text { a } \leq_{\mathfrak{K}}\right. \text {-embedding. }
\end{gathered}
$$

Now by the definition of $\operatorname{WDmId}^{\operatorname{Def}}(\lambda)$ we can find $\eta_{3} \neq \eta_{4}$ in ${ }^{\lambda} 2$ and $\delta \in E^{\eta_{3}} \cap$ $E^{\eta_{4}} \cap E^{*} \cap S$ with $E^{*}=\left\{\alpha<\lambda\right.$ limit: $\beta<\alpha$ implies $\left.\beta+\mathbf{f}^{2}(\delta), \beta+\mathbf{f}^{1}(\delta)<\alpha\right\}$, as forbidden in $(*)_{2}$ of $3.14(1)$.

Now we can give a reasonable non-structure theorem. 
3.18 Theorem. Assume $\mathbf{C}$ is $\lambda$-nice, $(\exists \mu)\left(\lambda=\mu^{+} \& 2^{\mu}<2^{\mu^{+}}\right) \& 2^{\lambda}<2^{\lambda^{+}}$, or at least $D W D(\lambda)$, and $D W D^{+}\left(\lambda^{+}\right)$. Let $J=W D m I d^{\operatorname{Def}}(\lambda)$.

If $F$ has the $(\lambda, J)$-coding property, then $I\left(\lambda^{+}, \mathfrak{K}\right) \geq 2^{\lambda^{+}}$; also if $2^{\lambda^{+}}>\left(2^{\lambda}\right)^{+}$ then $\operatorname{IE}\left(\lambda^{+}, \mathfrak{K}\right) \geq 2^{\lambda^{+}}$.

Proof. We choose by induction on $\alpha<\lambda^{+}$, for every $\eta \in{ }^{\alpha} 2$, a pair $\left(\bar{M}^{\eta}, \mathbf{f}^{\eta}\right)$ such that:

(a) $\left(\bar{M}^{\eta}, \mathbf{f}^{\eta}\right) \in K_{\lambda}^{\mathrm{qs}}$

(b) if $\nu \triangleleft \eta$ then $\left(\bar{M}^{\nu}, \mathbf{f}^{\nu}\right) \leq_{F}\left(\bar{M}^{\eta}, \overline{\mathbf{f}}^{\eta}\right)$

(c) $\left(\bar{M}^{\eta}, \mathbf{f}^{\eta}\right) \leq_{F, a_{\eta^{\wedge}\langle 0\rangle}}^{\text {at }}\left(\bar{M}^{\eta^{\wedge}\langle 0\rangle}, \mathbf{f}^{\eta^{\wedge}\langle 0\rangle}\right)$

(d) $\left(\bar{M}^{\eta}, \overline{\mathbf{f}}^{\eta}\right) \leq_{F, a_{\eta^{\wedge}\langle 1\rangle}}^{\text {at }}\left(\bar{M}^{\eta^{\wedge}\langle 1\rangle}, \mathbf{f}^{\eta^{\wedge}\langle 1\rangle}\right)$

(e) if $\ell g(\eta)$ is a limit ordinal and $\left(\bar{M}^{\eta}, \bar{f}^{\eta^{\wedge}\langle 0\rangle}\right) \leq_{F}\left(\bar{M}^{\prime}, \mathbf{f}^{\prime}\right) \underline{\text { then }}$

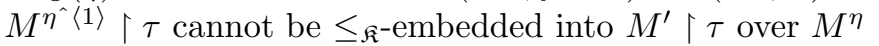

$(f)$ if $\alpha$ is limit ordinal, then $\left(\bar{M}^{\eta}, \mathbf{f}^{\eta}\right)$ is a $\leq_{F}$-mub of $\left\langle\left(\bar{M}^{\eta \uparrow i}, \mathbf{f}^{\eta \uparrow i}\right): i<\alpha\right\rangle$.

For $\alpha=0$ note that as $\mathbf{S} e q_{\lambda} \neq \emptyset$ also $\mathbf{S} e q_{\lambda}^{s} \neq \emptyset$ hence $K_{\lambda}^{\mathrm{qs}} \neq \emptyset$. For $\alpha$ limit use 3.6, 3.10. For $\alpha=\beta+1, \beta$ a limit ordinal and $\nu \in{ }^{\beta} 2$, define $\left(\bar{M}^{\nu^{\wedge}\langle\ell\rangle}, \mathbf{f}^{\nu^{\wedge}\langle\ell\rangle}\right)$ for $\ell=0,1$ by 3.17. If $\alpha=\beta+1, \beta$ non-limit use 3.16(3). Let $M^{\eta}=\bigcup_{\alpha<\lambda^{+}} M^{\eta\lceil\alpha}\lceil\tau$ for $\eta \in{ }^{\left(\lambda^{+}\right)} 2$. Now note $\left\{a_{\eta \uparrow(i+1)} /=M^{M^{\eta}}: i<\lambda^{+}\right\} \subseteq M^{\eta} /=M^{\eta}$ are pairwise distinct so $M^{\eta} \in K_{\lambda^{+}}$. Now we can apply 1.6 (with $\lambda^{+}$here standing for $\lambda$ there). $\quad \square_{3.18}$

Unfortunately, in some interesting cases we get only weak coding.

3.19 Theorem. Assume $\mathbf{C}$ is $\lambda$-nice, $(\exists \mu)\left(\lambda=\mu^{+}\right.$and $2^{\mu}<2^{\lambda}<2^{\lambda^{+}}$and $W D m I d\left(\lambda^{+}\right)$is not $\lambda^{+}$-saturated (or at least $D f W D(\lambda), D f W D^{+}\left(\lambda^{+}\right)$and $W D m I d d^{\operatorname{Def}}(\lambda)$ is not $\lambda^{+}$-saturated)).

If $F$ has the weak $\lambda$-coding property (see Definition 3.14(2)), or at least the parallel of the conclusion of 3.17 , then $I\left(\lambda^{+}, K\right) \geq 2^{\lambda^{+}}$.

Proof. We can find $\left\langle S_{\alpha}^{*}: \alpha<\lambda^{+}\right\rangle$such that:

$$
\begin{gathered}
S_{\alpha}^{*} \subseteq \lambda \\
{\left[\alpha<\beta \Rightarrow\left|S_{\alpha}^{*} \backslash S_{\beta}^{*}\right|<\lambda\right]}
\end{gathered}
$$

and

$$
S_{\alpha}^{\prime}=: S_{\alpha+1}^{*} \backslash S_{\alpha}^{*} \notin \mathrm{WDmId}^{\operatorname{def}}(\lambda) .
$$

We again choose by induction on $\alpha<\lambda^{+}$for every $\eta \in{ }^{\alpha} 2$ a pair $\left(\bar{M}^{\eta}, \mathbf{f}^{\eta}\right)$ such that: 
(a) $\left(\bar{M}^{\eta}, \mathbf{f}^{\eta}\right) \in K_{\lambda}^{\mathrm{qs}}$

(b) if $\nu \triangleleft \eta$, then $\left(\bar{M}^{\nu}, \mathbf{f}^{\nu}\right) \leq_{F}\left(\bar{M}^{\eta}, \mathbf{f}^{\eta}\right)$

(c) $f^{\eta} \uparrow\left(\lambda \backslash S_{\ell g(\eta)}\right)=0_{\lambda \backslash S_{\ell g(\eta)}}$

(d) $\left(\bar{M}^{\eta}, \mathbf{f}^{\eta}\right)<_{F, a_{\eta}\langle 0\rangle}^{\text {at }}\left(\bar{M}^{\eta^{\wedge}\langle 0\rangle}, \mathbf{f}^{\eta^{\wedge}\langle 0\rangle}\right)$

(e) $\left(\bar{M}^{\eta}, \mathbf{f}^{\eta}\right)<_{F, a_{\eta^{\wedge}\langle 1\rangle}}^{\text {at }}\left(\bar{M}^{\eta^{\wedge}\langle 1\rangle}, \mathbf{f}^{\eta}\right)$

$(f)$ if $\left(\bar{M}^{\eta}, \mathbf{f}^{\eta^{\wedge}\langle 0\rangle}\right) \leq_{F}\left(\bar{M}^{\prime}, \mathbf{f}^{\prime}\right)$ then $M^{\eta^{\wedge}\langle 1\rangle} \uparrow \tau$ cannot be $\leq_{\mathfrak{K}^{-} \text {-embedded }}$ into $M^{\prime}\left\lceil\tau\right.$ over $M^{\eta}$

(g) if $\alpha$ is a limit ordinal, then $\left(\bar{M}^{\eta}, \mathbf{f}^{\eta}\right)$ is a $\leq_{F}$-mub of $\left\langle\left(\bar{M}^{\eta \nmid i}, \mathbf{f}^{\eta \nmid i}\right): i<\alpha\right\rangle$.

Again there are no problems (the difference is in clause (c)).

Then we apply 1.6(1) (or 1.7(1)).

3.20 Definition. 1) We say a $\lambda$-amalgamation choice function $F$ for $\mathbf{C}$ has the weak $^{3}$ (or weakly) local $\lambda$-coding property for $\mathbf{C}$ if:

$(*)_{1}$ Assume $\left\langle M_{0}, M_{1}\right\rangle \in \mathbf{S} e q_{2}, M_{0} \leq_{\mathfrak{K}^{+}} N_{0} \in \mathfrak{K}_{<\lambda}^{+}, M_{1} \cup N_{0} \subseteq \lambda^{+}$, and $\left|M_{1}\right| \cap$ $\left|N_{0}\right|=\left|M_{0}\right|$ and $a \in N_{0}, a /={ }^{N_{0}} \notin\left(M_{0} /={ }^{N_{0}}\right)$ (i.e. $\left(\forall b \in M_{0}\right)\left(\neg a={ }^{N_{0}} b\right)$ ). Then we can find $N^{1}, N^{2} \in \mathfrak{K}_{<\lambda}^{+}$such that:

(a) $N^{1}=F\left(M_{0}, M_{1}, N_{0},\left|N^{1}\right|, a\right)$

(b) $\left\langle N_{0}, N^{1}\right\rangle \in \mathbf{S} e q_{2}$ and $\left\langle N_{0}, N^{2}\right\rangle \in \mathbf{S} e q_{2}$

(c) $M_{1} \leq_{\mathfrak{K}^{+}} N^{1}$ and $M_{1} \leq_{\mathfrak{K}^{+}} N^{2}$

(d) $N^{1} \uparrow \tau, N^{2} \uparrow \tau$ are contradicting ${ }^{4}$ amalgamations of $M_{1}\left\lceil\tau, N_{0} \uparrow \tau\right.$ over $M_{0} \uparrow \tau$; i.e. for no $N^{\prime}, h$ do we have: $\left(N^{1} \uparrow \tau\right) \leq_{\mathfrak{K}} N^{\prime} \in \mathfrak{K}_{<\lambda}$ and

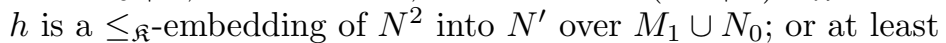

$(\mathrm{d})^{-}\left(N^{1}, N^{2}\right)$ is a $\tau$-contradicting pair of amalgamations of $M_{1}, N_{0}$ over $M_{0}$ which just says: if $N^{1} \leq_{\mathfrak{K}^{+}} N \in \mathfrak{K}_{<\lambda}^{1}$ then there is no $\leq_{\mathfrak{K}^{-} \text {-embedding }}$ $h$ of $N^{2} \uparrow \tau$ into $N \uparrow \tau$ over $M_{1} \cup N_{0}$ (i.e. is the identity on $M_{1}$ and on $N_{0}$ )

(note: this is not necessarily symmetric; and we use just the $\tau$-reducts of $N^{2}, M_{0}$, $M_{1}, N_{0}$ so we can replace them by

$N^{2}\left\lceil\tau, M_{0}\left\lceil\tau, M_{1}\left\lceil\tau, N_{0}\lceil\tau\right.\right.\right.$ respectively).

2) We say that a $\lambda$-amalgamation choice function $F$ for $\mathbf{C}$ has the local $\lambda$-coding property if:

$(*)_{2}$ if $\bar{M}=\left\langle M_{j}: j<\lambda\right\rangle \in \mathbf{S} e q_{\lambda}, \bar{N}=\left\langle N_{j}: j \leq \delta+i\right\rangle \in \mathbf{S} e q_{\delta+i}, a \in N_{0}$,

$\left(a /={ }^{N_{\delta+i}}\right) \notin M_{\delta+i} /={ }^{M_{\delta+i}}$ and

$\bar{M} \uparrow(\delta+i+1) \leq_{\{[\delta, \delta+i]\}}^{*} \bar{N}$, and

$N_{\delta+j+1}=F\left(M_{\delta+j}, M_{\delta+j+1}, N_{\delta+j},\left|N_{\delta+j+1}\right|, a\right)$ for $j<i$ then for some $i_{1}, i_{2} \in(i, \lambda)$ and $\bar{N}^{\ell}=\left\langle N_{\alpha}^{\ell}: \alpha<\delta+i_{\ell}\right\rangle \in \mathbf{S} e q_{\delta+i_{\ell}}$ for $\ell=1,2$ we have:

(a) $\bar{N}^{\ell} \uparrow(\delta+i+1)=\bar{N}$ for $\ell=1,2$

\footnotetext{
${ }^{3}$ there is no clear relation between "weak local" and "local" $\lambda$-coding inspite of the name

${ }^{4}$ Of course, we may consider only ones in "legal" extensions. We can also note that for the intended use, the disjointness is automatic (so 3.1(2) not needed)
} 
(b) for $j \in\left[\delta+i, \delta+i_{1}\right)$ we have

$N_{j+1}^{1}=F\left(M_{j}, M_{j+1}, N_{j}^{1},\left|N_{j+1}^{1}\right|, a\right)$

(c) $M_{\delta+i_{2}} \leq_{\mathfrak{K}^{+}} N_{\delta+i_{2}}^{2}$

(d) $N_{\delta+i_{1}}^{1} \uparrow \tau, N_{\delta+i_{2}}^{2} \uparrow \tau$ are contradictory amalgamations of $M_{\delta+i_{1}}\left\lceil\tau\right.$ and $N_{\delta}\left\lceil\tau\right.$ over $M_{\delta}\lceil\tau$ or at least

(d) ${ }^{-}\left(N_{\delta+i_{1}}^{1}, N_{\delta+i_{2}}^{2}\right)$ are $\tau$-contradictory amalgamations of $M_{\delta+i(*)}$ and $N_{\delta}$ over $M_{\delta}$ where $i(*)=\operatorname{Min}\left\{i_{1}, i_{2}\right\}$.

(So if $i=0$, this gives us a possibility to amalgamate, helpful for $\left.i \in \lambda \backslash \bigcup_{\delta \in E}[\delta, \delta+\mathbf{f}(\delta))\right)$.

3) We say that a $\lambda$-amalgamation choice function $F$ for $\mathbf{C}$ has the weaker local $\lambda$-coding property for $\mathbf{C}$ if:

$(*)_{3}$ as in part (2) but $i=0$.

4) In 1), 2), 3) above we say $\mathbf{C}$ has weak local or the local or the weaker local coding property respectively, if we omit the mention of $F$ meaning for some $F$ (clause (a) in $(*)_{1}$, clause $(\mathrm{b})$ in $(*)_{2}$.)

3.21 Claim. 1) If $F$ has the weak local $\lambda$-coding property for $\mathbf{C}$ or $F$ has the local $\lambda$-coding property for $\mathbf{C}$ then $F$ has the weaker local $\lambda$-coding property for $\mathbf{C}$.

2) Assume

(a) $\mathbf{C}$ has a local $\lambda$-construction framework

(b) $F$ has the $\lambda$-coding (or weaker $\lambda$-coding) (or the weak $\lambda$-coding) property for C.

Then for some $F^{\prime}$ we have:

$(\alpha) F^{\prime}$, too, is a $\lambda$-amalgamation choice function for $\mathbf{C}$

( $\beta)$ if $F\left(N_{0}, N_{1}, N_{2}, A, a\right)$ is well defined and its $\tau$-reduct is $<_{\mathfrak{K}} M \in K_{<\lambda}$ and $A \subseteq A^{\prime} \subseteq \lambda^{+},\left|A^{\prime}\right|<\lambda$, then for some $A^{\prime \prime}, A^{\prime} \subseteq A^{\prime \prime} \subseteq \lambda^{+},\left|A^{\prime \prime}\right|<$ $\lambda$, and $F^{\prime}\left(N_{0}, N_{1}, N_{2}, A^{\prime}, a\right)$ is well defined and $F\left(N_{0}, N_{1}, N_{2}, A, a\right) \leq_{\mathfrak{K}^{+}}$ $F^{\prime}\left(N_{0}, N_{1}, N_{2}, A^{\prime \prime}, a\right)$ and $M \leq_{\mathfrak{K}} F^{\prime}\left(N_{0}, N_{1}, N_{2}, A^{\prime \prime}, a\right)\lceil\tau$

$(\gamma) F^{\prime}$ has the local or weak local or weaker local (respectively as in (b)) $\lambda$-coding property for $\mathbf{C}$.

3) If $\mathbf{C}$ is local ( $\lambda$-construction framework), $F$ a $\lambda$-amalgamation choice function, with the weak (or just weaker) local $\lambda$-coding property, then $F$ has the weak $\lambda$-coding property (hence under the set theoretic assumptions of $3.19, I\left(\lambda^{+}, \mathfrak{K}\right) \geq 2^{\lambda^{+}}$).

4) If $\mathbf{C}$ is local ( $\lambda$-construction framework), $F$ a $\lambda$-amalgamation choice function, with the local $\lambda$-coding property, then $F$ has the $\lambda$-coding property (hence under the set theoretic assumptions of 3.18, we have $I\left(\lambda^{+}, \mathfrak{K}\right) \geq 2^{\lambda^{+}}$and $\left(2^{\lambda}\right)^{+}<2^{\lambda^{+}} \Rightarrow$ $\left.\operatorname{IE}\left(\lambda^{+}, \mathfrak{K}\right) \geq 2^{\lambda^{+}}\right)$.

Remark. The parallel of part (2) holds for local and weaker local property if $F$ acts on sequences. See 3.22 below. 
Proof. 1) Check.

2) Here we use clause $(d)^{-}$rather than $(d)$.

3) Similar (or read the proof of 3.25).

4) Check.

In this context we may consider

3.22 Definition. We say that $F$ is a $\lambda$-amalgamation choice function for sequences, for $\mathbf{C}$ if:

(a) if $x=F\left(x_{1}, x_{2}, x_{3}, x_{4}, x_{5}, x_{6}\right)$ is defined then for some $\alpha_{1}, \alpha_{2}, \alpha_{3}, \alpha<\lambda$ we have $x_{\ell}=\bar{M}^{\ell} \in \mathbf{S} e q_{\alpha_{\ell}}^{s}$ for $\ell<3, \bar{M}^{1} \triangleleft \bar{M}^{2}, t=x_{4}$ is a set of pairwise disjoint intervals $\subseteq \alpha_{i}, \bar{M}^{1}<_{t}^{*} \bar{M}^{3}, A=x_{5}$ a set of $<\lambda$ ordinals $<\lambda^{+}$, $x=\bar{M} \in \mathbf{S} e q_{\alpha}^{s}, M_{\alpha}$ has universe $A, \bar{M}^{1} \leq_{t \cup\left\{\left[\alpha_{1}, \alpha_{2}\right]\right\}}^{*} \bar{M}$

(b) [uniqueness]

as in Definition 3.10.

3.23 Claim. Assume:

(a) $(\exists \mu<\lambda)\left(2^{\mu}=2^{<\mu}<2^{\lambda}\right), 2^{\lambda}<2^{\lambda^{+}}$or at least we have the definable weak diamond for $\lambda$ and $\lambda^{+}$; and $2^{<\lambda} \geq \lambda^{+}$.

(b) $\mathbf{C}$ is a nice ( $\lambda$-construction framework).

(c) $F$ is a $\lambda$-amalgamation choice function for $\mathbf{C}$.

(d) $F$ has the weaker $\lambda$-coding property for $\lambda$.

Then $I\left(\lambda^{+}, \mathfrak{K}\right) \geq \mu_{\mathrm{wd}}\left(\lambda^{+}\right)$.

Proof. Straight forward using 1.4.

Let $\left\langle M_{i}: i\left\langle i^{*}\right\rangle\right.$ list the models in $\mathfrak{K}_{\lambda^{+}}$up to isomorphisms and assume toward contradiction that $i^{*}<\mu_{\mathrm{wd}}\left(\lambda^{+}\right)$. It is enough to choose by induction on $\alpha<\lambda^{+}$a sequence $\bar{M}_{0}^{\eta}$ for $\eta \in{ }^{\alpha} 2$ such that:

(a) $\bar{M}^{\eta} \in \mathbf{S} e q_{\lambda}$

(b) $M_{\lambda}^{\eta}$ has universe $\gamma_{\eta}<\lambda^{+}$

(c) $\nu \triangleleft \eta \Rightarrow \bar{M}^{\nu} \leq_{F} \bar{M}^{\eta}$

(d) if $\delta=\ell g(\eta)$ is a limit ordinal then $\bar{M}^{\eta}$ is a mub of $\left\langle\bar{M}^{\eta\lceil\alpha}: \alpha<\delta\right\rangle$

(e) if $\eta^{\wedge}\langle 0\rangle \unlhd \nu \in \lambda^{\lambda^{+}} 2$ then $M_{\lambda}^{\eta^{\wedge}\langle 1\rangle} \uparrow \tau$ cannot be $\leq_{\mathfrak{K}^{-} \text {-embedded into }}$ $M_{\lambda}^{\nu} \uparrow \tau$ over $M_{\lambda}^{\eta}\lceil\tau$.

This is possible by $3.21(3)$.

Having the $M_{\eta}, \eta \in{ }^{\lambda>} 2$ we get the conclusion by 1.5 .

3.24 Remark. 1) If we are just interested in $I\left(\lambda^{+}, K\right)$ rather than also in $I E\left(\lambda^{+}, K\right)$, then we can change the definition of $\tau$-contradictory to:

$N^{1}, N^{2}$ are $\tau$-contradictory amalgamations of $M_{1}, N$ over $M_{0}$ if $M_{0} \leq_{\mathfrak{K}^{+}}$ $M_{1} \leq_{\mathfrak{K}^{+}} N^{\ell}, M_{0} \leq_{\mathfrak{K}^{+}} N_{0} \leq_{\mathfrak{K}^{+}} N^{\ell}, M_{0}=M_{1} \cap N_{0}$ and there are no $N_{*}^{1}, N_{*}^{2} \in$ $\mathfrak{K}_{<\lambda}^{+}$such that: $N^{\ell} \leq_{\mathfrak{K}^{+}} N_{*}^{\ell}$ and $N_{*}^{1} \uparrow \tau, N_{*}^{2} \uparrow \tau$ are isomorphic over $M_{1} \cup N_{0}$. 
2) Note that it is unreasonable to assume that we will always use the local versions: e.g. if we have a superlimit model in $\mathfrak{K}_{\lambda^{+}}$and we want to have $\bar{M} \in \mathbf{S} e q_{\lambda} \Rightarrow M_{\lambda}$ superlimit, we have to add some global condition (see [Sh 600]). Also possibly we will have in the construction (i.e. in 3.18 or 3.19 ) that $\bar{M}_{\eta}$ has two "contradictory" extensions $\bar{M}_{\eta^{\wedge}\langle 0\rangle}, \bar{M}_{\eta^{\wedge}\langle 1\rangle}$, (see clauses (e) and (f) in their proof) only when $\operatorname{cf}(\ell g(\eta))=\theta$, where $S=\left\{\delta: \delta<\lambda^{+}\right.$and $\left.\operatorname{cf}(\delta)=\theta\right\} \notin \operatorname{WDmId}(\lambda)$; or even $\ell g(\eta) \in S$, for a given $S \in \operatorname{WDmId}(\lambda)^{+}$. We shall deal with such cases when needed.

Remark. We intend to continue this elsewhere.

3.25 Lemma. Let $\mathfrak{K}$ be an abstract elementary class with $L S(\mathfrak{K}) \leq \lambda$ which is categorical in $\lambda$ and in $\lambda^{+}$, with $1 \leq I\left(\lambda^{++}, K\right)<2^{\lambda^{++}}$. Assume that $2^{\lambda}<2^{\lambda^{+}}<$ $2^{\lambda^{++}}$, or at least that the definitional weak diamond holds for both $\lambda^{+}$and $\lambda^{++}$.

If there is a model in $K_{\lambda^{+}}$which is saturated over $\lambda$, then the minimal triples are dense in $K_{\lambda}^{3}$.

Proof. Let $\mathbf{C}$ be $\mathbf{C}_{\mathfrak{K}, \lambda^{+}}^{0}$ (see Definition 3.6) hence $\mathbf{C}$ is explicitly local $\lambda$-construction framework (by 3.7(1)). Suppose toward contradiction that above $\left(M^{*}, N^{*}, a^{*}\right) \in$ $K_{\lambda}^{3}$, there is no minimal triple. We claim in this case that there is a $\lambda^{+}$-amalgamation choice function $F$ for $\mathbf{C}$ with the $\lambda^{+}$-coding property, with domain the quadruples $\left(M_{0}, M_{1}, M_{2}, A, b\right)$ such that: $\left(M^{*}, N^{*}, a\right)$ embeds in $\left(M_{0}, M_{2}, b\right) ; A$ and the universes of $M_{1}, M_{2}$ are contained in $\lambda^{++} ;\left|A \backslash\left(\left|M_{1}\right| \cup\left|M_{2}\right|\right)\right|=\lambda$; and $M_{0} \leq_{\mathfrak{K}} M_{1}, M_{2}$. Then applying 3.17 and 3.18 we get a contradiction.

We first make two observations concerning triples $(M, N, b)$ lying above $\left(M^{*}, N^{*}, b\right)$. Any such triple has the extension property by 2.11 (or just 2.9(1)) and hence we can manufacture a $\lambda^{+}$-amalgamation choice function with the specified domain. Furthermore, there is $M^{\prime} \in K_{\lambda^{+}}$with $M \leq_{\mathfrak{K}} M^{\prime}$ such that $\operatorname{tp}(a, M, N)$ has more than one extension to $M^{\prime}$, by the failure of minimality.

Let us show that any $\lambda^{+}$-amalgamation choice function $F$ with the specified domain has the $\lambda^{+}$-coding property on $\mathbf{C}=\mathbf{C}_{\mathfrak{K}, \lambda^{+}}^{0}$.

Let $\bar{M}^{1} \in \mathbf{S e q}_{\lambda^{+}}$and let $\mathbf{f}^{1}: \lambda \rightarrow \lambda$. For any set $S$ we must find sequences $\bar{M}^{2, \eta}$ (depending on $S$ ) as in 3.21(1). The approach will be to first build suitable $M^{2, \eta}$ for all $\eta \in{ }^{\lambda} 2$, independent of $S$, then restrict appropriately given $S$.

Let $M^{1}=\bigcup_{i} M_{i}^{1}$. Then $M^{1} \in \mathfrak{K}_{\lambda^{+}}$and by our assumptions $M^{1}$ is therefore saturated over $\lambda$. Hence we may suppose $M^{*} \leq_{\mathfrak{K}} M^{1}$. We may also suppose that the universe of $M^{1}$ is an ordinal, and we may choose a subset $A^{2}$ of $\lambda^{++}$ which is the union of an increasing continuous sequence $A_{\alpha}^{2}$ (for $\alpha<\lambda^{+}$) so that: $A_{\alpha}^{2} \cap\left|M^{1}\right|=\left|M_{\alpha}^{1}\right|$ and $A_{0}^{2} \backslash\left|M_{0}^{1}\right|$ and $\left.A_{\alpha+1}^{2} \backslash\left(A_{\alpha}^{2} \cup \mid M_{\alpha+1}^{1}\right)\right)$ have cardinality $\lambda$. Let $E$ be the club:

$$
\left\{\delta<\lambda \text { : for } \alpha<\delta \text {, we have } \alpha+\mathbf{f}^{1}(\alpha)+1<\delta\right\} .
$$

We now define triples $\left(M_{\eta}^{*}, N_{\eta}^{*}, a^{*}\right)$ for all $\eta \in{ }^{i} 2$, by induction on $i$, together with ordinals $\alpha_{\eta}$ satisfying the following conditions:

(a) for any $\eta \in{ }^{\lambda} 2$, the sequence $\left(M_{\eta\lceil j}^{*}, N_{\eta\lceil j}^{*}, a^{*}\right)(j \leq i)$ is increasing and continuous; and similarly the $\alpha_{\eta}$ are increasing and continuous. 
(b) $\left(M_{<>}^{*}, N_{<>}^{*}\right)=\left(M^{*}, N^{*}\right)$.

(c) $M_{\eta}^{*}=M_{\alpha_{\eta}}^{1}$ and the universe of $N_{\eta}^{*}$ is $A_{\alpha_{\eta}}^{2}$.

(d) If $\delta \in E, \eta \in{ }^{\delta} 2$ and $\alpha_{\eta}=\delta$, then:

$(d 1)$ for $i<\mathbf{f}^{1}(\delta)$ and $\eta \triangleleft \nu \in{ }^{\delta+i+1} 2$, the model $N_{\nu}^{*}$ is given by $F$ applied to amalgamate $M_{\delta+i+1}^{1}$ and $N_{\nu \uparrow(\delta+i)}^{*}$ over $M_{\delta+i}^{1}$, using $A_{\delta+i+1}^{2}$ and keeping $a^{*}$ out of $M_{\delta+i+1}^{*}$

$(d 2)$ for all $\nu, \nu^{\prime} \in{ }^{\delta+\mathbf{f}^{1}(\delta)+1} 2$ extending $\eta$ if $\nu^{\prime} \neq \nu$, then for some $\beta$ $\operatorname{tp}\left(a^{*}, M_{\beta}^{*}, N_{\nu}^{*}\right) \neq \operatorname{tp}\left(a^{*}, M_{\beta}^{*}, N_{\nu}^{*}\right)$

(d3) for non-zero $i \leq \mathbf{f}^{1}(\delta)$ and $\nu$ such that $\eta \triangleleft \nu \in{ }^{\delta+i} 2$ we have $M_{\nu}^{*}=M_{\alpha_{\nu}}^{1}$ and $\alpha_{\nu}=\alpha_{\ell g(\nu)}=\alpha_{\eta}+i=\alpha_{\delta}+i\left(\right.$ so $\left.M_{\nu}^{*}=M_{\delta+i}^{1}\right)$

(d4) for non-zero $i \leq \mathbf{f}^{1}(\delta)$ and $\nu, \rho \in{ }^{\delta+i} 2$ such that $\eta \triangleleft \nu \& \eta \triangleleft \rho$ we have $N_{\nu}^{*}=N_{\rho}^{*}$ call it $N_{\eta}^{1}$.

During carrying the definition the main point is guaranteeing clause $(d)$. So let $\eta \in{ }^{\delta_{2}}$ and assume that $\alpha_{\eta}=\delta \in E^{*}$. First we define by induction on $i \leq \mathbf{f}^{1}(\delta)$, a model $N_{\eta, i}^{1}$ such that $N_{i}^{1}$ has universe $A_{\delta+i}^{2}, N_{\eta, i}^{1}$ is $\leq_{\mathfrak{K}}$-increasing, contradiction, $M_{\delta+i}^{1} \leq_{\mathfrak{K}} N_{\eta, i}^{1}$ and $N_{\eta, 0}^{1}=N_{\eta}^{*}$ and $f, i<\mathbf{f}^{1}(\delta)$ then

$$
N_{\eta, i+1}^{1}=F\left(M_{\delta+i}^{1}, M_{\delta+i+1}^{1}, N_{\eta, i}^{1}, A_{\delta+i+1}^{2}, a^{*}\right) .
$$

Next we choose by induction on $i \leq \mathbf{f}^{1}(\delta)$ for each $\rho \in{ }^{i}$, an ordinal $\beta_{\eta, \ell} \in$ $\left[\delta+\mathbf{f}^{1}(\delta), \lambda^{+}\right)$and model $N_{\eta, \rho}^{1}$ such that:

(i) $N_{\eta, \rho}^{1} \in K_{\lambda}$ has universe $A_{\beta_{\eta, \rho}}^{2}$

(ii) $M_{\beta_{\eta, \rho}}^{1} \leq_{\mathfrak{K}} N_{\eta, \rho}^{1}$ and $\left(a^{*} /={ }^{N_{\eta, \rho}^{1}}\right) \notin M_{\beta_{\eta, \rho}}^{1} /={ }^{N_{\eta, \rho}^{1}}$

(iii) $\beta_{\eta,<>}=\delta+\mathbf{f}^{1}(\delta)$

(iv) $\rho_{1} \triangleleft \rho_{2} \Rightarrow \beta_{\eta, \rho_{1}}<\beta_{\eta, \rho_{2}} \& N_{\eta, \rho_{1}}^{1} \leq_{\mathfrak{K}} N_{\eta, \rho_{2}}^{1}$

(v) $i$ limit $\Rightarrow N_{\eta, \rho}^{1}=\bigcup_{\zeta<i} N_{\eta, \rho \uparrow \zeta}^{1}$

(vi) $\beta_{\eta, \rho^{\wedge}<0>}=\beta_{\eta^{\wedge} \rho^{\wedge}<1>}$ and $\operatorname{tp}\left(a^{*}, M_{\beta_{\eta, \rho^{\wedge}<0>}}^{1}, N_{\eta, \rho^{\wedge}<0>}^{1}\right) \neq \operatorname{tp}\left(a^{*}, M_{\beta_{\eta, \rho^{\wedge}<1>}}^{1}, N_{\eta, \rho^{\wedge}<1>}^{1}\right)$.

There is no problem to carry the definition. Now let

$$
\begin{aligned}
& \alpha_{\eta^{\wedge} \rho}=\delta+i=\ell g\left(\eta^{\wedge} \rho\right) \text { if } \rho \in{ }^{i} 2 \text { and } i \leq \mathbf{f}^{1}(\delta) \\
& \alpha_{\eta^{\wedge} \rho}=\beta_{\eta, \rho} \text { if } \rho \in \mathbf{f}^{1}(\delta)+12 \\
& M_{\eta^{\wedge} \rho}^{*}=M_{\alpha_{\eta^{\wedge} \rho}}^{1} \text { if } \rho \in{ }^{i} 2, i \leq \mathbf{f}^{1}(\delta)+1 \\
& N_{\eta^{\wedge} \rho}^{*}=N_{\eta, i}^{1} \text { if } \rho \in{ }^{i} 2 \text { and } i \leq \mathbf{f}^{1}(\delta)
\end{aligned}
$$




$$
N_{\eta^{\wedge} \rho}^{*}=N_{\eta, \rho}^{1} \text { if } \rho \in \mathbf{f}^{1}(\delta)+12 .
$$

Now check.

Having carried the induction for $\eta \in{ }^{\lambda^{+}} 2$ we let $\bar{M}^{2, \eta}=\left\langle N_{\eta \mid \alpha}^{*}: \alpha\left\langle\lambda^{+}\right\rangle\right.$and $\mathbf{f}^{2}=\mathbf{f}^{1}+1$. We have to check that the demand in Definition 3.14 holds.

Note that this is essentially the proof mentioned in 3.16(9).

By our initial remarks there is little difficulty in carrying out this induction.

We then set $\bar{M}^{2, \eta}=\left\langle N_{\eta \uparrow i}^{*}: i\left\langle\lambda^{+}\right\rangle\right.$for $\eta \in{ }^{\lambda^{+}} 2$. Given a set $S \subseteq \lambda^{+}$we consider $\bar{M}^{2, \eta}$ for $\eta \in{ }^{\lambda^{+}} 2$ extending $0_{\lambda \backslash S}$, together with the function $\mathbf{f}^{2}$ equal to $\mathbf{f}^{1}+1$ on $S$ and to $\mathbf{f}^{1}$ on $S$. We claim that the two conditions of Definition 3.21(1) are met.

The first of these is a condition on the type of construction allowed, and, of course, it has been obeyed, notably in $(d 1)$ above:

$(*)_{1}\left(\bar{M}^{1}, \mathbf{f}^{1}\right) \leq_{F, a}^{\text {at }}\left(\bar{M}^{2, \eta}, \mathbf{f}^{1}\right)$; and $M^{2, \eta}\lceil\alpha$ is determined by $\eta\lceil\alpha$.

The second condition referred to a club $E$, which can be the intersection of the club we have defined above with $\left\{\delta: \alpha_{\delta}=\delta\right\}$. This condition goes as follows:

$(*)_{2}$ it is impossible to find sequences $\eta^{3}, \eta^{4}$ (extending $0_{\lambda \backslash S}$ ), extensions $\left(\bar{M}^{1}, \mathbf{f}^{2}\right) \leq_{F}$ $\left(\bar{M}^{3}, \mathbf{f}^{3}\right),\left(\bar{M}^{4}, \mathbf{f}^{4}\right)$ witnessed by clubs $E^{3}, E^{4}$ (i.e. $E^{\ell}$ is the intersection of the clubs which witness the atomic relations $\leq_{F}^{\text {at }}$ implicit in $\leq_{F}$ ), and embeddings $f_{\ell}: M^{2, \eta^{\ell}} \rightarrow M^{\ell}(\ell=3$ or 4$)$ over $M^{1}$ such that for some $\delta \in E \cap E^{3} \cap E^{4} \cap S$ we have:

(i) $\bar{M}^{3} \uparrow\left(\delta+\mathbf{f}^{2}(\delta)+1\right)=\bar{M}^{4} \uparrow\left(\delta+\mathbf{f}^{2}(\delta)+1\right) ;$

(ii) $\mathbf{f}^{3} \uparrow \delta=\mathbf{f}^{4} \uparrow \delta$;

(iii) $\eta^{3}\left\lceil\delta=\eta^{4}\left\lceil\delta\right.\right.$ (call the restriction $\eta$ ) and $\eta^{3}(\delta) \neq \eta^{4}(\delta)$;

(iv) $f_{3}, f_{4}$ are equal on $M_{\delta}^{2, \eta}$; and

(v) for $\ell=3,4, f_{\ell} \operatorname{maps} M_{\delta}^{2, \eta}$ into $M_{\delta}^{\ell}$.

Suppose on the contrary we have $\eta^{3}, \eta^{4}$ (extending $\left.0_{\lambda \backslash S}\right),\left(\bar{M}^{1}, \mathbf{f}^{2}\right) \leq_{F}\left(\bar{M}^{3}, \mathbf{f}^{3}\right)$, $\left(\bar{M}^{4}, \mathbf{f}^{4}\right), E^{3}, E^{4}, f_{3}, f_{4}$ and $\delta$ as above.

Let $\hat{M}=M_{\delta+\mathbf{f}^{2}(\delta)}^{3}$. It follows from condition (i) and the fact that $\delta$ belongs to the witnessing clubs $E^{3}, E^{4}$ that $\hat{M}=M_{\delta+\mathbf{f}^{2}(\delta)}^{4}$. Then $f_{3}, f_{4}$ provide embeddings of $N_{\eta^{3} \uparrow\left(\delta+\mathbf{f}^{1}(\delta)+1\right)}^{*}$ and $N_{\eta^{3} \uparrow\left(\delta+\mathbf{f}^{1}(\delta)+1\right)}^{*}$ into $\hat{M}$ which agrees on $N_{\eta}^{*}$ (hence on $a^{*}$ ) and on $M_{\eta}^{1}$. By $3.16(2)$ we are done.

3.26 Lemma. Let $\mathfrak{K}$ be an abstract elementary class with $L S(\mathfrak{K}) \leq \lambda$ which is categorical in $\lambda$ and in $\lambda^{+}$, with $1 \leq I\left(\lambda^{++}, K\right)<2^{\lambda^{++}}$. Assume that $2^{\lambda}<2^{\lambda^{+}}<$ $2^{\lambda^{++}}$, or at least that the definitional weak diamond holds for both $\lambda^{+}$and $\lambda^{++}$. Then:

(*) for any $M \in K_{\lambda^{+}}$and any triple $\left(M^{0}, N^{0}, a^{0}\right)$ in $K_{\lambda}^{3}$ with $M^{0} \leq_{\mathfrak{K}} M$, we can find sequences $\bar{M}=\left\langle M_{i}: i<\lambda^{+}\right\rangle, \bar{N}=\left\langle N_{i}: i<\lambda^{+}\right\rangle$such that 
(a) $\left(M^{0}, N^{0}, a^{0}\right)=\left(M_{0}, N_{0}, a^{0}\right)$;

(b) $\left(M_{i}, N_{i}, a\right)$ is increasing and continuous in $K_{\lambda}^{3}$;

(c) the union of the $M_{i}$ is $M$;

(d) the set $S(\bar{M}, \bar{N}, a)$ of $\delta<\lambda^{+}$such that for some $j>\delta$ for all $i \geq j$

if we have $\left(M_{j}, N_{j}, a\right) \leq_{\ell}\left(M_{i}, N^{\ell}, a\right)$ for $\ell=1,2$ then we can amalgamate $\left(M_{i}, N^{1}, a\right)$ and $\left(M_{i}, N^{2}, a\right)$ over $\left(M_{i}, N_{\delta}, a\right)$

is stationary in $\lambda^{+}$.

Proof. Otherwise, we claim that any full $\lambda^{+}$-amalgamation choice function will have the $\lambda^{+}$-coding property.

Let $\bar{M} \in \mathbf{S e q}_{\lambda^{+}}, \mathbf{f}: \lambda^{+} \rightarrow \lambda^{+}$and $S \subseteq \lambda^{+}$be given. Then as $K$ is categorical in $\lambda^{+}$, we may suppose that $M$ is the union of the $M_{i}$. If (c) fails, then (d) fails on a club of $\delta$, providing enough failures of amalgamation to carry the proof as in 3.12. $\square_{3.12}$

3.27 Lemma. Let $\mathfrak{K}$ be an abstract elementary class with $L S(\mathfrak{K}) \leq \lambda$ which is categorical in $\lambda, \lambda^{+}$and $\lambda^{++}$and with no model in cardinality $\lambda^{+3}$.

Suppose that there is no model in $K_{\lambda^{+}}$saturated above $\lambda$ and that:

$\left(*^{\prime}\right)$ for any $M \in K_{\lambda^{+}}$and any triple $\left(M^{0}, N^{0}, a^{0}\right)$ in $K_{\lambda}^{3}$ with $M^{0} \leq_{\mathfrak{K}} M$, we can find sequences $\bar{M}=\left\langle M_{i}: i<\lambda^{+}\right\rangle, \bar{N}=\left\langle N_{i}: i<\lambda^{+}\right\rangle$such that

(a) $\left(M^{0}, N^{0}, a^{0}\right)=\left(M_{0}, N_{0}, a^{0}\right)$;

(b) $\left(M_{i}, N_{i}, a\right)$ is increasing and continuous in $K_{\lambda}^{3}$;

(c) the union of the $M_{i}$ is $M$;

(d) the set $S(\bar{M}, \bar{N}, a)$ of $\delta<\lambda^{+}$such that for some $j>\delta$ for all $i \geq j$

if we have $\left(M_{j}, N_{j}, a\right) \leq_{h_{\ell}}\left(M_{i}, N^{\ell}, a\right)$ for $\ell=1,2$ then we can amalgamate $\left(M_{i}, N^{1}, a\right)$ and $\left(M_{i}, N^{2}, a\right)$ over $\left(M_{i}, N_{\delta}, a\right)$

is stationary in $\lambda^{+}$.

$\underline{\text { Then }}$ the minimal triples are dense in $K_{\lambda}^{3}$.

Proof. Suppose that there is no minimal triple above $\left(M^{*}, N^{*}, a^{*}\right)$. It suffices to show that there is no maximal model in $K_{\lambda^{++}}$and as $K_{\lambda^{++}}$is categorical, this will follow from the existence of a single pair of models $\left(M^{\prime}, N^{\prime}\right)$ in $K_{\lambda^{+}+}$with $M^{\prime}<_{\mathfrak{K}} N^{\prime}$. So it suffices to show:

every triple $(M, N, a)$ in $K_{\lambda^{+}}^{3}$ has a proper extension in $K_{\lambda^{+}}^{3}$

as the desired pair $\left(M^{\prime}, N^{\prime}\right)$ can then be built as the limit of an increasing continuous chain. 
Fix $(M, N, a)$ in $K_{\lambda^{+}}^{3}$. As there is no model saturated over $\lambda$ in $K_{\lambda^{+}}$, there is some $M_{0}$ in $K_{\lambda}$ over which there are more than $\lambda^{+}$types. By $\lambda$-categoricity we may suppose $M_{0} \leq_{\mathfrak{K}} M$. Fix a triple $\left(M_{0}, N_{0}, b\right)$ in $K_{\lambda}^{3}$ for which $\operatorname{tp}\left(b, M_{0}, N_{0}\right)$ is not realized in $M$.

Apply $\left(*^{\prime}\right)$ to $M$ and $\left(M_{0}, N_{0}, b\right)$ to get sequences $\bar{M}, \bar{N}$ of length $\lambda^{+}$as in $\left(*^{\prime}\right)$. Let $S=S(\bar{M}, \bar{N}, b)$. In particular $M=\bigcup M_{i}$. Let $M^{1}=\bigcup N_{i}$. As $\mathfrak{K}$ has amalgamation in $\lambda^{+}$we may suppose $M^{1}, N \leq_{\mathfrak{K}} N^{1}$ with $N^{1} \in \mathfrak{K}_{\lambda^{+}}$.

We can also choose an increasing continuous sequence $\left(M_{i}^{\prime}, N_{i}^{\prime}, a^{*}\right)$ beginning with $\left(M^{*}, N^{*}, a^{*}\right)$ such that each $\left(M_{i}^{\prime}, N_{i}^{\prime}, a^{*}\right)$ is reduced and $\operatorname{tp}\left(a^{*}, M_{i}^{\prime}, N_{i}^{\prime}\right)$ has more than one extension in $\mathscr{S}\left(M_{i+1}^{\prime}\right)$, using the failure of minimality and 2.7(1). By categoricity we may suppose $M=\bigcup M_{i}^{\prime}$. Set $M^{2}=\bigcup N_{i}^{\prime}$. By amalgamation we may suppose $M^{2}, N^{1} \leq_{\mathfrak{K}} N^{2} \in K_{\lambda^{+}}$.

We claim that one of the triples $\left(M^{1}, N^{1}, a\right)$ or $\left(M^{2}, N^{2}, a\right)$ is a proper extension of $(M, N, a)$. Suppose on the contrary that $a$ belongs to both $M^{1}$ and $M^{2}$.

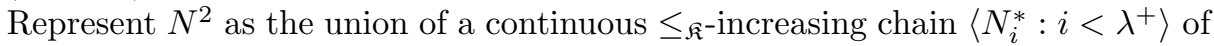
models in $K_{\lambda}$.

Let $E$ be

$$
\left\{i<\lambda^{+}: M_{i}=M_{i}^{\prime} ; N_{i}^{*} \cap M=M_{i} ; N_{i}^{*} \cap M^{1}=N_{i} ; N_{i}^{*} \cap M^{2}=N_{i}^{\prime}\right\},
$$

a club in $\lambda^{+}$.

Fix $\delta \in E \cap S$ such that $a$ is in $N_{\delta}$ and $N_{\delta}^{\prime}$. We show now that $a^{*} \in N_{\delta}$. Now $\left(M_{\delta}^{\prime}, N_{\delta}^{\prime}, a^{*}\right)$ is reduced. If $a^{*} \notin N_{\delta}$ then $\left(N_{\delta}, N^{2}, a^{*}\right)$ lies over $\left(M_{\delta}^{\prime}, N_{\delta}^{\prime}, a^{*}\right)$ and hence $N_{\delta} \cap N_{\delta}^{\prime} \subseteq M_{\delta}^{\prime}$; but the element $a$ witnesses the failure of this condition. So $a^{*} \in N_{\delta}$.

Let $j>\delta$ be chosen in accordance with the definition of $S(\bar{M}, \bar{N}, b)$ and let $i>j^{\prime}>j_{a}, k, j^{\prime} \in E$. As tp $\left(a^{*}, M_{j^{\prime}}^{\prime}, N_{j^{\prime}}^{\prime}\right)$ has more than one extension to $M_{j^{\prime}+1}^{\prime}$, the same applies to $M_{i}^{\prime}$. However, $M_{i}^{\prime}=M_{i}$ and $M_{j^{\prime}}^{\prime}=M_{j^{\prime}}$ and thus $\operatorname{tp}\left(a^{*}, M_{j^{\prime}}, N_{j^{\prime}}^{2}\right)=$ $\operatorname{tp}\left(a^{*}, M_{j^{\prime}}, N_{j^{\prime}}^{*}\right)=\operatorname{tp}\left(a^{*}, M_{j^{\prime}}, N_{j^{\prime}}\right)$ has more than one extension over $M_{i}$. Thus, $M_{i}$ and $N_{j^{\prime}}$ may be amalgamated in two incompatible ways over $M_{j^{\prime}}$, getting $N^{+}$ and $N^{-}$, say (i.e. the $N^{+}$and $N^{-}$cannot be amalgamated over $M_{i}$ preserving the images of $\left.a^{*}\right)$. Furthermore $\left(M_{i}, N^{ \pm}, b\right)$ lies above $\left(M_{j}, N_{j}, b\right)$ in $K_{\lambda^{+}}^{3}$, that is, $b$ is not mapped into $M_{i}$, because $M$ does not realize $\operatorname{tp}\left(b, M_{0}, N_{0}\right)$. However, this contradicts the definition of $S$, as the triples $\left(M_{i}, N^{+}, b\right)$ and $\left(M_{i}, N^{-}, b\right)$; cannot be amalgamated over $\left(M_{i}, N_{\delta}\right)$ since $a^{*}$ belongs to $N_{\delta}$.

3.28 Theorem. Let $\mathfrak{K}$ be an abstract elementary class with $L S(\mathfrak{K}) \leq \lambda$ which is categorical in $\lambda$ and in $\lambda^{+}$with $1 \leq I\left(\lambda^{++}, K\right)<2^{\lambda^{++}}$. Assume that $2^{\lambda}<2^{\lambda^{+}}<$ $2^{\lambda^{++}}$, or at least that the definitional weak diamond holds for both $\lambda^{+}$and $\lambda^{++}$.

Then under either of the following assumptions, the minimal triples are dense in $K_{\lambda}^{3}$ :

(A) $K$ is categorical in $\lambda^{++}$and has no model in cardinality $\lambda^{+3}$;

(B) there is a model saturated above $\lambda$ in cardinality $\lambda^{+}$.

Proof. By the previous lemmas 3.25, 3.26, 3.27. Note that $\left(*^{\prime}\right)$ is exactly the negation of $(*)$. 
3.29 Remark. This will be proved without the additional assumptions $(A, B)$ in [Sh 600]. In any case this does not affect the proof of Theorem 0.5

3.30 Claim. Let $\mathfrak{K}$ be an abstract elementary class with $L S(\mathfrak{K}) \leq \lambda$ which is categorical in $\lambda$ and in $\lambda^{+}$, with $1 \leq I\left(\lambda^{++}, K\right)<2^{\lambda^{++}}$, and with no model in cardinality $\lambda^{+3}$. Assume that $2^{\lambda}<2^{\lambda^{+}}<2^{\lambda^{++}}$.

Then the minimal triples are dense in $K_{\lambda}^{3}$.

Proof. If $2^{\lambda^{+}}>\lambda^{++}$we get the conclusion by 2.7. If $2^{\lambda^{+}}=\lambda^{++}$then as $2^{\lambda}<2^{\lambda^{+}}$ we have $2^{\lambda}=\lambda^{+}$. Thus, there is a model in $K_{\lambda^{+}}$which is saturated above $\lambda$, and Lemma 3.28 applies. 


\section{$\S 4$ Minimal types}

We return to the analysis of minimal types initiated in 2.12 .

We use from $\S 2$ only $2.1,2.6,2.9$ so there are repetitions.

\subsection{Hypothesis.}

(a) $\mathfrak{K}$ is an abstract elementary class with $L S(\mathfrak{K}) \leq \lambda$ (for simplicity $K_{<\lambda}=\emptyset$ ).

(b) $\mathfrak{K}$ is categorical in $\lambda, \lambda^{+}$with $K_{\lambda+2} \neq \emptyset$ (note: $(a)+(b)=(*)_{\lambda}^{3}$ of 2.4 ).

(c) $\mathfrak{K}$ has amalgamation in $\lambda(2.2(1))$, so by $(a)+(c)$, we have $(*)_{\lambda}^{2}$ from 2.9 hence $\mathfrak{K}$ satisfies the model theoretic properties which were deduced in 2.4-2.6,2.9 in particular:

(i) every $(M, N, a) \in K_{\lambda}^{3}$ has the weak extension property 2.4 ;

(ii) criteria for the extension property 2.9 ;

(iii) basic Definitions and properties 2.3, 2.6.

4.2 Definition. 1) If $p \in \mathscr{S}(N), N \in K_{\lambda}$ and $N^{\prime} \in K_{\lambda}$ remember (from Definition $2.13)$

$$
\mathscr{S}_{p}\left(N^{\prime}\right)=\left\{f(p): f \text { is an isomorphism from } N \text { onto } N^{\prime}\right\}
$$

and let

$$
\begin{aligned}
\mathscr{S}_{\geq p}\left(N^{\prime}\right)=\left\{q \in \mathscr{S}\left(N^{\prime}\right):\right. & q \text { not algebraic (i.e. not realized by any } \\
& \left.c \in N^{\prime}\right) \text { and, for some } N^{\prime \prime} \in K_{\lambda}, \\
& N^{\prime \prime} \leq_{\mathfrak{K}} N^{\prime}, \text { we have } q\left\lceil N^{\prime \prime} \in \mathscr{S}_{p}\left(N^{\prime \prime}\right)\right\} .
\end{aligned}
$$

2) We say the type $p \in \mathscr{S}(N)$ is $\lambda$-algebraic if $\|N\| \leq \lambda$ and for every $M$ such that $N \leq_{\mathfrak{K}} M$ we have: $\lambda \geq|\{c \in M: \operatorname{tp}(c, N, M)=p\}|$.

4.3 Claim. If $\left(M_{0}, M_{1}, a\right) \in K_{\lambda}^{3}$ is minimal, then it has the extension property.

Proof. Let $p^{*}=\operatorname{tp}\left(a, M_{0}, M_{1}\right)$, and assume it is a counter-example. We note:

$\bigotimes_{1}$ for some $M^{*}$ we have $M_{0} \leq_{\mathfrak{K}} M^{*} \in K_{\lambda}$ but for no $M^{+}$and $b$ do we have $M^{*} \leq_{\mathfrak{K}} M^{+} \in K_{\lambda}, b \in M^{+} \backslash M^{*}$ and $b$ realizes $p^{*}$.

[Why? If not for every $N, M_{0} \leq_{\mathfrak{K}} N \in K_{\lambda}$, we can find $N_{1}$, $N \leq_{\mathfrak{K}} N_{1} \in K_{\lambda}$ and $b \in N_{1} \backslash N$ which realizes $p^{*}$. Hence (as $\mathfrak{K}_{\lambda}$ has amalgamation in $\lambda$ ) we can find $N_{2}$ such that $N_{1} \leq_{\mathfrak{K}} N_{2} \in K_{\lambda}$, and $g$ a $\leq_{\mathfrak{K}}$-embedding of $M_{1}$ into $N_{2}$ extending $\operatorname{id}_{M_{0}}$ such that $g(a)=b$.

This proves the extension property]. 
$\bigotimes_{2}$ if $p \in \mathscr{S}_{\geq p^{*}}(N)$ and $N \in K_{\lambda}$ and $N \leq_{\mathfrak{K}} N^{*} \in K$, then the set of elements of $b \in N^{*}$ realizing $p$ has cardinality $\leq \lambda$.

[Why? by 4.1(c)(ii); so indirectly $2.9(2)]$.

$\bigotimes_{3}$ if $N \in K_{\lambda}$, then $\left|\mathscr{S}_{\geq p^{*}}(N)\right|>\lambda^{+}$.

Proof of $\otimes_{3}$. If $N$ forms a counterexample, as $K$ is categorical in $\lambda$ and using $\otimes_{2}$ we can find $\left\langle N_{i}: i\left\langle\lambda^{+}\right\rangle, \leq_{\mathfrak{K}^{-}}\right.$increasing continuous sequence of members of $K_{\lambda}$ such that:

(*) for every $\alpha<\lambda^{+}$and $q \in \mathscr{S}_{\geq p^{*}}\left(N_{\alpha}\right)$, for some $\beta=\beta_{q}<\lambda^{+}$we have: for no $N^{\prime}, b$ do we have $N_{\beta} \leq_{\mathfrak{K}} N^{\prime} \in K_{\lambda}, b \in N^{\prime} \backslash N_{\beta}$ and $b$ realizes $q$.

So $N_{\lambda^{+}}=\bigcup_{i<\lambda^{+}} N_{i}$ has the property

(**) if $\bar{N}^{\prime}=\left\langle N_{\alpha}^{\prime}: \alpha<\lambda^{+}\right\rangle$is a representation of $N_{\lambda^{+}}$then for a club of $\delta<\lambda^{+}$ for every $q \in \mathscr{S}_{\geq p^{*}}\left(N_{\delta}^{\prime}\right)$ for a club of $\beta \in\left(\delta, \lambda^{+}\right)$, for no $N^{\prime}, b$ do we have: $N_{\beta} \leq_{\mathfrak{K}} N^{\prime} \in K_{\lambda}, b \in N^{\prime} \backslash N_{\beta}$ and $b$ realizes $q$.

On the other hand, we can choose by induction on $\alpha<\lambda^{+}$a triple $\left(N_{0, \alpha}, N_{1, \alpha}, a\right) \in K_{\lambda}^{3}$ increasing continuous in $\alpha$ such that $\left(N_{00}, N_{10}, \alpha\right)=\left(M_{0}, M_{1}, a\right)$ and $N_{0, \alpha} \neq N_{0, \alpha+1}$ (existence by the weak extension property; i.e. $2.4=4.1(\mathrm{c})(\mathrm{i}))$.

Now $N_{0, \lambda^{+}}=\bigcup_{\alpha<\lambda^{+}} N_{0, \alpha} \in K_{\lambda^{+}}$does not satisfy the statement $(* *)$ :

$\left\langle N_{0, \alpha}: \alpha<\lambda^{+}\right\rangle$is a representation of $N_{0, \lambda^{+}}$, and for every

$\alpha, \operatorname{tp}\left(a, N_{0, \alpha}, N_{1, \alpha}\right)$ extend $\operatorname{tp}\left(a, N_{0,0}, N_{1,0}\right)=\operatorname{tp}\left(a, M_{0}, M_{1}\right)=p^{*}$ hence

$\operatorname{tp}\left(a, N_{0, \alpha}, N_{1, \alpha}\right) \in \mathscr{S}_{\geq p^{*}}\left(N_{0, \alpha}\right)$ satisfies: for every $\beta \in\left(\alpha, \lambda^{+}\right)$, there is $N^{\prime}, N_{0, \beta} \leq_{\mathfrak{K}}$ $N^{\prime}$ and some $b \in N^{\prime} \backslash N_{0, \beta}$ realizes $\operatorname{tp}\left(a, N_{0, \alpha}, N_{1, \alpha}\right)$; simply choose $\left(N^{\prime}, b\right)=$ $\left(N_{1, \beta}, a\right)$. So $N_{0, \lambda^{+}}, N_{\lambda^{+}}$cannot be isomorphic (as one satisfies $(* *)$ the other not). But both are in $K_{\lambda^{+}}$, contradicting the categoricity of $\mathfrak{K}$ in $\lambda^{+}$.

To finish the proof of 4.3 it is enough to prove

4.4 Claim. If $p^{*} \in \mathscr{S}\left(M_{0}\right)$ is minimal, $M_{0} \in K_{\lambda}$, then $N \in K_{\lambda} \Rightarrow\left|\mathscr{S}_{\geq p^{*}}(N)\right| \leq$ $\lambda^{+}$.

Proof. By 4.6, 4.7 below. Note that $\mathscr{S}_{\geq p^{*}}(N)$ has the same cardinality for every $N \in K_{\lambda}$.

4.5 Claim. 1) If $N_{1} \leq_{\mathfrak{K}} N_{2}$ are in $K_{\lambda}$ and $p_{1} \in \mathscr{S}\left(N_{1}\right)$ is minimal and is omitted by $N_{2}$ then $p_{1}$ has a unique extension in $\mathscr{S}\left(N_{2}\right)$, call it $p_{2}$, and

$p_{1} \in \mathscr{S}_{\geq p^{*}}\left(N_{1}\right) \Rightarrow\left[p_{2} \in \mathscr{S}_{\geq p^{*}}\left(N_{2}\right)\right.$ and $p_{2}$ is minimal $]$.

2) If $N_{1} \leq_{\mathfrak{K}} N_{2}$ are in $K_{\lambda}, p_{1} \in \mathscr{S}\left(N_{1}\right)$ minimal, then $p_{1}$ has a unique non-algebraic extension in $\mathscr{S}\left(N_{2}\right)$ called $p_{2}$, it is minimal and $p_{1} \in \mathscr{S}_{\geq p^{*}}\left(N_{1}\right) \Rightarrow p_{2} \in \mathscr{S}_{\geq p^{*}}\left(N_{2}\right)$. 3) (Continuity) if $\left\langle N_{i}: i \leq \alpha\right\rangle$ is a $\leq_{\mathfrak{K}}$-increasing continuous sequence of members 
of $K_{\lambda}, p_{i} \in \mathscr{S}\left(N_{i}\right), p_{0}$ minimal, $p_{i} \in \mathscr{S}\left(N_{i}\right)$ extends $p_{0}$ and is non-algebraic then $\left\langle p_{i}: i \leq \alpha\right\rangle$ is increasing continuously.

Proof of 4.5. Easy. E.g.,

3) If $i<j \leq \alpha$ then $p_{j} \uparrow N_{i}$ is well defined, it belongs to $\mathscr{S}\left(N_{i}\right)$, also it is nonalgebraic and extending $p_{0}$ hence by the uniqueness $(=4.5(1))$ we have $p_{i}=p_{j}\left\lceil N_{i}\right.$. If $\delta \leq \alpha, p_{\delta} \in \mathscr{S}\left(N_{\delta}\right)$ extends $p_{i}$ for $i<\delta$; if $p_{\delta}^{\prime} \in \mathscr{S}\left(N_{\delta}\right)$ extends each $p_{i}(i<\delta)$ then it extends $p_{0}$ and is non-algebraic hence by uniqueness $p_{\delta}^{\prime}=p_{\delta}$.

4.6 Claim. If $N \in K_{\lambda}, \mathscr{S} \subseteq \mathscr{S}(N)$ and $|\mathscr{S}|>\lambda^{+}$, then we can find $N^{*}, N_{i}$ in $K_{\lambda}$, (for $\left.i<\lambda^{++}\right)$such that:

( $\alpha) N \leq_{\mathfrak{K}} N^{*}<_{\mathfrak{K}} N_{i}$

( $\beta$ ) for no $i_{0}<i_{1}<\lambda^{++}$and $c_{\ell} \in N_{i_{\ell}} \backslash N^{*}$ (for $\left.\ell=0,1\right)$ do we have $t p\left(c_{0}, N^{*}, N_{i_{0}}\right)=t p\left(c_{1}, N^{*}, N_{i_{1}}\right)$

$(\gamma)$ there are $a_{i} \in N_{i}\left(\right.$ for $\left.i<\lambda^{++}\right)$such that $t p\left(a_{i}, N, N_{i}\right) \in \mathscr{S}$ (and they are pairwise distinct).

Remark. We use here less than Hypothesis 4.1:

$(*) \mathfrak{K}$ is abstract elementary class with amalgamation in $\lambda$, categorical in $\lambda$, $K_{\lambda^{+}} \neq \emptyset$.

The same applies to 4.7 .

Proof. Without loss of generality $|N|=\lambda$; now choose by induction on $\alpha<$ $\lambda^{++}, \bar{N}^{\alpha}, N_{\alpha}, a_{\alpha}$ such that:

(A) $N_{\alpha} \in K_{\lambda^{+}}$has a set of elements $\lambda \times(1+\alpha)$ and $N_{\alpha}$ is $\leq_{\mathfrak{K}^{-}}$-increasing continuous in $\alpha$

(B) $\bar{N}^{\alpha}=\left\langle N_{i}^{\alpha}: i<\lambda^{+}\right\rangle$is a representation of $N_{\alpha}$ (i.e. is $\leq_{\mathfrak{K}^{-} \text {-increasing }}$ continuous, $\left\|N_{i}^{\alpha}\right\| \leq \lambda$ and $\left.N_{\alpha}=\bigcup_{i<\lambda^{+}} N_{i}^{\alpha}\right)$

(C) for $\alpha<\lambda^{++}$successor, if $i<j<\lambda^{+}, p \in \mathscr{S}\left(N_{i}^{\alpha}\right)$ is realized in $N_{j}^{\alpha}$ and is $\lambda$-algebraic (see Definition 4.2(2)) then for no $N^{\prime}, b$ do we have $N_{j}^{\alpha} \leq_{\mathfrak{K}}$ $N^{\prime} \in K_{\lambda}$ and $b \in N^{\prime} \backslash N_{j}^{\alpha}$ realizes $p$ (actually not needed)

(D) $N=N_{0}^{0} \leq_{\mathfrak{K}} N_{0}$ and $a_{\alpha} \in N_{\alpha+1} \backslash N_{\alpha}$ realizes some $p_{\alpha} \in \mathscr{S}$ not realized in $N_{\alpha}$

(E) If $\aleph_{0}<\operatorname{cf}(\alpha) \leq \lambda \underline{\text { then }}$ let for $j<\lambda$ : $M_{j}^{\alpha}=\cap\left\{\bigcup_{\beta \in C} N_{j}^{\beta}: C\right.$ a club of $\left.\alpha\right\}$ whenever possible, i.e. the result is in $K_{\lambda}$ and $\leq_{\mathfrak{K}} N^{\alpha}$

$(F)$ for each $\alpha<\lambda^{++}$, for a club $E_{\alpha}^{0}$ of ordinals $i<\lambda^{+}$we have $\left(N_{i}^{\alpha}, N_{i}^{\alpha+1}, a_{\alpha}\right)$ is reduced; hence $\left(\operatorname{as} \operatorname{tp}\left(a_{\alpha}, N_{i}^{\alpha}, N_{i}^{\alpha+1}\right)\right.$ extends $\operatorname{tp}\left(a_{\alpha}, N, N_{i}^{\alpha+1}\right)$ which is not realized in $N_{\alpha}$ ): 
(G) for every ( $i \in E_{\alpha}^{0}$ and) $b \in N_{i}^{\alpha+1} \backslash N_{i}^{\alpha}$ the type $\operatorname{tp}\left(b, N_{i}^{\alpha}, N_{i}^{\alpha+1}\right)$ is not realized in $N_{\alpha}$

(a key point).

There is no problem to carry out the construction ( $\mathfrak{K}$ has amalgamation in $\lambda$ ).

Let $w_{i}^{\alpha}=:\left\{\beta: N_{i}^{\alpha+1} \cap N_{\beta+1} \nsubseteq N_{\beta}\right\}$, so necessarily $\left|w_{i}^{\alpha}\right| \leq \lambda$, $w_{i}^{\alpha}$ is increasing continuous in $i<\lambda^{+}$and $\alpha=\bigcup_{i<\lambda^{+}} w_{i}^{\alpha}$ and for $\beta<\alpha$ let

$\mathbf{i}(\beta, \alpha)=\operatorname{Min}\left\{i: \beta \in w_{i}^{\alpha}\right\}$.

Now for every $\alpha \in S^{*}=:\left\{\delta<\lambda^{++}: \operatorname{cf}(\delta)=\lambda^{+}\right\}$, the set

$$
\begin{aligned}
E_{\alpha}=:\left\{i<\lambda^{+}:\right. & i \text { limit }, N \leq_{\mathfrak{K}} N_{i}^{\alpha+1}, a_{\alpha} \in N_{i}^{\alpha+1}, \\
& \text { and for every } \beta<\alpha \text { if } \\
& \beta \in w_{i}^{\alpha} \text { then } \\
& N_{i}^{\beta}=N_{i}^{\alpha} \cap N_{\beta} \text { and for } j<i \\
& \text { the closure of } w_{j}^{\alpha}(\text { in } \alpha) \text { is included in } w_{i}^{\alpha} \\
& \text { and } \left.\beta_{1}<\beta_{2} \& \beta_{1} \in w_{j}^{\alpha} \& \beta_{2} \in w_{j}^{\alpha} \Rightarrow \mathbf{i}\left(\beta_{1}, \beta_{2}\right)<i\right\}
\end{aligned}
$$

is a club of $\lambda^{+}$.

As we can assume $\lambda>\aleph_{0}$ (ignoring $\lambda=\aleph_{0}$ as was treated earlier in [Sh 88] though for a $\mathrm{PC}_{\aleph_{0}}$ class, or see $[$ Sh 600$\left.], \S 2\right)$, we can choose $j_{\alpha} \in E_{\alpha}$ such that $\operatorname{cf}\left(j_{\alpha}\right)=\aleph_{1}$ and let $\delta_{\alpha}=\sup \left(w_{j_{\alpha}}^{\alpha}\right)$, now $w_{j_{\alpha}}^{\alpha}$ is closed under $\omega$-limits (as $\left\langle w_{j}^{\alpha}: j \leq \alpha\right\rangle$ is increasing continuous, $\left.j<\alpha \Rightarrow \operatorname{closure}\left(w_{j}^{\alpha}\right) \subseteq w_{j+1}^{\alpha}\right)$ and $\aleph_{1}=\operatorname{cf}\left(\operatorname{otp} w_{j_{\alpha}}^{\alpha}\right)$ so there is $\left\langle\beta_{\varepsilon}: \varepsilon<\omega_{1}\right\rangle$ increasing continuous with limit $\delta_{\alpha}, \beta_{\varepsilon} \in w_{j_{\alpha}}^{\alpha}$ so $\varepsilon<\zeta<$ $\omega_{1} \Rightarrow N_{j}^{\beta_{\varepsilon}}=N_{j}^{\beta_{\zeta}} \cap N_{\beta_{\varepsilon}}$ and easily $N_{j}^{\alpha} \cap N_{\beta_{\varepsilon}}=N_{j}^{\beta_{\varepsilon}}$ hence

$$
\begin{gathered}
\bigoplus M_{j_{\alpha}}^{\delta_{\alpha}}=\cap\left\{\bigcup_{j \in C} N_{j}^{\beta}: C \text { a club of } \delta_{\alpha}\right\} \\
\text { so } N_{j_{\alpha}}^{\alpha}=M_{j_{\alpha}}^{\delta_{\alpha}}(\text { see }[\text { Sh } 351, \S 4]) .
\end{gathered}
$$

By Fodor lemma for some $j^{*}, \alpha^{*}$ and stationary $S \subseteq S^{*}, \alpha \in S^{*} \Rightarrow j_{\alpha}=j^{*} \&$ $\delta_{\alpha}=\delta^{*}$. So for all $\alpha \in S, N_{j_{\alpha}}^{\alpha}$ are the same say $N^{*}$. So $N^{*} \in K_{\lambda}$, for $\alpha \in S$,

$q_{\alpha}=\operatorname{tp}\left(a_{\alpha}, N^{*}, N_{j_{\alpha}}^{\alpha+1}\right)$ extend $p_{\alpha}(\in \mathscr{S})$. Also if $r \in \mathscr{S}\left(N^{*}\right)$ is realized in $N_{j_{\alpha}}^{\alpha+1}$ say by $b$ (for some $\alpha \in S$ ) then no member of $\bigcup\left\{N_{j^{*}}^{\beta+1} \backslash N_{j^{*}}^{\beta}: \beta \in S \cap \alpha\right\}$ realizes it (holds by clause $(G)$, see clause $(F))$. So the sets $\Gamma_{\alpha}=\left\{\operatorname{tp}\left(b, N_{j^{*}}^{\delta^{*}}, N_{j^{*}}^{\alpha+1}\right): b \in N_{j^{*}}^{\alpha+1} \backslash N^{*}\right\}$ are pairwise disjoint and each has a member extending $p_{\alpha} \in \mathscr{S}$ (as exemplified by $a_{\alpha}$ and $p_{\alpha}$ is not extended by any $p \in \bigcup_{\beta<\alpha} \Gamma_{\beta}$ (as $p_{\alpha}$ is not realized in $\left.N_{\alpha}\right)$ ). $\square_{4.6}$

4.7 Claim. Assume $p^{*}$ is a counterexample to 4.4.

1) If $N \in K_{\lambda}, \Gamma \subseteq \mathscr{S}_{\geq p^{*}}(N),|\Gamma| \leq \lambda^{+} \underline{\text { then }}$ 


$$
\begin{array}{r}
\left\{p \in \mathscr{S}_{\geq p^{*}}(N): \text { for some } N^{\prime}, N \leq_{\mathfrak{K}} N^{\prime} \in K_{\lambda} \text { and some } b \in N^{\prime}\right. \\
\text { realizes } \left.p \text { but no } b \in N^{\prime} \text { realizes any } q \in \Gamma\right\}
\end{array}
$$

has cardinality $\geq \lambda^{++}$.

2) We can find $N \in K_{\lambda^{+}}$, and $N_{i}, N<_{\mathfrak{K}} N_{i} \in K_{\lambda^{+}}$for $i<2^{\lambda^{+}}$such that the set $\Gamma_{i}=\left\{\operatorname{tp}\left(a, N, N_{i}\right): a \in N_{i}\right\}$ are pairwise distinct, in fact, no one embeddable into another (so we get $I\left(\lambda^{+}, \mathfrak{K}\right)=2^{\lambda^{+}}$and if $\left(2^{\lambda}\right)^{+}<2^{\lambda^{+}}$then $\operatorname{IE}\left(\lambda^{+}, \mathfrak{K}\right)=2^{\lambda^{+}}$thus contradicting the categoricity in $\lambda^{+}$from the assumptions).

Proof. 1) Apply 4.6 with $N$ and $\mathscr{S}_{\geq p^{*}}(N)$ here standing for $N$ and $\mathscr{S}$ there, so we get $N^{*}, N_{i}\left(i<\lambda^{++}\right)$and $M^{*}$ such that $N^{*} \leq_{\mathfrak{K}} N_{i} \in \mathfrak{K}_{\lambda}$, and $\Gamma_{i}=\left\{\operatorname{tp}\left(a, N^{*}, N_{i}\right)\right.$ : $\left.a \in N_{i} \backslash N^{*}\right\}$ are pairwise disjoint and there are $p_{i} \in \Gamma_{i}, p_{i}\left\lceil N \in \mathscr{S}_{\geq p^{*}}(N)\right.$ pairwise distinct; now $p_{i}$ is not algebraic hence $p_{i} \in \mathscr{S}_{\geq p^{*}}\left(N^{*}\right)$. As $K$ is categorical in $\lambda$, without loss of generality $N^{*}=N$, so all but $\leq \lambda^{+}$of the models $N_{i}$ can serve as the required $N^{\prime}$.

2) Now by part (1) of 4.7 we can choose by induction on $i<\lambda^{+}$, $\left\langle\left(N_{\eta}, \Gamma_{\eta}\right): \eta \in{ }^{i} 2\right\rangle$ such that:

(a) $N_{\eta} \in K_{\lambda}$ and $\Gamma_{\eta} \subseteq \bigcup_{j<i} \mathscr{S}_{\geq p^{*}}\left(N_{\eta \uparrow j}\right)$ and $\left|\Gamma_{\eta}\right| \leq \lambda$

(b) if $\nu \triangleleft \eta$ then $N_{\nu} \leq_{\mathfrak{K}} N_{\eta}$ and $\Gamma_{\eta} \subseteq \Gamma_{\nu}$

(c) some $p \in \Gamma_{\eta^{\wedge}\langle 0\rangle}$ is from $\mathscr{S}\left(N_{\eta}\right)$ and is realized in $N_{\eta^{\wedge}\langle 1\rangle}$ (similarly for $\Gamma_{\eta^{\wedge}\langle 1\rangle}, N_{\eta^{\wedge}\langle 0\rangle}$ )

(d) if $i$ is a limit ordinal, then $N_{\eta}=\bigcup_{j<i} N_{\eta \uparrow j}$ and $\Gamma_{\eta}=\bigcup_{j<i} \Gamma_{\eta \uparrow j}$.

The successor case is done by 4.7(1) (you may object that the type in $\Gamma_{\eta}$ are not from $\mathscr{S}_{\geq p^{*}}\left(N_{\eta}\right)$ but from $\bigcup_{j<i} \mathscr{S}_{\geq p^{*}}\left(N_{\eta \uparrow j}\right)$ ? However they are minimal - see 4.5(1)). For $\eta \in{ }^{\lambda^{+}} 2$ let $N_{\eta}=\bigcup_{i<\lambda^{+}} N_{\eta \nmid i}$. Now by 1.4(1), $\left\{N_{\eta} / \cong: \eta \in{ }^{\lambda^{+}} 2\right\}$ has cardinality $2^{\lambda^{+}}$, contradiction.

4.8 Claim. 1) If $M_{0} \in K_{\lambda}, M_{1} \in K_{\lambda^{+}}$, and $M_{0} \leq_{\mathfrak{K}} M_{1}$ then every minimal $p \in \mathscr{S}\left(M_{0}\right)$ is realized in $M$.

2) Every $M_{1} \in K_{\lambda^{+}}$is saturated at least for minimal types (i.e. if $M_{0} \leq_{\mathfrak{K}} M_{1}$,

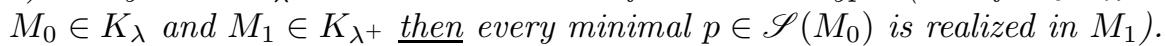

3) If $M \in K_{\lambda}$ then $\{p \in \overline{\mathscr{S}(M)}: p$ minimal $\}$ has cardinality $\leq \lambda^{+}$.

Proof. 1) Let $\bar{N}=\left\langle N_{\alpha}: \alpha<\lambda^{+}\right\rangle$be a representation of $N_{\lambda^{+}} \in K_{\lambda^{+}}$. Let $N \in K_{\lambda}, p \in \mathscr{S}(N)$ be minimal we ask

$(*)_{p}$ is there a club of $\alpha<\lambda^{+}$such that every $q \in \mathscr{S}_{\geq p}\left(N_{\alpha}\right)$ is realized in $\lambda^{+}$? 
By 4.4 there is $N_{\lambda^{+}}^{\prime} \in K_{\lambda^{+}}$for which the answer is yes, hence, as $\mathfrak{K}$ is categorical in $\lambda^{+}$, this holds for $N_{\lambda^{+}}$. So this holds for every minimal $p$. Now if $N^{\prime} \leq_{\mathfrak{K}} N_{\lambda^{+}}, N^{\prime} \in$ $K_{\lambda}$ and $p \in \mathscr{S}\left(N^{\prime}\right)$ is minimal then for some $\alpha, N^{\prime} \leq_{\mathfrak{K}} N_{\alpha}$ and for every $\beta \in\left[\alpha, \lambda^{+}\right)$, $p$ has a unique non-algebraic extension $p_{\beta} \in \mathscr{S}\left(N_{\beta}\right)$ (which necessarily is minimal). Now $p_{\beta} \in \mathscr{S}_{\geq p}\left(N_{\beta}\right)$ hence for a club of $\beta<\lambda^{+}, p_{\beta}$ is realized in $N_{\lambda^{+}}$, so we have finished the proof of part (1).

2) By part (1).

3) Follows by part (1).

From 4.3, 2.9(1) we can conclude

4.9 Conclusion. Every $\left(M_{0}, M_{1}, a\right) \in K_{\lambda}^{3}$ has the extension property. 


\section{$\S 5$ INEvitable TyPes AND STABILITy IN $\lambda$}

5.1 Hypothesis. Assume the model theoretic assumptions from 4.1 and

(d) there is a minimal member of $K_{\lambda}^{3}$ (follows from the conclusion of 3.28).

5.2 Definition. We call $p \in \mathscr{S}(N)$ inevitable if: $N \leq_{\mathfrak{K}} M \& N \neq M \Rightarrow$ some $c \in M$ realizes $p$.

We call $(M, N, a) \in K_{\lambda}^{3}$ inevitable if $\operatorname{tp}(a, M, N)$ is inevitable.

So by $4.3-4.8$ we shall deduce

5.3 Claim. 1) If there is a minimal triple in $K_{\lambda}^{3}$, then there is an inevitable $p=\operatorname{tp}\left(a, N, N_{1}\right)$ with $\left(N, N_{1}, a\right) \in K_{\lambda}^{3}$ minimal.

2) Moreover, if $p_{0} \in \mathscr{S}\left(N_{0}\right)$ is minimal, $N_{0} \in K_{\lambda}$ then we can find $N_{1}, N_{0} \leq_{\mathfrak{K}} N_{1} \in$ $K_{\lambda}$ such that the unique non-algebraic extension $p_{1}$ of $p_{0}$ in $\mathscr{S}\left(N_{1}\right)$ is inevitable.

Proof of 5.3. 1) Follows by part (2).

2) Let $\left(M_{0}, M_{1}, a\right) \in K_{\lambda}^{3}$ be minimal and $p_{0}=\operatorname{tp}\left(a, M_{0}, M_{1}\right)$. We try to choose by induction on $i$ a model $N_{i}$ such that: $N_{0}=M_{0}, N_{i} \in K_{\lambda}$ is $\leq_{\mathfrak{K}^{-}}$-increasing continuously and $N_{i}$ omits $p_{0}, N_{i} \neq N_{i+1}$. If we succeed, $\bigcup_{i<\lambda^{+}} N_{i}$ is a member of $K_{\lambda^{+}}$which is non-saturated for minimal types, contradicting 4.8(2). As for $i=0$, $i$ limit we can define, necessarily for some $i$ we have $N_{i}$ but not $N_{i+1}$. Now $p_{0}$ has a unique extension in $\mathscr{S}\left(N_{i}\right)$ which we call $p_{i}$ and $p_{0}$ has no algebraic extension in $\mathscr{S}\left(N_{i}\right)$.

[why? as $N_{i}$ omits $p_{0}$ ]. So $p_{i}$ is the unique extension of $p_{0}$ in $\mathscr{S}\left(N_{i}\right)$ [by 4.5(1)], and so

(*) if $N_{i} \leq_{\mathfrak{K}} N^{\prime} \in K_{\lambda}$ and $N^{\prime} \neq N$, then $p_{i}$ is realized in $N^{\prime}$.

By L.S. we can omit " $N^{\prime} \in K_{\lambda}$ ", so $\left(N_{i}, p_{i}\right)$ are as required.

5.4 Fact. Inevitable types have few $(\leq \lambda)$ conjugates (i.e. for $p \in \mathscr{S}\left(M_{0}\right)$ inevitable $M_{0} \in K_{\lambda}, M_{1} \in K_{\lambda}$ we have $\left.\left|\mathscr{S}_{p}\left(M_{1}\right)\right| \leq \lambda\right)$, moreover $\mid\{p \in \mathscr{S}(N)$ : $p$ inevitable $\} \mid \leq \lambda$ for $N \in K_{\lambda}$.

Proof. Easy.

The following construction plays a central role in what remains of this paper.

5.5 Claim. For any limit $\alpha<\lambda^{+}$, we can find $\left\langle N_{i}: i \leq \alpha\right\rangle$ and $\left\langle p_{i}: i \leq \alpha\right\rangle$ such that:

(i) $N_{i} \in K_{\lambda}$,

(ii) $N_{i}$ is $\leq_{\mathfrak{K}}$-increasing continuous

(iii) $p_{i} \in \mathscr{S}\left(N_{i}\right)$ is minimal, 
(iv) $p_{i}$ increases continuously (see 4.5(3))

(v) $p_{0}$ is inevitable

(vi) $p_{\alpha}$ is inevitable

(vii) $N_{i} \neq N_{i+1}$ moreover some $c \in N_{i+1} \backslash N_{i}$ realizes $p_{0}$ (hence $p_{i}$ ).

Remark. Why not just build a non-saturated model in order to prove 5.5? Works, too.

Proof. Choose $N^{0}<_{\mathfrak{K}} N^{1}$ in $K_{\lambda^{+}}$(so $N^{0} \neq N^{1}$ ), such a pair exists as $K_{\lambda^{+2}} \neq \emptyset$. Let $N^{\ell}=\bigcup_{i<\lambda^{+}} N_{i}^{\ell}$ with $N_{i}^{\ell} \in K_{\lambda}$ being $\leq_{\mathfrak{K}^{-}}$-increasing continuously in $i$. Now $E_{0}=\left\{\delta<\lambda^{+}: N_{\delta}^{1} \neq N_{\delta}^{0}\right.$ and $\left.N_{\delta}^{1} \cap N^{0}=N_{\delta}^{0}\right\}$ is a club of $\lambda^{+}$. Without loss of generality $E_{0}=\lambda^{+}$.

For each $c \in N^{1} \backslash N^{0}$, the set

$$
X_{c}=:\left\{i<\lambda^{+}: c \in N_{i}^{1} \text { and }\left(N_{i}^{0}, N_{i}^{1}, c\right) \text { is minimal }\right\}
$$

is empty or an end segment of $\lambda^{+}$hence

$$
\begin{aligned}
& E_{1}=\left\{\delta<\lambda^{+} \text {:(i) } \delta\right. \text { limit } \\
& \text { (ii) } \text { if } i<\delta \text { and } p \in \mathscr{S}\left(N_{i}^{0}\right) \text { is minimal inevitable } \\
& \text { and realized in } N^{0} \backslash N_{\delta}^{0} \text { then it is } \\
& \text { realized in } N_{\delta}^{0} \backslash N_{i}^{0} \text { (actually automatic) } \\
& \text { (iii) if } c \in N_{\delta}^{1} \backslash N^{0} \text { (hence } \exists i<\delta, c \in N_{i}^{1} \text { ) and } X_{c} \\
& \text { is non-empty then } \left.\delta \in X_{c} \text { and } \min \left(X_{c}\right)<\delta\right\}
\end{aligned}
$$

is a club of $\lambda^{+}$(see 5.4).

Now for $\delta \in E_{1}$, we have $N_{\delta}^{0}<_{\mathfrak{K}} N_{\delta}^{1}$, so by 5.3(1) there is $c_{\delta} \in N_{\delta}^{1} \backslash N_{\delta}^{0}$ such that:

$$
\begin{gathered}
\left(N_{\delta}^{0}, N_{\delta}^{1}, c_{\delta}\right) \text { is minimal } \\
\operatorname{tp}\left(c_{\delta}, N_{\delta}^{0}, N_{\delta}^{1}\right) \text { is inevitable }
\end{gathered}
$$

As $\delta$ is limit, for some $i<\delta, c \in N_{i}^{1}$, also $\delta \in X_{c}$ hence there is $j$ such that: $i<j<\delta \quad \& \quad j \in X_{c}$ hence $\left(N_{j}^{0}, N_{j}^{1}, c\right)$ is minimal; choose such $j_{\delta}, c_{\delta}$. Let $\kappa=\operatorname{cf}(\kappa)=\operatorname{cf}(\alpha) \leq \lambda$, so for some $j^{*}, c^{*}$ we have

$$
S=\left\{\delta \in E_{1}: \operatorname{cf}(\delta)=\kappa, j_{\delta}=j^{*}, c_{\delta}=c^{*}\right\}
$$

is stationary in $\lambda^{+}$. 
Choose $e$ closed $\subseteq E_{1}$ of order type $\alpha+1$ with first element and last element in $S$; for $\zeta \in\left[j^{*}, \lambda^{+}\right)$let $p_{\zeta}=\operatorname{tp}\left(c^{*}, N_{\zeta}^{0}, N_{\zeta}^{1}\right)$. (In fact, we could have: all nonaccumulation member of $e$ are in $S$; no real help.)

Now $\left\langle N_{\zeta}^{0}, p_{\zeta}: \zeta \in e\right\rangle$ is as required (up to re-indexing)(clause (viii) holds by clause (ii) in the definition of $E_{1}$ ).

5.6 Claim. Assume $\left\langle N_{i}, p_{i}: i \leq \alpha\right\rangle$ is as in 5.5, $\alpha<\lambda$ divisible by $\lambda$. Then any $p \in \mathscr{S}\left(N_{0}\right)$ is realized in $N_{\alpha}$, moreover $N_{\alpha}$ is universal in $K_{\lambda}$ over $N_{0}$.

Proof. (Similar to the proof of 0.25 ; which is [Sh 300, II,§3]).

Let $N_{0} \leq_{\mathfrak{K}} M_{0} \in K_{\lambda}, a \in M_{0} \backslash N_{0}$ we shall show that $\operatorname{tp}\left(a, N_{0}, M_{0}\right)$ is realized in $N_{\alpha}$.

Let $\alpha=\bigcup_{i<\lambda} S_{i},\left\langle S_{i}: i<\lambda\right\rangle$ pairwise disjoint, each $S_{i}$ unbounded in $\alpha, \lambda$ divides $\operatorname{otp}\left(S_{i}\right)$ and $\operatorname{Min}\left(S_{i}\right) \geq i$. We choose by induction on $i \leq \alpha$ the following:

$$
N_{i}^{1}, M_{i}^{1}, h_{i},\left\langle a_{\zeta}: \zeta \in S_{i}\right\rangle \quad \text { (the last one only if } i<\alpha \text { ) }
$$

such that:

(a) $N_{i}^{1} \leq_{\mathfrak{K}} M_{i}^{1}$ are in $K_{\lambda}$

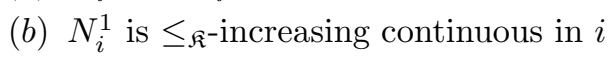

(c) $M_{i}^{1}$ is $\leq_{\mathfrak{K}^{-}}$-increasing continuous in $i$

(d) $\left(N_{0}^{1}, M_{0}^{1}\right)=\left(N_{0}, M_{0}\right)$

(e) $\left\langle a_{\zeta}: \zeta \in S_{i}\right\rangle$ is a list of $\left\{c \in M_{i}^{1}: c\right.$ realizes $\left.p_{0}\right\}$

(f) $h_{i}$ is an isomorphism from $N_{i}$ onto $N_{i}^{1}$

(g) $j<i \Rightarrow h_{j} \subseteq h_{i}$ and $h_{0}=\operatorname{id}_{N_{0}}$

(h) $a_{i} \in N_{i+1}^{1}$ (note: $M_{i}^{1} \cap N_{i+1}^{1} \neq N_{i}^{1}$ in general).

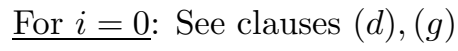

$$
N_{0}^{1}=N_{0}, M_{0}^{1}=M_{0}, h_{0}=\operatorname{id}_{N_{0}} .
$$

For $i=$ limit: Let $N_{i}^{1}=\bigcup_{j<i} N_{j}^{1}$ and $M_{i}^{1}=\bigcup_{j<i} M_{j}^{1}$ and $h_{i}=\bigcup_{j<i} h_{j}$ and lastly choose $\left\langle a_{\zeta}: \zeta \in S_{i}\right\rangle$ by clause $(e)$.

For $i=j+1$ : Note $a_{j}$ is already defined, it belongs to $M_{j}^{1}$ and it realizes $p_{0}$.

Case 1: $a_{j} \in N_{j}^{1}$ (so clause $(h)$ is no problem).

Use amalgamation on $N_{j}, N_{i}, M_{j}^{1}$ and the mapping $\operatorname{id}_{N_{j}}$, $h_{i}$,i.e.

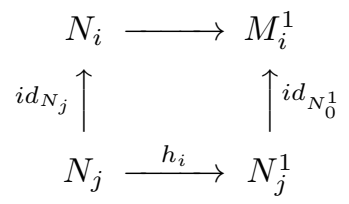


Case 2: $a_{j} \notin N_{j}^{1}$.

Then $\operatorname{tp}\left(a_{j}, N_{j}^{1}, M_{j}^{1}\right)$ is not algebraic, extending the minimal type $p_{0} \in \mathscr{S}\left(N_{0}\right)$. Also by clause (viii) of 5.5 there is $c \in N_{i} \backslash N_{j}$ which realizes $p_{0}$. As $p_{0} \in \mathscr{S}(N)$ is minimal

$$
h_{j}\left(\operatorname{tp}\left(c, N_{j}, N_{i}\right)\right)=\operatorname{tp}\left(a_{j}, N_{j}^{1}, M_{j}^{1}\right)
$$

so acting as in Case 1 we can also guarantee $h_{i}(c)=a_{j}$, so $a_{j} \in \operatorname{Rang}\left(h_{i}\right)=N_{i}^{1}$ as required.

In the end we have $N_{\alpha}^{1} \leq_{\mathfrak{K}} M_{\alpha}^{1}$. If $N_{\alpha}^{1}=M_{\alpha}^{1}$, then $h_{\alpha}^{-1} \uparrow M_{0}=h_{\alpha}^{-1} \uparrow M_{0}^{1}=$ $h_{\alpha}^{-1} \uparrow N_{0}^{1}$ show that $M_{0}$ can be embedded into $N_{\alpha}$ over $N_{0}$ as required. So assume $N_{\alpha}^{1}<_{\mathfrak{K}} M_{\alpha}^{1}$. Now $p_{\alpha} \in \mathscr{S}\left(N_{\alpha}\right)$ is inevitable hence $h_{\alpha}\left(p_{\alpha}\right) \in \mathscr{S}\left(N_{\alpha}^{1}\right)$ is inevitable. Hence some $d \in M_{\alpha}^{1} \backslash N_{\alpha}^{1}$ realizes $h_{\alpha}\left(p_{\alpha}\right)$ hence $d$ realizes $h_{\alpha}\left(p_{\alpha}\right)\left\lceil N_{0}^{1}=p_{0}\right.$; also $\alpha$ is a limit ordinal so for some $i<\alpha, d \in M_{i}^{1}$ hence for some $\zeta \in S_{i}$ we have $a_{\zeta}=d$, hence

$$
d=a_{\zeta} \in N_{\zeta+1}^{1} \subseteq N_{\alpha}^{1}
$$

contradicting the choice of $d$.

So we are done.

5.7 Conclusion. If $N \in K_{\lambda}$ then:

(a) $|\mathscr{S}(N)|=\lambda$

(b) there is $N_{1}, N<_{\mathfrak{K}} N_{1} \in K_{\lambda}$ such that $N_{1}$ is universal over $N$ in $K_{\lambda}$

(c) for any regular $\kappa \leq \lambda$ we can demand that $\left(N_{1}, c\right)_{c \in N}$ is $(\lambda, \kappa)$-saturated (see $0.6(1)$ )

5.8 Remark. In fact amalgamation in $\lambda$ and stability in $\lambda$ (i.e. (a) of 5.7) implies (b) and $(c)$ of 5.7 .

5.9 Conclusion. The $N \in K_{\lambda^{+}}$is saturated above $\lambda$ (i.e. over models in $K_{\lambda}$ !).

5.10 Claim. Assume $\kappa=c f(\kappa) \leq \lambda$.

There are $N_{0}, N_{1}, a, N_{0}^{+}, N_{1}^{+}$such that

(i) $\left(N_{0}, N_{1}, a\right) \in K_{\lambda}^{3}$ and

(ii) $\left(N_{0}, N_{1}, a\right) \leq\left(N_{0}^{+}, N_{1}^{+}, a\right) \in K_{\lambda}^{3}$ and

(iii) $\left(N_{0}^{+}, c\right)_{c \in N_{0}}$ is $(\lambda, \kappa)$-saturated,

(iv) tp $\left(a, N_{0}, N_{1}\right)$ is minimal inevitable and

(v) $\operatorname{tp}\left(a, N_{0}^{+}, N_{1}^{+}\right)$is minimal inevitable. 
Proof. As in the proof of 5.5 as

$$
\begin{gathered}
E_{2}=\left\{\delta: \text { for every } i<\delta,\left(N_{\delta}^{0}, c\right)_{c \in N_{i}}\right. \text { is saturated } \\
\text { of cofinality } \operatorname{cf}(\delta)\}
\end{gathered}
$$

is a club of $\lambda^{+}$.

5.11 Claim. 1) In $K_{\lambda}$ we have disjoint amalgamations.

2) If $M \leq_{\mathfrak{K}} N$ are in $K_{\lambda}$ and $p \in \mathscr{S}(M)$ non-algebraic then for some $N^{\prime}$, c we have: $N \leq_{\mathfrak{K}} N^{\prime} \in K_{\lambda}$ and $c \in N^{\prime} \backslash N$ realizes $p$.

Proof. 1) First note

$\otimes$ if $M \leq_{\mathfrak{K}} N$ in $K_{\lambda}$ we can find $\alpha<\lambda^{+}$, and sequence $\left\langle M_{i}: i \leq \alpha\right\rangle$ which

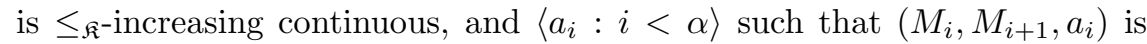
minimal and reduced and $N \leq_{\mathfrak{K}} M_{\alpha}, M=M_{0}$.

[Why? There is a minimal reduced pair, hence we can find $\left\langle M_{i}: i<\right.$ $\left.\lambda^{+}\right\rangle \leq_{\mathfrak{K}^{-}}$-increasing continuous, $\left(M_{i}, M_{i+1}, a_{i}\right)$ minimal reduced and $M=$ $M_{0}$. So by 5.9 we know $\bigcup_{i<\lambda^{+}} M_{i} \in K_{\lambda^{+}}$is saturated, hence we can embed $N$ into $\bigcup_{i<\lambda^{+}} M_{i}$ over $N$ so this embedding is into some $M_{\alpha}, \alpha<\lambda^{+}$.]

So given $M \leq_{\mathfrak{K}} M^{1}, M^{2}$, without loss of generality $M^{\ell}=M_{\alpha_{\ell}}^{\ell},\left\langle\left(M_{i}^{\ell}, a_{i}^{\ell}\right): i \leq \alpha_{\ell}\right\rangle$ as above, and start to amalgamate using the extension property and "reduced".

5.12 Remark. We could prove 5.11 earlier using "reduced triples". I.e. note that for some $\left\langle M_{i}^{1}: i<\lambda^{+}\right\rangle \in \mathbf{S} e q_{\lambda^{+}}\left[\mathbf{C}_{\mathfrak{K}, \lambda^{+}}^{1}\right]$, for each $i$ for some $a$ the triple $\left(M_{i}^{1}, M_{i+1}^{1}, a\right) \in$ $K_{\lambda}^{3}$ is reduced. Hence if $M \leq_{\mathfrak{K}} N$ from $K_{\lambda}$, for some $\bar{M}=\left\langle M_{i}: i \leq \alpha\right\rangle \leq_{\mathfrak{K}^{-}}$ increasing continuous, $\left\langle M_{i}, M_{i+1}, b_{i}\right) \in K_{\lambda}^{3}$ is reduced, $M_{0}=M, N \leq M_{\alpha} \in K_{\lambda}$ (otherwise find $\left\langle M_{i}^{2}: i<\lambda^{+}\right\rangle \in \mathbf{S} e q_{\lambda^{+}}\left[\mathbf{C}_{\mathfrak{K}^{1} \lambda^{+}}^{1}\right]$ with $\left(M_{i}, M_{i+1}\right) \cong(M, N)$, hence $M^{1}=\bigcup_{i<\lambda^{+}} M_{i}^{1}, M^{2}=\bigcup_{i<\lambda^{+}} M_{i}^{2}$ are non-isomorphic members of $K_{\lambda^{+}}$, contradiction). Now prove by induction on $\beta \leq \alpha$ that if $M \leq_{\mathfrak{K}} N_{0} \in K_{\lambda}$ then $N_{0}, M_{\beta}$ has disjoint amalgamation over $M_{0}=M$ (i.e. we need to decompose only one side).

5.13 Question: If $M \in K_{\lambda}, p \in \mathscr{S}(M)$ is minimal, is it reduced? Or at least, if $M_{0} \leq_{\mathfrak{K}} M_{1}$ are in $K_{\lambda}, p_{1} \in \mathscr{S}\left(M_{\ell}\right)$, no algebraic $p_{0}=p_{1} \uparrow M_{0}, p_{0}$ is minimal and reduced is also $p_{1}$ reduced?

Probably true, but not needed for our aims (here and in [Sh 600], 7.6(2)) is an approximation. Can prove it if $\lambda<\lambda^{\aleph_{0}}$ or there are E.M. models. 
$\S 6$ Proving For $\mathfrak{K}$ CATEgorical IN $\lambda^{+2}$

6.1 Hypothesis. Assume the model theoretic assumptions from $4.1+5.1$ and so the further model theoretic properties deduced in $\S 4+\S 5$.

We use heavily 4.8 .

6.2 Definition. 1) We say $\left(M_{0}, M_{1}, M_{2}\right) \in K_{\lambda}^{\text {uniq }}$ (has unique amalgamation in $K_{\lambda}$ ) when

(a) $M_{0}, M_{1}, M_{2} \in K_{\lambda}$

(b) $M_{0} \leq_{\mathfrak{K}} M_{1}$ and $M_{0} \leq_{\mathfrak{K}} M_{2}$

(c) if for $i=1,2$ we have $g_{\ell}^{i}: M_{\ell} \rightarrow N_{i} \in K_{\lambda}$ such that:

(i) $g_{\ell}^{i}$ a $\leq_{\mathfrak{K}}$-embedding,

(ii) $g_{0}^{i} \subseteq g_{1}^{i}$ and $g_{0}^{i} \subseteq g_{2}^{i}$ for $i=1,2$

(iii) $\operatorname{Rang}\left(g_{1}^{i}\right) \cap \operatorname{Rang}\left(g_{2}^{i}\right)=\operatorname{Rang}\left(g_{0}^{i}\right)$

(disjoint amalgamation) for $i=1,2$

then we can find $N \in K_{\lambda}$ and $\leq_{\mathfrak{R}^{-}}$-embeddings

$$
f^{i}: N_{i} \rightarrow N \text { for } i=1,2
$$

such that

$$
\bigwedge_{\ell<3} f^{1} \circ g_{\ell}^{1}=f^{2} \circ g_{\ell}^{2}
$$

2) Let $K_{\lambda}^{2 \text {,uq }}$ is the class of pairs $\left(M_{0}, M_{2}\right)$ such that $M_{0} \leq_{\mathfrak{K}} M_{2}$ are both in $K_{\lambda}$ and $\left[M_{0} \leq_{\mathfrak{K}} M_{1} \in K_{\lambda} \Rightarrow\left(M_{0}, M_{1}, M_{2}\right) \in K_{\lambda}^{\text {uniq }}\right]$ and let $K_{\lambda}^{3, \text { uq }}$ be the class of pairs $\left(M_{0}, M_{2}\right) \in K_{\lambda}^{2, \text { uq }}$ satisfying $M_{0} \neq M_{2}$.

6.3 Claim. 1) If $\left(M_{0}, M_{1}, M_{2}\right) \in K_{\lambda}^{\text {uniq }} \underline{\text { then }}$

(a) $\left(M_{0}, M_{2}, M_{1}\right) \in K_{\lambda}^{\text {uniq }}$

(b) if $M_{0} \leq_{\mathfrak{K}} M_{2}^{\prime} \leq_{\mathfrak{K}} M_{2} \underline{\text { then }}\left(M_{0}, M_{1}, M_{2}^{\prime}\right) \in K_{\lambda}^{\text {uniq }}$.

2) Assume $M_{0} \leq_{\mathfrak{K}} M_{2}$ are from $K_{\lambda}$ and $M_{1} \in K_{\lambda}$ is universal over $M_{0}$. Then $\left(M_{0}, M_{2}\right) \in K_{\lambda}^{2 \text {,uq }} \Leftrightarrow\left(M_{0}, M_{1}, M_{2}\right) \in K_{\lambda}^{\text {uniq }}$.

Proof. 1)a) Trivial.

b) Chase arrows (using disjoint amalgamation; i.e. 5.11).

2) Follows by $6.3(1)(\mathrm{a})+(\mathrm{b})$ and the definition. 
6.4 Lemma. Suppose

$\otimes$ there is $\left(M_{0}, M_{1}, M_{2}\right) \in K_{\lambda}^{\text {uniq }}$ such that $M_{0} \neq M_{2}$ and $M_{1}$ is universal over $M_{0}$.

Then: there are $N^{0}<_{\mathfrak{K}} N^{1}$ in $K_{\lambda^{+}}$such that:

(a) $N^{0} \neq N^{1}$

(b) for every $c \in N^{1} \backslash N^{0}$ there is $M=M_{c}$ satisfying $N^{0} \leq_{\mathfrak{K}} M \leq_{\mathfrak{K}} N^{1}$ and $N^{0} \neq M$ and $c \in N^{1} \backslash M$.

Proof. Choose $\left\langle N_{i}^{0}: i<\lambda^{+}\right\rangle$, a sequence of members of $K_{\lambda}$ which is $\leq_{\mathfrak{K}^{-}}$-increasing continuous, such that:

$$
\left(N_{i}^{0}, N_{i+1}^{0}\right) \cong\left(M_{0}, M_{2}\right)
$$

So $N_{i}^{0} \neq N_{i+1}^{0}$ hence $N_{0}=\bigcup_{i<\lambda^{+}} N_{i}^{0} \in K_{\lambda^{+}}$and without loss of generality $\left|N_{0}\right|=\lambda^{+}$.

We now choose by induction $i<\lambda^{+}, N_{i}^{1}$ and $M_{i, c}$ for $c \in N_{i}^{1} \backslash N_{i}^{0}$ such that:

(a) $N_{i}^{0} \leq_{\mathfrak{K}} N_{i}^{1} \in K_{\lambda}$ and $N_{i}^{0} \neq N_{i}^{1}$

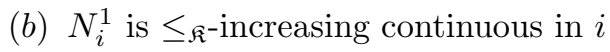

(c) $j<i \Rightarrow N_{j}^{1} \cap N_{i}^{0}=N_{j}^{0}$; moreover $N_{i}^{1} \cap\left|N_{0}\right|=N_{i}^{0}$

(d) $N_{i}^{0} \leq_{\mathfrak{K}} M_{i, c} \leq_{\mathfrak{K}} N_{i}^{1}$

(e) $c \notin M_{i, c}$

(f) $N_{i}^{0} \neq M_{i, c}$

(g) if $j<i$ and $c \in N_{j}^{1} \backslash N_{j}^{0}$ then $M_{i, c} \cap N_{j}^{1}=M_{j, c}$.

For $i=0$ : Choose $N_{i}^{1}$ such that

$N_{i}^{0} \leq_{\mathfrak{K}} N_{i}^{1},\left(N_{i}^{1}, c\right)_{c \in N_{i}^{0}}$ saturated (any cofinality will do) then by disjoint amalgamation easy to define the $M_{0, c}$ (remembering clause (c)).

For $i$ limit: Straightforward.

For $i=j+1$ : First we disjointly amalgamate getting $N_{i}^{\prime} \in K_{\lambda}$ such that $N_{i}^{0} \leq N_{i}^{\prime}, N_{j}^{1} \leq_{\mathfrak{K}} N_{i}^{\prime}$ and $\left|N_{i}^{\prime}\right| \cap\left|N_{0}\right|=\left|N_{i}^{0}\right|$ (as set of elements).

Let $N_{i}^{1}$ be such that:

$$
\begin{gathered}
N_{i}^{\prime} \leq_{\mathfrak{K}} N_{i}^{1} \in K_{\lambda} \\
\left(N_{i}^{1}, c\right)_{c \in N_{i}^{\prime}} \text { is saturated (any cofinality will do) } \\
\left|N_{i}^{1}\right| \cap\left|N_{0}\right|=\left|N_{i}^{0}\right| .
\end{gathered}
$$

Lastly we shall find the $M_{i, c}$ 's, the point is that $\left(N_{j}^{0}, N_{i}^{0}, N_{j}^{1}\right) \in K_{\lambda}^{\text {uniq }}$ (by $6.3(2))$. 
By $\otimes$ and Claim 6.3 we could have done the amalgamation in two steps and use uniqueness. Then by uniqueness of saturated extensions embed the result inside $N_{i}^{1}$ and similarly deal with new $c$ 's.

Now let $N_{1}=: \bigcup_{1<\lambda^{+}} N_{i}^{1}$ and for $c \in N_{1} \backslash N_{0}$ let $M_{c}=\bigcup\left\{M_{i, c}: c \in N_{i}^{\prime}\right\}$ they are as required.

Remark. The proof of 6.5 below is like [Sh 88 , proof of 3.8 stage(c)]. The aim is to contradict that under $I\left(\lambda^{+}, \mathfrak{K}\right)=0$ there are maximal triples.

6.5 Conclusion. Assume $\mathfrak{K}$ has amalgamation in $\lambda^{+}$. If $\otimes$ of 6.4 , then there is no maximal triple $(M, N, a)$ in $K_{\lambda^{+}}^{3}$.

Proof. We can get by 6.4 a contradiction.

[Why? Assume $\left(N_{0}, N_{2}, a\right) \in K_{\lambda}^{3}$ maximal, $\left(N^{0}, N^{1}\right)$ as in the conclusion (i.e. (a) $+\left(\right.$ b) ) of 6.4 ; by categoricity in $\lambda^{+}$without loss of generality $N_{0}=N^{0}$ and let $N_{1}=N^{1}$. Now $\mathfrak{K}$ has amalgamation for $\lambda^{+}$so there are $N \in K_{\lambda^{+}}$and $f$ such that $f: N_{2} \rightarrow N$ is a $\leq_{\mathfrak{K}}$-embedding of $N_{2}$ into $N$ over $N_{0}$ and $N_{1} \leq_{\mathfrak{K}} N$. If $f(a) \notin N_{1}$, then $\left(N_{0}, N_{2}, a\right)<_{f}\left(N_{1}, N, f(a)\right)$ contradict maximality. If $f(a) \in N_{1}$, then $M_{f(a)}$ is well defined (see 6.4) and $\left(N_{0}, N_{2}, a\right)<_{f}\left(M_{f(a)}, N, f(a)\right)$ contradicts maximality.]

6.6 Remark. 1) Another proof is to replace the assumption " $\mathfrak{K}$ has amalgamation in $\lambda^{+}$" by $I\left(\lambda^{+}, K\right)<2^{\lambda^{+2}}$. We start with $N_{0}, N_{1}, N_{2}, a$ as above and building, for every $S \subseteq \lambda^{+2}$, a sequence $\left\langle M_{\alpha}^{S}: \alpha<\lambda^{+2}\right\rangle$ of members of $K_{\lambda^{+}}$, which is $\leq_{\mathfrak{K}}$-increasing continuous, and $\alpha \in S \Rightarrow\left(M_{\alpha}^{S}, M_{\alpha+1}^{S}, a_{\alpha}^{S}\right) \cong\left(N_{0}, N_{2}, a\right)$, and $\alpha \in$ $\lambda^{+2} \backslash S \Rightarrow\left(M_{\alpha}^{S}, M_{\alpha+1}^{S}\right) \cong\left(N_{0}, N_{1}\right)$ which are as in (a) + (b) of 6.4. Let $M^{S}=$ $\bigcup_{\alpha<\lambda^{+2}} M_{\alpha}^{S} \in K_{\lambda^{+2}}$ and from $M^{S} / \cong$ we can reconstruct $S / \mathscr{D}_{\lambda^{+2}}$. So here we use $I\left(\lambda^{+2}, K\right)<2^{\lambda^{+2}}$ but not the definitional weak diamond for $\lambda^{++}$.

2) Note that if $2^{\lambda^{+}}<2^{\lambda^{+}}$then the assumption of 6.6(1) implies the assumption of 6.5 .

6.7 Claim. Assume

(*) $2^{\lambda}<2^{\lambda^{+}}<2^{\lambda^{++}}$

(or at least the definitional weak diamond for $\lambda^{+}, \lambda^{++}$).

If $\otimes$ of 6.4 fails, we get $I\left(\lambda^{+2}, K\right) \geq \mu_{\mathrm{wd}}(\lambda)$.

Proof. By 3.23 and 6.8 below.

The following serves to prove 6.7 
6.8 Claim. Assume $M \in K_{\lambda} \Rightarrow|\mathscr{S}(M)| \leq \lambda$.

If $K_{\lambda}^{3, \mathrm{uq}}=\emptyset$ (see Definition 6.2(2)), then there is $F$, an amalgamation choice function $F$ for $\mathbf{C}=\mathbf{C}_{\mathfrak{K}, \lambda^{+}}^{0}$ with the weak $\lambda^{+}$-coding property.

Proof. The point is that if $\bar{M}=\left\langle M_{\alpha}: \alpha<\lambda^{+}\right\rangle \in \mathbf{S} e q_{\lambda^{+}}[\mathbf{C}]$ and $\alpha<\lambda^{+}, M_{\alpha}<_{\mathfrak{K}}$ $N \in K_{\lambda}$, then for some $\beta \in\left(\alpha, \lambda^{+}\right)$we have:

$M_{\beta}$ is universal over $M_{\alpha}$, so as $K_{\lambda}^{3 \text {,uq }}=\emptyset$ necessarily $\left(M_{\alpha}, M_{\beta}, N\right) \notin K_{\lambda}^{\text {uniq }}$ and rest should be clear.

Of course, we use the extension property.

6.9 Remark. We can work in the context of $\S 3$, we need the existence of a saturated (equivalent by super limit) $M \in K_{\lambda^{+}}$. We now say how to replace $\mu_{\mathrm{wd}}\left(\lambda^{+2}\right)$ by $2^{\lambda^{+2}}$.

6.10 Claim. 1) Assume each $M \in K_{\lambda^{+}}$is saturated above $\lambda$.

If $(M, N, a) \in K_{\lambda}^{3}$ it and every $\left(M^{\prime}, N^{\prime}, a\right) \in K_{\lambda}^{3}$ above it has the extension property but for every $\left(M^{\prime \prime}, N^{\prime \prime}, a\right) \geq(N, N, a)$ (all in $K_{\lambda^{+}}^{3}$ for some $M^{*} \geq_{\mathfrak{K}} M^{\prime \prime}$ from $K_{\lambda^{+}}$, in amalgamation $\left(M^{*}, N^{*}, a\right) \geq\left(M^{\prime \prime}, N^{\prime \prime}\right.$, a) the type of $M^{*} \cup N$ inside $N^{*}$ is not determined then some $F$ (actually $\mathbf{F}^{*}$ ) has the $\lambda^{+}$-coding.

2) If above we just require that the type of $M^{*} \cup N^{\prime \prime}$ inside $N^{*}$ is not determined, then some $F$ (actually $\mathbf{F}^{*}$ ) has weak $\lambda^{+}$-coding.

3) We can restrict ourselves to disjoint embedding; i.e. use $\left(K_{\lambda^{+}}^{3}, \leq_{\mathrm{dj}}\right)$.

6.11 Discussion. 1) We get $\operatorname{IE}\left(\lambda^{+2}, \mathfrak{K}\right)=2^{\lambda^{+2}}$ when $\left(2^{\lambda^{+}}\right)^{+}<2^{\lambda^{+2}}$. See more in [Sh 600].

6.12 Theorem. Assume $I\left(\lambda^{+2}, K\right)<\mu_{\mathrm{wd}}\left(\lambda^{+2}\right)$ and $(*)$ of 6.7 (or at least the conclusion of 6.7). Then $I\left(\lambda^{+2}, K\right)=1 \Rightarrow I\left(\lambda^{+3}, K\right)>0$.

Remark. As in [Sh 88, §3].

Proof. By 0.20(1) it is enough to show that for some $M \in K_{\lambda^{++}}$there is $M^{\prime}$, $M \leq_{\mathfrak{K}} M^{\prime} \in K_{\lambda^{++}}, M \neq M^{\prime}$.

[Why? As then we can choose by induction on $i<\lambda^{+3}$ models $M_{i} \in K_{\lambda^{+2}}$, $\leq_{\mathfrak{K}^{-}}$-increasing continuous, $M_{i} \neq M_{i+1}$, for $i=0$ use $K_{\lambda^{+2}} \neq \emptyset$, for $i$ limit take union, for $i=j+1$ use the previous sentence; so $M_{\lambda^{+3}}=\cup\left\{M_{i}: i<\lambda^{+3}\right\} \in K_{\lambda^{+3}}$ as required.]

By 6.7 , the statement $\bigotimes$ of 6.4 holds so we can find $\left(N^{0}, N^{1}\right)$ as there so by 6.5 there is in $K_{\lambda^{+}}^{3}$ no maximal member. This implies (easy, see 2.6(6)) that there are $M^{*} \leq_{\mathfrak{K}} N^{*}$ from $K_{\lambda^{+2}}$ such that $M^{*} \neq N^{*}$ which as said above (by categoricity in $\left.\lambda^{+}\right)$, suffices. 


\section{$\S 7$ Extensions ANd CONJUGaCY}

7.1 Hypothesis. Assume the model theoretic assumptions from $4.1+5.2$ and the further model theoretic properties deduced since then (but not in 6.7,6.12) or just

(a) $\mathfrak{K}$ abstract elementary class

(b) $\mathfrak{K}$ has amalgamation in $\lambda$

(c) $\mathfrak{K}$ is categorical in $\lambda$ (can be weakened)

$(d) \mathfrak{K}$ is stable in $\lambda$ (see 5.7 , clause $(a)$ )

(e) there is an inevitable $p \in \mathscr{S}(N)$ for $N \in K_{\lambda}$ (holds by 5.3)

$(f)$ the basic properties in type theory.

We now continue toward eliminating the use of $I\left(\lambda^{++}, K\right)=1$ (in 6.12), and give more information. We first deal with the nice types in $\mathscr{S}(N), N \in K_{\lambda}$ in particular the realize/materialize problem which is here: if $N_{1} \leq_{\mathfrak{K}} N_{2}$ are in $K_{\lambda}, p_{\ell} \in \mathscr{S}\left(N_{\ell}\right)$ is minimal, $p_{1} \leq p_{2}$ are they conjugate? (i.e. $p_{2} \in \mathscr{S}_{p_{1}}\left(N_{2}\right)$ ).

7.2 Claim. If $N \in K_{\lambda}$ and $p \in \mathscr{S}(N)$ is minimal and reduced or just $p$ is reduced (see Definition 2.3(7)), then $p$ is inevitable.

Proof. Suppose $N, p$ form a counterexample. We can then find $N_{1}$ and $a$ such that $N \leq_{\mathfrak{K}} N_{1} \in K_{\lambda}, a \in N_{1} \backslash N$ and $p=\operatorname{tp}\left(a, N, N_{1}\right)$ and $\left(N, N_{1}, a\right)$ is reduced. As $p$ is not inevitable, there is $N_{2}$ such that: $N \leq_{\mathfrak{K}} N_{2} \in K_{\lambda}, N \neq N_{2}$ but no element of $N_{2}$ realizes $p$. By amalgamation in $K_{\lambda}$, without loss of generality there is $N_{3} \in K_{\lambda}$ such that $\ell \in\{1,2\} \Rightarrow N_{\ell} \leq_{\mathfrak{K}} N_{3}$. By 5.3 (i.e.7.1(e)) there is $q \in \mathscr{S}(N)$ which is inevitable so there are $c_{\ell} \in N_{\ell}$ with $q=\operatorname{tp}\left(c_{\ell}, N, N_{\ell}\right)$ for $\ell \in\{1,2\}$. By the equality of types (and amalgamation in $K_{\lambda}$ ) there is $N^{+} \in K$, a $\leq_{\mathfrak{K}^{-}}$ extension of $N_{1}$ and a $\leq_{\mathfrak{K}^{-}}$-embedding of $N_{2}$ into $N^{+}$over $N$ such that $f\left(c_{2}\right)=c_{1}$; so without loss of generality $N^{+}=N_{3}$ and $f$ is the identity, hence $c_{1}=c_{2}$. Now $a \notin N_{2}$ as $p=\operatorname{tp}\left(a, N, N_{1}\right)$ is not realized in $N_{2}$. So $\left(N, N_{1}, a\right) \leq\left(N_{2}, N_{3}, a\right)$ and $N_{2} \cap N_{1} \backslash N \neq \emptyset$ contradicting " $\left(N, N_{1}, a\right)$ is reduced".

7.3 Claim. 1) If $\kappa=c f(\kappa) \leq \lambda$ and $\bar{N}=\left\langle N_{i}: i \leq \omega \kappa\right\rangle$ is an $\leq_{\mathfrak{K} \text {-increasingly }}$ continuous sequence, $N_{i} \in K_{\lambda}, N_{i+1}$ universal over $N_{i}$, and $p \in \mathscr{\mathscr { S }}\left(N_{\omega \kappa}\right)$ is minimal reduced (or minimal inevitable) then for some $i<\omega \kappa$ we have $p\left\lceil N_{i} \in \mathscr{S}\left(N_{i}\right)\right.$ is minimal (so $p$ is the unique, non-algebraic extension of $p\left\lceil N_{i}\right.$ in $\mathscr{S}\left(N_{\omega \kappa}\right)$ (and of course, there is one)).

2) If $\lambda \geq \kappa=c f(\kappa), \bar{N}=\left\langle N_{i}: i \leq \kappa\right\rangle$ is $\leq_{\mathfrak{K}}$-increasing continuous in $K_{\lambda}$ and $p \in \mathscr{S}\left(N_{\kappa}\right)$ is minimal and reduced and the set $Y=:\left\{i<\kappa: N_{i+1}\right.$ is $(\lambda, \kappa)$-saturated over $\left.N_{i}\right\}$ is unbounded in $\kappa$ then for every large enough $i \in Y$ there is an isomorphism $f$ from $N_{i+1}$ onto $N_{\kappa}$ which is the identity on $N_{i}$ and $d^{5}$

(*) $f$ maps $p\left\lceil N_{i+1} \in \mathscr{S}\left(N_{i+1}\right)\right.$ to $p \in \mathscr{S}\left(N_{\kappa}\right)$.

\footnotetext{
5 in fact, this implies $(*)$
} 
Hence as $p$ is minimal reduced, so is $p\left\lceil N_{i+1}\right.$.

Proof. 1) We can choose $\left(N_{i}^{0}, N_{i}^{1}, a\right) \in K_{\lambda}^{3}$ for $i<\lambda^{+}$reduced, $\leq$-increasing continuous such that $N_{i}^{0} \neq N_{i+1}^{0}$. Let $N_{\ell}=\bigcup_{i<\lambda^{+}} N_{i}^{\ell}$. As in the proof of 5.5 for $c \in N_{1} \backslash N_{0}$

$$
I_{c}^{*}=\left\{j<\lambda^{+}: c \in N_{j}^{1} \text { and } \operatorname{tp}\left(c, N_{j}^{0}, N_{j}^{1}\right) \text { is minimal }\right\}
$$

is empty or is an end segment of $\lambda^{+}$and

$$
\begin{aligned}
E=\left\{\delta<\lambda^{+}:\right. & \text {if } c \in N_{\delta}^{1} \text { and } I_{c}^{*} \neq \emptyset \underline{\text { then }} I_{c}^{*} \cap \delta \\
& \text { is an unbounded subset of } \delta ; \text { and if } \alpha<\delta \\
& \text { then for some } \beta \in(\alpha, \delta), N_{\beta}^{0} \text { is universal over } N_{\alpha}^{0} \\
& \text { and if } \operatorname{Pr} \text { is one of the properties reduced and/or } \\
& \text { inevitable and/or minimal and there is } i \geq \delta \text { such that } \\
& \left(N_{i}^{0}, N_{i}^{1}, c\right) \text { has } \operatorname{Pr}, \text { then there are arbitrarily } \\
& \text { large such } i<\delta\}
\end{aligned}
$$

is a club of $\lambda^{+}$; for the universality demand in the definition of $E$ use categoricity in $\lambda^{+}$. Let $\delta \in \operatorname{acc}(\operatorname{acc}(E)), \operatorname{cf}(\delta)=\kappa$, let $\left\langle\alpha_{\zeta}: \zeta<\omega \kappa\right\rangle$ be an increasing continuous sequence of ordinals from $E$ with limit $\delta$, now set $\alpha_{\omega \kappa}=\delta$ and $N_{\zeta}^{\prime}=: N_{\alpha_{\zeta}}^{0}$.

So there is an isomorphism $f$ from $N_{\omega \kappa}$ onto $N_{\alpha_{\omega \kappa}}^{0}$ such that for every $\zeta<\omega \kappa$ we have $N_{\alpha_{2 \zeta}}^{0} \leq_{\mathfrak{K}} f\left(N_{\alpha_{2 \zeta}}\right) \leq_{\mathfrak{K}} N_{\alpha_{2 \zeta+1}}^{0}$ (so if $\zeta$ is a limit ordinal, then $N_{\alpha_{\zeta}}^{0}=N_{\alpha_{2 \zeta}}^{0}=$ $f\left(N_{\zeta}\right)$ ), so without loss of generality $f$ is the identity. As $p \in \mathscr{S}\left(N_{\omega \kappa}\right)$ is inevitable (by assumption or by 7.2) and $N_{\omega \kappa}=N_{\alpha_{\omega \kappa}}^{0}<_{\mathfrak{K}} N_{\alpha_{\omega \kappa}}^{1}$, for some $c \in N_{\alpha_{\omega \kappa}}^{1} \backslash N_{\alpha_{\omega \kappa}}^{0}$ we have $p=\operatorname{tp}\left(c, N_{\alpha_{\omega \kappa}}^{0}, N_{\alpha_{\omega_{\kappa}}}^{1}\right)$, so for some $\beta<\alpha_{\omega \kappa}$ we have $c \in N_{\beta}^{1}$. As $p$ is minimal (by assumption) clearly $\delta \in I_{c}$, but $\delta \in E$ so $\operatorname{Min}\left(I_{c}\right)<\delta$, but $I_{c}$ is an end segment of $\lambda^{+}$hence without loss of generality for some $\zeta<\omega \kappa$ we have $\beta=\alpha_{\zeta} \in I_{c}$. So for $\xi \in(\zeta, \omega \kappa)$, both $p \in \mathscr{S}\left(N_{\omega \kappa}\right)$ and $p\left\lceil N_{\xi} \in \mathscr{S}\left(N_{\xi}\right)\right.$ are non-algebraic extensions of the minimal $p\left\lceil N_{\alpha_{\zeta}}^{0} \in \mathscr{S}\left(N_{\alpha_{\zeta}}^{0}\right)\right.$ and $N_{\alpha_{\zeta}}^{0} \leq_{\mathfrak{K}} N_{\xi} \leq_{\mathfrak{K}} N_{\omega \kappa}$, all in $K_{\lambda}$, so we have proved part (1).

2) Without loss of generality every $\zeta<\kappa$ is in $Y$. We can find $\left\langle N_{\zeta}^{\prime}: \zeta \leq \omega \kappa\right\rangle$ as in part (1), moreover satisfying " $N_{\zeta+1}^{\prime}$ is $(\lambda, \kappa)$-saturated over $N_{\zeta}$ " and such that: for every $\zeta \leq \kappa$ we have $N_{\zeta}=N_{\omega \zeta}^{\prime}$. So again choose $\zeta<\kappa$ as there, we set $\beta=\alpha_{\omega \zeta} \in I_{c}^{*}$. If $\xi \in Y \quad \& \omega \xi>\zeta$ clearly by the uniqueness of $(\lambda, \kappa)$ saturated models there is an isomorphic $f$ from $N_{\xi+1}=N_{\omega(\xi+1)}^{\prime}$ onto $N_{\kappa}=N_{\omega \kappa}^{\prime}$ over $N_{\xi}=N_{\omega \kappa}^{\prime}$, and $f\left(p\left\lceil N_{\xi+1}\right)=p\right.$ is proved as above. 
7.4 Claim. 1) If $M_{0} \leq_{\mathfrak{K}} M_{1}$ are in $K_{\lambda}$ and the types $p_{\ell} \in \mathscr{S}\left(M_{\ell}\right)$ are minimal reduced, for $\ell=0,1$ and $p_{0}=p_{1} \uparrow M_{0}$ then $p_{0}, p_{1}$ are conjugate; (i.e. there is an isomorphic $f$ from $M_{0}$ onto $M_{1}$ such that $\left.f\left(p_{0}\right)=p_{1}\right)$.

2) If in addition $M \leq_{\mathfrak{K}} M_{0}$ and $M_{0}, M_{1}$ are $(\lambda, \kappa)$-saturated over $M$, then $p_{0}, p_{1}$ are conjugate over $N$.

Remark. Note that $p$ minimal (or reduced) implies that $p$ is not algebraic.

Proof. 1) Let $\left\langle\left(N_{i}^{0}, N_{i}^{1}, a\right): i<\lambda^{+}\right\rangle$and $E$ be as in the proof of 7.3 and $\kappa=\operatorname{cf}(\kappa) \leq \lambda$. For each $\delta \in S_{\kappa}=:\left\{\alpha<\lambda^{+}: \alpha \in E\right.$ and $\left.\operatorname{cf}(\alpha)=\kappa\right\}$, and minimal reduced $p \in \mathscr{S}\left(N_{\delta}^{0}\right)$, we know that for some $i_{p}<\delta, p\left\lceil N_{i_{p}}^{0}\right.$ is minimal reduced [why? by 7.3(1),(2)] and some $q_{p} \in \mathscr{S}\left(N_{i_{p}}^{0}\right)$ is conjugate to $p$. For $\kappa=\operatorname{cf}(\kappa) \leq \lambda, q \in \mathscr{S}\left(N_{i}^{0}\right), i<\lambda^{+}, r \in \mathscr{S}\left(N_{i}^{0}\right)$ minimal let $A_{q, r}^{\kappa, i}=\left\{\delta<\lambda^{+}\right.$: there is a type $p$ such that $r \subseteq p \in \mathscr{S}\left(N_{\delta}^{0}\right), p$ non-algebraic (this determines $p$ ), $p$ minimal reduced, $i_{p}=i, q_{p}=q$ (and clearly $\left.p\left\lceil N_{i}^{0}=r\right)\right\}$.

Next let

$$
\begin{aligned}
E_{1}=\left\{\delta<\lambda^{+}\right. & \text {for every } \kappa=\operatorname{cf}(\kappa) \leq \lambda, \\
& r, q \in \mathscr{S}\left(N_{i}^{0}\right) \text { and } i<\delta, \text { if } A_{q, r}^{\kappa, i} \text { is well defined and } \\
& \text { unbounded in } \left.\lambda^{+} \underline{\text { then }} \text { it is unbounded in } \delta\right\} .
\end{aligned}
$$

So if $\delta_{1} \in E_{1}, \kappa=\operatorname{cf}\left(\delta_{1}\right), p_{1} \in \mathscr{S}\left(N_{\delta}^{0}\right)$ is minimal reduced, $\underline{\text { then }}$ we can find $\delta_{0}<\delta_{1}, \operatorname{cf}\left(\delta_{0}\right)=\kappa$, and $p_{0} \in \mathscr{S}\left(N_{\delta_{0}}^{0}\right)$ minimal reduced with $q_{p_{1}}=q_{p_{0}}, i_{p_{1}}=i_{p_{0}}, p_{0} \uparrow N_{i_{p_{0}}}^{0}=p_{1} \uparrow N_{i_{p_{1}}}^{0}$ call it $r$, it is necessarily minimal.

As $p_{1}, p_{0}$ extend $r, N_{i_{p_{0}}}^{0}=N_{i_{p_{1}}}^{0} \leq_{\mathfrak{K}} N_{\delta_{0}}^{0} \leq_{\mathfrak{K}} N_{\delta_{1}}^{0}$, necessarily $p_{1}=p_{0}\left\lceil N_{\delta_{0}}^{0}\right.$, and also they are both conjugate to $q_{p_{0}}=q_{p_{1}}$ hence they are conjugate.

Next we prove

$(*)$ if $M_{0}<_{\mathfrak{K}} M_{1}$ are in $K_{\lambda}, M_{1}$ is $(\lambda, \kappa)$-limit over $M_{0}, p_{0}^{\prime} \in \mathscr{S}\left(M_{0}\right)$ is minimal reduced and $p_{0}^{\prime} \leq p_{1}^{\prime} \in \mathscr{S}\left(M_{1}\right), p_{1}^{\prime}$ non-algebraic, then $p_{0}^{\prime}, p_{1}^{\prime}$ are conjugate.

Above we have a good amount of free choice in choosing $p_{1} \in \mathscr{S}\left(N_{\delta_{1}}^{0}\right)$ (it should be minimal and reduced) so we could have chosen $p_{1}$ to be conjugate to $p_{0}^{\prime}$ (i.e. is in $\mathscr{S}_{p_{0}^{\prime}}\left(N_{\delta_{1}}^{0}\right)$; now also the corresponding $p_{0}$ is conjugate to $p_{1}$ hence $p_{0}$ is conjugate to $p_{0}^{\prime}$, hence we can find an isomorphism $f_{0}$ from $M_{0}$ onto $N_{\delta_{0}}^{0}, f_{0}\left(p_{0}^{\prime}\right)=p_{0}$, and extend it to an isomorphism $f_{1}$ from $M_{1}$ onto $N_{\delta_{1}}^{0}$, so necessarily $f\left(p_{1}^{\prime}\right)=p_{1}$ (as $p_{1}$ is the unique non-algebraic extension). As $p_{0}, p_{1}$ are conjugate through $f_{1}$ also $p_{0}^{\prime}, p_{1}^{\prime}$ are conjugate. So $(*)$ holds.

Now assume just $M_{0} \leq_{\mathfrak{K}} M_{1}$ are in $K_{\lambda}, p_{0} \in \mathscr{S}\left(M_{0}\right)$ minimal reduced, $p_{1} \in \mathscr{S}\left(M_{1}\right)$ the unique non-algebraic extension of $p_{0}$ and it is reduced (and necessarily minimal). There is $M_{2}, M_{1} \leq_{\mathfrak{K}} M_{2} \in K_{\lambda}, M_{2}$ is $(\lambda, \kappa)$-limit over $M_{1}$ hence also over $M_{0}$ 
and let $p_{2}$ be the unique non-algebraic extension of $p_{1}$ in $\mathscr{S}\left(M_{2}\right)$ hence $p_{2}$ is also the unique non-algebraic extension of $p_{0}$ in $\mathscr{S}\left(M_{2}\right)$.

Using $(*)$ on $\left(M_{0}, M_{2}, p_{0}, p_{2}\right)$ and on $\left(M_{1}, M_{2}, p_{1}, p_{2}\right)$ and get that $p_{0}, p_{2}$ are conjugate and that $p_{1}, p_{2}$ are conjugate hence $p_{1}, p_{2}$ are conjugate, the required result.

2) Similar proof.

$\square_{7.4}$

7.5 Claim. 1) Assume $M_{1} \leq_{\mathfrak{K}} M_{2}$ are in $K_{\lambda}$ and $M_{2}$ is $(\lambda, \kappa)$-saturated over $M_{1}$. If $p_{1} \in \mathscr{S}\left(M_{1}\right)$ is minimal and reduced, then $p_{2}$, the unique non-algebraic extension of $p_{1}$ in $\mathscr{S}\left(M_{2}\right)$, is reduced (and, of course, minimal).

2) There is no need to assume " $p_{1}$ reduced.

Proof. 1) We can find $\left\langle N_{i}: i \leq \kappa\right\rangle$, an $\leq_{\mathfrak{K}}$-increasingly continuous sequence in $K_{\lambda}$ such that $N_{i+1}$ is $(\lambda, \kappa)$-saturated over $N_{i}$ and $N_{\kappa}=M_{1}$. So by 7.3(1),(2), for some $\zeta<\kappa$ we have: $p_{2}\left\lceil N_{\zeta}\right.$ is minimal and for some isomorphism $f$ from $N_{\zeta+1}$ onto $N_{\kappa}$ we have $f\left(p_{1}\left\lceil N_{\zeta}\right)=p_{1}\right.$ and $f\left\lceil N_{\zeta}=\operatorname{id}_{N_{\zeta}}\right.$. Also $M_{1}, N \zeta+1$ are isomorphic over $N_{\zeta}$ (as both are $(\lambda, \kappa)$-saturated over it) hence there is an isomorphism $g$ from $N_{\zeta+1}$ onto $M_{2}$ over $N_{\zeta}$. Now $p_{1}=f\left(p_{1}\left\lceil N_{\zeta+1}\right)\right.$ and $f_{2}=: g\left(p_{1}\left\lceil N_{\zeta+1}\right)\right.$ are non-algebraic extensions of $p_{1}\left\lceil N_{\zeta}\right.$ which is minimal, hence $p_{1}=p_{2}\left\lceil M_{1}\right.$ and $p_{2}$ is as mentioned in 7.5. Now $g \circ f^{-1}$ show that $p_{1}, p_{2}$ are conjugate so as $p_{1}$ is reduced also $p_{2}$ is reduced.

2) Easy as we can find $N, M_{1} \leq_{K} N, q \in \mathscr{S}(N)$ minimal reduced; without loss of generality $N \leq_{\mathfrak{K}} M_{2}$ and $M_{2}$ is $(\lambda, \kappa)$-saturated over $N$, and apply part (1). $\square_{7.5}$

7.6 Claim. Assume

(a) $N_{i, j} \in K_{\lambda}$ for $i \leq \delta_{1}, j \leq \delta_{2}$

(b) $\left\langle N_{i, j}: j \leq \delta_{2}\right\rangle$ is $\leq_{\mathfrak{K}}$-increasingly continuous for each $i \leq \delta_{1}$

(c) $\left\langle N_{i, j}: i<\delta_{1}\right\rangle$ is $\leq_{\mathfrak{K}}$-increasingly continuous for each $j \leq \delta_{2}$

(d) $\left\langle N_{i, j}: i \leq \delta_{1}, j \in \delta_{2}\right\rangle$ is smooth, i.e. $N_{i_{1}, j_{1}} \cap N_{i_{2}, j_{2}}=N_{\min \left\{i_{1}, i_{2}\right\}} \cap N_{\min \left\{j_{1}, j_{2}\right\}}$

(e) $N_{i+1, j+1}$ is universal over $N_{i, j+1} \cup N_{i+1, j}$ (i.e. $N_{i+1, j+1}$ is universal over some $N_{i+1, j+1}^{\prime}$ where $N_{i, j+1} \cup N_{i+1, j} \subseteq N_{i+1, j+1}^{\prime}$,

(f) $\delta_{1}$ is divisible by $\operatorname{cf}\left(\delta_{2}\right) \times \lambda \times \omega$ (and even easier if $\delta_{1}=1$ !).

Then $N_{\delta_{1}, \delta_{2}}$ is $\left(\lambda, c f\left(\delta_{1}\right)\right)$-saturated over $N_{i, \delta_{1}}$ for $i<\delta_{1}$.

Proof. Without loss of generality $\delta_{2}=\operatorname{cf}\left(\delta_{2}\right)$. (Why? let $\left\langle\alpha_{\varepsilon}: \varepsilon \leq \operatorname{cf}\left(\delta_{1}\right)\right\rangle$ be increasingly continuous with limit $\delta_{1}$ such that $\left[\varepsilon\right.$ limit $\leftrightarrow \alpha_{\varepsilon}$ limit], and use $\left.N_{i, \varepsilon}^{\prime}=N_{i, \alpha_{\varepsilon}}\right)$.

For $i<\delta_{1}, j<\delta_{2}$ let $M_{i, j}, M_{i, j}^{\prime}$ be such that $M_{i+1, j} \cup M_{i, j+1} \subseteq M_{i, j} \leq_{\mathfrak{K}} M_{i, j}^{\prime} \leq$ $M_{i+1, j+1}$ and $M_{i, j}^{\prime}$ is $(\lambda, \operatorname{cf}(\lambda))$-saturated over $M_{i, j}$.

Now let $p \in \mathscr{S}(N)$ be minimal and reduced; and for $i \leq \delta_{1}, j \leq \delta_{2}$ let $p_{i, j} \in \mathscr{S}\left(N_{i, j}\right)$ be the unique non-algebraic extension of $p$ in $\mathscr{S}\left(N_{i, j}\right)$ so it is minimal. Now for $i<$

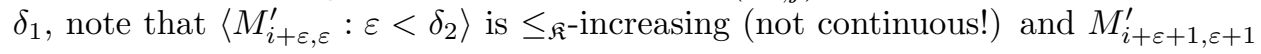
is $(\lambda, \operatorname{cf}(\lambda))$-saturated over $M_{i+\varepsilon, \varepsilon}^{\prime}$ and $\bigcup_{\varepsilon<\delta} M_{i+\varepsilon, \varepsilon}^{\prime}=\bigcup_{\varepsilon<\delta_{1}} N_{i+1, \varepsilon+1}=N_{i+\delta_{2}, \delta_{2}}$, and 
$N_{0,0} \leq_{\mathfrak{k}} M_{1,0}^{\prime}$, hence by 7.5 we know that $p_{i+\delta_{2}, \delta_{2}}$ is reduced (and minimal). In fact, similarly $\alpha<\delta_{1} \& \operatorname{cf}(\alpha)=\operatorname{cf}\left(\delta_{2}\right) \Rightarrow p_{\alpha, \delta_{2}}$ is reduced. As $N_{i+1, j+1} \neq$ $N_{i+1, j} \cup N_{i, j+1}$ and clause (d) (smoothness) necessarily $N_{i+\delta_{2}, \delta_{2}}<N_{i+\delta_{2}+1, \delta_{2}}$, hence some $c \in N_{i+\delta_{2}+i, \delta_{2}} \backslash N_{i+\delta_{2}, \delta_{2}}$ realizes $p_{i+\delta_{2}, \delta_{2}}$. So if $\alpha \leq \delta_{1}$ is divisible by $\delta_{2} \times \lambda$ and has cofinality $\operatorname{cf}\left(\delta_{2}\right)$ and $\beta<\alpha$, then by $5.6 N_{\alpha, \delta_{2}}$ is universal over $N_{\beta, \delta_{2}}$. As $\delta_{1}$ is divisible by $\operatorname{cf}\left(\delta_{2}\right) \times \lambda \times \omega$ we are done.

7.7 Lemma. 1) For every $N \in K_{\lambda^{+}}$we can find a representation $\bar{N}=\left\langle N_{i}: i<\lambda^{+}\right\rangle$, with $N_{i+1}$ being $(\lambda, c f(\lambda))$-saturated over $N_{i}$.

2) If for $\ell=1,2$ we have $N^{\ell}=\left\langle N_{i}^{\ell}: i\left\langle\lambda^{+}\right\rangle\right.$as in part (1) then there is an isomorphism $f$ from $N^{1}$ onto $N^{2}$ mapping $N_{i}^{1}$ onto $N_{i}^{2}$ for each $i<\lambda^{+}$. Moreover for any $i<\lambda^{+}$and isomorphism $g$ from $N_{i}^{1}$ onto $N_{i}^{2}$ we can find an isomorphism $f$ from $N^{1}$ onto $N^{2}$ extending $g$ and mapping $N_{j}^{1}$ onto $N_{j}^{2}$ for each $j \in\left[i, \lambda^{+}\right)$.

3) If $N^{0} \leq_{\mathfrak{K}} N^{1}$ are in $K_{\lambda^{+}}$then we can find representation $\bar{N}^{\ell}$ of $N^{\ell}$ as in (1) with $N_{i}^{0}=N^{0} \cap N_{i}^{1}$, (so $\left.N_{i}^{0} \leq_{\mathfrak{K}} N_{i}^{1}\right)$.

4) For any strictly increasing function $\mathbf{f}: \lambda^{+} \rightarrow \lambda^{+}$, we can find $N_{i, \varepsilon}$ for $i<\lambda^{+}, \varepsilon \leq \lambda \times(1+\mathbf{f}(i))$ such that:

(a) $N_{i, \varepsilon} \in K_{\lambda}$

(b) $\left\langle N_{i, \varepsilon}: \varepsilon \leq \lambda \times(1+\mathbf{f}(i))\right\rangle$ is strictly $\leq_{\mathfrak{K}^{-}}$-increasing continuous

(c) for each $\varepsilon,\left\langle N_{i, \varepsilon}: i \in\left[i_{\varepsilon}, \lambda^{+}\right)\right\rangle$is a representation as in (1) where $i_{\varepsilon}=\operatorname{Min}\{i: \varepsilon \leq \lambda \times(1+\mathbf{f}(i))\}$

(d) if $\varepsilon<\lambda \times(1+f(i))$ and $i<j<\lambda^{+}$then $N_{j, \varepsilon} \cap N_{i, \lambda \times(1+\mathbf{f}(i))}=N_{i, \varepsilon}$

(e) $N_{i+1, \varepsilon+1}$ is $\left(\lambda, \aleph_{0}\right)$-saturated over $N_{i+1, \varepsilon} \cup N_{i, \varepsilon+1}$.

Proof. Straight.

4) First use $\mathbf{f}^{\prime}: \lambda^{+} \rightarrow \lambda^{+}$which is $\mathbf{f}^{\prime}(i)=\lambda^{\omega} \times \mathbf{f}(i)$. Then define the $N_{i, \varepsilon}(\varepsilon<$ $\lambda \times\left(1+\mathbf{f}^{\prime}(i) ; i<\lambda^{+}\right)$. "Forget" about " $N_{i+1, \varepsilon}$ is $(\lambda, \operatorname{cf}(\lambda))$-saturated over $N_{i, \varepsilon}$ ", remember we have disjoint amalgamation by 5.11. Now by 7.6, even for $\varepsilon$ limit divisible by $\lambda^{3}$ we get $N_{i+\lambda, \varepsilon}$ is $(\lambda, \operatorname{cf}(\lambda))$-saturated over $N_{i, \varepsilon}$, so renaming all is O.K.).

We can deduce from 7.5, but to keep the door open to other uses we shall not use

7.8 Claim. If $\kappa_{\ell}=c f\left(\kappa_{\ell}\right) \leq \lambda$, and $N_{\ell}$ is $\left(\lambda, \kappa_{\ell}\right)$-saturated over $N$ for $\ell=1,2$ then $N_{1}, N_{2}$ are isomorphic over $N$.

Proof. We can define by induction on $i \leq \lambda \times \kappa_{1}$, and then by induction on $j \leq \lambda \times \kappa_{2}, M_{i, j}$ such that:

(a) $M_{i, j} \in K_{\lambda}$

(b) $M_{0,0}=N$

(c) $i_{1} \leq i \& j_{1} \leq j \Rightarrow M_{i_{1}, j_{1}} \leq_{\mathfrak{K}} M_{i, j}$

(d) $M_{i_{1}, j_{1}} \cap M_{i_{2}, j_{2}}=M_{\min \left\{i_{1}, i_{2}\right\}, \min \left\{j_{1}, j_{2}\right\}}$

(e) $M_{i, j}$ is $\leq_{\mathfrak{K}^{-}}$-increasing continuous in $i$

(f) $M_{i, j}$ is $<_{\mathfrak{K}}$-increasing continuous in $j$ 
(g) $M_{0, j} \neq M_{0, j+1}$

(h) $M_{i+1,0} \neq M_{i, 0}$

(i) $M_{i+1, j+1}$ is universal over $M_{i+1, j} \cup M_{i, j+1}$.

There is no problem by 5.11(1) (using the existence of disjoint amalgamation).

Now $M_{\lambda \times \kappa_{1}, \lambda \times \kappa_{2}}$ is the union of the strictly $<_{\mathfrak{K}}$-increasing sequence

$\left\langle M_{0,0}\right\rangle^{\wedge}\left\langle M_{\lambda \times i, \lambda \times \kappa_{2}}: i<\kappa_{1}\right\rangle$ hence by 7.6 is $\left(\lambda, \kappa_{1}\right)$-saturated over $M_{0,0}=N$ hence $M_{\lambda \times \kappa_{1}, \lambda \times \kappa_{2}} \cong_{N} N_{1}$. Similarly $M_{\lambda \times \kappa_{1}, \lambda \times \kappa_{2}}$ is the union of the strictly $\leq_{\mathfrak{K}^{-}}$-increasing sequence $\left\langle M_{0,0}\right\rangle^{\wedge}\left\langle M_{\lambda \times \kappa_{1}, \lambda \times j}: j<\kappa_{2}\right\rangle$ hence is $\left(\lambda, \kappa_{2}\right)$-saturated over $M_{0,0}=N$, hence $M_{\lambda \times \kappa_{1}, \lambda \times \kappa_{2}} \cong_{N} N_{2}$. Together $N_{1}, N_{2}$ are isomorphic over $N$. 


\section{$\S 8$ UNIQUENESS OF AMALGAMATION IN $\mathfrak{K}_{\lambda}$}

We deal in this section only with $K_{\lambda}$.

We want to, at least, approximate unique amalgamation using as starting point $\otimes$ of 6.4 (see also 6.7 ), i.e. $K_{\lambda}^{3, \text { uq }} \neq \emptyset$.

8.1 Hypothesis. 1) Assume hypothesis 7.1, so

(a) $\mathfrak{K}$ abstract elementary class

(b) $\mathfrak{K}$ has amalgamation in $\lambda$

(c) $\mathfrak{K}$ is categorical in $\lambda$ (can be weakened)

(d) $\mathfrak{K}$ is stable in $\lambda$ (see 5.7 , clause $(a)$ )

(e) there is an inevitable $p \in \mathscr{S}(N)$ for $N \in K_{\lambda}$ (holds by 5.3)

$(f)$ the basic properties in type theory.

2) $\left(M^{*}, N^{*}\right)$ is some pair in

$K_{\lambda}^{3, \text { uq }}=\left\{\left(M_{0}, M_{2}\right): M_{0} \leq_{\mathfrak{K}} M_{2}\right.$ are in $K_{\lambda}$ and for every $M_{1}, M_{0} \leq_{\mathfrak{K}} M_{1} \in K_{\lambda} \Rightarrow$ $\left(M_{0}, M_{1}, M_{2}\right) \in K_{\lambda}^{\text {uniq }}$; equivalently for some $M_{1},\left(M_{0}, M_{1}, M_{2}\right)$ are as in $\bigotimes$ of 6.4$\}$ (eventually the choice does not matter; if each time instead of $\cong\left(M^{*}, N^{*}\right)$ we write $\in K_{\lambda}^{2 \text {,uq }}$, see 8.11; but if we start with this definition then the uniqueness theorems will be more cumbersome).

8.2 Definition. Assume $\bar{\delta}=\left\langle\delta_{1}, \delta_{2}, \delta_{3}\right\rangle, \delta_{2}$ a limit ordinal $<\lambda^{+}$but $\delta_{1}, \delta_{3}$ are $<\lambda^{+}$ and may be 1 . We say that $N F_{\lambda, \bar{\delta}}\left(N_{0}, N_{1}, N_{2}, N_{3}\right)$ (we say $N_{1}, N_{2}$ are saturated and smoothly amalgamated in $N_{3}$ over $N_{0}$ for $\bar{\delta}$ ) when:

(a) $N_{\ell} \in K_{\lambda}$ for $\ell \in\{0,1,2,3\}$

(b) $N_{0} \leq_{\mathfrak{K}} N_{\ell} \leq_{\mathfrak{K}} N_{3}$ for $\ell=1,2$

(c) $N_{1} \cap N_{2}=N_{0}$ (i.e. in disjoint amalgamation)

(d) $N_{1}$ is $\left(\lambda, \operatorname{cf}\left(\delta_{1}\right)\right)$-saturated over $N_{0}$

(e) $N_{2}$ is $\left(\lambda, \operatorname{cf}\left(\delta_{2}\right)\right)$-saturated over $N_{0}$, if $\delta_{1}=1$ this means just $N_{0} \leq_{\mathfrak{K}} N_{2}$

(f) there are $N_{1, i}, N_{2, i}$ for $i \leq \lambda \times \delta_{1}$ (called the witness) such that:

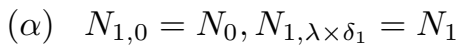

(в) $N_{2,0}=N_{2}$

( $\gamma)\left\langle N_{\ell, i}: i \leq \lambda \times \delta_{1}\right\rangle$ is $<_{\mathfrak{K}}$-increasing continuous for $\ell=1,2$

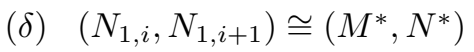

(ع) $N_{2, i} \cap N_{1}=N_{1, i}$

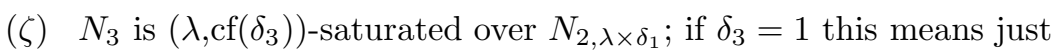
$N_{2, \lambda \times \delta_{1}} \leq_{\mathfrak{K}} N_{3}$

Discussion: Why this definition of NF? We need a nonforking notion with the usual properties. We first describe a version depending on $\left\langle\delta_{0}, \delta_{1}, \delta_{2}\right\rangle$ and get $N F=$ $N F_{\lambda, \bar{\delta}}, \bar{\delta}$ works like a scaffold - eventually $\bar{\delta}$ disappears. 
8.3 Definition. 1) We say $N_{1} \bigcup_{N_{0}}^{N_{3}} N_{2}$ (or $N_{1}, N_{2}$ are smoothly amalgamated over $N_{0}$ inside $N_{3}$ or $N F_{\lambda}\left(N_{0}, N_{1}, N_{2}, N_{3}\right)$ ) if we can find $M_{\ell} \in K_{\lambda}$ (for $\ell<4$ ) such that:

(a) $N F_{\lambda,\langle\lambda, \lambda, \lambda\rangle}\left(M_{0}, M_{1}, M_{2}, M_{3}\right)$

(b) $N_{\ell} \leq_{\mathfrak{K}} M_{\ell}$ for $\ell<4$

(c) $N_{0}=M_{0}$

(d) $M_{1}, M_{2}$ are $\left(\lambda, \operatorname{cf}(\lambda)\right.$ )-saturated over $N_{0}$ (follows by (a) see clauses (d), (e) of 8.2).

8.4 Claim. 1) If $\bar{\delta}=\left\langle\delta_{1}, \delta_{2}, \delta_{3}\right\rangle$, $\delta_{\ell}$ a limit ordinal $<\lambda^{+}$and $N_{\ell} \in K_{\lambda}$ for $\ell<3$, and $N_{1}$ is $\left(\lambda, c f\left(\delta_{1}\right)\right)$ - saturated over $N_{0}$ and $N_{2}$ is $\left(\lambda, c f\left(\delta_{2}\right)\right)$-saturated over $N_{0}$ and $N_{0} \leq_{\mathfrak{K}} N_{1}, N_{0} \leq_{\mathfrak{K}} N_{2}$ and for simplicity $N_{1} \cap N_{2}=N_{0}$. Then we can find $N_{3}$ such that $N F_{\lambda, \bar{\delta}}\left(N_{0}, N_{1}, N_{2}, N_{3}\right)$.

2) Moreover, we can choose any $\left\langle N_{1, i}: i \leq \lambda \times \delta_{1}\right\rangle$ as in $8.2(f)(\alpha),(\gamma),(\delta)$ as part of the witness.

Proof. Straight (remembering 7.5 (and uniqueness of the $\left(\lambda, \operatorname{cf}\left(\delta_{1}\right)\right)$-saturated model over $\left.N_{0}\right)$ ).

8.5 Claim. If Definition 8.2, if $\delta_{3}$ is a limit ordinal, then without loss of generality (even without changing $\left\langle N_{1, i}: i \leq \lambda \times \delta_{1}\right\rangle$ )

(g) $N_{2, i+1}$ is $\left(\lambda, c f\left(\delta_{2}\right)\right.$ )-saturated over $N_{i+1}^{1} \cup N_{i}^{2}$ (which means it is $\left(\lambda, c f\left(\delta_{2}\right)\right)$-saturated over some $N$, where $\left.N_{i+1}^{1} \cup N_{i}^{2} \subseteq N \leq_{\mathfrak{K}} N_{2, i+1}\right)$.

Proof. So assume $N F_{\lambda, \bar{\delta}}\left(N_{0}, N_{1}, N_{2}, N_{3}\right)$ holds as witnessed by $\left\langle N_{\ell, i}: i \leq \lambda \times \delta_{\ell}\right\rangle$ for $\ell=1,2$. Now we choose by induction on $i \leq \lambda \times \delta_{1}$ a model $M_{2, i} \in K_{\lambda}$ such that:

(i) $N_{2, i} \leq M_{2, i}$

(ii) $M_{2,0}=N_{2}$

(iii) $M_{2, i}$ is $\leq_{\mathfrak{K}}$-increasing continuous

(iv) $M_{2, i} \cap N_{2, \lambda \times \delta_{1}}=N_{2, i}$ moreover $M_{2, i} \cap N_{3}=N_{2, i}$

(v) $M_{2, i+1}$ is $\left(\lambda, \operatorname{cf}\left(\delta_{2}\right)\right)$-saturated over $M_{2, i} \cup N_{2, i+1}$.

There is no problem to carry the definition. Let $M_{3}$ be such that $M_{2, \lambda \times \delta_{1}} \leq_{\mathfrak{K}}$ $M_{3} \in K_{\lambda}$ and $M_{3}$ is $\left(\lambda, \operatorname{cf}\left(\delta_{3}\right)\right)$-saturated over $M_{2, \lambda \times \delta_{1}}$. So both $M_{3}$ and $N_{3}$ are $\left(\lambda, \operatorname{cf}\left(\delta_{3}\right)\right)$-saturated over $N_{2, \lambda \times \delta_{1}}$, hence they are isomorphic over $N_{2, \lambda \times \delta_{1}}$ so let $f$ be an isomorphism from $M_{3}$ onto $N_{3}$ which is the identity over $N_{2, \lambda \times \delta_{1}}$. Clearly $\left\langle N_{1, i}: i \leq \lambda \times \delta_{1}\right\rangle,\left\langle f\left(M_{2, i}\right): i \leq \lambda \times \delta_{1}\right\rangle$ are also witnesses for $N F_{\lambda, \bar{\delta}}\left(N_{0}, N_{1}, N_{2}, N_{3}\right)$ satisfying the extra demand $(g)$. 
8.6 Claim. (Weak Uniqueness).

Assume that for $x \in\{a, b\}$, we have $N F_{\lambda, \bar{\delta}^{x}}\left(N_{0}^{x}, N_{1}^{x}, N_{2}^{x}, N_{3}^{x}\right)$ holds as witnessed by $\left\langle N_{1, i}^{x}: i \leq \lambda \times \delta_{1}^{x}\right\rangle,\left\langle N_{2, i}^{x}: i \leq \lambda \times \delta_{1}^{x}\right\rangle$ and $\delta_{1}=: \delta_{1}^{a}=\delta_{1}^{b}, c f\left(\delta_{2}^{a}\right)=c f\left(\delta_{2}^{b}\right)$ and $c f\left(\delta_{3}^{a}\right)=c f\left(\delta_{3}^{b}\right) \geq \aleph_{0}$.

Suppose further that $f_{\ell}$ is an isomorphism from $N_{\ell}^{a}$ onto $N_{\ell}^{b}$ for $\ell=0,1,2$, moreover: $f_{0} \subseteq f_{1}, f_{0} \subseteq f_{2}$ and $f_{1}\left(N_{1, i}^{a}\right)=N_{1, i}^{b}$.

Then we can find an isomorphism $f$ from $N_{3}^{a}$ onto $N_{3}^{b}$ extending $f_{1} \cup f_{2}$.

Proof. Without loss of generality $N_{2, i+1}^{x}$ is $\left(\lambda, \operatorname{cf}\left(\delta_{2}\right)\right)$-saturated over $N_{1, i+1}^{x} \cup N_{2, i}^{x}$ (by 8.5, note "without changing the $N_{1, i}$ 's). Now we choose by induction on $i \leq \lambda \times \delta_{1}$ an isomorphism $g_{i}$ from $N_{2, i}^{a}$ onto $N_{2, i}^{b}$ such that: $g_{i}$ is increasing in $i$ and $g_{i}$ extends $\left(f_{1}\left\lceil N_{1, i}^{a}\right) \cup f_{2}\right.$.

For $i=0$ choose $g_{0}=f_{2}$ and for $i$ limit let $g_{i}$ be $\bigcup_{j<i} g_{j}$ and for $i=j+1$ use $\left(N_{1, i}, N_{1, i+1}\right) \cong\left(M^{*}, N^{*}\right)$ (see 8.2) and the extra saturation clause (g). Now we can extend $g_{\lambda \times \delta_{1}}$ to an isomorphism from $N_{3}^{a}$ onto $N_{3}^{b}$ as $N_{3}^{x}$ is $\left(\lambda, \operatorname{cf}\left(\delta_{3}\right)\right)$-saturated from $N_{2, \lambda \times \delta_{1}}^{x}$ (for $x \in\{a, b\}$ ); note that knowing 8.6 possibly the choice of $\left\langle N_{1, i}: i \leq \lambda \times \delta_{1}\right\rangle$ matters.

Now we prove an "inverted" uniqueness

8.7 Claim. Suppose that

(a) for $x \in\{a, b\}$ we have $N F_{\lambda, \bar{\delta}^{x}}\left(N_{0}^{x}, N_{1}^{x}, N_{2}^{x}, N_{3}^{x}\right)$

(b) $\bar{\delta}^{x}=\left\langle\delta_{1}^{x}, \delta_{2}^{x}, \delta_{3}^{x}\right\rangle, \delta_{1}^{a}=\delta_{2}^{b}, \delta_{2}^{a}=\delta_{1}^{b}, c f\left(\delta_{3}^{a}\right)=c f\left(\delta_{3}^{b}\right)$ all limit ordinals

(c) $f_{0}$ is an isomorphism from $N_{0}^{a}$ onto $N_{0}^{b}$

(d) $f_{1}$ is an isomorphism from $N_{1}^{a}$ onto $N_{2}^{b}$

(e) $f_{2}$ is an isomorphism from $N_{2}^{a}$ onto $N_{1}^{b}$

(f) $f_{0} \subseteq f_{1}$ and $f_{0} \subseteq f_{2}$.

Then there is an isomorphism from $N_{3}^{a}$ onto $N_{3}^{b}$ extending $f_{1} \cup f_{2}$.

Before proving

8.8 Subclaim. 1) For any limit ordinals $\delta_{1}^{a}, \delta_{2}^{a}, \delta_{3}^{a} \leq \lambda$ we can find $M_{i, j}$ (for $i \leq \lambda \times \delta_{1}^{a}$ and $\left.j \leq \lambda \times \delta_{2}^{a}\right)$ and $M_{3}$ such that:

(A) $M_{i, j} \in K_{\lambda}$

(B) $i_{1} \leq i_{2} \& j_{1} \leq j_{2} \Rightarrow M_{i_{1}, j_{1}} \leq_{\mathfrak{K}} M_{i_{2}, j_{2}}$

(C) if $i \leq \lambda \times \delta_{1}$ is a limit ordinal and $j \leq \lambda \times \delta_{2}$ then $M_{i, j}=\bigcup_{\zeta<i} M_{\zeta, j}$

(D) if $i \leq \lambda \times \delta_{1}$ and $j \leq \lambda \times \delta_{2}$ is a limit ordinal then $M_{i, j}=\bigcup_{\xi<j} M_{i, \xi}$

(E) $\left(M_{0, j}, M_{0, j+1}\right) \cong\left(M^{*}, N^{*}\right)$

(F) $\left(M_{i, 0}, M_{i+1,0}\right) \cong\left(M^{*}, N^{*}\right)$ 
(G) for $i_{1}, i_{2} \leq \lambda \times \delta_{1}$ and $j_{1}, j_{2} \leq \lambda \times \delta_{2}$ we have $M_{i_{1}, j_{1}} \cap M_{i_{2}, j_{2}}=M_{\min \left\{i_{1}, i_{2}\right\}, \min \left\{j_{1}, j_{2}\right\}}$,

(H) $M_{\lambda \times \delta_{1}, \lambda \times \delta_{2}} \leq_{\mathfrak{K}} M_{3} \in K_{\lambda}$ moreover $M_{3}$ is $\left(\lambda, c f\left(\delta_{3}\right)\right)$-saturated over $M_{\lambda \times \delta_{1}, \lambda \times \delta_{2}}$.

2) Moreover, it is O.K. if $\left\langle M_{0, j}: j \leq \lambda \times \delta_{2}^{a}\right\rangle,\left\langle M_{i, 0}: i \leq \lambda \times \delta_{1}^{a}\right\rangle$ are pregiven as long as both $\leq_{\mathfrak{K}}$-increasing continuous in $\mathfrak{K}_{\lambda}$ satisfying $(E)+(F)$ and $M_{0, \lambda \times \delta_{2}^{a}} \cap$ $M_{\lambda \times \delta_{2}^{a}, 0}=M_{0,0}$.

Proof. 1) This is done by induction on $i$ and for fixed $i$ by induction on $j$. For $i=0, j=0$ let $M_{0,0} \in K_{\lambda}$ be arbitrary, for $i=0, j$ limit use clause $(D)$, for $i=0, j=\xi+1$ use clause $(E)$. For $i$ limit use clause $(C)$ (and check). For $i=\zeta+1$, if $j=0$ use clause $(F)$, if $j$ limit use clause $(D)$ and if $j=\xi+1$ use the existence of disjoint amalgamation (i.e. 5.11).

Lastly, choose $M_{3} \in K_{\lambda}$ which is $\left(\lambda, \operatorname{cf}\left(\delta_{3}^{a}\right)\right)$-saturated over $M_{\lambda \times \delta_{1}^{a}, \lambda \times \delta_{2}^{a}}$.

2) Similarly.

Proof of 8.7. Let $M_{i, j}, M_{3}$ be as in 8.8. For $x \in\{a, b\}$ as $N F_{\lambda, \bar{\delta}^{x}}\left(N_{0}^{x}, N_{1}^{x}, N_{2}^{x}, N_{3}^{x}\right)$, we know that there are witnesses $\left\langle N_{1, i}^{x}: i \leq \lambda \times \delta_{1}^{x}\right\rangle,\left\langle N_{2, i}^{x}: i \leq \lambda \times \delta_{1}^{x}\right\rangle$ for this, so $\left\langle N_{1, i}^{x}: i \leq \lambda \times \delta_{1}^{x}\right\rangle$ is $\leq_{\mathfrak{K}^{-}}$-increasing continuous and $\left(N_{1, i}^{x}, N_{1, i+1}^{x}\right) \cong\left(M^{*}, N^{*}\right)$.

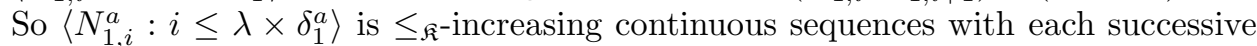
pair isomorphic to $\left(M^{*}, N^{*}\right)$ hence by $8.8(2)$ without loss of generality there is an isomorphism $g_{1}$ from $N_{1, \lambda \times \delta_{1}^{a}}^{a}$ onto $M_{\lambda \times \delta_{1}^{a}}$, mapping $N_{1, i}^{a}$ onto $M_{i, 0}$; remember $N_{1, \lambda \times \delta_{1}^{a}}^{a}=N_{1}^{a}$. Let $g_{0}=g_{1}\left\lceil N_{0}^{a}=g_{1}\left\lceil N_{1,0}^{a}\right.\right.$ so $g_{0} \circ f_{0}^{-1}$ is an isomorphism from $N_{0}^{b}$ onto $M_{0,0}$.

As $\delta_{1}^{b}=\delta_{2}^{a}$, using 8.8(2) fully without loss of generality there is an isomorphism $g_{2}$ from $N_{1, \lambda \times \delta_{2}^{a}}^{b}$ onto $M_{0, \lambda \times \delta_{2}^{a}}$ mapping $N_{1, j}^{b}$ onto $M_{0, j}$ (for $j \leq \lambda \times \delta_{2}^{a}$ ) and $g_{2}$ extends $g_{0} \circ f_{0}^{-1}$.

Now we want to use the weak uniqueness 8.6 and for this note:

( $\alpha) N F_{\lambda, \bar{\delta}^{a}}\left(N_{0}^{a}, N_{1}^{a}, N_{2}^{a}, N_{3}^{a}\right)$ as witnessed by $\left\langle N_{1, i}^{a}: i \leq \lambda \times \delta_{1}^{a}\right\rangle$, $\left\langle N_{2, i}^{a}: i \leq \lambda \times \delta_{1}\right\rangle$

[why? an assumption]

( $\beta) ~ N F_{\lambda, \bar{\delta}^{a}}\left(M_{0,0}, M_{\lambda \times \delta_{1}^{a}, 0}, M_{0, \lambda \times \delta_{2}^{a}}, M_{3}\right)$ as witnessed by the sequences $\left\langle M_{i, 0}: i \leq \lambda \times \delta_{1}\right\rangle,\left\langle M_{i, \lambda \times \delta_{2}^{a}}: i \leq \lambda \times \delta_{2}^{a}\right\rangle$

[why? check]

$(\gamma) g_{0}$ is an isomorphism from $N_{0}^{a}$ onto $M_{0,0}$

[why? see its choice]

( $\delta) g_{1}$ is an isomorphism from $N_{1}^{a}$ onto $M_{\lambda \times \delta_{1}^{a}, 0}$ mapping $N_{1, i}^{a}$ onto $M_{i, 0}$ for $i \leq \lambda \times \delta_{1}^{a}$ and extending $g_{0}$

[why? see the choice of $g_{1}$ and of $g_{0}$ ]

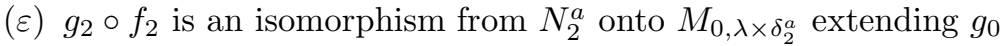

[why? $f_{2}$ is an isomorphism from $N_{2}^{a}$ onto $N_{1}^{b}$ and $g_{2}$ is an isomorphism from $N_{1}^{b}$ onto $M_{0, \lambda \times \delta_{1}^{a}}$ extending $g_{0} \circ f_{0}^{-1}$ and $\left.f_{0} \subseteq f_{2}\right]$. 
So there is by 8.6 an isomorphism $g_{3}^{a}$ from $N_{3}^{a}$ onto $M_{3}$ extending $g_{1}$ and $g_{2} \circ f_{2}$.

We next want to apply 8.6 to the $N_{1}^{b}$ 's; so note:

$(\alpha)^{\prime} N F_{\lambda, \bar{\delta}^{b}}\left(N_{0}^{b}, N_{1}^{b}, N_{2}^{b}, N_{3}^{b}\right)$ as witnessed by $\left\langle N_{1, i}^{b}: i \leq \lambda \times \delta_{2}^{a}\right\rangle$, $\left\langle N_{2, i}^{b}: i \leq \lambda \times \delta_{2}^{a}\right\rangle$

$(\beta)^{\prime} N F_{\lambda, \bar{\delta}^{b}}\left(M_{0,0}, M_{0, \lambda \times \delta_{2}^{a}}, M_{\lambda \times \delta_{1}^{a}, 0}, M_{3}\right)$ as witnessed by the sequences $\left\langle M_{0, j}: j \leq \lambda \times \delta_{2}^{a}\right\rangle,\left\langle M_{\lambda \times \delta_{1}^{a}, j}: j \leq \lambda \times \delta_{1}^{a}\right\rangle$

$(\gamma)^{\prime} g_{0} \circ\left(f_{0}\right)^{-1}$ is an isomorphism from $N_{0}^{b}$ onto $M_{0,0}$ [why? Check.]

$(\delta)^{\prime} g_{2}$ is an isomorphism from $N_{1}^{b}$ onto $M_{0, \lambda \times \delta_{2}^{a}}$ mapping $N_{1, j}^{b}$ onto $M_{0, j}$ for $j \leq \lambda \times \delta_{2}^{a}$ and extending $g_{0} \circ\left(f_{1}\right)^{-1}$

[why? see the choice of $g_{2}$ : it maps $N_{1, j}^{b}$ onto $M_{0, j}$ ]

$(\varepsilon)^{\prime} g_{1} \circ\left(f_{1}\right)^{-1}$ is an isomorphism from $N_{2}^{b}$ onto $M_{\lambda \times \delta_{0}^{a}}$ extending $g_{0}$ [why? remember $f_{1}$ is an isomorphism from $N_{1}^{a}$ onto $N_{2}^{b}$ extending $f_{0}$ and the choice of $g_{1}$ : it maps $N_{1}^{a}$ onto $\left.M_{\lambda \times \delta_{1,0}^{a}}\right]$.

So there is an isomorphism $g_{3}^{b}$ form $N_{3}^{b}$ onto $M_{3}$ extending $g_{2}, f_{1} \circ\left(f_{1}\right)^{-1}$. Lastly $\left(g_{3}^{b}\right)^{-1} \circ g_{3}^{a}$ is an isomorphism from $N_{3}^{a}$ onto $N_{3}^{b}$ (chase arrows). Also

$$
\begin{aligned}
\left(\left(g_{b}^{b}\right)^{-1} \circ g_{3}^{a}\right) \uparrow N_{1}^{a} & =\left(g_{3}^{b}\right)^{-1}\left(g_{3}^{a}\left\lceil N_{1}^{a}\right)\right. \\
& =\left(g_{3}^{b}\right)^{-1} g_{1}=\left(\left(g_{3}^{b}\right)^{-1}\left\lceil M_{\lambda \times \delta_{1,0}^{a}}\right) \circ g_{1}\right. \\
& =\left(g_{3}^{b}\left\lceil N_{2}^{b}\right)^{-1} \circ g_{1}=\left(\left(g_{1} \circ\left(f_{1}\right)^{-1}\right)^{-1}\right) \circ g_{1}\right. \\
& =\left(f_{1} \circ\left(g_{1}\right)^{-1}\right) \circ g_{1}=f_{1} .
\end{aligned}
$$

Similarly $\left(\left(g_{3}^{b}\right)^{-1} \circ g_{3}^{a}\right) \uparrow N_{2}^{a}=f_{2}$.

So we have finished.

8.9 Claim. [Uniqueness]. Assume for $x \in\{a, b\}$ we have $N F_{\lambda, \bar{\delta}^{x}}\left(N_{0}^{x}, N_{1}^{x}, N_{2}^{x}, N_{3}^{x}\right)$ and $c f\left(\delta_{1}^{a}\right)=c f\left(\delta_{1}^{b}\right), c f\left(\delta_{2}^{a}\right)=c f\left(\delta_{2}^{b}\right), c f\left(\delta_{3}^{a}\right)=c f\left(\delta_{3}^{b}\right)$, all $\delta_{\ell}^{x}$ limit ordinals.

If $f_{\ell}$ is an isomorphism from $N_{\ell}^{a}$ onto $N_{\ell}^{b}$ for $\ell<3$ and $f_{0} \subseteq f_{1}, f_{0} \subseteq f_{2} \underline{\text { then }}$ there is an isomorphism $f$ from $N_{3}^{a}$ onto $N_{3}^{b}$ extending $f_{1}, f_{2}$.

Proof. Let $\bar{\delta}^{c}=\left\langle\delta_{1}^{c}, \delta_{2}^{c}, \delta_{3}^{c}\right\rangle=\left\langle\delta_{2}^{a}, \delta_{1}^{a}, \delta_{3}^{a}\right\rangle$; by 8.4 there are $N_{\ell}^{c}$ (for $\ell \leq 3$ ) such that $N F_{\lambda, \bar{\delta}^{c}}\left(N_{0}^{c}, N_{1}^{c}, N_{2}^{c}, N_{3}^{c}\right)$. There is for $x \in\{a, b\}$ an isomorphism $g_{0}^{x}$ from $N_{0}^{a}$ onto $N_{0}^{c}$ (as $K_{\lambda}$ is categorical in $\lambda$ ) and without loss of generality $g_{0}^{b}=g_{0}^{a} \circ f_{0}$. Similarly for $x \in\{a, b\}$ there is an isomorphism $g_{1}^{x}$ from $N_{1}^{x}$ onto $N_{2}^{c}$ extending $g_{0}^{x}$ (as $N_{1}^{x}$ is $\left(\lambda, \operatorname{cf}\left(\delta_{1}^{x}\right)\right)$-saturated over $N_{0}^{x}$ and also $N_{2}^{c}$ is $\left(\lambda, \operatorname{cf}\left(\delta_{2}^{c}\right)\right)$-saturated over $N_{0}^{c}$ and $\left.\operatorname{cf}\left(\delta_{2}^{c}\right)=\operatorname{cf}\left(\delta_{1}^{a}\right)=\operatorname{cf}\left(\delta_{1}^{x}\right)\right)$ and without loss of genrality $\left.g_{1}^{b}=g_{1}^{a} \circ f_{1}\right)$. Similarly for $x \in\{a, b\}$ there is an isomorphism $g_{2}^{x}$ from $N_{2}^{x}$ onto $N_{1}^{c}$ extending $g_{0}^{x}$ (as $N_{2}^{x}$ is $\left(\lambda, \operatorname{cf}\left(\delta_{2}^{x}\right)\right)$-saturated over $N_{0}^{x}$ and also $N_{1}^{c}$ is $\left(\lambda, \operatorname{cf}\left(\delta_{1}^{c}\right)\right)$-saturated over $N_{0}^{c}$ and $\left.\operatorname{cf}\left(\delta_{1}^{c}\right)=\operatorname{cf}\left(\delta_{2}^{a}\right)=\operatorname{cf}\left(\delta_{2}^{x}\right)\right)$ and without loss of generality $g_{2}^{b}=g_{2}^{b} \circ f_{2}$.

So by 8.7 for $x \in\{a, b\}$ there is an isomorphism $g_{3}^{x}$ from $N_{3}^{x}$ onto $N_{3}^{c}$ extending $g_{1}^{x}$ 
and $g_{2}^{x}$. Now $\left(g_{3}^{b}\right)^{-1} \circ g_{3}^{a}$ is an isomorphism from $N_{3}^{a}$ onto $N_{3}^{b}$ extending $f_{1}, f_{2}$ as required.

8.10 Conclusion. [Symmetry].

If $N F_{\lambda,\left\langle\delta_{1}, \delta_{2}, \delta_{3}\right\rangle}\left(N_{0}, N_{1}, N_{2}, N_{3}\right)$ then $N F_{\lambda,\left\langle\delta_{2}, \delta_{1}, \delta_{3}\right\rangle}\left(N_{0}, N_{2}, N_{1}, N_{3}\right)$.

Proof. By 8.7 (and 8.9).

8.11 Claim. 1) In Definition 8.2 we can replace $\left(N_{1, i}, N_{1, i+1}\right) \cong\left(M^{*}, N^{*}\right)$ by $\left(N_{1, i}, N_{1, i+1}\right) \in K^{3, \text { uq }}$.

Proof. Like the proof of 8.7 (get $\left(M_{i, 0}, M_{i+1,0}\right) \cong\left(M^{*}, N^{*}\right)$, $\left.\left(M_{0, j}, M_{0, j+1}\right) \in K_{\lambda}^{3, \text { uq }}\right)$, but as we shall not use it, we do not elaborate. $\quad \square_{8.11}$

Now we turn to smooth amalgamation (not necessarily saturated, see Definition 8.3).

8.12 Claim. 1) If $N F_{\lambda, \bar{\delta}}\left(N_{0}, N_{1}, N_{2}, N_{3}\right)$ each $\delta_{\ell}$ limit then $N F_{\lambda}\left(N_{0}, N_{1}, N_{2}, N_{3}\right)$ (see Definition 8.3).

2) In Definition 8.3 we can add:

$(d)^{+} M_{\ell}$ is $(\lambda, c f(\lambda))$-saturated over $N_{0}$ and moreover over $N_{\ell}$,

(e) $M_{3}$ is $(\lambda, c f(\lambda))$-saturated over $M_{1} \cup M_{2}$ (actually this is given by $(f)(\zeta)$ of Definition 8.2).

Proof. 1) By 8.8 we can find $M_{i, j}$ for $i \leq \lambda \times\left(\delta_{1}+\lambda\right), j \leq \lambda \times\left(\delta_{2}+\lambda\right)$ for $\bar{\delta}^{\prime}=:\left\langle\delta_{1}+\lambda, \delta_{2}+\lambda, \delta_{3}\right\rangle$ and choose $M_{3}^{\prime} \in K_{\lambda}$ which is $\left(\lambda, \operatorname{cf}\left(\delta_{3}\right)\right)$-saturated over $M_{\lambda \times \delta_{1}, \lambda \times \delta_{2}}$. So $N F_{\lambda, \bar{\delta}}\left(M_{0,0}, M_{\lambda \times \delta_{1}, 0}, M_{0, \lambda \times \delta_{2}}, M_{3}\right)$, hence by 8.9 without loss of generality $M_{0,0}=N_{0}, M_{\lambda \times \delta_{1}, 0}=N_{1}, M_{0, \lambda \times \delta_{2}}=N_{2}$, and $N_{3}=M_{3}^{\prime}$. Lastly, let $M_{3}$ be $(\lambda, \operatorname{cf}(\lambda))$-saturated over $M_{3}^{\prime}$. Now clearly also

$N F_{\lambda,\left\langle\delta_{1}+\lambda, \delta_{2}+\lambda, \delta_{3}+\lambda\right\rangle}\left(M_{0,0}, M_{\lambda \times\left(\delta_{1}+\lambda\right), 0}, M_{0, \lambda \times\left(\delta_{2}+\lambda\right)}, M_{3}\right)$ and

$N_{0}=M_{0,0}, N_{1}=M_{\lambda \times \delta_{2}, 0} \leq_{\mathfrak{K}} M_{\lambda \times\left(\delta_{2}+\lambda\right), 0}, N_{2}=M_{0, \lambda \times \delta_{2}} \leq_{\mathfrak{K}} M_{0, \lambda \times\left(\delta_{2}+\lambda\right)}$

and $M_{\lambda \times\left(\delta_{1}+\lambda\right), 0}$ is $(\lambda, \operatorname{cf}(\lambda))$-saturated over $M_{\lambda \times \delta_{1}, 0}$ and $M_{0, \lambda \times\left(\delta_{2}+\lambda\right)}$ is

$(\lambda, \operatorname{cf}(\lambda))$-saturated over $M_{0, \lambda \times \delta_{2}}$ and $N_{3}=M_{3}^{\prime} \leq_{\mathfrak{K}} M_{3}$. So we get all the requirements for $N F_{\lambda}\left(N_{0}, N_{1}, N_{2}, N_{3}\right)$ (as witnessed by $\left.\left\langle M_{0,0}, M_{\lambda \times\left(\delta_{1}+\lambda\right), 0}, M_{0, \lambda \times\left(\delta_{2}+\lambda\right)}, M_{3}\right\rangle\right)$.

2) Similar proof.

8.13 Claim. [Uniqueness of smooth amalgamation]: If $N F_{\lambda}\left(N_{0}^{x}, N_{1}^{x}, N_{2}^{x}, N_{3}^{x}\right)$ for $x \in\{a, b\}, f_{\ell}$ an isomorphism from $N_{\ell}^{a}$ onto $N_{\ell}^{b}$ for $\ell<3$ and $f_{0} \subseteq f_{1}, f_{0} \subseteq f_{2}$ then $f_{1} \cup f_{2}$ can be extended to $a \leq_{\mathfrak{K}}$-embedding of $N_{3}^{a}$ into some $\leq_{\mathfrak{K}}$-extension of $N_{3}^{b}$ (so if $N_{3}^{x}$ is $(\lambda, \kappa)$-saturated over $N_{1}^{x} \cup N_{2}^{x}$ for $x=a, b$, we can extend $f_{1} \cup f_{2}$ to an isomorphism from $N_{3}^{a}$ onto $N_{3}^{b}$.

Proof. For $x \in\{a, b\}$ let the sequence $\left\langle M_{\ell}^{x}: \ell<4\right\rangle$ be a witness to $N F_{\lambda}\left(N_{0}^{x}, N_{1}^{x}, N_{2}^{x}, N_{3}^{x}\right)$ as in 8.3, 8.12(2) so in particular 
$N F_{\lambda,\langle\lambda, \lambda, \lambda\rangle}\left(M_{0}^{x}, M_{1}^{x}, M_{2}^{x}, M_{3}^{x}\right)$. By chasing arrows and uniqueness, i.e. 8.7 without loss of generality $M_{\ell}^{a}=M_{\ell}^{b}$ for $\ell<4$ and $f_{0}=\operatorname{id}_{N_{0}^{a}}$. As $M_{1}^{a}$ is $(\lambda, \operatorname{cf}(\lambda))$-saturated over $N_{1}^{a}$ and also over $N_{1}^{b}$ and $f_{1}$ is an isomorphism from $N_{1}^{a}$ onto $N_{1}^{b}$, clearly there is an automorphism $g_{1}$ of $M_{1}^{a}$ such that $f_{1} \subseteq g_{1}$, hence also id $N_{0}^{a}=f_{0} \subseteq$ $f_{1} \subseteq g_{1}$. Similarly there is an automorphism $g_{2}$ of $M_{1}^{a}$ extending $f_{1}$ hence $f_{0}$. So $g_{\ell} \in \operatorname{AUT}\left(M_{\ell}^{a}\right)$ for $\ell=1,2$ and $g_{1}\left\lceil M_{0}^{a}=f_{0}=g_{2} \uparrow M_{0}^{a}\right.$. By the uniqueness of $N F_{\lambda,\langle\lambda, \lambda, \lambda\rangle}$ (i.e. Claim 8.9) there is an automorphism $g_{4}$ of $M_{4}^{a}$ extending $g_{1} \cup g_{2}$. This proves the desired conclusion.

$\square_{8.13}$

8.14 Claim. Assume

(a) $\bar{\delta}=\left\langle\delta_{1}, \delta_{2}, \delta_{3}\right\rangle, \delta_{\ell}<\lambda^{+}$is a limit ordinal for $\ell=1,2,3$;

$N_{0} \leq_{\mathfrak{K}} N_{\ell} \leq_{\mathfrak{K}} N_{3}$ for $\ell=1,2$ and

(b) $N_{\ell}$ is $\left(\lambda, c f\left(\delta_{\ell}\right)\right)$-saturated over $N_{0}$

(c) $N_{3}$ is cf $\left(\delta_{3}\right)$-saturated over $N_{1} \cup N_{2}$.

Then $N F_{\lambda}\left(N_{0}, N_{1}, N_{2}, N_{3}\right)$ iff $N F_{\lambda, \bar{\delta}}\left(N_{0}, N_{1}, N_{2}, N_{3}\right)$.

Proof. The "if" direction holds by 8.13. For the "only if" direction, by the proof of 8.12(1) (and Definition 8.2, 8.3) we can find $M_{\ell}(\ell \leq 3)$ such that $N F_{\lambda, \bar{\delta}}\left(M_{0}, M_{1}, M_{2}, M_{3}\right)$ and clauses (b), (c), (d) of Definition 8.3 holds so by 8.12 also $N F_{\lambda}\left(M_{0}, M_{1}, M_{2}, M_{3}\right)$. Easily there are for $\ell<3$, an isomorphism $f_{\ell}$ from $M_{\ell}$ onto $N_{\ell}$ such that $f_{0}=f_{\ell} \uparrow$ $M_{\ell}$. By the uniqueness for smooth amalgamation (i.e. 8.13) we can find an isomorphism $f_{3}$ from $M_{3}$ onto $N_{3}$ extending $f_{1} \cup f_{2}$. So as $N F_{\lambda, \bar{\delta}}\left(M_{0}, M_{1}, M_{2}, M_{3}\right)$ holds also $N F_{\lambda, \bar{\delta}},\left(f_{0}\left(M_{0}\right), f_{3}\left(M_{1}\right), f_{3}\left(M_{2}\right), f_{3}\left(M_{3}\right)\right)$;

i.e. $N F_{\lambda, \bar{\delta}}\left(N_{0}, N_{1}, N_{2}, N_{3}\right)$ as required.

8.15 Claim. [Monotonicity]: If $N F_{\lambda}\left(N_{0}, N_{1}, N_{2}, N_{3}\right)$ and $N_{0} \leq_{\mathfrak{K}} N_{1}^{\prime} \leq_{\mathfrak{K}} N_{1}$ and $N_{0} \leq_{\mathfrak{K}} N_{2}^{\prime} \leq_{\mathfrak{K}} N_{2}$ and $N_{1}^{\prime} \cup N_{2}^{\prime} \subseteq N_{3}^{\prime} \leq_{\mathfrak{K}} N_{3}$ then $N F_{\lambda}\left(N_{0}, N_{1}^{\prime}, N_{2}^{\prime}, N_{3}^{\prime}\right)$.

Proof. Read Definition 8.3.

8.16 Claim. [Symmetry]: $N F_{\lambda}\left(N_{0}, N_{1}, N_{2}, N_{3}\right)$ holds if and only if $N F_{\lambda}\left(N_{0}, N_{2}, N_{1}, N_{3}\right)$ holds.

Proof. By Claim 8.10 (and Definition 8.3).

8.17 Claim. Assume $\alpha<\lambda^{+}$is an ordinal and for $x \in\{a, b, c\}$ the sequence $\left\langle N_{i}^{x}: i \leq \alpha\right\rangle$ is a $\leq_{\mathfrak{K}}$-increasing sequence of members of $K_{\lambda}$, for $x=a, b$ the sequence is $\leq_{\mathfrak{K}}$-increasing continuous for $i \leq \alpha, N_{i}^{b} \cap N_{\alpha}^{a}=N_{i}^{a}, N_{i}^{c} \cap N_{\alpha}^{a}=N_{i}^{a}, N_{i}^{a} \leq_{\mathfrak{K}}$ $N_{i}^{b} \leq_{\mathfrak{K}} N_{i}^{c}$ and $N_{i}^{c}$ is $\left(\lambda, \kappa_{i}\right)$-saturated over $N_{i}^{b}$ and $N F_{\lambda, \bar{\delta}^{i}}\left(N_{i}^{a}, N_{i+1}^{a}, N_{i}^{c}, N_{i+1}^{b}\right)$ (so $\left.i<\alpha \Rightarrow N_{i}^{c} \leq_{\mathfrak{K}} N_{i+1}^{b}\right)$ where 
$\bar{\delta}^{i}=\left\langle\delta_{1}^{i}, \delta_{2}^{i}, \delta_{3}^{i}\right\rangle$ sequence of limit ordinals, $i<\alpha \Rightarrow \delta_{2}^{i+1}=\delta_{i}^{3}$, and for $i<0$ limit, $c f\left(\delta_{i}^{3}\right)=\sum_{j<i} \delta_{j}^{3}, \delta_{1}=\sum_{\beta<\alpha} \delta_{\beta}^{1}$ and $\delta_{3}=\kappa_{\alpha}, \bar{\delta}=\left\langle\delta_{1}, \delta_{2}^{0}, \delta_{3}\right\rangle . \underline{\text { Then }} N F_{\lambda, \bar{\delta}}\left(N_{0}^{a}, N_{\alpha}^{a}, N_{0}^{b}, N_{\alpha}^{c}\right)$.

Proof. Use uniqueness of 8.9 , lastly use 8.9 to show $N_{\alpha}^{b}$ is $(\lambda, \operatorname{cf}(\alpha))$-saturated over $N_{\alpha}^{a} \cup N_{0}^{b}$.

8.18 Claim. Assume that $\alpha<\lambda^{+}$and for $x \in\{a, b\}$ we have $\left\langle N_{i}^{x}: i \leq \alpha\right\rangle$ is $\leq_{\mathfrak{K}}$-increasing continuously sequence of members of $K_{\lambda}$.

1) If $N F_{\lambda}\left(N_{i}^{a}, N_{i+1}^{a}, N_{i}^{b}, N_{i+1}^{b}\right)$ for each $i<\alpha \underline{\text { then }} N F_{\lambda}\left(N_{0}^{a}, N_{\alpha}^{a}, N_{0}^{b}, N_{\alpha}^{b}\right)$.

2) If $\alpha_{1}<\lambda^{+}, \alpha_{2}<\lambda^{+}$and $M_{i, j}\left(i \leq \alpha_{1}, j \leq \alpha_{2}\right)$ are as in 8.8, and for each $i<\alpha_{1}, j<\alpha_{2}$ we have:

$$
\begin{gathered}
M_{i, j+1} \bigcup_{M_{i, j}}^{M_{i+1, j+1}} M_{i+1, j} \\
\text { then } M_{i, 0} \bigcup_{M_{0,0}}^{M_{\alpha_{1}, \alpha_{2}}} M_{0, j} \text { for } i \leq \alpha_{1}, j \leq \alpha_{2} .
\end{gathered}
$$

Proof. 1) We first prove special cases and use them to prove more general cases.

Case A: $N_{i+1}^{a}$ is $\left(\lambda, \delta_{i}^{1}\right)$-saturated over $N_{i}^{a}$ and $N_{i+1}^{b}$ is $\left(\lambda, \delta_{i}^{2}\right)$-saturated over $N_{i+1}^{a} \cup N_{i}^{b}$ for $i<\alpha$.

We can choose by induction on $i \leq \alpha, N_{i}^{c} \in K_{\lambda}$ such that

(a) $N_{0}^{b} \leq_{\mathfrak{K}} N_{0}^{c} \leq_{\mathfrak{K}} N_{i+1}^{b}, N_{0}^{c}$ is $\left(\lambda, \delta_{0}^{2}\right)$-saturated over $N_{0}^{b}$, and $N F_{\lambda,\left\langle\delta_{1}^{0}, \delta_{2}^{0}, \delta_{2}^{0}\right\rangle}\left(N_{0}^{a}, N_{1}^{a}, N_{0}^{c}, N_{1}^{b}\right)$

(b) $N_{\alpha}^{c} \in K_{\lambda}$ is $\left(\lambda, \delta_{3}^{\alpha}\right)$-saturated over $N_{\alpha}^{b}$.

(Possible by uniqueness; i.e. 8.13 and monotonicity, i.e. 8.15). Now we can use 8.17.

Case B: For each $i<\alpha$ we have: $N_{i+1}^{a}$ is $\left(\lambda, \kappa_{i}\right)$-saturated over $N_{i}^{a}$.

Let $\bar{\delta}^{i}=\left(\kappa_{i}, \lambda, \lambda\right)$.

We can find a $\leq_{\mathfrak{K}}$-increasing sequence $\left\langle M_{i}^{x}: i \leq \alpha\right\rangle$ for $x \in\{a, b, c\}$, continuous for $x=a, b$ such that $i<\alpha \Rightarrow M_{i}^{b} \leq_{\mathfrak{K}} M_{i}^{c} \leq_{\mathfrak{K}} M_{i+1}^{b}$ and $M_{\alpha}^{b} \leq_{\mathfrak{K}} M_{\alpha}^{c}$ and $N F_{\lambda, \bar{\delta}^{i}}\left(M_{i}^{a}, M_{i+1}^{a}, M_{i}^{c}, M_{i+1}^{b}\right)$ by choosing $M_{i}^{a}, M_{i}^{b}, M_{i}^{c}$ by induction on $i$. By Case A we know that $N F_{\lambda}\left(M_{0}^{a}, M_{\alpha}^{a}, M_{0}^{b}, M_{\alpha}^{c}\right)$ holds.

We can now choose an isomorphism $f_{0}^{a}$ from $N_{0}^{a}$ onto $M_{0}^{a}$ (exists as $K$ is categorical in $\lambda$ ) and then a $\leq_{\mathfrak{K}}$-embedding of $N_{0}^{b}$ into $M_{0}^{b}$ extending $f_{0}^{a}$. Next we choose by induction on $i \leq \alpha, f_{i}^{a}$ an isomorphism from $N_{i}^{a}$ onto $M_{i}^{a}$ such that: $j<i \Rightarrow f_{j}^{a} \subseteq f_{i}^{a}$, possible by "uniqueness of the $\left(\lambda, \kappa_{i}\right)$-saturated model over $M_{i}^{a}$ ". 
Now we choose by induction on $i \leq \alpha$, a $\leq_{\mathfrak{K}}$-embedding $f_{i}^{b}$ of $N_{i}^{b}$ into $M_{i}^{b}$ extending $f_{i}^{a}$ and $f_{j}^{b}$ for $j<i$. For $i=0$ we have done it, for $i$ limit use $\bigcup_{j<i} f_{j}^{b}$, lastly for $i$ a successor ordinal let $i=j+1$, now we have

$(*)_{2} \quad N F_{\lambda}\left(M_{i}^{a}, M_{i+1}^{a}, f_{i}^{b}\left(N_{i}^{b}\right), M_{i+1}^{b}\right)$ [why? because $N F_{\lambda, \bar{\delta}^{i}}\left(M_{i}^{a}, M_{i+1}^{a}, M_{i}^{c}, M_{i+1}^{b}\right)$ by the choice of the $M_{\zeta}^{x}$ 's hence by 8.14 we have $N F_{\lambda}\left(M_{i}^{a}, M_{i+1}^{a}, M_{i}^{c}, M_{i+1}^{b}\right)$ and as $M_{i}^{a} \leq_{\mathfrak{K}} f_{i}^{b}\left(N_{i}^{b}\right) \leq M_{i}^{b}, M_{i}^{c}$ by 8.15 we get $\left.(*)_{2}\right]$.

By $(*)_{2}$ and the uniqueness of smooth amalgamation 8.13 there is $f_{i}^{b}$ as required. So without loss of generality $f_{\alpha}^{b}$ is the identity, so we have $N_{0}^{a}=M_{0}^{a}, N_{\alpha}^{a}=$ $M_{\alpha}^{a}, N_{0}^{b} \leq_{\mathfrak{K}} M_{0}^{b}, N_{\alpha}^{b} \leq_{\mathfrak{K}} M_{\alpha}^{b}$; also as said above $N F_{\lambda}\left(M_{0}^{a}, M_{\alpha}^{a}, M_{0}^{b}, M_{\alpha}^{b}\right)$ holds so by monotonicity i.e. 8.15 we get $N F_{\lambda}\left(N_{0}^{a}, N_{\alpha}^{a}, N_{0}^{b}, N_{\alpha}^{b}\right)$ as required.

\section{Case C: General case.}

We can find $M_{i}^{\ell}$ for $\ell<3, i \leq \alpha$ such that:

(a) $M_{i}^{\ell} \in K_{\lambda}$

(b) for each $\ell<3, M_{i}^{\ell}$ is $\leq_{\mathfrak{K}^{-}}$-increasing in $i$

(c) $M_{i}^{0}=N_{i}^{a}$

(d) $M_{i+1}^{\ell+1}$ is $(\lambda, \lambda)$-saturated over $M_{i+1}^{\ell} \cup M_{i}^{\ell+1}$ for $\ell<2, i<\alpha$

(e) $N F_{\lambda}\left(M_{i}^{\ell}, M_{i+1}^{\ell}, M_{i}^{\ell+1}, M_{i}^{\ell+1}\right)$ for $\ell<2, i<\alpha$

(f) $M_{0}^{\ell+1}$ is $(\lambda, \lambda)$-saturated over $M_{0}^{\ell}$

(g) for $\ell<2$ and $i<\alpha$ limit we have

$$
M_{i}^{\ell+1} \text { is }(\lambda, \lambda) \text {-saturated over } \bigcup_{j<i} M_{j}^{\ell+1} \cup M_{i}^{\ell}
$$

(h) for $i<\alpha$ limit we have

$$
N F_{\lambda}\left(\bigcup_{j<i} M_{j}^{\prime}, M_{i}^{1}, \bigcup_{j<i} M_{j}^{2}, M_{i}^{2}\right) .
$$

[how? as in the proof of 8.8].

Now note:

$(*)_{4} \quad M_{i}^{\ell+1}$ is $(\lambda, \operatorname{cf}(\lambda \times(1+i)))$-saturated over $M_{i}^{\ell}$ [why? If $i=0$ by clause $(f)$, if $i$ a successor ordinal by clause $(d)$ and if $i$ is a limit ordinal then by clause $(\mathrm{g})]$

$(*)_{5}$ for $i<\alpha, N F_{\lambda}\left(M_{i}^{0}, M_{i+1}^{0}, M_{i}^{2}, M_{i+1}^{2}\right)$ [why? we use Case B for $\alpha=2$ with $M_{i}^{0}, M_{i+1}^{0}, M_{i}^{1}, M_{i+1}^{1}$, $M_{i}^{2}, M_{i+1}^{2}$ here standing for $N_{0}^{a}, N_{0}^{b}, N_{1}^{a}, N_{1}^{b}, N_{2}^{a}, N_{2}^{b}$ there].

Now we continue as in Case B (using $f_{i}^{a}=\operatorname{id}_{N_{i}^{a}}$ and defining by induction on $i$ a $\leq_{\mathfrak{K}^{-}}$-embedding $f_{i}^{b}$ of $N_{i}^{b}$ into $M_{i}^{c}$ ).

2) By part (1) for each $i$ the sequences $\left\langle M_{\beta, i}: \beta \leq \alpha_{1}\right\rangle,\left\langle M_{\beta, i+1}: \beta \leq \alpha_{1}\right\rangle$ we get 
$M_{\alpha_{1}} \bigcup_{M_{0, i}}^{M_{\alpha_{1}, i+1}} M_{0, i+1}$ hence by symmetry (i.e. 8.13) we have $M_{0, i+1} \bigcup_{M_{0, i}}^{M_{\alpha_{1}, i+1}} M_{\alpha_{1}, i}$.

Applying part (1) to the sequences $\left\langle M_{0, j}: j \leq \alpha_{2}\right\rangle,\left\langle M_{\alpha_{1}, j}: j \leq \alpha_{2}\right\rangle$ we get $M_{\alpha_{1}, \alpha_{2}}$

$M_{0, \alpha_{2}} \bigcup_{M_{0,0}}^{\alpha_{1}, \alpha_{2}} M_{\alpha_{1}, 0}$ hence by symmetry (i.e. 8.13) we have $M_{\alpha_{1}, 0} \bigcup_{M_{0,0}}^{\bigcup_{1}, \alpha_{2}} M_{0, \alpha_{2}}$; by monotonicity, i.e 8.15 (or restriction of the matrix) we get the desired conclusion. $\square_{8.18}$

8.19 Conclusion. Assume $\left\langle N_{i}^{\ell}: i \leq \alpha\right\rangle$ is $\leq_{\mathfrak{K}}$-increasing continuous for $\ell=0,1$ where $N_{i}^{\ell} \in K_{\lambda}$ and $N_{i+1}^{1}$ is $\left(\lambda, \kappa_{\ell}\right)$-saturated over $N_{i+1}^{0} \cup N_{i}^{1}$ and $\mathrm{NF}_{\lambda}\left(N_{i}^{0}, N_{i}^{1}, N_{i+1}^{0}, N_{i+1}^{1}\right)$.

Then $N_{\alpha}^{1}$ is $\left(\lambda, \operatorname{cf}\left(\sum_{i<\alpha} \kappa_{i}\right)\right.$ )-saturated over $N_{\alpha}^{0} \cup N_{0}^{1}$ (if $\alpha$ is a limit ordinal, " $N_{i+1}^{1}$ is universal over $N_{i+1}^{0} \cup N_{i}^{1}$ " suffice).

Proof. The case $\alpha$ not limit is trivial so assume $\alpha$ is a limit ordinal. We choose by induction on $i \leq_{x} \alpha$, a sequence $\left\langle M_{i, \varepsilon}^{\prime}: \varepsilon \leq \varepsilon(i)\right\rangle$ such that:

(a) $\left\langle M_{i, \varepsilon}: \varepsilon \leq \varepsilon(i)\right\rangle$ is (strictly) $<_{\mathfrak{K}}$-increasing continuous

(b) $N_{i}^{0} \leq_{\mathfrak{K}} M_{i, \varepsilon} \leq_{\mathfrak{K}} N_{i}^{1}$

(c) $N_{i}^{0}=M_{i, 0}$

(d) $\varepsilon(i)$ is (strictly) increasing continuous in $i$,

(e) $j<i \& \varepsilon \leq \varepsilon(j) \Rightarrow M_{i, \varepsilon} \cap N_{j}^{1}=M_{j, \varepsilon}$

(f) $\varepsilon(0)=1, M_{i, 1}=N_{0}^{1}$

(g) for $i>0, \lambda$ divide $\varepsilon(i)$

(h) $N_{\ell}^{i} \leq_{\mathfrak{K}} M_{i+1, \varepsilon(i)+1}$.

If we succeed, then $\varepsilon(\alpha)$ is divisible by $\lambda$ and $\left\langle M_{i, \varepsilon}: \varepsilon \leq \varepsilon(\alpha)\right\rangle$ is (strictly) $<_{\mathfrak{K}^{-}}$ increasing continuous, $M_{\alpha, 0}=N_{\alpha}^{0}$, and $M_{\alpha, \varepsilon(\alpha)} \leq_{\mathfrak{K}} N_{\alpha}^{1}$ but it includes $N_{i}^{1}$ for $i<\alpha$ hence (as $\alpha$ is a limit ordinal) it includes $\bigcup_{i<\alpha} N_{i}^{1}=N_{\alpha}^{1}$; and by 7.5 we conclude that $N_{\alpha}^{1}=M_{\alpha, \varepsilon(\alpha)}$ is $\left(\lambda, \operatorname{cf}(\alpha)\right.$ )-saturated over $M_{\alpha, 1}$ hence over $N_{\alpha}^{0} \cup N_{0}^{1}$ (both $\prec M_{\alpha, i}$ ).

For $i=0$, and $i$ limit there is not much to do. For $i$ successor we use 8.20 below.

8.20 Conclusion. 1) If $N F_{\lambda}\left(N_{0}, N_{1}, N_{2}, N_{3}\right)$ and $\left\langle M_{0, \varepsilon}: \varepsilon \leq \varepsilon(*)\right\rangle$ is $\leq_{\mathfrak{K}}$-increasing continuous, $N_{0} \leq_{\mathfrak{K}} M_{0, \varepsilon} \leq_{\mathfrak{K}} M_{2}$ then we can find $\left\langle M_{1, \varepsilon}: \varepsilon \leq \varepsilon(*)\right\rangle$ and $N_{3}^{\prime}$ such that:

(a) $N_{3} \leq_{\mathfrak{K}} N_{3}^{\prime} \in K_{\lambda}$

(b) $\left\langle M_{1, \varepsilon}: \varepsilon \leq \varepsilon(*)\right\rangle$ is $\leq_{\mathfrak{K}}$-increasing continuous

(c) $M_{1, \varepsilon} \cap N_{2}=M_{0, \varepsilon}$

(d) $N_{1} \leq_{\mathfrak{K}} M_{1, \varepsilon} \leq_{\mathfrak{K}} N_{3}^{\prime}$

(e) if $M_{0,0}=N_{0}$ then $M_{1,0}=N$. 
2) if $N_{3}$ is universal over $N_{1} \cup N_{2}$, then without loss of generality $N_{3}=N_{2}$.

Proof. 1) Straight by uniqueness.

2) Follows by (1).

$\square_{8.19}, \square_{8.20}$ 


\section{$\S 9$ Nice extensions In $K_{\lambda^{+}}$}

9.1 Hypothesis. Assume the hypothesis 8.1.

So by $\S 8$ we have reasonable control on smooth amalgamation in $K_{\lambda}$. We use this to define "nice" extensions in $K_{\lambda^{+}}$. This is treated again in $\S 10$.

9.2 Definition. 1) Let $M_{0} \leq_{\lambda^{+}}^{*} M_{1}$ mean:

(a) $M_{\ell} \in K_{\lambda^{+}}$, for $\ell=0,1$

(b) we can find $\bar{M}^{\ell}=\left\langle M_{i}^{\ell}: i<\lambda^{+}\right\rangle$, a representation of $M^{\ell}$, so $M_{i}^{\ell} \in K_{\lambda}$

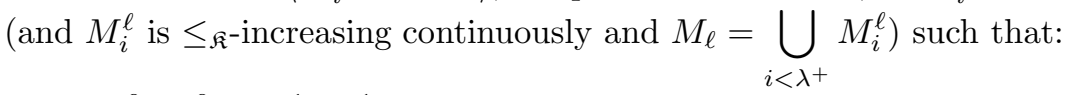
$N F_{\lambda}\left(M_{i}^{0}, M_{i+1}^{0}, M_{i}^{1}, M_{i+1}^{1}\right)$ for $i<\lambda^{+}$.

2) Let $M_{0}<_{\lambda^{+}, \kappa}^{+} M_{1}$ mean $M_{0} \leq_{\lambda^{+}}^{*} M_{1}$ by some witnesses $M_{i}^{\ell}\left(\right.$ for $\left.i<\lambda^{+}, \ell<2\right)$ such that $N F_{\lambda,\langle\kappa, 1, \kappa\rangle}\left(M_{i}^{0}, M_{i+1}^{0}, M_{i}^{1}, M_{i+1}^{1}\right)$. If $\kappa=\lambda$, we omit it.

9.3 Claim. 1) If $M_{0} \leq_{\lambda^{+}} M_{1}$ and $\bar{M}^{\ell}=\left\langle M_{i}^{\ell}: i<\lambda^{+}\right\rangle$is a representation of $M_{\ell}$ (as in 8.18) then for some club $E$ of $\lambda^{+}$, for every $\alpha<\beta$ from $E$ we have $N F_{\lambda}\left(M_{\alpha}^{0}, M_{\beta}^{0}, M_{\alpha}^{1}, M_{\beta}^{1}\right)$.

2) Similarly for $<_{\lambda^{+}, \kappa}^{+}$; if $M_{0}<_{\lambda^{+}, \kappa_{\ell}} M_{1}, \bar{M}^{\ell}=\left\langle\bar{M}_{i}^{\ell}: i<\lambda^{+}\right\rangle$a representation of $M_{\ell}$ for $\ell=1,2$ then for some club $E$ of $\lambda^{+}$for every $\alpha<\beta$ from $E$ we have $N F_{\lambda,\langle\operatorname{cf}(\alpha), 1, \operatorname{cf}(\alpha)\rangle}\left(M_{\alpha}^{0}, M_{\beta}^{0}, M_{\alpha}^{1}, M_{\beta}^{0}\right)$.

3) The $\kappa$ in Definition 9.2(2) does not matter. In fact, if $\left\langle M_{i}^{\ell}: i\left\langle\lambda^{+}\right\rangle\right.$are as in 9.2(1) then for some club $E$ of $\lambda^{+}$we have: $\alpha \in E \Rightarrow M_{\alpha}^{1} \cap M_{0}=M_{\alpha}^{0}$ and $\alpha<\beta \& \alpha \in E \& \beta \in E \Rightarrow\left[M_{\beta}^{1}\right.$ is $c f(\beta)$-saturated over $\left.M_{\beta}^{0} \cup M_{\alpha}^{1}\right]$.

Proof. 1) Straight by 8.18 .

2) Easy.

3) By 8.19. (Could have used 7.8).

9.4 Claim. 1) For every $\kappa=c f(\kappa) \leq \lambda$ and $M_{0} \in K_{\lambda^{+}}$for some $M_{1} \in K_{\lambda^{+}}$we have $M_{0}<_{\lambda^{+},{ }_{\kappa}}^{+} M_{1}$.

2) $<_{\lambda^{+}}^{*}$ are $<_{\lambda^{+}, \kappa}^{+}$are transitive.

3) If $M_{0} \leq_{\mathfrak{K}} M_{1} \leq_{\mathfrak{K}} M_{2}$ and $M_{0} \leq_{\lambda^{+}}^{*} M_{2}$ then $M_{0} \leq_{\lambda^{+}}^{*} M_{1}$.

4) [transitivity] If $M_{0} \leq_{\lambda^{+}}^{*} M_{1}<_{\lambda^{+}, \kappa}^{+} M_{2}$ then $M_{0}<_{\lambda^{+},{ }_{\kappa}}^{*} M_{2}$.

Proof. 1) Let $\left\langle M_{i}^{0}: i<\lambda^{+}\right\rangle$be a representation of $M_{0}$ such that $M_{i+1}^{0}$ is $(\lambda, \kappa)$-saturated over $M_{i}^{0}$. We choose by induction on $i, M_{i}^{1} \in K_{\lambda}$ such that $\left\langle M_{i}^{1}: i<\lambda^{+}\right\rangle$is $<_{\mathfrak{K}}$-increasing continuously, $M_{i}^{0} \leq_{\mathfrak{K}} M_{i}^{1}, M_{i}^{1} \cap M_{0}=M_{i}^{0}$ and $N F_{\lambda,\langle\kappa, 1, \kappa\rangle}\left(M_{i}^{0}, M_{i+1}^{0}, M_{i}^{1}, M_{i+1}^{1}\right)$. We can do it by 7.7(4).

2) Concerning $<_{\lambda^{+}, \kappa}^{+}$use 9.3 and 8.18 (i.e. transitivity for smooth amalgamations). Now the proof for $<_{\lambda^{+}}^{*}$ is similar.

3) By monotonicity for smooth amalgamations; i.e. 8.15.

4) Check. 
9.5 Claim. 1) If $M_{0} \leq_{\lambda^{+}}^{*} M_{\ell}$ for $\ell=1,2, \kappa=c f(\kappa) \leq \lambda$ and $a \in M_{2} \backslash M_{0} \underline{\text { then }}$ for some $M_{3}$ and $f$ we have: $M_{1}<_{\lambda^{+},{ }_{\kappa}}^{+} M_{3}$ and $f$ is an $\leq_{\mathfrak{K}}$-embedding of $M_{2}$ into $M_{3}$ over $M_{0}$ with $f(a) \notin M_{1}$, moreover, $f\left(M_{2}\right) \leq_{\lambda^{+}}^{*} M_{3}$.

2) [uniqueness] Assume $M_{0}<_{\lambda^{+},{ }_{\kappa}}^{+} M_{\ell}$ for $\ell=1,2$ then there is an isomorphism $f$ from $M_{1}$ onto $M_{2}$ over $M_{0}$.

Proof. We first prove part (2).

2) By 9.3(1) + (2) there are representations $\bar{M}^{\ell}=\left\langle M_{i}^{\ell}: i<\lambda^{+}\right\rangle$of $M_{\ell}$ for $\ell<3$ such that: $M_{i}^{\ell} \cap M_{0}=M_{0}^{\ell}$ and $N F_{\lambda,\langle\kappa, \mathrm{cf}(\kappa \times i), \kappa\rangle}\left(M_{i}^{0}, M_{i+1}^{0}, M_{i}^{\ell}, M_{i+1}^{\ell}\right)$.

Now we choose by induction on $i<\lambda$ an isomorphism $f_{i}$ from $M_{i}^{1}$ onto $M_{i}^{2}$, increasing with $i$ and being the identity over $M_{i}^{0}$. For $i=0$ use " $M_{0}^{\ell}$ is $(\lambda, \kappa)$ saturated over $M_{0}^{0}$ for $\ell=1,2$ " which holds by 8.2. For $i$ limit take unions, for $i$ successor ordinal use uniqueness Claim 8.9.

Proof of part (1). Let $\kappa=\aleph_{0}$, by 9.4(1) there are for $\ell=1,2$ models $N_{\ell}^{*} \in K_{\lambda^{+}}$ such that $M_{\ell}<_{\lambda^{+}, \kappa}^{+} N_{\ell}^{*}$. Now let $\bar{M}^{\ell}=\left\langle M_{i}^{\ell}: i<\lambda^{+}\right\rangle$be a representation of $M_{\ell}$ for $\ell=0,1,2$ and let $\bar{N}^{\ell}=\left\langle N_{i}^{\ell}: i<\lambda^{+}\right\rangle$be a representation of $N_{\ell}^{*}$ for $\ell=1,2$. By 9.4(4) and 9.3(3) without loss of generality $N F_{\lambda}\left(M_{i}^{0}, M_{i+1}^{0}, M_{i}^{\ell}, M_{i+1}^{\ell}\right)$ for $\ell=1,2$ and $N F_{\lambda,\langle\kappa, 1, \kappa\rangle}\left(M_{i}^{\ell}, M_{i+1}^{\ell}, N_{i}^{\ell}, N_{i+1}^{\ell}\right)$ respectively. Now clearly $N_{0}^{\ell}$ is $(\lambda, \kappa)$-saturated over $M_{0}^{\ell}$ hence over $M_{0}^{0}$ (for $\left.\ell=1,2\right)$ so there is an isomorphism $f_{0}$ from $N_{0}^{2}$ onto $N_{0}^{1}$ extending $\operatorname{id}_{M_{0}^{0}}$ and $f(a) \notin M_{0}^{1}$.

We continue as in the proof of part (2). In the end $f=\bigcup_{i<\lambda^{+}} f_{i}$ is an isomorphism of $N_{2}$ onto $N_{1}$ over $M_{0}$ and as $f_{0}^{1}(a)$ is well defined and in $N_{0}^{1} \backslash M_{0}^{1}$ clearly $f(a)=f_{0}(a) \notin M_{1}$, as required.

9.6 Claim. If $\delta$ is a limit ordinal $<\lambda^{+2}$ and $\left\langle M_{i}: i<\delta\right\rangle$ is a $\leq_{\lambda^{+}}^{*}$-increasing continuous then $M_{i} \leq_{\lambda^{+}}^{*} \bigcup_{j<\delta} M_{j}$ for each $i<\delta$.

Proof. We prove it by induction on $\delta$. Now if $C$ is a club of $\delta$ with $i \in C$, then we can replace $\left\langle M_{j}: j<\delta\right\rangle$ by $\left\langle M_{j}: j \in C\right\rangle$ so without loss of generality $\delta=\operatorname{cf}(\delta)$, so $\delta \leq \lambda^{+}$; clearly it is enough to prove $M_{0} \leq_{\lambda^{+}}^{*} \bigcup_{j<\delta} M_{j}$. Let $\left\langle M_{\zeta}^{i}: \zeta<\lambda^{+}\right\rangle$be a representation of $M_{i}$.

Case A: $\delta<\lambda^{+}$.

Without loss of generality (see 9.3(1)) for every $i<j<\delta$ and $\zeta<\lambda^{+}$we have: $M_{\zeta}^{j} \cap M_{i}=M_{\zeta}^{i}$ and $N F_{\lambda}\left(M_{\zeta}^{i}, M_{\zeta+1}^{i}, M_{\zeta}^{j}, M_{\zeta+1}^{j}\right)$. Let $M_{\zeta}^{\delta}=\bigcup_{i<\delta} M_{\zeta}^{i}$, so $\left\langle M_{\zeta}^{\delta}: \zeta\left\langle\lambda^{+}\right\rangle\right.$is $\leq_{\mathfrak{K}^{-}}$-increasingly continuous sequence of members of $K_{\lambda}$ with limit $M_{\delta}$, and for $i<\delta, M_{\zeta}^{\delta} \cap M_{i}=M_{\zeta}^{i}$. By symmetry (see 8.16) we have $N F_{\lambda}\left(M_{\zeta}^{i}, M_{\zeta}^{i+1}, M_{\zeta+1}^{i}, M_{\zeta+1}^{i+1}\right)$ so as $\left\langle M_{\zeta}^{i}: i \leq \delta\right\rangle,\left\langle M_{\zeta+1}^{i}: i \leq \delta\right\rangle$ are $\leq_{\mathfrak{K}}$-increasingly continuous by 8.18 we know $N F_{\lambda}\left(M_{\zeta}^{0}, M_{\zeta}^{\delta}, M_{\zeta+1}^{0}, M_{\zeta+1}^{\delta}\right)$ hence by symmetry (8.16) 
we have $N F_{\lambda}\left(M_{\zeta}^{0}, M_{\zeta+1}^{0}, M_{\zeta}^{\delta}, M_{\zeta+1}^{\delta}\right)$.

So $\left\langle M_{\zeta}^{0}: \zeta<\lambda^{+}\right\rangle,\left\langle M_{\zeta}^{\delta}: \zeta<\lambda^{+}\right\rangle$are witnesses to $M_{0} \leq_{\lambda^{+}}^{*} M_{\delta}$.

Case B: $\delta=\lambda^{+}$.

By 9.3(1) (using normality of the club filter, restricting to a club of $\lambda^{+}$and renaming), without loss of generality for $i<j \leq 1+\zeta<1+\xi<\lambda^{+}$we have $M_{\zeta}^{j} \cap M_{i}=M_{\zeta}^{i}$, and $N F_{\lambda}\left(M_{\zeta}^{i}, M_{\xi}^{i}, M_{\zeta}^{j}, M_{\xi}^{j}\right)$. Let us define $M_{i}^{\lambda^{+}}=\bigcup_{j<1+i} M_{i}^{j}$. So $\left\langle M_{i}^{\lambda^{+}}: i<\lambda^{+}\right\rangle$is a representation of $M_{\lambda^{+}}^{\lambda}=M_{\delta}$ and continue as before. $\quad \square_{9.6}$

9.7 Claim. Assume $M_{0}<_{\lambda^{+}, \kappa}^{+} M_{2}$ and $a \in M_{2} \backslash M_{0}$, and for some $N \leq_{\mathfrak{K}} M_{0}$ we have: $N \in K_{\lambda}$ and $\operatorname{tp}\left(a, N, M_{2}\right)$ is minimal. Then we can find $M_{1}$, $\bar{M}^{0}=\left\langle M_{0, i}: i<\lambda^{+}\right\rangle, \bar{M}^{1}=\left\langle M_{1, i}: i<\lambda^{+}\right\rangle$such that:

(a) $\bar{M}^{0}$ is a $\leq_{\mathfrak{K}}$-representation of $M_{0}$

(b) $\bar{M}^{1}$ is a representation of $M_{1}\left(\in K_{\lambda^{+}}\right), a \in M_{1, i}$, for all $i$

(c) $M_{0} \leq_{\mathfrak{K}} M_{1} \leq_{\mathfrak{K}} M_{2}$

(d) for $i<\lambda^{+}$we have $N F_{\lambda,\langle\lambda, 1,1\rangle}\left(M_{0, i}, M_{0, i+1}, M_{1, i}, M_{1, i+1}\right)$ (hence $M_{\ell, i}=M_{\ell+1, i} \cap M_{\ell}$ )

(e) $\left(M_{0, i}, M_{1, i}, a\right) \in K_{\lambda}^{3}$ is minimal and reduced.

Proof. Let $\left\langle M_{0, i}: i\left\langle\lambda^{+}\right\rangle,\left\langle M_{2, i}: i<\lambda^{+}\right\rangle\right.$be representations of $M_{0}, M_{2}$ respectively, as required in 9.2(2) and without loss of generality $N \leq_{\mathfrak{K}} M_{0,0}$ and $a \in M_{2,0}$. We now choose by induction on $\zeta<\lambda^{+}$, an ordinal $i(\zeta)$ and models $M_{1, i(\zeta)}, M_{3, i(\zeta)}$ such that:

(A) $i(\zeta)<\lambda^{+}$is increasing continuous in $\zeta$ and $a \in M_{2, i(0)} \backslash M_{0, i(0)}, N \leq_{\mathfrak{K}} M_{0, i(0)}$

(B) $M_{0, i(\zeta)} \leq_{\mathfrak{K}} M_{1, i(\zeta)} \leq_{\mathfrak{K}} M_{3, i(\zeta)}$ and $M_{2, i(\zeta)} \leq_{\mathfrak{K}} M_{3, i(\zeta)}$

(C) $a \in M_{1, i(0)}$ and $\left(M_{0, i(\zeta)}, M_{1, i(\zeta)}, a\right)$ is minimal and reduced

$(D)$ for $\xi<\zeta$ and $(\ell, m) \in\{(0,1),(0,2),(1,3),(2,3)\}$ we have $N F_{\lambda}\left(M_{\ell, i(\xi)}, M_{\ell, i(\zeta)}, M_{m, i(\xi)}, M_{m, i(\zeta)}\right)$

(E) $M_{1, i(\zeta)}, M_{3, i(\zeta)}$ is $\leq_{\mathfrak{K}}$-increasing continuous in $\zeta$.

For $\zeta=0$ note that for $i(0)<\lambda^{+}, a \in M_{2, i(0)}$ and $M_{2, i(0)}$ is universal over $M_{0, i(0)}$.

For $\zeta$ limit let $i(\zeta)=\bigcup_{\xi<\zeta} i(\xi)$ and $M_{1, i(\zeta)}=\bigcup_{\xi<\zeta} M_{1, i(\zeta)}$.

For $\zeta=\xi+1$, there is $i(\zeta) \in\left(i(\xi), \lambda^{+}\right)$and a model $N_{\zeta}$ such that $M_{1, i(\xi)} \leq_{\mathfrak{K}}$

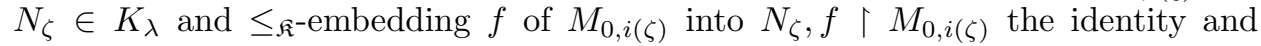
$\left(f\left(M_{0, i(\xi)}\right), N_{\zeta}, a\right)$ is minimal and reduced. By uniqueness (i.e. Claim 8.2) we can find such $N$ satisfying $(\exists M)\left(N \leq_{\mathfrak{K}} M \in K_{\lambda} \& M_{1, i(\zeta)} \leq_{\mathfrak{K}} M\right)$. So we can carry the induction.

Lastly, by uniqueness of $<_{\lambda, \kappa}^{+}$we can make $M_{3}=\bigcup_{\zeta<\lambda^{+}} M_{3, i(\zeta)}$ to be $\leq_{\mathfrak{K}} M_{2}$ as required. 
9.8 Definition. If $\left(M_{0}, M_{1}, a\right)$ are as in 9.7 (a)-(e) we say $\left(M_{0}, M_{1}, a\right)$ is $\lambda^{+}$-locally reduced nice and minimal ( $\lambda^{+}$-l.r.n.m.). We omit "nice" if we omit clause $(d)$.

9.9 Claim. If $\left(M_{0}, M_{1}, a\right)$ is $\lambda^{+}$-l.r.n.m. then $\left(M_{0}, M_{1}, a\right) \in K_{\lambda^{+}}^{3}$ is reduced.

Proof. Check.

Using also 7.5 :

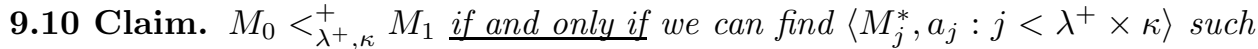
that:

(a) $M_{j}^{*}$ is $\leq_{\mathfrak{K}^{-}}$increasing continuous (in $K_{\lambda^{+}}$)

(b) $\left(M_{j}^{*}, M_{j+1}^{*}, a_{j}\right)$ is $\lambda^{+}$-l.r.n.m.

(c) $M_{0}^{*}=M_{0}, M_{j}^{*} \cup M_{j}^{*}=M_{1}$

(d) for some $N \leq M_{0}, N \in K_{\lambda}$ and minimal reduced $p \in S(N)$, for every $j$.

Proof. We can find $\left\langle M_{j}^{*}: j \leq \lambda^{+} \times \kappa\right)$ satisfying clauses $(a),(b)$ and $(d)$. By 5.6 easily $M_{0}^{*}<_{\lambda}^{+} M_{\lambda^{+}}^{*}$. Now by the uniqueness $(=9.5(2))+$ categoricity of $K$ in $\lambda^{+}$, we are done.

9.11 Claim. In $\left(K_{\lambda^{+}},<_{\lambda^{+}}^{*}\right)$ we have disjoint amalgamation.

Proof. First redo 9.5 assuming $\left(M_{0}, M_{\ell}, a_{\ell}\right)$ for $\ell=1,2$ is $\lambda^{+}$-l.r.n.m., and getting $a_{1} \notin f\left(M_{2}\right), f\left(a_{2}\right) \notin M_{1}$ (and we can start with this). By 9.9 we get $M_{1} \cap f\left(M_{2}\right)=M_{3}$, so we have disjoint amalgamation. By 9.10 and chasing arrows we get it in general.

$\square 9.11$

Remark. This is like the proof of disjoint amalgamations in 5.11. 


\section{$\S 10$ NON-STRUCTURE FOR $\leq_{\lambda^{+}}^{*}$}

10.1 Hypothesis. Assume the hypothesis 8.1 and the further model theoretic properties deduced since then (including 6.7 but not 6.12 .)

It would have been nice to prove all disjoint amalgamations in $K_{\lambda}$ are $N F_{\lambda}$, but this is, at this point, not clear. But as we look upward (i.e. want to prove statement on $K_{>\lambda^{+}}$) and $<_{\lambda^{+}}^{*}$ is very nice, it will be essentially just as well if for $M, N \in K_{\lambda^{+}}$ we have $M \leq_{\mathfrak{K}} N \Rightarrow M \leq_{\lambda^{+}}^{*} N$. Our intention is to assume $M^{*} \leq_{\mathfrak{K}} N^{*}$ is a counterexample of this statement and we would like to say that in a sense this implies the existence of many types over $M^{*}$ so that we can construct many models in $\lambda^{+2}$. Note: building models in $K_{\lambda^{+}}, K_{\lambda^{++}}$by approximations in $K_{\lambda}$ is nice if we use the smooth amalgamation but we do not have it for non-smooth ones. So we shall use $M^{*} \in K_{\lambda^{+}}$being saturated so it has many automorphisms.

10.2 Claim. 1) Assume $M_{1} \leq_{\mathfrak{K}} M_{2}$ are in $K_{\lambda^{+}}$. Then we can find $M_{0} \in K_{\lambda^{+}}$ such that $M_{0}<_{\lambda, \kappa}^{+} M_{1}$ and $M_{0} \leq_{\lambda^{+}}^{*} M_{2}$.

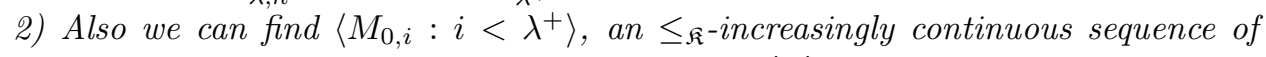
members of $K_{\lambda^{+}}$such that $M_{0, i}<_{\lambda^{+}}^{*} M_{0, i+1}$ and $\bigcup_{i<\lambda^{+}} M_{0, i}=M_{1}$ and $i<\lambda^{+} \Rightarrow M_{0, i} \leq_{\lambda^{+}}^{*} M_{2}$.

Proof. Let $\left(M^{*}, N^{*}\right) \in K_{\lambda}^{3 \text {,uq }}$ be from 8.1(2). Let $\left\langle M_{\ell, i}: i\left\langle\lambda^{+}\right\rangle\right.$be a representation of $M_{\ell}$ for $\ell=1,2$ and without loss of generality $M_{\ell, i+1}$ is $(\lambda, \lambda)$-saturated over $M_{\ell, i}$ for $\ell=1,2$ and $M_{2, i} \cap M_{1}=M_{1, i}$.

1) Now choose by induction on $i, M_{0, i}$ such that:

(a) $M_{0, i} \leq_{\mathfrak{K}} M_{1, i}$

(b) $M_{0, i}$ is $\leq_{\mathfrak{K}}$-increasing continuous

(c) $M_{0, i+1} \cap M_{1, i}=M_{0, i}$

(d) $M_{1, i+1}$ is $(\lambda, \operatorname{cf}(\lambda))$-saturated over $M_{1, i} \cup M_{0, i+1}$

(e) $\left(M_{0, i}, M_{0, i+1}\right) \cong\left(M^{*}, N^{*}\right)$.

There is no problem to carry the definition. Now let $M_{0}=\bigcup_{i<\lambda^{+}} M_{0, i}$ so $M_{0}<_{\lambda^{+}}^{+} M_{1}$ and $M_{0} \leq_{\lambda^{+}}^{*} M_{2}$ are checked by their definitions.

2) We choose by induction on $i<\lambda^{+},\left\langle M_{\varepsilon, i}^{*}: \varepsilon \leq 1+i\right\rangle$ such that:

(a) $M_{1+i, i}^{*}=M_{1, \lambda \times(1+\varepsilon) \times i}$

(b) For each $\varepsilon$ the sequence $\left\langle M_{\varepsilon, j}^{*}: \varepsilon \leq j \leq i\right\rangle$ is $\leq_{\mathfrak{R}^{-}}$-increasing continuous

(c) For each $i$, the sequence $\left\langle M_{\varepsilon, i}^{*}: \varepsilon \leq 1+i\right\rangle$ is $\leq_{\mathfrak{K}^{-}}$-increasing continuous

(d) $M_{\varepsilon, i}^{*} \cap M_{\zeta, j}^{*}=M_{\min \{\varepsilon, \zeta\}, \min \{i, j\}}^{*}$

(e) $M_{\varepsilon+1, i+1}^{*}$ is $(\lambda, \operatorname{cf}(\lambda \times(1+\varepsilon)))$-saturated over $M_{\varepsilon, i+1}^{*} \cup M_{\varepsilon+1, i}^{*}$

(f) $N F_{\lambda,\langle\lambda, 1, \lambda\rangle}\left(M_{\varepsilon, i}^{*}, M_{\varepsilon+1, i}^{*}, M_{\varepsilon, i+1}^{*}, M_{\varepsilon+1, i+1}^{*}\right)$

(g) for $\varepsilon<1+i$ we have $N F_{\lambda}\left(M_{\varepsilon, i}^{*}, M_{\varepsilon, i+1}^{*}, M_{2, \lambda \times \lambda \times i}, M_{2, \lambda \times \lambda \times(i+1)}\right)$. 
For $i=0, i$ limit no problem, for $i=j+1$ first choose $N_{i, \varepsilon, \zeta} \leq_{\mathfrak{K}} M_{1, \lambda \times \lambda \times i+\lambda \times \zeta}$ for $\zeta \leq \lambda \times(1+i), \leq_{\mathfrak{K}}$-increasing continuous, $N_{i, \varepsilon, \zeta} \in K_{\lambda}, N_{i, \varepsilon, 0}=M_{1, j}\left(=M_{1+i, i}^{*}\right)$, $\left(N_{i, \varepsilon, \zeta}, N_{i, \varepsilon, \zeta+1}\right) \cong\left(M^{*}, N^{*}\right)$ and $N_{i, \varepsilon, \zeta+1} \cap M_{2, \lambda \times \lambda \times i+\lambda \times \zeta}=N_{i, \varepsilon, \zeta}$.

Now by 5.6 , without loss of generality $N_{i, \varepsilon, \lambda}$ is $(\lambda, \lambda)$-saturated over $M_{1, j}$, and we choose it as $M_{1+j, i}^{*}$, and we choose $M_{1, i+1}^{*}$ as $M_{1+i, i}$ note that clauses (a) and (f) holds. Now we can find $N_{\varepsilon, j_{1}}$ for $j_{1}<1+j$ as in 8.8 and use uniqueness of the $(\lambda, \lambda \times(1+i))$-saturated model over $M_{1, j}$.

10.3 Conclusion. Assume $M \leq_{\mathfrak{K}} N$ are from $K_{\lambda^{+}}$. If $\left\langle M_{i}: i<\lambda^{+}\right\rangle$is $\leq_{\lambda^{+}}^{*}$-increasing continuous and for each $i$ for some $N_{i}$ we have $M_{i}<_{\lambda^{+}}^{+} N_{i} \leq_{\lambda^{+}}^{*} M_{i+1}$ then for some $\left(M^{\prime}, N^{\prime}\right)$ we have:

$$
\begin{gathered}
\left(M^{\prime}, N^{\prime}\right) \cong(M, N) \\
M^{\prime}=\bigcup_{i<\lambda} M_{i} \\
i<\lambda^{+} \Rightarrow M_{i} \leq_{\lambda^{+}}^{*} N^{\prime} .
\end{gathered}
$$

Proof. By 10.2(2) and as we know $M^{a} \leq_{\lambda^{+}}^{*} M^{b} \leq_{\lambda^{+}}^{+} M^{c} \Rightarrow M^{a}<_{\lambda^{+}}^{+} M^{c}$ and the uniqueness of $M^{\prime \prime}$ over $M^{\prime}$ when $M^{\prime}<_{\lambda^{+}}^{+} M^{\prime \prime}$.

10.4 Lemma. If $\leq_{\mathfrak{K}} \mid K_{\lambda^{+}}$is not $\leq_{\lambda^{+}}^{*} \underline{\text { then }} I\left(\lambda^{+2}, K\right)=2^{\lambda^{+2}}$.

Proof. Let $S \subseteq\left\{\delta<\lambda^{+2}: \operatorname{cf}(\delta)=\lambda^{+}\right\}$be stationary. We shall below construct a model $M_{S} \in \bar{K}_{\lambda^{+2}}$ such that from the isomorphism type of $M_{1}^{S}$ we can reconstruct $S / \mathscr{D}_{\lambda^{+2}}$; this clearly suffices. Choose $M \leq_{\mathfrak{K}} N$ from $K_{\lambda^{+}}$such that $\neg\left(M \leq_{\lambda^{+}}^{*} N\right)$ so by $9.4(3)$ without loss of generality $|N \backslash M|=\lambda^{+}$.

We choose by induction on $\alpha<\lambda^{+2}$ a model $M_{\alpha}^{S}$ such that:

(a) $M_{\alpha}^{S} \in K_{\lambda^{+}}$has universe $\lambda \times(1+\alpha)$

(b) for $\beta<\alpha$ we have $M_{\beta}^{S} \leq_{\mathfrak{K}} M_{\alpha}^{S}$

(c) if $\alpha=\beta+1, \beta \notin S$ then $M_{\beta}^{S}<_{\lambda^{+}}^{+} M_{\alpha}^{S}$

(d) if $\alpha=\beta+1, \beta \in S$ then $\left(M_{\beta}^{S}, M_{\alpha}^{S}\right) \cong(M, N)$

(e) if $\beta<\alpha, \beta \notin S$ then $M_{\beta}^{S} \leq_{\lambda^{+}}^{*} M_{\alpha}^{S}$.

We use freely the transitivity $(9.4(4))$ and continuity (9.6) of $\leq_{\lambda}^{*}$ and $\left[M^{a} \leq_{\mathfrak{K}}\right.$ $M^{b} \leq_{\mathfrak{K}} M^{c}$ in $K_{\lambda}, \neg\left(M^{a} \leq_{\lambda^{+}}^{*} M^{b} \Rightarrow \neg\left(M^{a} \leq_{\lambda^{+}}^{*} M^{c}\right)\right] 9.4(3)$.

For $\alpha=0$ no problem.

For $\alpha$ limit no problem.

For $\alpha=\beta+1, \beta \notin S$ no problem.

For $\alpha=\beta+1, \beta \in S$ so $\operatorname{cf}(\beta)=\lambda^{+}$, let $\left\langle\gamma_{i}: i\left\langle\lambda^{+}\right\rangle\right.$be increasing continuous with limit $\beta$ and $\operatorname{cf}\left(\gamma_{i}\right) \leq \lambda^{+}$, hence $\gamma_{i} \notin S$. By 9.3(2) without loss of generality $M_{\gamma_{i}}<_{\lambda^{+}}^{*} M_{\gamma_{i+1}}$. Now use 10.3 (and the uniqueness $(9.5(2))$ ). $\quad \square_{10.4}$ 
10.5 Conclusion. Assume $I\left(\lambda^{+2}, K\right)<2^{\lambda^{+2}}$ (in addition to hypothesis 10.1).

1) $\leq_{\lambda^{+}}^{*}=\leq_{\mathfrak{K}} \mid K_{\lambda^{+}}$.

2) $\left(K_{\lambda^{+}}, \leq_{\mathfrak{K}}\right)$, has disjoint amalgamation so no $M \in K_{\lambda^{+2}}$ is $\leq_{\mathfrak{K}^{-}}$maximal.

3) $K_{\lambda+3} \neq \emptyset$.

Proof. 1) By 10.4.

2) By 9.11 (and part (1)).

3) By 10.5(2) and 2.6(8) with $\lambda$ there replaced by $\lambda^{+}$here.

So we have finally proved the main theorem. Though not directly contributing to our main theme, we remark some more consequences of $\leq_{\mathfrak{K}} \mid K_{\lambda^{+}} \neq \leq_{\lambda^{+}}^{*}$.

10.6 Claim. $(*)_{0} \Leftrightarrow(*)_{1}$ where

$(*)_{0}$ for some $M \leq_{\mathfrak{K}} N$ from $K_{\lambda^{+}}$, we do not have $M \leq_{\lambda^{+}}^{*} N$

$(*)_{1}$ for some $M \leq_{\mathfrak{K}} N$ from $K_{\lambda^{+}}$we have:

if $a \in N \backslash M$ then $\operatorname{tp}(a, M, N)$ is not realized in any $M$ such that $M^{*} \leq_{\lambda^{+}}^{*} M \in K_{\lambda^{+}}$

10.7 Definition. Assume $M_{0}<_{\mathfrak{K}} M_{1}$ are in $K_{\lambda^{+}}$, and $\bar{M}^{\ell}=\left\langle M_{\ell, i}: i<\lambda^{+}\right\rangle$is a $\leq_{\mathfrak{K}}$-representation of $M_{\ell}$ for $\ell=0,1$. Let

$$
\begin{aligned}
\mathscr{S}_{0}\left(\bar{M}^{0}, \bar{M}^{1}\right)=\left\{\delta<\lambda^{+}:\right. & M_{1, \delta} \cap M_{0}=M_{0, \delta} \\
& \text { and not } \left.N F_{\lambda}\left(M_{0, \delta}, M_{0, \delta+1}, M_{1, \delta}, M_{1}\right)\right\}
\end{aligned}
$$

$$
\mathscr{S}_{1}\left(M_{0}, M_{1}\right)=\mathscr{S}_{0}\left(\bar{M}^{0}, \bar{M}^{1}\right) / D_{\lambda^{+}} \text {(well defined). }
$$

(c) $\quad J$ is the normal ideal on $\lambda^{++}$generated by sets of the form $\mathscr{S}_{0}\left(M^{0}, M^{1}\right)$ where $M^{0}, M^{1}$ are as above.

10.8Comment: An earlier try for 10.4 was:

1) For every $S \in J$ for some $\bar{M}^{0}, M^{1}$ as in 10.7 we have $S=S_{0}\left(\bar{M}^{0}, \bar{M}^{1}\right)$.

2) If $S_{1}=S_{1}\left(M^{0}, M^{1}\right)$ is stationary then for some $\bar{M}=\left\langle M_{i}: i<\lambda^{+}\right\rangle$a representation of $M=\bigcup_{i<\lambda^{+}} M_{i} \in K_{\lambda^{+}}$for every $S \subseteq S_{1}$ for some $\bar{M}^{\prime}$, we have

$\bar{M}^{1}=\left\langle M_{i}^{1}: i<\lambda^{+}\right\rangle, M_{1}<_{\mathfrak{K}} M^{1}=\bigcup_{i<\lambda^{+}} M_{i}^{1}, M_{i}^{1} \cap M=M_{i}$ and

$S_{0}\left(\bar{M}, \bar{M}^{1}\right)=S \bmod \mathscr{D}_{\lambda^{+}}$. 
3) If $\leq_{\lambda^{+}}^{*} \neq \leq_{\mathfrak{K}} \mid K_{\lambda^{+}}$and $I\left(\lambda^{+2}, K\right)<2^{\lambda^{+2}}$ then for some stationary $S \subseteq \lambda^{+}$we have:

(a) $\mathscr{D}_{\lambda^{+}} \uparrow S$ is $\lambda^{++}$-saturated

(b) $\bar{M}^{0}, \bar{M}^{1}$ as in 10.4 implies $S_{1}\left(\bar{M}^{0}, \bar{M}^{1}\right) \subseteq S \bmod D_{\lambda^{+}}$.

4) If $\leq_{\lambda^{+}}^{*} \neq \leq_{\mathfrak{K}} \mid K_{\lambda^{+}}$and $2^{\lambda}<2^{\lambda^{+}}<2^{\lambda^{+2}}$ then $I\left(\lambda^{+2}, K\right)=2^{\lambda^{+2}}$.

Proof. 1) First we prove only " $S \subseteq S_{0}\left(\bar{M}^{0}, \bar{M}^{1}\right)$ ". Easy as $\mathfrak{K}_{\lambda^{+}}$has amalgamation and

$\bigotimes$ if $M_{0} \leq_{\mathfrak{K}} M_{1} \leq_{\mathfrak{K}} M_{2}$ are in $K_{\lambda^{+}}, \bar{M}^{\ell}$

representing the $S_{0}\left(\bar{M}^{0}, \bar{M}^{1}\right) \subseteq S_{0}\left(\bar{M}^{0}, \bar{M}^{2}\right)$.

Now for equality use part (2).

2) Similar to the proof of 10.2 .

3) Suppose $S^{*}=S_{1}\left(\bar{M}^{0}, \bar{M}^{2}\right)$ is stationary, let $\bar{S}=\left\langle S_{\alpha}^{\prime}: \alpha<\lambda^{++}\right\rangle$be such that $S_{\alpha}^{\prime} \in J$. We can build a model $M^{\bar{S}} \in K_{\lambda^{+2}}$ and a representation $\left\langle M_{\alpha}^{\bar{S}}: \alpha<\lambda^{++}\right\rangle$ such that

$$
S_{1}\left(M_{\alpha}, M_{\alpha+1}\right)=S_{\alpha}^{\prime} / D_{\lambda^{++}} .
$$

4) By $\S 3$ (using the proof of part (2). 


\section{REFERENCES.}

[DvSh 65] Keith J. Devlin and Saharon Shelah. A weak version of $\diamond$ which follows from $2^{\aleph_{0}}<2^{\aleph_{1}}$. Israel Journal of Mathematics, 29:239-247, 1978.

[GrSh 266] Rami Grossberg and Saharon Shelah. ***. . Does not exist.

[Ha61] Andras Hajnal. Proof of a conjecture of S.Ruziewicz. Fundamenta Mathematicae, 50:123-128, 1961.

[HaSh 323] Bradd Hart and Saharon Shelah. Categoricity over $P$ for first order $T$ or categoricity for $\phi \in \mathrm{L}_{\omega_{1} \omega}$ can stop at $\aleph_{k}$ while holding for $\aleph_{0}, \cdots, \aleph_{k-1}$. Israel Journal of Mathematics, 70:219-235, 1990.

[KlSh 362] Oren Kolman and Saharon Shelah. Categoricity of Theories in $L_{\kappa, \omega}$, when $\kappa$ is a measurable cardinal. Part 1. Fundamenta Mathematicae, 151:209-240, 1996.

[MaSh 285] Michael Makkai and Saharon Shelah. Categoricity of theories in $L_{\kappa \omega}$, with $\kappa$ a compact cardinal. Annals of Pure and Applied Logic, 47:4197, 1990 .

[Mw85] Johann A. Makowsky. Compactnes, embeddings and definability. In J. Barwise and S. Feferman, editors, Model-Theoretic Logics, pages 645-716. Springer-Verlag, 1985.

[MkSh 313] Alan H. Mekler and Saharon Shelah. Diamond and $\lambda$-systems. Fundamenta Mathematicae, 131:45-51, 1988.

[Sh 600] Saharon Shelah. Categoricity in abstract elementary classes: going up inductive step. in preparation.

[Sh 394] Saharon Shelah. Categoricity of abstract classes with amalgamation. Annals of Pure and Applied Logic, accepted.

[Sh 472] Saharon Shelah. Categoricity of Theories in $L_{\kappa^{*} \omega}$, when $\kappa^{*}$ is a measurable cardinal. Part II. Fundamenta Mathematicae, submitted.

[Sh 638] Saharon Shelah. More on Weak Diamond. in preparation.

[Sh:e] Saharon Shelah. Non-structure theory, accepted. Oxford University Press.

[Sh 460] Saharon Shelah. The Generalized Continuum Hypothesis revisited. Israel Journal of Mathematics, accepted.

[Sh:b] Saharon Shelah. Proper forcing, volume 940 of Lecture Notes in Mathematics. Springer-Verlag, Berlin-New York, xxix+496 pp, 1982.

[Sh 87b] Saharon Shelah. Classification theory for nonelementary classes, I. The number of uncountable models of $\psi \in L_{\omega_{1}, \omega}$. Part B. Israel Journal of Mathematics, 46:241-273, 1983.

[Sh 87a] Saharon Shelah. Classification theory for nonelementary classes, I. The number of uncountable models of $\psi \in L_{\omega_{1}, \omega}$. Part A. Israel Journal of Mathematics, 46:212-240, 1983. 
[Sh 88] Saharon Shelah. Classification of nonelementary classes. II. Abstract elementary classes. In Classification theory (Chicago, IL, 1985), volume 1292 of Lecture Notes in Mathematics, pages 419-497. Springer, Berlin, 1987. Proceedings of the USA-Israel Conference on Classification Theory, Chicago, December 1985; ed. Baldwin, J.T.

[Sh 300] Saharon Shelah. Universal classes. In Classification theory (Chicago, IL, 1985), volume 1292 of Lecture Notes in Mathematics, pages 264418. Springer, Berlin, 1987. Proceedings of the USA-Israel Conference on Classification Theory, Chicago, December 1985; ed. Baldwin, J.T.

[Sh:c] Saharon Shelah. Classification theory and the number of nonisomorphic models, volume 92 of Studies in Logic and the Foundations of Mathematics. North-Holland Publishing Co., Amsterdam, xxxiv +705 pp, 1990.

[Sh 351] Saharon Shelah. Reflecting stationary sets and successors of singular cardinals. Archive for Mathematical Logic, 31:25-53, 1991.

[Sh:f] Saharon Shelah. Proper and improper forcing. Perspectives in Mathematical Logic. Springer, 1998. 